- Supporting Information -

\title{
Mechanism of the Palladium-Catalyzed Asymmetric Borylative Migration of Enol Perfluorosulfonates: Insights into an Enantiofacial-Selective Transmetalation
}

\section{Part II - Computational Details}

Helen A. Clement, ${ }^{1}$ Mohamad Estaitie, ${ }^{1}$ You-Ri Kim, ${ }^{1}$ Dennis G. Hall, ${ }^{* 1}$ Claude Y. Legault*2

(1) Department of Chemistry, University of Alberta Edmonton, Alberta, T6G 2G2, Canada dennis.hall@ualberta.ca

(2) Department of Chemistry, University of Sherbrooke 2500 boul. de l'Université Sherbrooke, Québec, J1K 2R1, Canada claude.legault@usherbrooke.ca 


\section{Table of Contents}

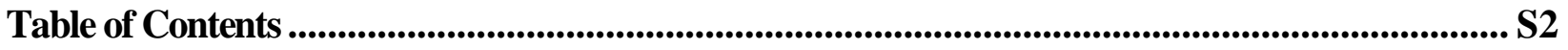

Computational Details............................................................................................................................ S3

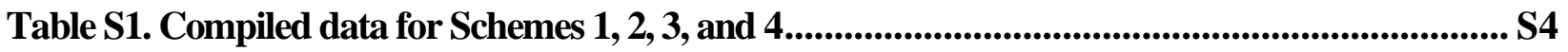

Table S2. Compiled data for Scheme 5 .................................................................................... S5

Scheme S1. Free energy diagram $(\mathrm{kcal} / \mathrm{mol})$ of path leading to Int-11 ......................................... S6

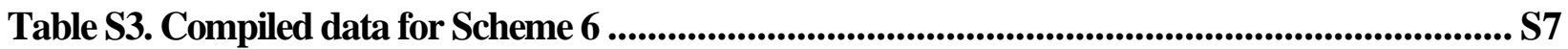

Figure S1. Optimized Structures and Analysis of TS-4 and TS-7 ....................................................... S8

Figure S2. Relaxed Scan of Pd-C Dihedral Angle of Int-5 ${ }^{\text {Tania }}$ Conformer..................................... S8

Cartesian Coordinates of Optimized Structures .......................................................................... S9

Cartesian Coordinates of Optimized Taniaphos Species ............................................................. S48

References ......................................................................................................................................... S82 


\section{Computational Details}

The geometry optimizations were performed using the Gaussian 16 software package ${ }^{1}$ with the $\mathrm{M}^{2} 6^{2}$ density functional, in combination with the 6-31G(d) ${ }^{3}$ basis set for all atoms except palladium (and iron for calculations involving Taniaphos ligand), for which LANL2DZ(ECP) was used. ${ }^{4}$ Unless otherwise stated, the default $(99,590)$ density grid was used for numerical integration in the calculations. The structures were optimized in the gas phase and include the Grimme dispersion correction using the original D3 damping function (D3(0)). ${ }^{5}$ Harmonic vibrational frequencies were computed for all optimized structures to verify that they were either minima or transition states, possessing zero or one imaginary frequency, respectively. Single points energy calculations were performed using the M06 density functional, with the $6-31+G(d, p)$ basis set for all atoms except palladium (and iron for calculations involving Taniaphos ligand), for which $\mathrm{SDD}(\mathrm{MWB} 28)^{6}$ was used. The single points were also performed using a solvation model (SMD) for diethyl ether, ${ }^{7}$ and include the Grimme dispersion correction using the original D3 damping

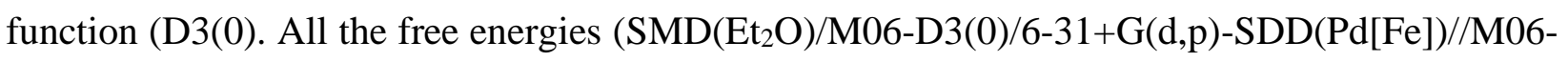
$\mathrm{D} 3(0) / 6-31 \mathrm{G}(\mathrm{d}) \mathrm{LANL} 2 \mathrm{DZ}\left(\mathrm{Pd}[\mathrm{Fe})\right.$ ) are reported in $\mathrm{kcal} \mathrm{mol}^{-1}$. They incorporate unscaled thermochemical corrections based on the vibrational analyses, using a temperature of $298.15 \mathrm{~K}$ and pressure of $1 \mathrm{~atm}$, using the correction to vibrational entropy reported by Grimme. ${ }^{8}$ Additionally, the free energies were corrected to a $1 \mathrm{M}$ standard state at $298.15 \mathrm{~K}$ using a $+1.89 \mathrm{kcal}$

$\mathrm{mol}^{-1}$ correction. For the calculations in relation to the stereoinduction process, involving the Taniaphos Ligand, Boltzmann averages were reported, as numerous energetically similar conformers were found (see Table S3). 


\section{Table S1. Compiled data for Schemes 1, 2, 3, and 4}

\begin{tabular}{|c|c|c|c|c|c|}
\hline Computed Species & SCF(M1) & SCF(M2) & Gcorr(M1) & $\mathrm{G}_{298(1 \mathrm{M})}$ & $\Delta \mathrm{G}_{298}$ \\
\hline Cat & $-2,272.22742$ & $-2,273.50994$ & 0.47081 & $-2,273.03611$ & \\
\hline PhNHMe2_cation & -366.28643 & -366.38967 & 0.15462 & -366.23203 & \\
\hline $\mathrm{PhNMe} 2$ & -365.91783 & -365.95519 & 0.14020 & -365.81197 & \\
\hline pinBH & -411.59665 & -411.63358 & 0.15838 & -411.47218 & \\
\hline TfO_anion & -961.27786 & -961.38882 & -0.00318 & -961.38898 & \\
\hline $1 \mathrm{a}$ & $-1,230.92814$ & $-1,230.98548$ & 0.10401 & $-1,230.87846$ & \\
\hline PhNHMe2_TfO_ion_pair & $-1,327.73515$ & $-1,327.82091$ & 0.17519 & $-1,327.64270$ & \\
\hline Int-1 & $-3,503.20933$ & $-3,504.54597$ & 0.60379 & $-3,503.93917$ & -15.4 \\
\hline TS-1 & $-3,503.17683$ & $-3,504.52211$ & 0.59982 & $-3,503.91927$ & -2.9 \\
\hline Int-2 & $-3,503.21417$ & $-3,504.56001$ & 0.60267 & $-3,503.95432$ & -24.9 \\
\hline Int-3 & $-2,907.74594$ & $-2,909.11287$ & 0.74783 & $-2,908.36202$ & $-24.9(-15.4)$ \\
\hline Int-4 & $-2,541.78276$ & $-2,543.12311$ & 0.57844 & $-2,542.54165$ & -19.7 \\
\hline Int-5 & $-2,953.41865$ & $-2,954.78528$ & 0.76356 & $-2,954.01870$ & -22.7 \\
\hline TS-2 & $-2,953.40623$ & $-2,954.77760$ & 0.76248 & $-2,954.01210$ & -18.6 \\
\hline Int-6 & $-2,953.44946$ & $-2,954.81932$ & 0.76474 & $-2,954.05157$ & -43.4 \\
\hline TS-3 & $-3,319.34580$ & $-3,320.74639$ & 0.92656 & $-3,319.81682$ & -14.0 \\
\hline TS-4 & $-3,319.38433$ & $-3,320.77597$ & 0.92869 & $-3,319.84426$ & -31.3 \\
\hline Int-10 & $-2,953.05950$ & $-2,954.39517$ & 0.75459 & $-2,953.63756$ & -60.8 \\
\hline TS-5 & $-2,953.44638$ & $-2,954.81760$ & 0.76270 & $-2,954.05188$ & -43.5 \\
\hline Int-7 & $-2,953.45680$ & $-2,954.82706$ & 0.76606 & $-2,954.05798$ & -47.4 \\
\hline Int-7' & $-2,953.44936$ & $-2,954.81699$ & 0.76654 & $-2,954.04743$ & -40.8 \\
\hline TS-6 & $-2,953.43951$ & $-2,954.80964$ & 0.76280 & $-2,954.04382$ & -38.5 \\
\hline Int-8 & $-2,953.44612$ & $-2,954.81457$ & 0.76327 & $-2,954.04828$ & -41.3 \\
\hline TS-7 & $-3,319.38760$ & $-3,320.78391$ & 0.92705 & $-3,319.85385$ & -37.3 \\
\hline Int-9 & $-2,953.04098$ & $-2,954.37799$ & 0.75282 & $-2,953.62215$ & -51.1 \\
\hline Product_3 & -680.77000 & -680.82861 & 0.25486 & -680.57074 & -41.5 \\
\hline Product_5 & -680.77079 & -680.82967 & 0.25513 & -680.57152 & -42.0 \\
\hline
\end{tabular}

Values in blue are relative to Int-3 being set to $-24.9 \mathrm{kcal} / \mathrm{mol}$

SCF(M1): Geometry optimization with M06-D3/6-31G(d) + LANL2DZ(Pd) theory model in the gas phase (M1).

SCF(M2): Single point energy with M06-D3/6-31+G(d,p) + SDD(Pd) and SMD(Et $\left.{ }_{2} \mathrm{O}\right)$ solvation model (M2).

Gcorr(M1): Free energy correction obtained from frequency calculation of converged geometries with M1.

G298(1M): Free energy M2//M1 obtained from SCF(M2) + $\mathbf{G}_{\text {corr }}(\mathbf{M 1})+\mathbf{1 . 8 9} \mathrm{kcal} / \mathrm{mol}$ (1 atm to $1 \mathrm{M}$ corr.)

$\Delta \mathbf{G}_{298}$ : Relative free energies with respect to the reference molecules (defined as $\Delta \mathrm{G}_{298}=0$ ). 


\section{Table S2. Compiled data for Scheme 5}

\begin{tabular}{|c|c|c|c|c|c|}
\hline Computed Species & SCF(M1) & SCF(M2) & Gcorr(M1) & $\mathrm{G}_{298(1 \mathrm{M})}$ & $\Delta \mathrm{G}_{298}$ \\
\hline Cat & $-2,272.22742$ & $-2,273.50994$ & 0.47081 & $-2,273.03611$ & \\
\hline PhNHMe2_cation & -366.28643 & -366.38967 & 0.15462 & -366.23203 & \\
\hline PhNMe2 & -365.91783 & -365.95519 & 0.14020 & -365.81197 & \\
\hline pinBH & -411.59665 & -411.63358 & 0.15838 & -411.47218 & \\
\hline TfO_anion & -961.27786 & -961.38882 & -0.00318 & -961.38898 & \\
\hline Cyclohexenyl_Triflate & $-1,195.03822$ & $-1,195.09400$ & 0.12834 & $-1,194.96265$ & \\
\hline PhNHMe2_TfO_ion_pair & $-1,327.73515$ & $-1,327.82091$ & 0.17519 & $-1,327.64270$ & \\
\hline Int-S1 & $-3,467.31780$ & $-3,468.65285$ & 0.62818 & $-3,468.02166$ & -14.4 \\
\hline TS-S1 & $-3,467.28474$ & $-3,468.62924$ & 0.62373 & $-3,468.00249$ & -2.3 \\
\hline Int-S2 & $-3,467.32006$ & $-3,468.66363$ & 0.62514 & $-3,468.03547$ & -23.0 \\
\hline Int-S3 & $-2,871.85369$ & $-2,873.21858$ & 0.77096 & $-2,872.44460$ & $-23.0(-14.3)$ \\
\hline Int-S4 & $-2,505.89187$ & $-2,507.22920$ & 0.60229 & $-2,506.62389$ & -17.6 \\
\hline Int-S5 & $-2,917.52409$ & $-2,918.88965$ & 0.78616 & $-2,918.10047$ & -20.3 \\
\hline TS-S2 & $-2,917.51778$ & $-2,918.88601$ & 0.78715 & $-2,918.09584$ & -17.4 \\
\hline Int-11 & $-2,917.55876$ & $-2,918.92560$ & 0.78836 & $-2,918.13422$ & -41.5 \\
\hline TS-8 & $-3,283.48876$ & $-3,284.88068$ & 0.95195 & $-3,283.92571$ & -28.6 \\
\hline Int-15 & $-2,917.16543$ & $-2,918.49869$ & 0.77821 & $-2,917.71746$ & -57.2 \\
\hline TS-9 & $-2,917.55612$ & $-2,918.92365$ & 0.78631 & $-2,918.13432$ & -41.6 \\
\hline Int-12 & $-2,917.56601$ & $-2,918.93345$ & 0.79053 & $-2,918.13990$ & -45.1 \\
\hline Int-12' & $-2,917.55843$ & $-2,918.92557$ & 0.78962 & $-2,918.13293$ & -40.7 \\
\hline TS-10 & $-2,917.53971$ & $-2,918.91026$ & 0.78651 & $-2,918.12073$ & -33.0 \\
\hline Int-13 & $-2,917.54237$ & $-2,918.91242$ & 0.78725 & $-2,918.12214$ & -33.9 \\
\hline TS-11 & $-3,283.48186$ & $-3,284.87985$ & 0.95114 & $-3,283.92569$ & -28.6 \\
\hline Int-14 & $-2,917.14307$ & $-2,918.48076$ & 0.77562 & $-2,917.70212$ & -47.5 \\
\hline Product_12 & -644.86852 & -644.92707 & 0.27810 & -644.64595 & -35.0 \\
\hline Product_13 & -644.87776 & -644.93467 & 0.27880 & -644.65285 & -39.3 \\
\hline
\end{tabular}

Values in blue are relative to Int-S3 being set to $-23.0 \mathrm{kcal} / \mathrm{mol}$

SCF(M1): Geometry optimization with M06-D3/6-31G(d) + LANL2DZ(Pd) theory model in the gas phase (M1).

SCF(M2): Single point energy with M06-D3/6-31+G(d,p) + SDD(Pd) and $\mathrm{SMD}\left(\mathrm{Et}_{2} \mathrm{O}\right)$ solvation model (M2).

Gcorr(M1): Free energy correction obtained from frequency calculation of converged geometries with M1.

$\mathbf{G}_{298(1 M)}$ : Free energy M2//M1 obtained from SCF(M2) + $\mathbf{G}_{\text {corr }}(\mathbf{M 1})+\mathbf{1 . 8 9} \mathrm{kcal} / \mathrm{mol}$ (1 atm to $1 \mathrm{M}$ corr.)

$\Delta \mathbf{G}_{298}$ : Relative free energies with respect to the reference molecules (defined as $\Delta \mathrm{G}_{298}=0$ ). 


\section{Scheme S1. Free energy diagram (kcal/mol) of path leading to Int-11}

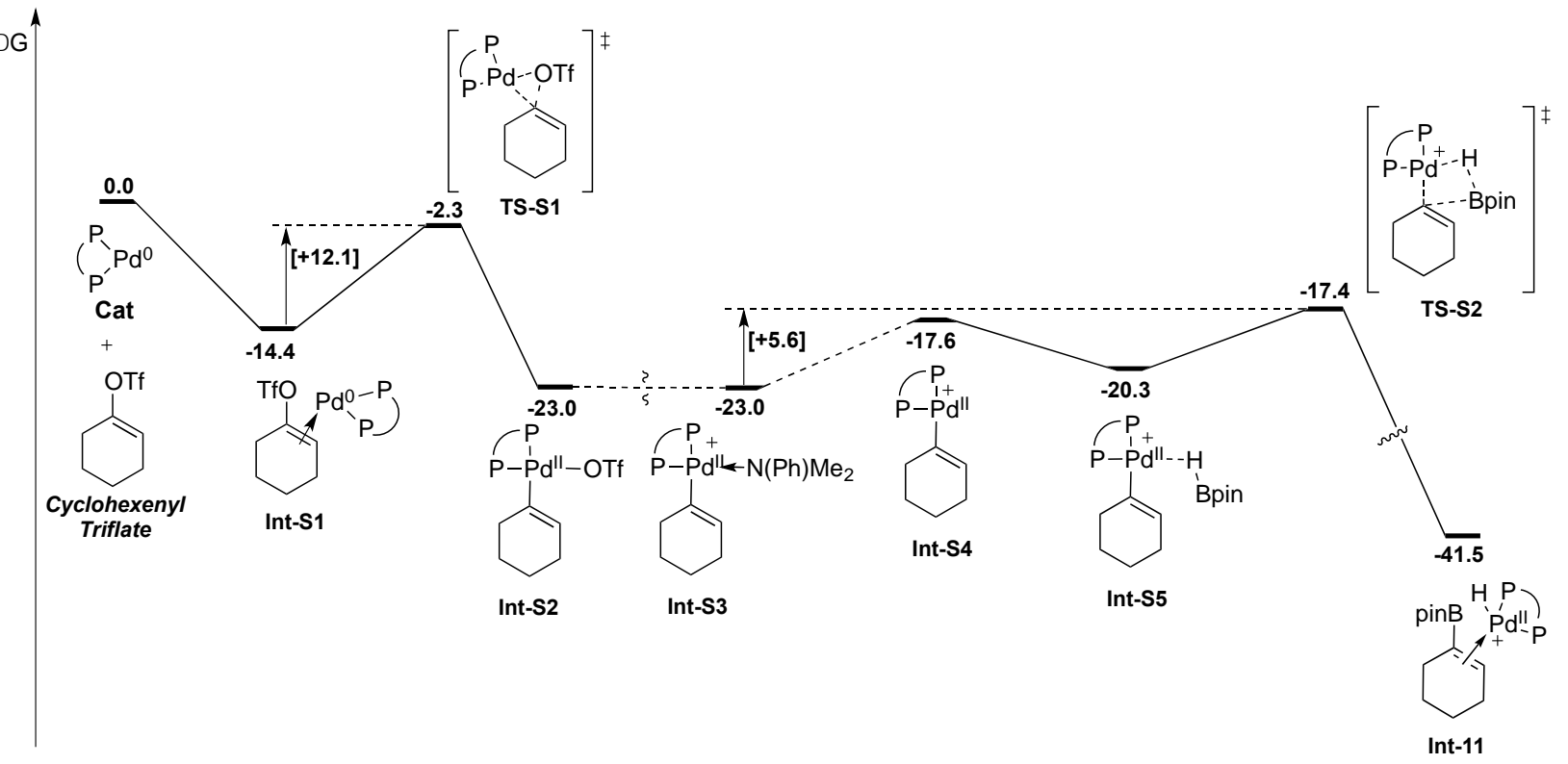


Table S3. Compiled data for Scheme 6

\begin{tabular}{|c|c|c|c|c|c|c|c|c|}
\hline Computed Species & SCF(M1) & SCF(M2) & Gcorr & $\mathrm{G}_{298(1 \mathrm{M})}$ & $\Delta \mathrm{G}_{1}$ & Population & $\Delta \mathrm{G}_{2}$ & $\Delta \mathrm{G}_{3}$ \\
\hline Int-4RTania_conf1 & $-2,918.23416$ & $-2,920.19530$ & 0.75161 & $-2,919.44067$ & 7.0 & $63.8 \%$ & 4.5 & \\
\hline Int-4RTania_conf2 & $-2,918.23457$ & $-2,920.19543$ & 0.75227 & $-2,919.44014$ & 7.3 & $36.2 \%$ & 2.6 & \\
\hline Int $-4 R^{\text {Tania }}$ & & & & & & $100 \%$ & 7.1 & 0.1 \\
\hline Int-4STania_conf1 & $-2,918.23423$ & $-2,920.19603$ & 0.75207 & $-2,919.44094$ & 6.8 & $36.5 \%$ & 2.5 & \\
\hline Int-4STania_conf2 & $-2,918.23291$ & $-2,920.19656$ & 0.75271 & $-2,919.44083$ & 6.9 & $32.4 \%$ & 2.2 & \\
\hline Int-4STania_conf3 & $-2,918.23152$ & $-2,920.19557$ & 0.75215 & $-2,919.44041$ & 7.1 & $20.7 \%$ & 1.5 & \\
\hline Int-4STania_conf4 & $-2,918.23242$ & $-2,920.19499$ & 0.75275 & $-2,919.43923$ & 7.9 & $5.9 \%$ & 0.5 & \\
\hline Int-4STania_conf5 & $-2,918.23230$ & $-2,920.19437$ & 0.75240 & $-2,919.43896$ & 8.1 & $4.5 \%$ & 0.4 & \\
\hline Int $-4 s^{\text {Tania }}$ & & & & & & $100 \%$ & 7.0 & 0.0 \\
\hline pinBH & -411.59665 & -411.63358 & 0.15838 & -411.47218 & & & & \\
\hline Int-5RTania_conf1 & $-3,329.87369$ & $-3,331.86100$ & 0.93723 & $-3,330.92076$ & 2.0 & $75.7 \%$ & 1.5 & \\
\hline Int-5RTania_conf2 & $-3,329.87169$ & $-3,331.85943$ & 0.93762 & $-3,330.91879$ & 3.3 & $9.4 \%$ & 0.3 & \\
\hline Int-5RTania_conf3 & $-3,329.87389$ & $-3,331.85866$ & 0.93734 & $-3,330.91830$ & 3.6 & $5.6 \%$ & 0.2 & \\
\hline Int-5RTania_conf4 & $-3,329.87302$ & $-3,331.85815$ & 0.93712 & $-3,330.91801$ & 3.7 & $4.1 \%$ & 0.2 & \\
\hline Int-5RTania_conf5 & $-3,329.87197$ & $-3,331.85898$ & 0.93832 & $-3,330.91765$ & 4.0 & $2.8 \%$ & 0.1 & \\
\hline Int-5RTania_conf6 & $-3,329.87212$ & $-3,331.85870$ & 0.93850 & $-3,330.91718$ & 4.3 & $1.7 \%$ & 0.1 & \\
\hline Int-5RTania_conf7 & $-3,329.87146$ & $-3,331.85639$ & 0.93699 & $-3,330.91638$ & 4.8 & $0.7 \%$ & 0.0 & \\
\hline Int $-5 R^{\text {Tania }}$ & & & & & & $100 \%$ & 2.4 & -4.6 \\
\hline Int-5STania_conf1 & $-3,329.87709$ & $-3,331.86485$ & 0.93786 & $-3,330.92397$ & 0.0 & $34.5 \%$ & 0.0 & \\
\hline Int-5STania_conf2 & $-3,329.87752$ & $-3,331.86403$ & 0.93714 & $-3,330.92388$ & 0.1 & $31.2 \%$ & 0.0 & \\
\hline Int-5STania_conf3 & $-3,329.87673$ & $-3,331.86336$ & 0.93703 & $-3,330.92331$ & 0.4 & $17.1 \%$ & 0.1 & \\
\hline Int-5STania_conf4 & $-3,329.87766$ & $-3,331.86256$ & 0.93696 & $-3,330.92258$ & 0.9 & $7.9 \%$ & 0.1 & \\
\hline Int-5STania_conf5 & $-3,329.87439$ & $-3,331.86085$ & 0.93583 & $-3,330.92200$ & 1.2 & $4.3 \%$ & 0.1 & \\
\hline Int-5STania_conf6 & $-3,329.87518$ & $-3,331.86292$ & 0.93814 & $-3,330.92176$ & 1.4 & $3.3 \%$ & 0.0 & \\
\hline Int-5STania_conf7 & $-3,329.87687$ & $-3,331.86317$ & 0.93905 & $-3,330.92111$ & 1.8 & $1.7 \%$ & 0.0 & \\
\hline Int-5STania_conf8 & $-3,329.87519$ & $-3,331.86006$ & 0.93783 & $-3,330.91921$ & 3.0 & $0.2 \%$ & 0.0 & \\
\hline Int $-5 s^{\text {Tania }}$ & & & & & & $100 \%$ & 0.3 & -6.7 \\
\hline TS-2RTania_conf1 & $-3,329.86250$ & $-3,331.85231$ & 0.93785 & $-3,330.91144$ & 7.9 & $56.5 \%$ & 4.4 & \\
\hline TS-2RTania_conf2 & $-3,329.86127$ & $-3,331.85021$ & 0.93641 & $-3,330.91078$ & 8.3 & $28.2 \%$ & 2.3 & \\
\hline TS-2RTania_conf3 & $-3,329.86292$ & $-3,331.84973$ & 0.93671 & $-3,330.91000$ & 8.8 & $12.4 \%$ & 1.1 & \\
\hline TS-2RTania_conf4 & $-3,329.86176$ & $-3,331.84854$ & 0.93689 & $-3,330.90863$ & 9.6 & $2.9 \%$ & 0.3 & \\
\hline TS- $2 R^{\text {Tania }}$ & & & & & & $100 \%$ & 8.1 & 1.1 \\
\hline TS-2STania_conf1 & $-3,329.86609$ & $-3,331.85546$ & 0.93706 & $-3,330.91538$ & 5.4 & $44.3 \%$ & 2.4 & \\
\hline TS-2STania_conf2 & $-3,329.86741$ & $-3,331.85607$ & 0.93798 & $-3,330.91507$ & 5.6 & $31.9 \%$ & 1.8 & \\
\hline TS-2STania_conf3 & $-3,329.86428$ & $-3,331.85382$ & 0.93714 & $-3,330.91367$ & 6.5 & $7.2 \%$ & 0.5 & \\
\hline TS-2STania_conf4 & $-3,329.86611$ & $-3,331.85459$ & 0.93798 & $-3,330.91360$ & 6.5 & $6.7 \%$ & 0.4 & \\
\hline TS-2STania_conf5 & $-3,329.86551$ & $-3,331.85390$ & 0.93760 & $-3,330.91328$ & 6.7 & $4.8 \%$ & 0.3 & \\
\hline TS-2STania_conf6 & $-3,329.86348$ & $-3,331.85190$ & 0.93610 & $-3,330.91278$ & 7.0 & $2.8 \%$ & 0.2 & \\
\hline TS-2STania_conf7 & $-3,329.86729$ & $-3,331.85244$ & 0.93742 & $-3,330.91201$ & 7.5 & $1.2 \%$ & 0.1 & \\
\hline TS-2STania_conf8 & $-3,329.86782$ & $-3,331.85322$ & 0.93831 & $-3,330.91189$ & 7.6 & $1.1 \%$ & 0.1 & \\
\hline TS- $2 s^{\text {Tania }}$ & & & & & & $100 \%$ & 5.8 & -1.3 \\
\hline
\end{tabular}

SCF(M1): Geometry optimization with M06-D3/6-31G(d) + LANL2DZ(Pd, Fe) theory model in the gas phase (M1).

SCF(M2): Single point energy with M06-D3/6-31+G(d,p) + SDD(Pd, Fe) and $\mathrm{SMD}\left(\mathrm{Et}_{2} \mathrm{O}\right)$ solvation model (M2).

$\mathbf{G}_{\text {corr }}(\mathbf{M 1})$ : Free energy correction obtained from frequency calculation of converged geometries with M1.

G298(1M): Free energy M2//M1 obtained from SCF(M2) + $\mathbf{G}$ corr $+1.89 \mathrm{kcal} / \mathrm{mol}$ (1 atm to $1 \mathrm{M}$ corr.)

$\Delta \mathbf{G}_{1}$ : Relative free energies (kcal/mol) with respect to the lowest conformer (Int-5STania_conf1, displayed in green).

$\Delta \mathbf{G}_{2}$ : Relative free energies ( $\mathrm{kcal} / \mathrm{mol}$ ) derived from Boltzmann averages (in bold) with respect to the lowest conformer.

$\Delta \mathbf{G}_{3}$ : Relative free energies $(\mathrm{kcal} / \mathrm{mol})$ derived from Boltzmann averages with respect to Int- $\mathbf{s}^{\text {Tania }}$ (as in Scheme 6). 
Figure S1. Optimized Structures and Analysis of TS-4 and TS-7

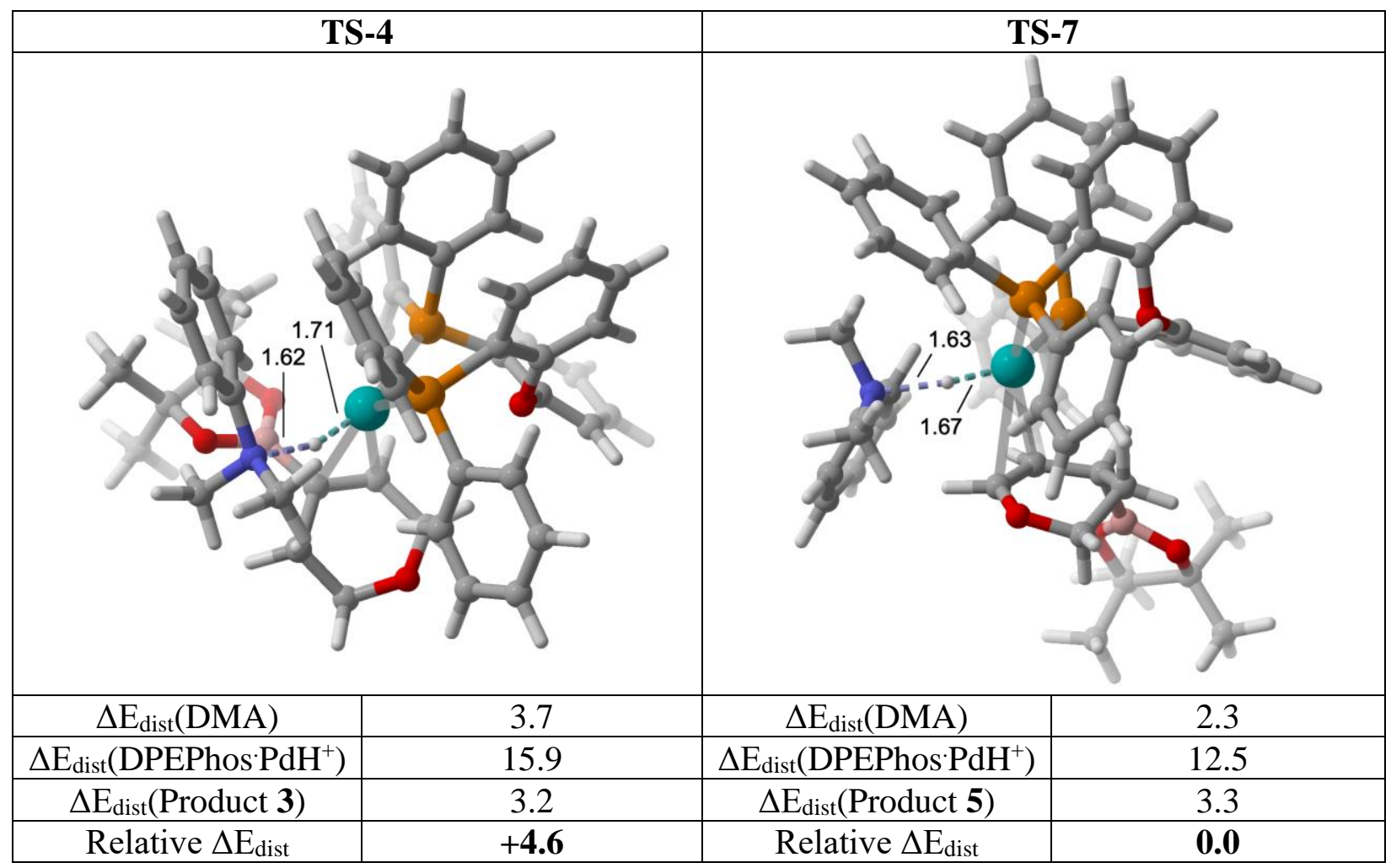

Computed distortion energies (in $\mathrm{kcal} / \mathrm{mol}$, using SCF(M1)) of the components of each computed TS.

Figure S2. Relaxed Scan of Pd-C Dihedral Angle of Int-5 $S^{\text {Tania }}$ Conformer
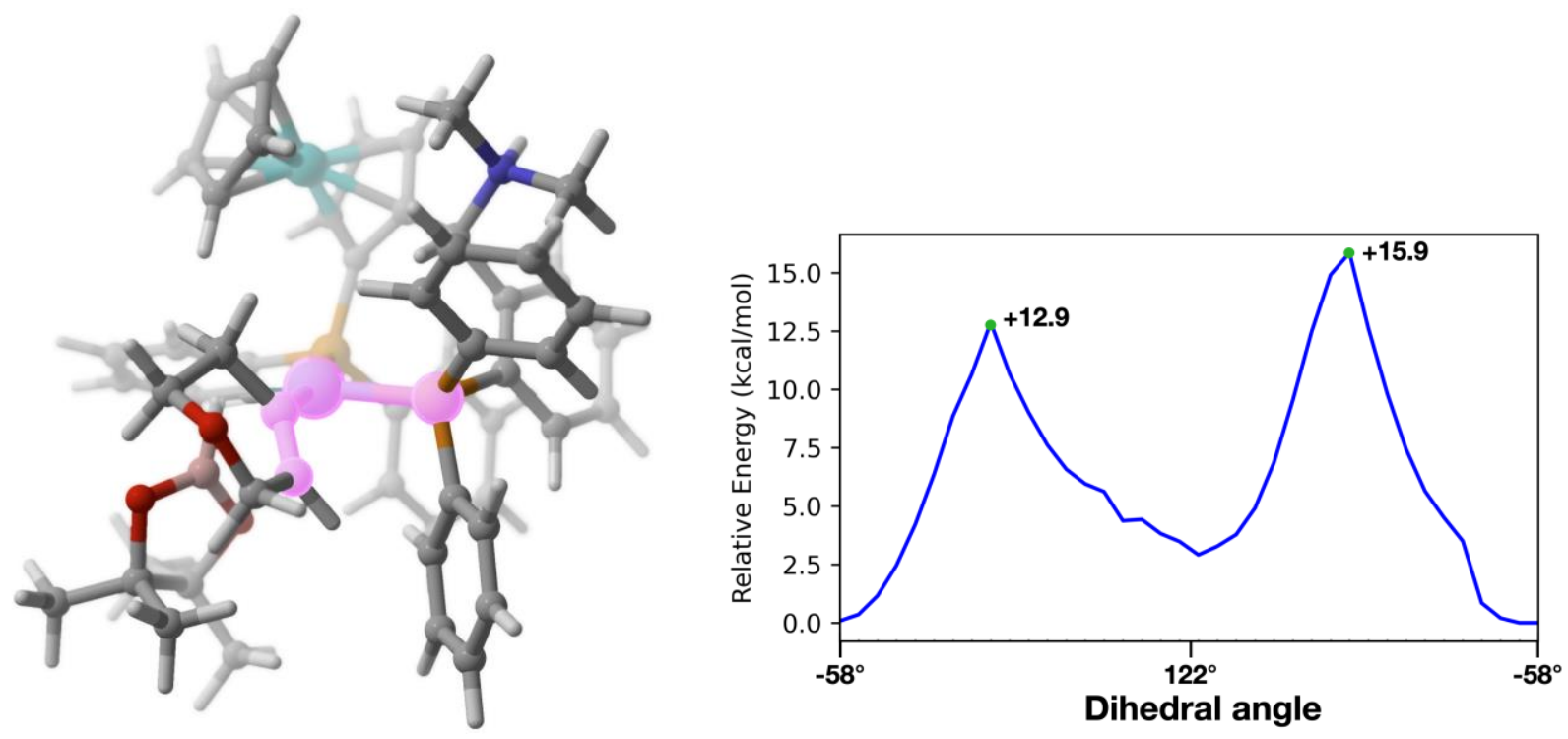


\section{Cartesian Coordinates of Optimized Structures}

\begin{tabular}{|c|c|c|c|}
\hline \multicolumn{4}{|l|}{ Cat } \\
\hline $\mathrm{Pd}$ & -1.372257 & -0.905726 & 1.033409 \\
\hline $\mathrm{P}$ & 0.769607 & -0.980320 & 1.938812 \\
\hline $\mathrm{P}$ & -1.968760 & 0.179892 & -0.931081 \\
\hline $\mathrm{C}$ & 1.240370 & 0.151558 & 3.304588 \\
\hline $\mathrm{C}$ & 1.854631 & 1.834874 & 5.449481 \\
\hline $\mathrm{C}$ & 2.437469 & 0.873093 & 3.316735 \\
\hline $\mathrm{C}$ & 0.350859 & 0.290577 & 4.372377 \\
\hline $\mathrm{C}$ & 0.658738 & 1.122720 & 5.443474 \\
\hline $\mathrm{C}$ & 2.742343 & 1.711033 & 4.384202 \\
\hline $\mathrm{H}$ & 3.132220 & 0.779411 & 2.480544 \\
\hline $\mathrm{H}$ & -0.595322 & -0.252675 & 4.349948 \\
\hline $\mathrm{H}$ & -0.042093 & 1.224031 & 6.270785 \\
\hline $\mathrm{H}$ & 3.676371 & 2.271378 & 4.383640 \\
\hline $\mathrm{H}$ & 2.092698 & 2.493609 & 6.283436 \\
\hline $\mathrm{C}$ & 1.799053 & -0.368410 & 0.533152 \\
\hline $\mathrm{C}$ & 3.045581 & 0.504033 & -1.825769 \\
\hline $\mathrm{C}$ & 1.405265 & 0.820628 & -0.104203 \\
\hline $\mathrm{C}$ & 2.848946 & -1.091973 & -0.035101 \\
\hline $\mathrm{C}$ & 3.471674 & -0.663544 & -1.205206 \\
\hline $\mathrm{C}$ & 2.012605 & 1.256289 & -1.274479 \\
\hline $\mathrm{H}$ & 3.173556 & -2.019253 & 0.436630 \\
\hline $\mathrm{H}$ & 4.282283 & -1.250099 & -1.633658 \\
\hline $\mathrm{H}$ & 1.667585 & 2.170002 & -1.755874 \\
\hline $\mathrm{H}$ & 3.512006 & 0.837133 & -2.751666 \\
\hline $\mathrm{C}$ & 1.642584 & -2.540289 & 2.363437 \\
\hline $\mathrm{C}$ & 2.907139 & -4.991122 & 2.821801 \\
\hline $\mathrm{C}$ & 1.168048 & -3.708464 & 1.758947 \\
\hline $\mathrm{C}$ & 2.751990 & -2.614531 & 3.209379 \\
\hline $\mathrm{C}$ & 3.377587 & -3.835911 & 3.439959 \\
\hline $\mathrm{C}$ & 1.801454 & -4.926479 & 1.978062 \\
\hline $\mathrm{H}$ & 0.288108 & -3.647923 & 1.113747 \\
\hline $\mathrm{H}$ & 3.128893 & -1.711954 & 3.691246 \\
\hline $\mathrm{H}$ & 4.238873 & -3.885258 & 4.104853 \\
\hline $\mathrm{H}$ & 1.425234 & -5.828943 & 1.498507 \\
\hline $\mathrm{H}$ & 3.399620 & -5.945323 & 3.003451 \\
\hline $\mathrm{C}$ & -0.900331 & -0.378974 & -2.325209 \\
\hline $\mathrm{C}$ & 0.775296 & -1.308412 & -4.363339 \\
\hline $\mathrm{C}$ & -0.633510 & 0.418319 & -3.444089 \\
\hline $\mathrm{C}$ & -0.310043 & -1.641828 & -2.238592 \\
\hline $\mathrm{C}$ & 0.521458 & -2.106395 & -3.252857 \\
\hline $\mathrm{C}$ & 0.196916 & -0.044686 & -4.458222 \\
\hline $\mathrm{H}$ & -1.068832 & 1.416693 & -3.514866 \\
\hline $\mathrm{H}$ & -0.493998 & -2.245996 & -1.346883 \\
\hline $\mathrm{H}$ & 0.983570 & -3.088582 & -3.166603 \\
\hline $\mathrm{H}$ & 0.399618 & 0.585837 & -5.322969 \\
\hline $\mathrm{H}$ & 1.432541 & -1.667163 & -5.154272 \\
\hline $\mathrm{C}$ & -1.526662 & 1.981335 & -0.876420 \\
\hline $\mathrm{C}$ & -0.831475 & 4.713605 & -0.736460 \\
\hline $\mathrm{C}$ & -0.378953 & 2.412924 & -0.190329 \\
\hline $\mathrm{C}$ & -2.309500 & 2.966614 & -1.489526 \\
\hline $\mathrm{C}$ & -1.970541 & 4.314467 & -1.426354 \\
\hline $\mathrm{C}$ & -0.036408 & 3.757898 & -0.115632 \\
\hline $\mathrm{H}$ & -3.210467 & 2.670231 & -2.026460 \\
\hline $\mathrm{H}$ & -2.603642 & 5.052640 & -1.915602 \\
\hline $\mathrm{H}$ & 0.856118 & 4.030051 & 0.445657 \\
\hline $\mathrm{H}$ & -0.561771 & 5.766472 & -0.676211 \\
\hline C & -3.650457 & 0.252876 & -1.675537 \\
\hline $\mathrm{C}$ & -6.287843 & 0.381794 & -2.605298 \\
\hline $\mathrm{C}$ & -4.703354 & 0.538219 & -0.797822 \\
\hline C & -3.938388 & 0.015733 & -3.021306 \\
\hline C & -5.251971 & 0.075552 & -3.480767 \\
\hline $\mathrm{C}$ & -6.010582 & 0.615704 & -1.259877 \\
\hline $\mathrm{H}$ & -4.485915 & 0.698681 & 0.260437 \\
\hline $\mathrm{H}$ & -3.133831 & -0.220458 & -3.717029 \\
\hline $\mathrm{H}$ & -5.463989 & -0.116619 & -4.531728 \\
\hline $\mathrm{H}$ & -6.818054 & 0.847251 & -0.566868 \\
\hline $\mathrm{H}$ & -7.313449 & 0.430421 & -2.968020 \\
\hline 0 & 0.403582 & 1.523729 & 0.511290 \\
\hline
\end{tabular}

SCF energy: -2272.227419360

M06-D3 (Gas Phase)/6-31G(d)+LANL2DZ (Pd) opt. No imaginary frequency

\begin{tabular}{rrr}
\multicolumn{4}{c}{$r$} \\
la
\end{tabular}

SCF energy: -1230.928137570

M06-D3 (Gas Phase)/6-31G(d)+LANL2DZ (Pd) opt. No imaginary frequency

\begin{tabular}{|c|c|c|c|}
\hline \multicolumn{4}{|c|}{ PhNMe2 } \\
\hline $\mathrm{N}$ & 1.321388 & 0.145453 & 0.000000 \\
\hline $\mathrm{C}$ & 2.035107 & -0.006175 & -1.243979 \\
\hline $\mathrm{H}$ & 1.774550 & 0.792192 & -1.954384 \\
\hline $\mathrm{H}$ & 3.110511 & 0.063195 & -1.054615 \\
\hline $\mathrm{C}$ & 2.035107 & -0.006175 & 1.243979 \\
\hline $\mathrm{H}$ & 3.110511 & 0.063195 & 1.054615 \\
\hline $\mathrm{H}$ & 1.774550 & 0.792192 & 1.954384 \\
\hline $\mathrm{C}$ & -0.062595 & 0.077108 & 0.000000 \\
\hline $\mathrm{C}$ & -2.890016 & -0.029362 & -0.000000 \\
\hline $\mathrm{C}$ & -0.790942 & 0.043196 & -1.204394 \\
\hline $\mathrm{C}$ & -0.790942 & 0.043196 & 1.204394 \\
\hline $\mathrm{C}$ & -2.178364 & -0.005666 & 1.194746 \\
\hline $\mathrm{C}$ & -2.178364 & -0.005666 & -1.194746 \\
\hline $\mathrm{H}$ & -0.272576 & 0.054716 & -2.159935 \\
\hline $\mathrm{H}$ & -0.272576 & 0.054716 & 2.159935 \\
\hline $\mathrm{H}$ & -2.709044 & -0.029321 & 2.146118 \\
\hline $\mathrm{H}$ & -2.709044 & -0.029321 & -2.146118 \\
\hline $\mathrm{H}$ & -3.977332 & -0.069910 & -0.000000 \\
\hline $\mathrm{H}$ & 1.835035 & -0.973833 & 1.736299 \\
\hline $\mathrm{H}$ & 1.835035 & -0.973833 & -1.736299 \\
\hline \multicolumn{4}{|c|}{$\begin{array}{l}\mathrm{SCF} \text { energy: }-365.917825740 \\
\end{array}$} \\
\hline \multicolumn{4}{|c|}{$\begin{array}{l}\text { SCF energy: }-365.917825740 \\
\text { M06-D3 (Gas Phase) /6-31G (d) +LANL2DZ (Pd) opt. } \\
\text { No imaginary freguency }\end{array}$} \\
\hline
\end{tabular}




\begin{tabular}{|c|c|c|c|}
\hline \multicolumn{4}{|c|}{ pinBH } \\
\hline O & -0.144247 & 0.011598 & 1.237798 \\
\hline B & 0.875670 & 0.518913 & 1.987389 \\
\hline 0 & 0.460357 & 1.280068 & 3.039906 \\
\hline $\mathrm{C}$ & -0.969931 & 1.100008 & 3.139709 \\
\hline $\mathrm{C}$ & -1.352847 & 0.658687 & 1.694635 \\
\hline $\mathrm{H}$ & 2.019274 & 0.316599 & 1.746155 \\
\hline $\mathrm{C}$ & -1.599997 & 1.830590 & 0.755567 \\
\hline $\mathrm{H}$ & -0.787284 & 2.566416 & 0.810225 \\
\hline $\mathrm{H}$ & -2.546799 & 2.338542 & 0.980327 \\
\hline $\mathrm{H}$ & -1.645372 & 1.454847 & -0.273750 \\
\hline $\mathrm{C}$ & -2.500583 & -0.325537 & 1.608748 \\
\hline $\mathrm{H}$ & -2.282055 & -1.254798 & 2.145117 \\
\hline $\mathrm{H}$ & -2.691348 & -0.579726 & 0.558816 \\
\hline $\mathrm{H}$ & -3.418989 & 0.112659 & 2.024246 \\
\hline $\mathrm{C}$ & -1.598521 & 2.398531 & 3.600358 \\
\hline $\mathrm{H}$ & -1.316477 & 3.238865 & 2.957433 \\
\hline $\mathrm{H}$ & -1.266314 & 2.628398 & 4.620230 \\
\hline $\mathrm{H}$ & -2.694559 & 2.316276 & 3.610805 \\
\hline $\mathrm{C}$ & -1.199872 & 0.012142 & 4.178913 \\
\hline $\mathrm{H}$ & -0.765611 & -0.944359 & 3.860438 \\
\hline $\mathrm{H}$ & -2.268563 & -0.139062 & 4.379094 \\
\hline $\mathrm{H}$ & -0.711632 & 0.308844 & 5.115043 \\
\hline
\end{tabular}

SCF energy: -411.596654053

M06-D3 (Gas Phase) /6-31G(d)+LANL2DZ (Pd) opt. No imaginary frequency

\begin{tabular}{|c|c|c|c|}
\hline \multicolumn{4}{|c|}{ PhNHMe2_cation } \\
\hline $\mathrm{N}$ & 2.373263 & 1.346046 & 0.924256 \\
\hline $\mathrm{C}$ & 3.090806 & 0.027485 & 0.937695 \\
\hline $\mathrm{H}$ & 2.698501 & -0.589634 & 0.125959 \\
\hline $\mathrm{H}$ & 4.162018 & 0.205891 & 0.809298 \\
\hline $\mathrm{C}$ & 2.880373 & 2.264065 & 1.998380 \\
\hline $\mathrm{H}$ & 3.953878 & 2.416444 & 1.856295 \\
\hline $\mathrm{H}$ & 2.339931 & 3.211067 & 1.929511 \\
\hline $\mathrm{C}$ & 0.896493 & 1.191680 & 0.956522 \\
\hline $\mathrm{C}$ & -1.840282 & 0.922642 & 0.978251 \\
\hline $\mathrm{C}$ & 0.171415 & 1.640983 & -0.136953 \\
\hline $\mathrm{C}$ & 0.293651 & 0.610248 & 2.064066 \\
\hline $\mathrm{C}$ & -1.090351 & 0.478956 & 2.064906 \\
\hline C & -1.213026 & 1.501924 & -0.119692 \\
\hline $\mathrm{H}$ & 0.667401 & 2.094731 & -0.996167 \\
\hline $\mathrm{H}$ & 0.873516 & 0.261148 & 2.917396 \\
\hline $\mathrm{H}$ & -1.583132 & 0.026481 & 2.921980 \\
\hline $\mathrm{H}$ & -1.797635 & 1.848453 & -0.968298 \\
\hline $\mathrm{H}$ & -2.922312 & 0.815359 & 0.988715 \\
\hline $\mathrm{H}$ & 2.694052 & 1.798460 & 2.969083 \\
\hline $\mathrm{H}$ & 2.905107 & -0.458574 & 1.898434 \\
\hline $\mathrm{H}$ & 2.590645 & 1.792771 & 0.025494 \\
\hline
\end{tabular}

SCF energy: -366.286429967

M0 6-D3 (Gas Phase) /6-31G(d)+LANL2DZ (Pd) opt. No imaginary frequency

$\begin{array}{cccc}\text { TfO_anion } & & & \\ - & & & \\ \text { O } & 1.623090 & -0.006154 & -1.860260 \\ \text { F } & 2.174640 & 2.836561 & -2.513735 \\ \text { S } & 0.463692 & 0.875341 & -2.055253 \\ \text { O } & 0.011414 & 1.033783 & -3.444341 \\ \text { C } & 1.168908 & 2.532625 & -1.685026 \\ \text { F } & 1.649278 & 2.595211 & -0.437735 \\ \text { O } & -0.600519 & 0.759472 & -1.048585 \\ \text { F } & 0.249708 & 3.497568 & -1.805380\end{array}$

SCF energy: -961.277856691

M06-D3 (Gas Phase)/6-31G(d)+LANL2DZ (Pd) opt. No imaginary frequency

\begin{tabular}{|c|c|c|c|}
\hline \multicolumn{4}{|c|}{ PhNHMe2_Tfo_ion_pair } \\
\hline $\mathrm{H}$ & -2.990788 & -0.096017 & 2.749804 \\
\hline $\mathrm{H}$ & -2.210495 & 1.456052 & 2.313386 \\
\hline $\mathrm{H}$ & -2.593722 & 1.083316 & 4.045442 \\
\hline $\mathrm{C}$ & -2.287941 & 0.675752 & 3.076512 \\
\hline $\mathrm{N}$ & -0.943917 & 0.043965 & 3.207326 \\
\hline $\mathrm{H}$ & 0.154679 & 1.760657 & 2.737477 \\
\hline $\mathrm{C}$ & 0.105572 & 1.039590 & 3.559244 \\
\hline $\mathrm{H}$ & -0.155996 & 1.531169 & 4.501466 \\
\hline $\mathrm{H}$ & 1.059146 & 0.514893 & 3.659083 \\
\hline S & -0.411121 & 0.348074 & -0.154481 \\
\hline 0 & -0.662138 & 1.599475 & 0.583319 \\
\hline 0 & -0.920923 & 0.237882 & -1.507863 \\
\hline 0 & -0.650173 & -0.849188 & 0.717467 \\
\hline $\mathrm{C}$ & 1.414911 & 0.321683 & -0.310709 \\
\hline $\mathrm{F}$ & 1.838985 & 1.362650 & -1.009575 \\
\hline $\mathrm{F}$ & 1.965767 & 0.378452 & 0.908909 \\
\hline $\mathrm{F}$ & 1.821701 & -0.792008 & -0.899775 \\
\hline $\mathrm{H}$ & -0.723906 & -0.303720 & 2.221694 \\
\hline $\mathrm{C}$ & -0.960574 & -1.136546 & 4.078321 \\
\hline $\mathrm{C}$ & -1.013868 & -3.391265 & 5.668047 \\
\hline C & -0.824985 & -2.383953 & 3.482648 \\
\hline $\mathrm{C}$ & -1.128238 & -0.992339 & 5.450290 \\
\hline $\mathrm{C}$ & -1.152708 & -2.131740 & 6.245764 \\
\hline $\mathrm{C}$ & -0.851692 & -3.515481 & 4.292332 \\
\hline $\mathrm{H}$ & -0.700826 & -2.459626 & 2. 402667 \\
\hline $\mathrm{H}$ & -1.238348 & -0.006247 & 5.900212 \\
\hline $\mathrm{H}$ & -1.280970 & -2.033723 & 7.321738 \\
\hline $\mathrm{H}$ & -0.744108 & -4.498884 & 3.839829 \\
\hline $\mathrm{H}$ & -1.033181 & -4.279944 & 6.295748 \\
\hline
\end{tabular}

SCF energy: -1327.735147640

M06-D3 (Gas Phase) /6-31G(d) +LANL2DZ (Pd) opt. No imaginary frequency 


\begin{tabular}{|c|c|c|c|}
\hline \multicolumn{4}{|c|}{ Int-1 } \\
\hline $\mathrm{Pd}$ & -1.505293 & -1.143153 & 1.144578 \\
\hline $\mathrm{P}$ & 0.820625 & -1.009688 & 1.723113 \\
\hline $\mathrm{P}$ & -1.829344 & 0.402029 & -0.671614 \\
\hline $\mathrm{C}$ & 1.334716 & 0.020525 & 3.144728 \\
\hline $\mathrm{C}$ & 2.036593 & 1.518871 & 5.393307 \\
\hline $\mathrm{C}$ & 2.592820 & 0.622812 & 3.224810 \\
\hline $\mathrm{C}$ & 0.431463 & 0.179539 & 4.197117 \\
\hline $\mathrm{C}$ & 0.781805 & 0.921539 & 5.320419 \\
\hline $\mathrm{C}$ & 2.940893 & 1.370269 & 4.344493 \\
\hline $\mathrm{H}$ & 3.301587 & 0.502432 & 2.404013 \\
\hline $\mathrm{H}$ & -0.556382 & -0.280028 & 4.122855 \\
\hline $\mathrm{H}$ & 0.070645 & 1.040462 & 6.136359 \\
\hline $\mathrm{H}$ & 3.922537 & 1.838687 & 4.399803 \\
\hline $\mathrm{H}$ & 2.310465 & 2.105654 & 6.268825 \\
\hline $\mathrm{C}$ & 1.843863 & -0.387893 & 0.329227 \\
\hline $\mathrm{C}$ & 2.974972 & 0.497908 & -2.076956 \\
\hline $\mathrm{C}$ & 1.514937 & 0.863677 & -0.211632 \\
\hline $\mathrm{C}$ & 2.786677 & -1.163829 & -0.346647 \\
\hline $\mathrm{C}$ & 3.351291 & -0.728453 & -1.542322 \\
\hline $\mathrm{C}$ & 2.063781 & 1.308866 & -1.407113 \\
\hline $\mathrm{H}$ & 3.061059 & -2.137728 & 0.057354 \\
\hline $\mathrm{H}$ & 4.074272 & -1.355357 & -2.060625 \\
\hline $\mathrm{H}$ & 1.767438 & 2.273281 & -1.816523 \\
\hline $\mathrm{H}$ & 3.394055 & 0.836426 & -3.023103 \\
\hline $\mathrm{C}$ & 1.580704 & -2.639349 & 2.089023 \\
\hline $\mathrm{C}$ & 2.507859 & -5.229631 & 2.600133 \\
\hline $\mathrm{C}$ & 1.219123 & -3.706390 & 1.257928 \\
\hline $\mathrm{C}$ & 2.418024 & -2.886071 & 3.179103 \\
\hline $\mathrm{C}$ & 2.877105 & -4.176996 & 3.431188 \\
\hline $\mathrm{C}$ & 1.678137 & -4.992238 & 1.506391 \\
\hline $\mathrm{H}$ & 0.546400 & -3.527192 & 0.419205 \\
\hline $\mathrm{H}$ & 2.707120 & -2.070346 & 3.841481 \\
\hline $\mathrm{H}$ & 3.524013 & -4.359793 & 4.288139 \\
\hline $\mathrm{H}$ & 1.360697 & -5.809982 & 0.860816 \\
\hline $\mathrm{H}$ & 2.858626 & -6.238948 & 2.809510 \\
\hline $\mathrm{C}$ & -0.998771 & -0.233371 & -2.184190 \\
\hline $\mathrm{C}$ & 0.290970 & -1.292518 & -4.424423 \\
\hline $\mathrm{C}$ & -0.863066 & 0.530466 & -3.347402 \\
\hline $\mathrm{C}$ & -0.490878 & -1.533860 & -2.160185 \\
\hline $\mathrm{C}$ & 0.149875 & -2.064034 & -3.275736 \\
\hline $\mathrm{C}$ & -0.218713 & 0.003979 & -4.460277 \\
\hline $\mathrm{H}$ & -1.253881 & 1.548623 & -3.377243 \\
\hline $\mathrm{H}$ & -0.603023 & -2.126276 & -1.248593 \\
\hline $\mathrm{H}$ & 0.545525 & -3.078070 & -3.240449 \\
\hline $\mathrm{H}$ & -0.112370 & 0.607433 & -5.360639 \\
\hline $\mathrm{H}$ & 0.799593 & -1.701182 & -5.296336 \\
\hline $\mathrm{C}$ & -1.352328 & 2.181334 & -0.707965 \\
\hline $\mathrm{C}$ & -0.543157 & 4.874202 & -0.614511 \\
\hline $\mathrm{C}$ & -0.150090 & 2.561910 & -0.096124 \\
\hline $\mathrm{C}$ & -2.131184 & 3.189789 & -1.285318 \\
\hline $\mathrm{C}$ & -1.733203 & 4.522123 & -1.243744 \\
\hline $\mathrm{C}$ & 0.252165 & 3.890264 & -0.038377 \\
\hline $\mathrm{H}$ & -3.072050 & 2.923565 & -1.767235 \\
\hline $\mathrm{H}$ & -2.358254 & 5.286904 & -1.701137 \\
\hline $\mathrm{H}$ & 1.187508 & 4.128184 & 0.465418 \\
\hline $\mathrm{H}$ & -0.231032 & 5.916066 & -0.571618 \\
\hline $\mathrm{C}$ & -3.600841 & 0.423048 & -1.139534 \\
\hline $\mathrm{C}$ & -6.362679 & 0.135234 & -1.513762 \\
\hline $\mathrm{C}$ & -4.507445 & 1.134732 & -0.342498 \\
\hline $\mathrm{C}$ & -4.097658 & -0.440655 & -2.119759 \\
\hline $\mathrm{C}$ & -5.470498 & -0.585570 & -2.300638 \\
\hline $\mathrm{C}$ & -5.876675 & 1.001839 & -0.536849 \\
\hline $\mathrm{H}$ & -4.134720 & 1.791937 & 0.445960 \\
\hline $\mathrm{H}$ & -3.407472 & -1.016633 & -2.737185 \\
\hline $\mathrm{H}$ & -5.843111 & -1.266928 & -3.063977 \\
\hline $\mathrm{H}$ & -6.568067 & 1.565730 & 0.087565 \\
\hline $\mathrm{H}$ & -7.435616 & 0.019800 & -1.657105 \\
\hline 0 & 0.632598 & 1.616023 & 0.524915 \\
\hline $\mathrm{C}$ & -2.461765 & -2.944107 & 1.908443 \\
\hline $\mathrm{C}$ & -4.476195 & -1.463372 & 2.052459 \\
\hline $\mathrm{C}$ & -3.325849 & -2.038799 & 4.006844 \\
\hline 0 & -4.577562 & -1.934109 & 3.376639 \\
\hline
\end{tabular}

$\begin{array}{cccc}\mathrm{C} & -2.509475 & -3.162265 & 3.386547 \\ \mathrm{C} & -3.423192 & -2.189231 & 1.234304 \\ \mathrm{H} & -4.292334 & -0.370969 & 2.058539 \\ \mathrm{H} & -2.774925 & -1.078949 & 3.925524 \\ \mathrm{H} & -3.003363 & -4.126790 & 3.598796 \\ \mathrm{H} & -3.691987 & -2.429602 & 0.206361 \\ \mathrm{H} & -5.463423 & -1.617576 & 1.596114 \\ \mathrm{H} & -3.518962 & -2.232065 & 5.068432 \\ \mathrm{H} & -1.495002 & -3.204980 & 3.811369 \\ \mathrm{O} & -1.768926 & -4.003149 & 1.218378 \\ \mathrm{~S} & -2.508361 & -5.280329 & 0.608304 \\ \mathrm{O} & -1.692895 & -6.441842 & 0.877316 \\ \mathrm{O} & -3.935022 & -5.251581 & 0.833794 \\ \mathrm{C} & -2.212223 & -4.889956 & -1.169799 \\ \mathrm{~F} & -0.909118 & -4.756633 & -1.380276 \\ \mathrm{~F} & -2.676238 & -5.884742 & -1.902276 \\ \mathrm{~F} & -2.823788 & -3.767371 & -1.513966 \\ -------------------------------------\end{array}$

SCF energy: -3503.209331820

M06-D3 (Gas Phase) /6-31G(d)+LANL2DZ (Pd) opt. No imaginary frequency 


\begin{tabular}{|c|c|c|c|}
\hline \multicolumn{4}{|l|}{ TS-1 } \\
\hline $\mathrm{Pd}$ & 0.518459 & -0.554674 & 0.682283 \\
\hline $\mathrm{P}$ & 1.330983 & 0.278191 & -1.405870 \\
\hline $\mathrm{P}$ & -1.771636 & 0.242489 & 0.836339 \\
\hline $\mathrm{C}$ & 1.628136 & -0.830184 & -2.821046 \\
\hline $\mathrm{C}$ & 2.121271 & -2.605760 & -4.909325 \\
\hline $\mathrm{C}$ & 1.438841 & -0.422492 & -4.144704 \\
\hline $\mathrm{C}$ & 2.074286 & -2.124998 & -2.550402 \\
\hline $\mathrm{C}$ & 2.315541 & -3.012540 & -3.593557 \\
\hline $\mathrm{C}$ & 1.687833 & -1.310227 & -5.184740 \\
\hline $\mathrm{H}$ & 1.092091 & 0.588959 & -4.359864 \\
\hline $\mathrm{H}$ & 2.225475 & -2.448815 & -1.519592 \\
\hline $\mathrm{H}$ & 2.628558 & -4.028793 & -3.363464 \\
\hline $\mathrm{H}$ & 1.536199 & -0.992109 & -6.215304 \\
\hline $\mathrm{H}$ & 2.300293 & -3.302901 & -5.726616 \\
\hline $\mathrm{C}$ & -3.002635 & -0.332479 & -0.403212 \\
\hline $\mathrm{C}$ & -4.786603 & -1.351560 & -2.321366 \\
\hline $\mathrm{C}$ & -2.647952 & -0.420747 & -1.755127 \\
\hline $\mathrm{C}$ & -4.284230 & -0.760188 & -0.040792 \\
\hline $\mathrm{C}$ & -5.172305 & -1.258309 & -0.988008 \\
\hline $\mathrm{C}$ & -3.519789 & -0.930113 & -2.707560 \\
\hline $\mathrm{H}$ & -4.583009 & -0.715194 & 1.006574 \\
\hline $\mathrm{H}$ & -6.162640 & -1.587203 & -0.679174 \\
\hline $\mathrm{H}$ & -3.179439 & -0.997436 & -3.739057 \\
\hline $\mathrm{H}$ & -5.471080 & -1.756305 & -3.064474 \\
\hline $\mathrm{C}$ & -2.479874 & -0.405548 & 2.399297 \\
\hline $\mathrm{C}$ & -3.397520 & -1.518693 & 4.790732 \\
\hline $\mathrm{C}$ & -3.062266 & 0.406204 & 3.373072 \\
\hline $\mathrm{C}$ & -2.363054 & -1.784163 & 2.628158 \\
\hline $\mathrm{C}$ & -2.822892 & -2.334766 & 3.816873 \\
\hline $\mathrm{C}$ & -3.516721 & -0.151967 & 4.566904 \\
\hline $\mathrm{H}$ & -3.159560 & 1.478835 & 3.205177 \\
\hline $\mathrm{H}$ & -1.902303 & -2.423258 & 1.870702 \\
\hline $\mathrm{H}$ & -2.721046 & -3.406033 & 3.984237 \\
\hline $\mathrm{H}$ & -3.966257 & 0.488958 & 5.324015 \\
\hline $\mathrm{H}$ & -3.750683 & -1.950828 & 5.725774 \\
\hline $\mathrm{C}$ & -2.048194 & 2.048947 & 0.938317 \\
\hline $\mathrm{C}$ & -2.323622 & 4.819038 & 1.148460 \\
\hline $\mathrm{C}$ & -0.984162 & 2.833186 & 1.391584 \\
\hline $\mathrm{C}$ & -3.256084 & 2.663969 & 0.599176 \\
\hline $\mathrm{C}$ & -3.390549 & 4.044175 & 0.700545 \\
\hline $\mathrm{C}$ & -1.121657 & 4.212636 & 1.501142 \\
\hline $\mathrm{H}$ & -0.042616 & 2.343450 & 1.653646 \\
\hline $\mathrm{H}$ & -4.090935 & 2.058790 & 0.242511 \\
\hline $\mathrm{H}$ & -4.332395 & 4.517839 & 0.427591 \\
\hline $\mathrm{H}$ & -0.285927 & 4.816100 & 1.851806 \\
\hline $\mathrm{H}$ & -2.430145 & 5.900185 & 1. 222568 \\
\hline $\mathrm{C}$ & 0.263030 & 1.606721 & -2.070827 \\
\hline $\mathrm{C}$ & -1.608413 & 3.561788 & -2.781873 \\
\hline $\mathrm{C}$ & -1.080515 & 1.276370 & -2.292903 \\
\hline $\mathrm{C}$ & 0.652874 & 2.935342 & -2.241638 \\
\hline $\mathrm{C}$ & -0.275718 & 3.911128 & -2.593475 \\
\hline $\mathrm{C}$ & -2.018352 & 2.239186 & -2.643566 \\
\hline $\mathrm{H}$ & 1.694239 & 3.211711 & -2.080444 \\
\hline $\mathrm{H}$ & 0.042399 & 4.945017 & -2.711478 \\
\hline $\mathrm{H}$ & -3.058227 & 1.953267 & -2.794515 \\
\hline $\mathrm{H}$ & -2.342072 & 4.322638 & -3.043058 \\
\hline $\mathrm{C}$ & 2.943282 & 1.119250 & -1.161546 \\
\hline $\mathrm{C}$ & 5.415725 & 2.280264 & -0.546929 \\
\hline $\mathrm{C}$ & 3.069705 & 1.998460 & -0.077898 \\
\hline $\mathrm{C}$ & 4.069840 & 0.834186 & -1.935727 \\
\hline $\mathrm{C}$ & 5.300905 & 1.407849 & -1.623303 \\
\hline $\mathrm{C}$ & 4.292654 & 2.584517 & 0.221143 \\
\hline $\mathrm{H}$ & 2.195749 & 2.208947 & 0.543520 \\
\hline $\mathrm{H}$ & 3.989198 & 0.148708 & -2.778852 \\
\hline $\mathrm{H}$ & 6.174581 & 1.167617 & -2.227026 \\
\hline $\mathrm{H}$ & 4.375121 & 3.267421 & 1.065771 \\
\hline $\mathrm{H}$ & 6.379670 & 2.723859 & -0.303247 \\
\hline $\mathrm{C}$ & 2.124697 & -1.385184 & 1.520562 \\
\hline $\mathrm{C}$ & 1.391603 & -1.432076 & 2.661404 \\
\hline $\mathrm{H}$ & 0.487064 & -2.026950 & 2.772005 \\
\hline $\mathrm{C}$ & 3.581164 & -1.207833 & 1.443857 \\
\hline $\mathrm{H}$ & 4.079878 & -2.179033 & 1.558372 \\
\hline
\end{tabular}

$\begin{array}{crrr}\mathrm{H} & 3.919397 & -0.739171 & 0.509465 \\ \mathrm{C} & 3.923933 & -0.303760 & 2.648138 \\ \mathrm{H} & 3.519796 & 0.711861 & 2.471546 \\ \mathrm{H} & 5.012150 & -0.226444 & 2.756652 \\ \mathrm{O} & 3.423859 & -0.837925 & 3.842226 \\ \mathrm{C} & 2.020373 & -0.812843 & 3.903203 \\ \mathrm{H} & 1.737990 & -1.377511 & 4.799441 \\ \mathrm{H} & 1.661706 & 0.227348 & 4.028864 \\ \mathrm{O} & 1.929711 & -3.354744 & 0.602305 \\ \mathrm{~S} & 0.627223 & -3.928577 & 0.132859 \\ \mathrm{O} & -0.482371 & -2.954765 & 0.152461 \\ \mathrm{O} & 0.747233 & -4.784970 & -1.038334 \\ \mathrm{C} & 0.176057 & -5.060220 & 1.507017 \\ \mathrm{~F} & 1.125773 & -5.956956 & 1.717690 \\ \mathrm{~F} & -0.003898 & -4.361105 & 2.634163 \\ \mathrm{~F} & -0.959278 & -5.684906 & 1.226996 \\ \mathrm{O} & -1.387920 & -0.052148 & -2.167251 \\ --------------------------------------\end{array}$

SCF energy: -3503.176832630

M06-D3 (Gas Phase)/6-31G(d) +LANL2DZ (Pd) opt. 1 imaginary frequency: $142 i \mathrm{~cm}-1$ 


\begin{tabular}{|c|c|c|c|}
\hline \multicolumn{4}{|c|}{ Int-2 } \\
\hline $\mathrm{Pd}$ & 1.992521 & 0.936299 & 1.290071 \\
\hline $\mathrm{P}$ & 2.366757 & 1.046802 & -1.175491 \\
\hline $\mathrm{P}$ & -0.284215 & 1.190584 & 1.490523 \\
\hline $\mathrm{C}$ & 1.416033 & -0.200583 & -2.112091 \\
\hline $\mathrm{C}$ & 0.050114 & -2.242747 & -3.429823 \\
\hline $\mathrm{C}$ & 0.876322 & 0.022285 & -3.379227 \\
\hline $\mathrm{C}$ & 1.284204 & -1.459148 & -1.512509 \\
\hline $\mathrm{C}$ & 0.604137 & -2.475208 & -2.173420 \\
\hline $\mathrm{C}$ & 0.190295 & -0.996721 & -4.033394 \\
\hline $\mathrm{H}$ & 0.990764 & 0.996734 & -3.856133 \\
\hline $\mathrm{H}$ & 1.737954 & -1.650242 & -0.535939 \\
\hline $\mathrm{H}$ & 0.510114 & -3.452506 & -1.703197 \\
\hline $\mathrm{H}$ & -0.236097 & -0.814842 & -5.019155 \\
\hline $\mathrm{H}$ & -0.487649 & -3.038156 & -3.943755 \\
\hline $\mathrm{C}$ & -1.158286 & 1.203821 & -0.121493 \\
\hline $\mathrm{C}$ & -2.354740 & 1.242384 & -2.654240 \\
\hline $\mathrm{C}$ & -0.810547 & 2.168329 & -1.071555 \\
\hline $\mathrm{C}$ & -2.106234 & 0.241032 & -0.479752 \\
\hline $\mathrm{C}$ & -2.696213 & 0.253569 & -1.738714 \\
\hline $\mathrm{C}$ & -1.411098 & 2.207688 & -2.324596 \\
\hline $\mathrm{H}$ & -2.375487 & -0.538832 & 0.230291 \\
\hline $\mathrm{H}$ & -3.420855 & -0.513651 & -2.002656 \\
\hline $\mathrm{H}$ & -1.122209 & 2.978689 & -3.036974 \\
\hline $\mathrm{H}$ & -2.816494 & 1.260238 & -3.640068 \\
\hline $\mathrm{C}$ & -1.022560 & -0.275036 & 2.288176 \\
\hline $\mathrm{C}$ & -2.195433 & -2.592553 & 3.299975 \\
\hline $\mathrm{C}$ & -2.239219 & -0.203988 & 2.971659 \\
\hline $\mathrm{C}$ & -0.393197 & -1.512201 & 2.115891 \\
\hline $\mathrm{C}$ & -0.982773 & -2.666620 & 2.620601 \\
\hline $\mathrm{C}$ & -2.821333 & -1.361544 & 3.477718 \\
\hline $\mathrm{H}$ & -2.736376 & 0.757428 & 3.103192 \\
\hline $\mathrm{H}$ & 0.578822 & -1.570959 & 1.621373 \\
\hline $\mathrm{H}$ & -0.480711 & -3.623589 & 2.491282 \\
\hline $\mathrm{H}$ & -3.768189 & -1.301124 & 4.011845 \\
\hline $\mathrm{H}$ & -2.652628 & -3.496888 & 3.698542 \\
\hline $\mathrm{C}$ & -0.993238 & 2.613371 & 2.402930 \\
\hline $\mathrm{C}$ & -2.053240 & 4.744210 & 3.874897 \\
\hline $\mathrm{C}$ & -0.697818 & 2.754230 & 3.764354 \\
\hline $\mathrm{C}$ & -1.838433 & 3.543092 & 1.793261 \\
\hline $\mathrm{C}$ & -2.361325 & 4.604111 & 2.526482 \\
\hline $\mathrm{C}$ & -1.224352 & 3.812369 & 4.493806 \\
\hline $\mathrm{H}$ & -0.055576 & 2.019849 & 4.250249 \\
\hline $\mathrm{H}$ & -2.101342 & 3.440422 & 0.742453 \\
\hline $\mathrm{H}$ & -3.017862 & 5.322021 & 2.037619 \\
\hline $\mathrm{H}$ & -0.985081 & 3.906081 & 5.552260 \\
\hline $\mathrm{H}$ & -2.463480 & 5.575652 & 4.445684 \\
\hline $\mathrm{C}$ & 2.075935 & 2.656457 & -2.035012 \\
\hline C & 1.767964 & 5.225196 & -3.150754 \\
\hline $\mathrm{C}$ & 1.031633 & 3.505221 & -1.646750 \\
\hline $\mathrm{C}$ & 2.948099 & 3.127594 & -3.024061 \\
\hline $\mathrm{C}$ & 2.793893 & 4. 391643 & -3.583349 \\
\hline $\mathrm{C}$ & 0.879515 & 4.778332 & -2.179491 \\
\hline $\mathrm{H}$ & 3.773569 & 2.494472 & -3.347584 \\
\hline $\mathrm{H}$ & 3.488265 & 4.729007 & -4.350315 \\
\hline $\mathrm{H}$ & 0.058879 & 5.397132 & -1.820712 \\
\hline $\mathrm{H}$ & 1.653280 & 6.222266 & -3.571881 \\
\hline $\mathrm{C}$ & 4.098180 & 0.712497 & -1.651671 \\
\hline $\mathrm{C}$ & 6.773350 & 0.325962 & -2.326468 \\
\hline $\mathrm{C}$ & 5.101386 & 1.430124 & -0.992343 \\
\hline $\mathrm{C}$ & 4.443385 & -0.200482 & -2.648014 \\
\hline $\mathrm{C}$ & 5.780736 & -0.393541 & -2.980848 \\
\hline $\mathrm{C}$ & 6.432558 & 1.241860 & -1.334022 \\
\hline $\mathrm{H}$ & 4.836445 & 2.124825 & -0.195003 \\
\hline $\mathrm{H}$ & 3.670318 & -0.771837 & -3.160773 \\
\hline $\mathrm{H}$ & 6.045357 & -1.115986 & -3.751046 \\
\hline $\mathrm{H}$ & 7.208927 & 1.797063 & -0.810793 \\
\hline $\mathrm{H}$ & 7.819481 & 0.167299 & -2.583468 \\
\hline $\mathrm{C}$ & 2.042170 & 1.027817 & 3.320620 \\
\hline $\mathrm{C}$ & 1.764764 & 0.062271 & 4.189765 \\
\hline $\mathrm{H}$ & 1.254580 & -0.858830 & 3.905425 \\
\hline $\mathrm{C}$ & 2.759894 & 2.278729 & 3.728364 \\
\hline $\mathrm{H}$ & 3.792578 & 2.244893 & 3.346432 \\
\hline
\end{tabular}

\begin{tabular}{|c|c|c|c|}
\hline $\mathrm{H}$ & 2.284837 & 3.175560 & 3.296460 \\
\hline C & 2.762307 & 2.393141 & 5.252427 \\
\hline $\mathrm{H}$ & 1.754758 & 2.697097 & 5.605044 \\
\hline $\mathrm{H}$ & 3.477049 & 3.153588 & 5.590548 \\
\hline 0 & 3.140888 & 1.184587 & 5.854594 \\
\hline C & 2.200943 & 0.166260 & 5.627667 \\
\hline $\mathrm{H}$ & 2.679434 & -0.771007 & 5.941542 \\
\hline $\mathrm{H}$ & 1.314100 & 0.315736 & 6.280801 \\
\hline O & 4.081897 & 0.217665 & 1.504726 \\
\hline S & 4.162165 & -1.284981 & 1.402670 \\
\hline 0 & 2.855081 & -1.937321 & 1.574117 \\
\hline 0 & 5.000455 & -1.762523 & 0.312655 \\
\hline $\mathrm{C}$ & 5.093142 & -1.689353 & 2.931904 \\
\hline F & 6.294081 & -1.129170 & 2.890782 \\
\hline F & 4.452663 & -1.244565 & 4.005431 \\
\hline F & 5.233380 & -3.005115 & 3.033170 \\
\hline 0 & 0.128391 & 3.095986 & -0.687744 \\
\hline
\end{tabular}

SCF energy: -3503.214167890

M06-D3 (Gas Phase) /6-31G(d)+LANL2DZ (Pd) opt. No imaginary frequency 


\begin{tabular}{|c|c|c|c|}
\hline \multicolumn{4}{|c|}{ Int-3 } \\
\hline $\mathrm{Pd}$ & 1.348414 & 0.950019 & 1.505657 \\
\hline $\mathrm{P}$ & 1.697228 & 0.723484 & -1.028929 \\
\hline $\mathrm{P}$ & -0.989142 & 0.895348 & 1.724718 \\
\hline $\mathrm{C}$ & 1.202373 & -0.838202 & -1.841770 \\
\hline $\mathrm{C}$ & 0.446943 & -3.264273 & -2.993631 \\
\hline $\mathrm{C}$ & 1.068331 & -0.947063 & -3.230897 \\
\hline $\mathrm{C}$ & 0.960763 & -1.953542 & -1.038618 \\
\hline $\mathrm{C}$ & 0.583691 & -3.163529 & -1.613910 \\
\hline $\mathrm{C}$ & 0.693695 & -2.155887 & -3.802566 \\
\hline $\mathrm{H}$ & 1.249278 & -0.079890 & -3.867152 \\
\hline $\mathrm{H}$ & 1.076024 & -1.877002 & 0.044714 \\
\hline $\mathrm{H}$ & 0.394319 & -4.026105 & -0.976724 \\
\hline $\mathrm{H}$ & 0.591114 & -2.234695 & -4.883339 \\
\hline $\mathrm{H}$ & 0.147962 & -4.209076 & -3.444114 \\
\hline $\mathrm{C}$ & -1.762987 & 0.098194 & 0.275773 \\
\hline $\mathrm{C}$ & -2.721044 & -0.988095 & -2.110620 \\
\hline $\mathrm{C}$ & -1.631077 & 0.784419 & -0.935146 \\
\hline $\mathrm{C}$ & -2.426872 & -1.130491 & 0.276791 \\
\hline $\mathrm{C}$ & -2.902454 & -1.672598 & -0.912563 \\
\hline $\mathrm{C}$ & -2.095240 & 0.253988 & -2.130538 \\
\hline $\mathrm{H}$ & -2.570210 & -1.670180 & 1. 210938 \\
\hline $\mathrm{H}$ & -3.410932 & -2.633990 & -0.903061 \\
\hline $\mathrm{H}$ & -1.961613 & 0.802781 & -3.061161 \\
\hline $\mathrm{H}$ & -3.079663 & -1.418113 & -3.043793 \\
\hline $\mathrm{C}$ & -1.468568 & -0.187531 & 3.113454 \\
\hline $\mathrm{C}$ & -1.997485 & -1.852581 & 5.297242 \\
\hline $\mathrm{C}$ & -2.232200 & 0.277494 & 4.186765 \\
\hline $\mathrm{C}$ & -0.971512 & -1.496461 & 3.146022 \\
\hline $\mathrm{C}$ & -1.236936 & -2.325076 & 4.229459 \\
\hline $\mathrm{C}$ & -2.495375 & -0.554428 & 5.271733 \\
\hline $\mathrm{H}$ & -2.610032 & 1.298859 & 4.186711 \\
\hline $\mathrm{H}$ & -0.365086 & -1.867863 & 2.317793 \\
\hline $\mathrm{H}$ & -0.849278 & -3.342645 & 4.243009 \\
\hline $\mathrm{H}$ & -3.088915 & -0.180589 & 6.103700 \\
\hline $\mathrm{H}$ & -2.201101 & -2.498644 & 6.148869 \\
\hline $\mathrm{C}$ & -1.972304 & 2.414662 & 1.950072 \\
\hline $\mathrm{C}$ & -3.523612 & 4.704384 & 2.299430 \\
\hline $\mathrm{C}$ & -1.365753 & 3.636035 & 2. 234791 \\
\hline $\mathrm{C}$ & -3.364369 & 2.346468 & 1.824164 \\
\hline $\mathrm{C}$ & -4.135339 & 3.488270 & 2.003643 \\
\hline $\mathrm{C}$ & -2.139284 & 4.778589 & 2.411084 \\
\hline $\mathrm{H}$ & -0.282704 & 3.694135 & 2.304610 \\
\hline $\mathrm{H}$ & -3.846667 & 1.396921 & 1.587935 \\
\hline $\mathrm{H}$ & -5.217858 & 3.429231 & 1.909121 \\
\hline $\mathrm{H}$ & -1.657124 & 5.729183 & 2.632078 \\
\hline $\mathrm{H}$ & -4.129698 & 5.597679 & 2.437685 \\
\hline $\mathrm{C}$ & 0.971277 & 2.019512 & -2.132364 \\
\hline C & -0.181878 & 4.015718 & -3.752710 \\
\hline $\mathrm{C}$ & -0.320310 & 2.501115 & -1.900131 \\
\hline $\mathrm{C}$ & 1.663525 & 2.554306 & -3.227176 \\
\hline $\mathrm{C}$ & 1.094479 & 3.536306 & -4.030614 \\
\hline $\mathrm{C}$ & -0.896351 & 3.492839 & -2.681879 \\
\hline $\mathrm{H}$ & 2.669957 & 2.203863 & -3.451091 \\
\hline $\mathrm{H}$ & 1.656473 & 3.932127 & -4.873823 \\
\hline $\mathrm{H}$ & -1.898836 & 3.836111 & -2.433599 \\
\hline $\mathrm{H}$ & -0.624766 & 4.792504 & -4.372435 \\
\hline $\mathrm{C}$ & 3.466854 & 0.931660 & -1.450885 \\
\hline $\mathrm{C}$ & 6.188378 & 1.334199 & -1.938705 \\
\hline $\mathrm{C}$ & 4.068848 & 2.161336 & -1.148288 \\
\hline $\mathrm{C}$ & 4.239742 & -0.091663 & -2.000537 \\
\hline $\mathrm{C}$ & 5.599079 & 0.110293 & -2.236849 \\
\hline $\mathrm{C}$ & 5.418654 & 2.365250 & -1.400103 \\
\hline $\mathrm{H}$ & 3.471026 & 2.964515 & -0.709158 \\
\hline $\mathrm{H}$ & 3.781369 & -1.051244 & -2.241955 \\
\hline $\mathrm{H}$ & 6.196451 & -0.692858 & -2.664807 \\
\hline $\mathrm{H}$ & 5.875252 & 3.325987 & -1.169623 \\
\hline $\mathrm{H}$ & 7.248373 & 1.489348 & -2.129841 \\
\hline $\mathrm{C}$ & 1.318478 & 1.867836 & 3. 309555 \\
\hline $\mathrm{C}$ & 0.933155 & 1.445156 & 4.508381 \\
\hline $\mathrm{H}$ & 0.367019 & 0.524234 & 4.658398 \\
\hline $\mathrm{C}$ & 2.088806 & 3.149139 & 3.133210 \\
\hline $\mathrm{H}$ & 3.154259 & 2.940691 & 2.940455 \\
\hline
\end{tabular}

\begin{tabular}{|c|c|c|c|}
\hline $\mathrm{H}$ & 1.737546 & 3.734063 & 2.267829 \\
\hline C & 1.959841 & 3.980117 & 4.412809 \\
\hline $\mathrm{H}$ & 0.930010 & 4.379054 & 4.495045 \\
\hline $\mathrm{H}$ & 2.657637 & 4.825149 & 4.408230 \\
\hline 0 & 2.272597 & 3.192857 & 5.530633 \\
\hline C & 1.305583 & 2.201031 & 5.757651 \\
\hline $\mathrm{H}$ & 1.733648 & 1.515141 & 6.502130 \\
\hline $\mathrm{H}$ & 0.395447 & 2.643933 & 6.209924 \\
\hline 0 & -1.041032 & 2.016432 & -0.833313 \\
\hline $\mathrm{N}$ & 3.539823 & -0.027982 & 2.338841 \\
\hline $\mathrm{C}$ & 3.707298 & 0.002878 & 3.806114 \\
\hline $\mathrm{H}$ & 2.852361 & -0.439733 & 4.318642 \\
\hline $\mathrm{H}$ & 3.798662 & 1.039976 & 4.146184 \\
\hline $\mathrm{H}$ & 4.618886 & -0.551040 & 4.083466 \\
\hline C & 4.711877 & 0.639131 & 1.764908 \\
\hline $\mathrm{H}$ & 4.653263 & 0.663761 & 0.677688 \\
\hline $\mathrm{H}$ & 5.646304 & 0.136715 & 2.068108 \\
\hline $\mathrm{H}$ & 4.741729 & 1.672499 & 2.128740 \\
\hline $\mathrm{C}$ & 3.351748 & -1.380541 & 1.874458 \\
\hline C & 2.905338 & -4.026084 & 1.028323 \\
\hline C & 4.138252 & -1.949485 & 0.870294 \\
\hline C & 2.340665 & -2.159774 & 2.452897 \\
\hline C & 2.123699 & -3.465772 & 2.036325 \\
\hline $\mathrm{C}$ & 3.905270 & -3.257457 & 0.448852 \\
\hline $\mathrm{H}$ & 4.940407 & -1.387094 & 0.401143 \\
\hline $\mathrm{H}$ & 1.713947 & -1.739559 & 3.237846 \\
\hline $\mathrm{H}$ & 1.337002 & -4.053288 & 2.509594 \\
\hline $\mathrm{H}$ & 4.529358 & -3.675946 & -0.339107 \\
\hline $\mathrm{H}$ & 2.738125 & -5.050956 & 0.703491 \\
\hline
\end{tabular}

SCF energy: -2907.745937950

M06-D3 (Gas Phase) /6-31G(d) +LANL2DZ (Pd) opt. No imaginary frequency 


\begin{tabular}{|c|c|c|c|}
\hline \multicolumn{4}{|c|}{ Int-4 } \\
\hline $\mathrm{Pd}$ & 0.696929 & 0.193118 & 0.586641 \\
\hline $\mathrm{P}$ & 1.061819 & 0.094152 & -1.822258 \\
\hline $\mathrm{P}$ & -1.557485 & 0.301256 & 1.036695 \\
\hline $\mathrm{C}$ & 0.185179 & -1.050672 & -2.935677 \\
\hline $\mathrm{C}$ & -1.136885 & -2.876858 & -4.576075 \\
\hline $\mathrm{C}$ & 0.095287 & -0.821463 & -4.312901 \\
\hline $\mathrm{C}$ & -0.394563 & -2.197343 & -2.388301 \\
\hline $\mathrm{C}$ & -1.052781 & -3.109141 & -3.206938 \\
\hline $\mathrm{C}$ & -0.562753 & -1.733676 & -5.128737 \\
\hline $\mathrm{H}$ & 0.535508 & 0.077563 & -4.746458 \\
\hline $\mathrm{H}$ & -0.336370 & -2.369666 & -1.312234 \\
\hline $\mathrm{H}$ & -1.505651 & -3.998844 & -2.773544 \\
\hline $\mathrm{H}$ & -0.628934 & -1.552548 & -6.199886 \\
\hline $\mathrm{H}$ & -1.653861 & -3.588413 & -5.217147 \\
\hline $\mathrm{C}$ & -2.410861 & 0.254374 & -0.574109 \\
\hline $\mathrm{C}$ & -3.519228 & 0.074324 & -3.133906 \\
\hline $\mathrm{C}$ & -2.030269 & 1.144536 & -1.588601 \\
\hline $\mathrm{C}$ & -3.367792 & -0.722804 & -0.868896 \\
\hline $\mathrm{C}$ & -3.923246 & -0.810776 & -2.139564 \\
\hline $\mathrm{C}$ & -2.576526 & 1.059705 & -2.863030 \\
\hline $\mathrm{H}$ & -3.671848 & -1.430141 & -0.099035 \\
\hline $\mathrm{H}$ & -4.662639 & -1.578548 & -2.354786 \\
\hline $\mathrm{H}$ & -2.256299 & 1.757661 & -3.634836 \\
\hline $\mathrm{H}$ & -3.941209 & 0.001921 & -4.134293 \\
\hline $\mathrm{C}$ & -2.134565 & -1.189732 & 1.897078 \\
\hline $\mathrm{C}$ & -3.065157 & -3.548688 & 3.053272 \\
\hline $\mathrm{C}$ & -3.308531 & -1.181500 & 2.656377 \\
\hline $\mathrm{C}$ & -1.431002 & -2.386077 & 1.720491 \\
\hline $\mathrm{C}$ & -1.896245 & -3.562471 & 2.296058 \\
\hline $\mathrm{C}$ & -3.768784 & -2.361119 & 3. 232385 \\
\hline $\mathrm{H}$ & -3.866844 & -0.255595 & 2.792572 \\
\hline $\mathrm{H}$ & -0.507686 & -2.386236 & 1.135762 \\
\hline $\mathrm{H}$ & -1.343306 & -4.489528 & 2.158728 \\
\hline $\mathrm{H}$ & -4.682723 & -2.351664 & 3.822776 \\
\hline $\mathrm{H}$ & -3.428105 & -4.468312 & 3.507907 \\
\hline $\mathrm{C}$ & -2.233726 & 1.717034 & 1.953888 \\
\hline $\mathrm{C}$ & -3.270379 & 3.861947 & 3.407229 \\
\hline $\mathrm{C}$ & -1.967291 & 1.837063 & 3. 324364 \\
\hline $\mathrm{C}$ & -3.028839 & 2.675899 & 1. 321034 \\
\hline $\mathrm{C}$ & -3.541823 & 3.744954 & 2.048536 \\
\hline $\mathrm{C}$ & -2.486754 & 2.903152 & 4.046035 \\
\hline $\mathrm{H}$ & -1.354622 & 1.086788 & 3.825661 \\
\hline $\mathrm{H}$ & -3.259441 & 2.586368 & 0.261681 \\
\hline $\mathrm{H}$ & -4.162426 & 4.486115 & 1.549157 \\
\hline $\mathrm{H}$ & -2.279412 & 2.985059 & 5.111584 \\
\hline $\mathrm{H}$ & -3.673746 & 4.699455 & 3.972899 \\
\hline $\mathrm{C}$ & 0.856314 & 1.750332 & -2.579812 \\
\hline $\mathrm{C}$ & 0.536505 & 4.337797 & -3.615635 \\
\hline $\mathrm{C}$ & -0.222920 & 2.558269 & -2.198132 \\
\hline $\mathrm{C}$ & 1.773942 & 2.273867 & -3.496297 \\
\hline $\mathrm{C}$ & 1.614501 & 3.554335 & -4.015557 \\
\hline $\mathrm{C}$ & -0.387642 & 3.839813 & -2.702402 \\
\hline $\mathrm{H}$ & 2.632410 & 1.671197 & -3.794471 \\
\hline $\mathrm{H}$ & 2.339276 & 3.943114 & -4.727391 \\
\hline $\mathrm{H}$ & -1.235897 & 4.432194 & -2.365013 \\
\hline $\mathrm{H}$ & 0.412719 & 5.342996 & -4.012916 \\
\hline $\mathrm{C}$ & 2.839604 & -0.252375 & -2.032402 \\
\hline $\mathrm{C}$ & 5.585144 & -0.723430 & -2.161967 \\
\hline $\mathrm{C}$ & 3.711238 & 0.387952 & -1.141366 \\
\hline $\mathrm{C}$ & 3.352802 & -1.134656 & -2.984228 \\
\hline $\mathrm{C}$ & 4.724382 & -1.367290 & -3.045072 \\
\hline $\mathrm{C}$ & 5.078962 & 0.157730 & -1.208501 \\
\hline $\mathrm{H}$ & 3.314625 & 1.089989 & -0.399909 \\
\hline $\mathrm{H}$ & 2.682401 & -1.643690 & -3.676349 \\
\hline $\mathrm{H}$ & 5.121383 & -2.056345 & -3.787999 \\
\hline $\mathrm{H}$ & 5.750470 & 0.661647 & -0.516234 \\
\hline $\mathrm{H}$ & 6.655944 & -0.910058 & -2.213663 \\
\hline $\mathrm{C}$ & 0.901960 & 0.506024 & 2.561469 \\
\hline $\mathrm{C}$ & 0.953604 & -0.481233 & 3.454810 \\
\hline $\mathrm{H}$ & 0.512578 & -1.464591 & 3.279402 \\
\hline $\mathrm{C}$ & 1.360201 & 1.901555 & 2.860508 \\
\hline $\mathrm{H}$ & 2. 279031 & 2.144096 & 2.303861 \\
\hline
\end{tabular}

$\begin{array}{cccc}\mathrm{H} & 0.598398 & 2.637205 & 2.554949 \\ \mathrm{C} & 1.608922 & 2.023198 & 4.368521 \\ \mathrm{H} & 0.635776 & 2.085341 & 4.893982 \\ \mathrm{H} & 2.180958 & 2.927544 & 4.602453 \\ \mathrm{O} & 2.351399 & 0.933061 & 4.845373 \\ \mathrm{C} & 1.631208 & -0.265018 & 4.784888 \\ \mathrm{H} & 2.343901 & -1.073563 & 4.991312 \\ \mathrm{H} & 0.861479 & -0.298069 & 5.584327 \\ \mathrm{O} & -1.120718 & 2.111866 & -1.244881 \\ -\end{array}$

SCF energy: -2541.782761850

M06-D3 (Gas Phase)/6-31G(d)+LANL2DZ (Pd) opt. No imaginary frequency 


\begin{tabular}{|c|c|c|c|}
\hline \multicolumn{4}{|l|}{ Int-5 } \\
\hline $\mathrm{Pd}$ & -0.000023 & -0.178097 & 0.232429 \\
\hline $\mathrm{P}$ & 0.366412 & -0.272861 & -2.213919 \\
\hline $\mathrm{P}$ & -2.302533 & 0.056848 & 0.482557 \\
\hline $\mathrm{C}$ & -0.779860 & -1.190235 & -3.296974 \\
\hline $\mathrm{C}$ & -2.409150 & -2.757472 & -4.929347 \\
\hline $\mathrm{C}$ & -0.934023 & -0.870663 & -4.649377 \\
\hline $\mathrm{C}$ & -1.446964 & -2.298558 & -2.770159 \\
\hline $\mathrm{C}$ & -2.258780 & -3.081356 & -3.584599 \\
\hline $\mathrm{C}$ & -1.748642 & -1.651556 & -5.460442 \\
\hline $\mathrm{H}$ & -0.415329 & -0.006950 & -5.067752 \\
\hline $\mathrm{H}$ & -1.331708 & -2.548310 & -1.713629 \\
\hline $\mathrm{H}$ & -2.776086 & -3.942561 & -3.165841 \\
\hline $\mathrm{H}$ & -1.866207 & -1.398898 & -6.512591 \\
\hline $\mathrm{H}$ & -3.044692 & -3.368162 & -5.567958 \\
\hline $\mathrm{C}$ & -3.101248 & 0.384630 & -1.130895 \\
\hline C & -4.169732 & 0.840764 & -3.678323 \\
\hline $\mathrm{C}$ & -2.548042 & 1.344838 & -1.985426 \\
\hline $\mathrm{C}$ & -4.197431 & -0.352020 & -1.590076 \\
\hline $\mathrm{C}$ & -4.731194 & -0.127097 & -2.852583 \\
\hline $\mathrm{C}$ & -3.076418 & 1.582404 & -3.248295 \\
\hline $\mathrm{H}$ & -4.625271 & -1.131547 & -0.963046 \\
\hline $\mathrm{H}$ & -5.579014 & -0.716676 & -3.193634 \\
\hline $\mathrm{H}$ & -2.622962 & 2.334434 & -3.891358 \\
\hline $\mathrm{H}$ & -4.579447 & 1.019388 & -4.670436 \\
\hline $\mathrm{C}$ & -2.984465 & -1.537908 & 1.037958 \\
\hline $\mathrm{C}$ & -3.999433 & -4.029418 & 1.789063 \\
\hline $\mathrm{C}$ & -4.318457 & -1.663617 & 1.447429 \\
\hline $\mathrm{C}$ & -2.165946 & -2.671450 & 1.018014 \\
\hline $\mathrm{C}$ & -2.671771 & -3.912984 & 1.389862 \\
\hline $\mathrm{C}$ & -4.820964 & -2.905026 & 1.819126 \\
\hline $\mathrm{H}$ & -4.966297 & -0.786997 & 1.478423 \\
\hline $\mathrm{H}$ & -1.118818 & -2.571870 & 0.719241 \\
\hline $\mathrm{H}$ & -2.024641 & -4.787637 & 1.374368 \\
\hline $\mathrm{H}$ & -5.858170 & -2.994152 & 2.135848 \\
\hline $\mathrm{H}$ & -4.395695 & -4.999079 & 2.084016 \\
\hline $\mathrm{C}$ & -2.986141 & 1.316492 & 1.613702 \\
\hline $\mathrm{C}$ & -3.937530 & 3.273112 & 3.369182 \\
\hline $\mathrm{C}$ & -3.306303 & 0.986645 & 2.936037 \\
\hline $\mathrm{C}$ & -3.135338 & 2.639449 & 1.183425 \\
\hline $\mathrm{C}$ & -3.604471 & 3.610977 & 2.061408 \\
\hline $\mathrm{C}$ & -3.790933 & 1.958555 & 3.803751 \\
\hline $\mathrm{H}$ & -3.182401 & -0.035483 & 3.290779 \\
\hline $\mathrm{H}$ & -2.874268 & 2.920013 & 0.164831 \\
\hline $\mathrm{H}$ & -3.716600 & 4.636992 & 1.716678 \\
\hline $\mathrm{H}$ & -4.049601 & 1.686240 & 4.825498 \\
\hline $\mathrm{H}$ & -4.311811 & 4.034171 & 4.050943 \\
\hline $\mathrm{C}$ & 0.417184 & 1.439890 & -2.883424 \\
\hline C & 0.565620 & 4.117873 & -3.729942 \\
\hline $\mathrm{C}$ & -0.484921 & 2.405921 & -2.416851 \\
\hline $\mathrm{C}$ & 1.381085 & 1.851708 & -3.811129 \\
\hline $\mathrm{C}$ & 1.452772 & 3.173871 & -4.236485 \\
\hline $\mathrm{C}$ & -0.411661 & 3.731623 & -2.819117 \\
\hline $\mathrm{H}$ & 2.096573 & 1.125140 & -4.194844 \\
\hline $\mathrm{H}$ & 2.211275 & 3.467083 & -4.958986 \\
\hline $\mathrm{H}$ & -1.129908 & 4.442139 & -2.414160 \\
\hline $\mathrm{H}$ & 0.626322 & 5.156178 & -4.049056 \\
\hline $\mathrm{C}$ & 2.013673 & -0.930670 & -2.668144 \\
\hline $\mathrm{C}$ & 4.563398 & -1.910831 & -3.258327 \\
\hline $\mathrm{C}$ & 3.150826 & -0.324952 & -2.114940 \\
\hline $\mathrm{C}$ & 2.166679 & -2.035794 & -3.507179 \\
\hline $\mathrm{C}$ & 3.439075 & -2.526084 & -3.794011 \\
\hline $\mathrm{C}$ & 4.417042 & -0.805747 & -2.421133 \\
\hline $\mathrm{H}$ & 3.047825 & 0.537228 & -1.451522 \\
\hline $\mathrm{H}$ & 1.293561 & -2.518517 & -3.944565 \\
\hline $\mathrm{H}$ & 3.546981 & -3.389498 & -4.447610 \\
\hline $\mathrm{H}$ & 5.295860 & -0.311949 & -2.008850 \\
\hline $\mathrm{H}$ & 5.556544 & -2.289216 & -3.492262 \\
\hline $\mathrm{C}$ & -0.082224 & 0.290401 & 2.219143 \\
\hline $\mathrm{C}$ & -0.245579 & -0.558794 & 3.231298 \\
\hline $\mathrm{H}$ & -0.544597 & -1.599398 & 3.088584 \\
\hline $\mathrm{C}$ & 0.261117 & 1.736111 & 2.417879 \\
\hline $\mathrm{H}$ & 1.297857 & 1.917726 & 2.095681 \\
\hline
\end{tabular}

\begin{tabular}{|c|c|c|c|}
\hline $\mathrm{H}$ & -0.381336 & 2.384560 & 1.799133 \\
\hline $\mathrm{C}$ & 0.076552 & 2.105090 & 3.890946 \\
\hline $\mathrm{H}$ & -1.000280 & 2.217839 & 4.113229 \\
\hline $\mathrm{H}$ & 0.577952 & 3.050806 & 4.126868 \\
\hline O & 0.644761 & 1.126521 & 4.731066 \\
\hline C & -0.040836 & -0.095217 & 4.652091 \\
\hline $\mathrm{H}$ & 0.554249 & -0.825586 & 5.216936 \\
\hline $\mathrm{H}$ & -1.024470 & -0.015696 & 5.160281 \\
\hline O & -1.468086 & 2.056650 & -1.508223 \\
\hline 0 & 3.057390 & 0.556966 & 1.185848 \\
\hline B & 2.575872 & -0.707830 & 1.108099 \\
\hline O & 3.193296 & -1.628422 & 1.871816 \\
\hline $\mathrm{C}$ & 4.102227 & -0.887358 & 2.740323 \\
\hline C & 4.283194 & 0.466084 & 1.973673 \\
\hline $\mathrm{H}$ & 1.712450 & -1.045727 & 0.317660 \\
\hline C & 5.422505 & 0.442752 & 0.969480 \\
\hline $\mathrm{H}$ & 5.360726 & -0.440458 & 0.319138 \\
\hline $\mathrm{H}$ & 6.400257 & 0.440613 & 1.466563 \\
\hline $\mathrm{H}$ & 5.360929 & 1.339097 & 0.338484 \\
\hline C & 4.375545 & 1.690019 & 2.858159 \\
\hline $\mathrm{H}$ & 3.492337 & 1.802277 & 3.498119 \\
\hline $\mathrm{H}$ & 4.467468 & 2.588972 & 2.236034 \\
\hline $\mathrm{H}$ & 5.263901 & 1.632796 & 3.501867 \\
\hline C & 5.369227 & -1.697605 & 2.905707 \\
\hline $\mathrm{H}$ & 5.816208 & -1.967624 & 1.943385 \\
\hline $\mathrm{H}$ & 5.144102 & -2.624727 & 3.446068 \\
\hline $\mathrm{H}$ & 6.109123 & -1.136524 & 3.492846 \\
\hline $\mathrm{C}$ & 3.395790 & -0.734108 & 4.074033 \\
\hline $\mathrm{H}$ & 2.499569 & -0.105906 & 3.997576 \\
\hline $\mathrm{H}$ & 4.058724 & -0.288237 & 4.826314 \\
\hline $\mathrm{H}$ & 3.096668 & -1.728777 & 4.428525 \\
\hline
\end{tabular}

SCF energy: -2953.418653960

M06-D3 (Gas Phase) /6-31G(d)+LANL2DZ (Pd) opt. No imaginary frequency 


\begin{tabular}{|c|c|c|c|}
\hline \multicolumn{4}{|l|}{ TS-2 } \\
\hline $\mathrm{Pd}$ & 0.251531 & 0.361318 & 0.133723 \\
\hline $\mathrm{P}$ & 0.483962 & 0.017824 & -2.238014 \\
\hline $\mathrm{P}$ & -2.098903 & 0.168520 & 0.339367 \\
\hline $\mathrm{C}$ & -0.639284 & -1.186171 & -3.012246 \\
\hline $\mathrm{C}$ & -2.317511 & -3.130098 & -4.092428 \\
\hline $\mathrm{C}$ & -1.246595 & -0.972699 & -4.250396 \\
\hline $\mathrm{C}$ & -0.857970 & -2.387356 & -2.330064 \\
\hline $\mathrm{C}$ & -1.695236 & -3.356385 & -2.866734 \\
\hline $\mathrm{C}$ & -2.089549 & -1.943716 & -4.783282 \\
\hline $\mathrm{H}$ & -1.069595 & -0.044919 & -4.795103 \\
\hline $\mathrm{H}$ & -0.369199 & -2.561159 & -1.370335 \\
\hline $\mathrm{H}$ & -1.866526 & -4.284213 & -2.323567 \\
\hline $\mathrm{H}$ & -2.571451 & -1.770045 & -5.743826 \\
\hline $\mathrm{H}$ & -2.980703 & -3.883740 & -4.512879 \\
\hline $\mathrm{C}$ & -3.027117 & 0.350917 & -1.232395 \\
\hline $\mathrm{C}$ & -4.364626 & 0.715462 & -3.670210 \\
\hline $\mathrm{C}$ & -2.650735 & 1.363220 & -2.122360 \\
\hline $\mathrm{C}$ & -4.070060 & -0.498653 & -1.614108 \\
\hline $\mathrm{C}$ & -4.733989 & -0.322627 & -2.822050 \\
\hline $\mathrm{C}$ & -3.317511 & 1.560077 & -3.325230 \\
\hline $\mathrm{H}$ & -4.351657 & -1.329425 & -0.971057 \\
\hline $\mathrm{H}$ & -5.538066 & -1.000596 & -3.099166 \\
\hline $\mathrm{H}$ & -3.005805 & 2. 363511 & -3.989873 \\
\hline $\mathrm{H}$ & -4.887219 & 0.866944 & -4.612658 \\
\hline $\mathrm{C}$ & -2.504608 & -1.483728 & 0.992017 \\
\hline $\mathrm{C}$ & -3.032004 & -4.056192 & 1.951262 \\
\hline $\mathrm{C}$ & -3.806244 & -1.849931 & 1.360602 \\
\hline $\mathrm{C}$ & -1.469545 & -2.414478 & 1.135328 \\
\hline $\mathrm{C}$ & -1.732696 & -3.696017 & 1.608631 \\
\hline $\mathrm{C}$ & -4.066652 & -3.131097 & 1.833110 \\
\hline $\mathrm{H}$ & -4.618961 & -1.126047 & 1.291235 \\
\hline $\mathrm{H}$ & -0.443279 & -2.126093 & 0.891449 \\
\hline $\mathrm{H}$ & -0.917877 & -4.409231 & 1.716939 \\
\hline $\mathrm{H}$ & -5.080951 & -3.406360 & 2.115076 \\
\hline $\mathrm{H}$ & -3.239632 & -5.057709 & 2.323100 \\
\hline $\mathrm{C}$ & -2.888835 & 1.370146 & 1.471753 \\
\hline $\mathrm{C}$ & -3.990661 & 3.270169 & 3.204590 \\
\hline $\mathrm{C}$ & -3.277486 & 1.007823 & 2.765274 \\
\hline $\mathrm{C}$ & -3.039963 & 2.700008 & 1.060835 \\
\hline $\mathrm{C}$ & -3.588100 & 3.641388 & 1.924817 \\
\hline $\mathrm{C}$ & -3.832553 & 1.952802 & 3.622919 \\
\hline $\mathrm{H}$ & -3.154731 & -0.018770 & 3.108321 \\
\hline $\mathrm{H}$ & -2.720054 & 3.007811 & 0.066602 \\
\hline $\mathrm{H}$ & -3.704787 & 4.671228 & 1.592840 \\
\hline $\mathrm{H}$ & -4.140619 & 1.654672 & 4.623691 \\
\hline $\mathrm{H}$ & -4.424349 & 4.008050 & 3.876512 \\
\hline $\mathrm{C}$ & 0.292714 & 1.604753 & -3.132897 \\
\hline $\mathrm{C}$ & 0.129163 & 4.156156 & -4.291537 \\
\hline $\mathrm{C}$ & -0.692184 & 2.521465 & -2.738813 \\
\hline $\mathrm{C}$ & 1.176829 & 1.990783 & -4.146559 \\
\hline $\mathrm{C}$ & 1.091137 & 3.251209 & -4.728166 \\
\hline $\mathrm{C}$ & -0.769106 & 3.789965 & -3.294392 \\
\hline $\mathrm{H}$ & 1.951657 & 1.298167 & -4.473766 \\
\hline $\mathrm{H}$ & 1.787644 & 3.529769 & -5.515770 \\
\hline $\mathrm{H}$ & -1.539345 & 4.472341 & -2.939206 \\
\hline $\mathrm{H}$ & 0.070985 & 5.149568 & -4.731222 \\
\hline $\mathrm{C}$ & 2.155515 & -0.544841 & -2.724933 \\
\hline $\mathrm{C}$ & 4.732628 & -1.305140 & -3.489377 \\
\hline $\mathrm{C}$ & 3.273804 & 0.112494 & -2.197781 \\
\hline $\mathrm{C}$ & 2.340670 & -1.583547 & -3.640423 \\
\hline $\mathrm{C}$ & 3.627360 & -1.964158 & -4.014204 \\
\hline $\mathrm{C}$ & 4.554157 & -0.260534 & -2.584143 \\
\hline $\mathrm{H}$ & 3.146334 & 0.934128 & -1.489487 \\
\hline $\mathrm{H}$ & 1.481459 & -2.099448 & -4.066923 \\
\hline $\mathrm{H}$ & 3.761748 & -2.778863 & -4.723023 \\
\hline $\mathrm{H}$ & 5.415732 & 0.263555 & -2.173557 \\
\hline $\mathrm{H}$ & 5.736536 & -1.603358 & -3.785150 \\
\hline $\mathrm{C}$ & 0.332247 & 0.904517 & 2.166554 \\
\hline $\mathrm{C}$ & -0.021356 & 0.122031 & 3.196117 \\
\hline $\mathrm{H}$ & -0.177967 & -0.952216 & 3.074768 \\
\hline $\mathrm{C}$ & 0.481183 & 2.393179 & 2.336253 \\
\hline $\mathrm{H}$ & 1.534276 & 2.676128 & 2.194098 \\
\hline
\end{tabular}

\begin{tabular}{|c|c|c|c|}
\hline $\mathrm{H}$ & -0.098753 & 2.926327 & 1.563786 \\
\hline C & -0.011811 & 2.824897 & 3.716011 \\
\hline $\mathrm{H}$ & -1.116622 & 2.871685 & 3.727474 \\
\hline $\mathrm{H}$ & 0.373879 & 3.815311 & 3.980734 \\
\hline O & 0.437900 & 1.935035 & 4.715060 \\
\hline C & -0.163714 & 0.681324 & 4.581405 \\
\hline $\mathrm{H}$ & 0.310093 & 0.016824 & 5.317506 \\
\hline $\mathrm{H}$ & -1.243928 & 0.728562 & 4.840016 \\
\hline O & -1.596182 & 2.177272 & -1.750337 \\
\hline O & 3.131247 & 0.839421 & 2.011703 \\
\hline B & 2.129460 & 0.075711 & 1.466254 \\
\hline O & 2.283267 & -1.274845 & 1.664960 \\
\hline C & 3.363085 & -1.408370 & 2.629522 \\
\hline C & 4.169371 & -0.095293 & 2.398281 \\
\hline $\mathrm{H}$ & 1.949060 & 0.491081 & 0.206071 \\
\hline $\mathrm{C}$ & 5.130303 & -0.191512 & 1.222283 \\
\hline $\mathrm{H}$ & 4.641240 & -0.622444 & 0.337493 \\
\hline $\mathrm{H}$ & 6.007833 & -0.803815 & 1.465801 \\
\hline $\mathrm{H}$ & 5.477097 & 0.817704 & 0.966056 \\
\hline C & 4.880004 & 0.446882 & 3.619126 \\
\hline $\mathrm{H}$ & 4.177015 & 0.703219 & 4.418535 \\
\hline $\mathrm{H}$ & 5.430792 & 1.356846 & 3.351992 \\
\hline $\mathrm{H}$ & 5.603796 & -0.284948 & 4.003360 \\
\hline C & 4.116398 & -2.684759 & 2.326954 \\
\hline $\mathrm{H}$ & 4.426717 & -2.734976 & 1.278032 \\
\hline $\mathrm{H}$ & 3.476051 & -3.551498 & 2.533086 \\
\hline $\mathrm{H}$ & 5.008488 & -2.767128 & 2.962925 \\
\hline $\mathrm{C}$ & 2.748926 & -1.473953 & 4.018313 \\
\hline $\mathrm{H}$ & 2.276537 & -0.522656 & 4.292879 \\
\hline $\mathrm{H}$ & 3.507727 & -1.707936 & 4.775535 \\
\hline $\mathrm{H}$ & 1.989306 & -2.266510 & 4.040709 \\
\hline
\end{tabular}

SCF energy: -2953.406231450

M0 6-D3 (Gas Phase)/6-31G(d) +LANL2DZ (Pd) opt. 1 imaginary frequency: $164 \mathrm{i} \mathrm{cm-1}$ 


\begin{tabular}{|c|c|c|c|}
\hline \multicolumn{4}{|l|}{ Int- 6} \\
\hline $\mathrm{Pd}$ & 0.153665 & 0.952691 & 0.478497 \\
\hline $\mathrm{P}$ & 0.666830 & 0.680301 & -1.781933 \\
\hline $\mathrm{P}$ & -2.216805 & 0.160337 & 0.527883 \\
\hline $\mathrm{C}$ & -0.030971 & -0.880908 & -2.396101 \\
\hline $\mathrm{C}$ & -0.974734 & -3.365165 & -3.221743 \\
\hline $\mathrm{C}$ & -0.540184 & -1.035100 & -3.685724 \\
\hline $\mathrm{C}$ & 0.021195 & -1.976871 & -1.529528 \\
\hline $\mathrm{C}$ & -0.447712 & -3.217459 & -1.940610 \\
\hline $\mathrm{C}$ & -1.019399 & -2.277870 & -4.090548 \\
\hline $\mathrm{H}$ & -0.569917 & -0.185981 & -4.369404 \\
\hline $\mathrm{H}$ & 0.420946 & -1.846775 & -0.521219 \\
\hline $\mathrm{H}$ & -0.413218 & -4.063045 & -1.254294 \\
\hline $\mathrm{H}$ & -1.432534 & -2.395585 & -5.090672 \\
\hline $\mathrm{H}$ & -1.354207 & -4.332481 & -3.545336 \\
\hline $\mathrm{C}$ & -3.006952 & 0.012007 & -1.128879 \\
\hline $\mathrm{C}$ & -4.077758 & -0.179677 & -3.719881 \\
\hline $\mathrm{C}$ & -2.780552 & 1.006020 & -2.087229 \\
\hline $\mathrm{C}$ & -3.758090 & -1.100712 & -1.520775 \\
\hline $\mathrm{C}$ & -4.289131 & -1.200809 & -2.801525 \\
\hline $\mathrm{C}$ & -3.317816 & 0.928419 & -3.366334 \\
\hline $\mathrm{H}$ & -3.913305 & -1.918123 & -0.820580 \\
\hline $\mathrm{H}$ & -4.867830 & -2.079455 & -3.077706 \\
\hline $\mathrm{H}$ & -3.131125 & 1.725787 & -4.082835 \\
\hline $\mathrm{H}$ & -4.499009 & -0.243223 & -4.721293 \\
\hline $\mathrm{C}$ & -2.550951 & -1.452009 & 1.322777 \\
\hline $\mathrm{C}$ & -3.006929 & -3.935468 & 2.524987 \\
\hline $\mathrm{C}$ & -3.858540 & -1.877461 & 1.601897 \\
\hline $\mathrm{C}$ & -1.479274 & -2.276941 & 1.669071 \\
\hline $\mathrm{C}$ & -1.708277 & -3.513547 & 2.268162 \\
\hline $\mathrm{C}$ & -4.083180 & -3.113194 & 2.194172 \\
\hline $\mathrm{H}$ & -4.704556 & -1.232524 & 1.361633 \\
\hline $\mathrm{H}$ & -0.456125 & -1.941194 & 1.504008 \\
\hline $\mathrm{H}$ & -0.863776 & -4.144268 & 2.545270 \\
\hline $\mathrm{H}$ & -5.101421 & -3.434231 & 2.404926 \\
\hline $\mathrm{H}$ & -3.185720 & -4.901199 & 2.994056 \\
\hline $\mathrm{C}$ & -3.294958 & 1.313744 & 1.469873 \\
\hline $\mathrm{C}$ & -4.729144 & 3.175115 & 2.995848 \\
\hline $\mathrm{C}$ & -3.591039 & 1.056898 & 2.814234 \\
\hline $\mathrm{C}$ & -3.722513 & 2.519181 & 0.901457 \\
\hline $\mathrm{C}$ & -4.436082 & 3.439746 & 1.661476 \\
\hline $\mathrm{C}$ & -4.307546 & 1.980077 & 3.568842 \\
\hline $\mathrm{H}$ & -3.269440 & 0.126076 & 3.280661 \\
\hline $\mathrm{H}$ & -3.488645 & 2.752107 & -0.134885 \\
\hline $\mathrm{H}$ & -4.765083 & 4.371515 & 1.205251 \\
\hline $\mathrm{H}$ & -4.535900 & 1.761087 & 4.610403 \\
\hline $\mathrm{H}$ & -5.286456 & 3.898614 & 3.587368 \\
\hline $\mathrm{C}$ & 0.097268 & 2.048335 & -2.851444 \\
\hline $\mathrm{C}$ & -0.710639 & 4.322914 & -4.279043 \\
\hline $\mathrm{C}$ & -1.159261 & 2.630939 & -2.637452 \\
\hline $\mathrm{C}$ & 0.929114 & 2.616883 & -3.822974 \\
\hline $\mathrm{C}$ & 0.524488 & 3.738371 & -4.538626 \\
\hline $\mathrm{C}$ & -1.558122 & 3.767131 & -3.325985 \\
\hline $\mathrm{H}$ & 1.910328 & 2.184431 & -4.013006 \\
\hline $\mathrm{H}$ & 1.184422 & 4.163609 & -5.291326 \\
\hline $\mathrm{H}$ & -2.534497 & 4.196630 & -3.108456 \\
\hline $\mathrm{H}$ & -1.021413 & 5.212232 & -4.823228 \\
\hline $\mathrm{C}$ & 2.449955 & 0.549907 & -2.179056 \\
\hline $\mathrm{C}$ & 5.160951 & 0.443888 & -2.842881 \\
\hline $\mathrm{C}$ & 3.336974 & 1.516780 & -1.691974 \\
\hline $\mathrm{C}$ & 2.932864 & -0.468705 & -3.003780 \\
\hline $\mathrm{C}$ & 4.287650 & -0.523588 & -3.325106 \\
\hline $\mathrm{C}$ & 4.682208 & 1.470291 & -2.029411 \\
\hline $\mathrm{H}$ & 2.970579 & 2.319187 & -1.047852 \\
\hline $\mathrm{H}$ & 2.257138 & -1.228291 & -3.394313 \\
\hline $\mathrm{H}$ & 4.656854 & -1.327124 & -3.959286 \\
\hline $\mathrm{H}$ & 5.361498 & 2.231053 & -1.650073 \\
\hline $\mathrm{H}$ & 6.218055 & 0.400052 & -3.097594 \\
\hline $\mathrm{C}$ & 1.353122 & 1.219606 & 2.549625 \\
\hline $\mathrm{C}$ & 0.000434 & 1.271446 & 2.822933 \\
\hline $\mathrm{H}$ & -0.542537 & 0.343610 & 3.017031 \\
\hline $\mathrm{C}$ & 2.159501 & 2.493952 & 2.655958 \\
\hline $\mathrm{H}$ & 2.655518 & 2.483828 & 3.640000 \\
\hline
\end{tabular}

\begin{tabular}{|c|c|c|c|}
\hline $\mathrm{H}$ & 2.963493 & 2.520757 & 1.908255 \\
\hline C & 1.263717 & 3.709783 & 2.566233 \\
\hline $\mathrm{H}$ & 0.851866 & 3.806721 & 1.539683 \\
\hline $\mathrm{H}$ & 1.803200 & 4.632956 & 2.803892 \\
\hline 0 & 0.219613 & 3.598786 & 3.504884 \\
\hline C & -0.677728 & 2.567520 & 3.196072 \\
\hline $\mathrm{H}$ & -1.297068 & 2.414472 & 4.089954 \\
\hline $\mathrm{H}$ & -1.368040 & 2.885074 & 2.388848 \\
\hline 0 & -2.006220 & 2.085053 & -1.697194 \\
\hline 0 & 3.488710 & -0.177046 & 2.372746 \\
\hline B & 2.129109 & -0.125410 & 2.360730 \\
\hline 0 & 1.534114 & -1.343995 & 2.157906 \\
\hline $\mathrm{C}$ & 2.585506 & -2.339579 & 2.254233 \\
\hline C & 3.861603 & -1.504391 & 1.924258 \\
\hline $\mathrm{H}$ & 1.612230 & 1.542723 & 0.299012 \\
\hline C & 4.132382 & -1.390931 & 0.433591 \\
\hline $\mathrm{H}$ & 3.232506 & -1.082950 & -0.118417 \\
\hline $\mathrm{H}$ & 4.494806 & -2.336274 & 0.009913 \\
\hline $\mathrm{H}$ & 4.899934 & -0.624781 & 0.269109 \\
\hline $\mathrm{C}$ & 5.111856 & -1.937809 & 2.657280 \\
\hline $\mathrm{H}$ & 4.993268 & -1.871630 & 3.743668 \\
\hline $\mathrm{H}$ & 5.951266 & -1.293580 & 2.369684 \\
\hline $\mathrm{H}$ & 5.374334 & -2.971959 & 2.394870 \\
\hline C & 2.287866 & -3.458130 & 1.277380 \\
\hline $\mathrm{H}$ & 2.189687 & -3.094676 & 0.247599 \\
\hline $\mathrm{H}$ & 1. 348427 & -3.956363 & 1.555302 \\
\hline $\mathrm{H}$ & 3.084235 & -4.214302 & 1.300438 \\
\hline $\mathrm{C}$ & 2.551696 & -2.862771 & 3.680752 \\
\hline $\mathrm{H}$ & 2.768697 & -2.068293 & 4.406105 \\
\hline $\mathrm{H}$ & 3.270484 & -3.677509 & 3.832157 \\
\hline $\mathrm{H}$ & 1.546173 & -3.247283 & 3.894663 \\
\hline
\end{tabular}

SCF energy: -2953.449461020

M06-D3 (Gas Phase) /6-31G(d)+LANL2DZ (Pd) opt. No imaginary frequency 


\begin{tabular}{|c|c|c|c|}
\hline \multicolumn{4}{|l|}{ TS-3 } \\
\hline $\mathrm{Pd}$ & 0.468725 & 0.735933 & -0.961673 \\
\hline $\mathrm{P}$ & 0.850947 & 0.255990 & -3.226556 \\
\hline $\mathrm{P}$ & -1.929989 & -0.024612 & -0.827271 \\
\hline $\mathrm{C}$ & 0.196410 & -1.397973 & -3.624040 \\
\hline $\mathrm{C}$ & -0.694266 & -3.996457 & -4.096664 \\
\hline $\mathrm{C}$ & -0.305164 & -1.745239 & -4.878803 \\
\hline $\mathrm{C}$ & 0.258965 & -2.361700 & -2.611991 \\
\hline $\mathrm{C}$ & -0.181302 & -3.657819 & -2.846561 \\
\hline $\mathrm{C}$ & -0.756397 & -3.042081 & -5.108454 \\
\hline $\mathrm{H}$ & -0.350131 & -0.999888 & -5.674103 \\
\hline $\mathrm{H}$ & 0.642901 & -2.079775 & -1.628249 \\
\hline $\mathrm{H}$ & -0.133906 & -4.397861 & -2.048427 \\
\hline $\mathrm{H}$ & -1.160650 & -3.307158 & -6.083841 \\
\hline $\mathrm{H}$ & -1.050143 & -5.008025 & -4.283334 \\
\hline $\mathrm{C}$ & -2.793968 & -0.449815 & -2.398187 \\
\hline $\mathrm{C}$ & -3.913375 & -1.051732 & -4.904844 \\
\hline $\mathrm{C}$ & -2.638925 & 0.409602 & -3.492361 \\
\hline $\mathrm{C}$ & -3.490437 & -1.643242 & -2.611532 \\
\hline $\mathrm{C}$ & -4.046684 & -1.945699 & -3.849617 \\
\hline $\mathrm{C}$ & -3.201806 & 0.129039 & -4.731354 \\
\hline $\mathrm{H}$ & -3.578728 & -2.367859 & -1.805718 \\
\hline $\mathrm{H}$ & -4.580346 & -2.883856 & -3.985846 \\
\hline $\mathrm{H}$ & -3.068629 & 0.825341 & -5.556808 \\
\hline $\mathrm{H}$ & -4.353742 & -1.274839 & -5.874781 \\
\hline $\mathrm{C}$ & -2.341314 & -1.445051 & 0.265260 \\
\hline $\mathrm{C}$ & -2.782856 & -3.577867 & 2.038940 \\
\hline $\mathrm{C}$ & -3.644987 & -1.864935 & 0.571193 \\
\hline $\mathrm{C}$ & -1.268950 & -2.139983 & 0.833307 \\
\hline $\mathrm{C}$ & -1.485672 & -3.189781 & 1.722744 \\
\hline $\mathrm{C}$ & -3.862122 & -2.919136 & 1.452087 \\
\hline $\mathrm{H}$ & -4.501725 & -1.378790 & 0.104692 \\
\hline $\mathrm{H}$ & -0.245431 & -1.845485 & 0.604005 \\
\hline $\mathrm{H}$ & -0.629921 & -3.700061 & 2.165824 \\
\hline $\mathrm{H}$ & -4.880287 & -3.231070 & 1.677522 \\
\hline $\mathrm{H}$ & -2.958330 & -4.399425 & 2.731357 \\
\hline $\mathrm{C}$ & -2.831541 & 1.428753 & -0.129961 \\
\hline $\mathrm{C}$ & -3.936024 & 3.762245 & 0.970248 \\
\hline $\mathrm{C}$ & -3.872280 & 1.354375 & 0.799580 \\
\hline $\mathrm{C}$ & -2.337510 & 2.692382 & -0.481523 \\
\hline $\mathrm{C}$ & -2.888050 & 3.847680 & 0.058226 \\
\hline $\mathrm{C}$ & -4.422399 & 2.513768 & 1.342303 \\
\hline $\mathrm{H}$ & -4.251211 & 0.392674 & 1.135850 \\
\hline $\mathrm{H}$ & -1.497136 & 2.767537 & -1.172011 \\
\hline $\mathrm{H}$ & -2.483604 & 4.818311 & -0.222122 \\
\hline $\mathrm{H}$ & -5.234496 & 2.435450 & 2.063582 \\
\hline $\mathrm{H}$ & -4.363033 & 4.666413 & 1.399776 \\
\hline $\mathrm{C}$ & 0.187964 & 1.431885 & -4.469071 \\
\hline $\mathrm{C}$ & -0.752002 & 3.455046 & -6.177191 \\
\hline $\mathrm{C}$ & -1.088267 & 1.989537 & -4.305565 \\
\hline $\mathrm{C}$ & 0.968234 & 1.893922 & -5.535178 \\
\hline $\mathrm{C}$ & 0.500263 & 2.888110 & -6.387858 \\
\hline $\mathrm{C}$ & -1.551543 & 3.003036 & -5.132463 \\
\hline $\mathrm{H}$ & 1.962582 & 1.478553 & -5.692348 \\
\hline $\mathrm{H}$ & 1.124537 & 3.228733 & -7.211005 \\
\hline $\mathrm{H}$ & -2.540714 & 3.417674 & -4.945344 \\
\hline $\mathrm{H}$ & -1.113227 & 4.247194 & -6.829755 \\
\hline $\mathrm{C}$ & 2.621414 & 0.138178 & -3.683663 \\
\hline $\mathrm{C}$ & 5.326987 & 0.043474 & -4.372520 \\
\hline C & 3.471550 & 1.202693 & -3.363179 \\
\hline $\mathrm{C}$ & 3.138087 & -0.972085 & -4.354140 \\
\hline $\mathrm{C}$ & 4.490180 & -1.020084 & -4.688261 \\
\hline $\mathrm{C}$ & 4.813602 & 1.160594 & -3.714578 \\
\hline $\mathrm{H}$ & 3.077449 & 2.068806 & -2.828908 \\
\hline $\mathrm{H}$ & 2.490233 & -1.808424 & -4.613789 \\
\hline $\mathrm{H}$ & 4.886123 & -1.894421 & -5.201423 \\
\hline $\mathrm{H}$ & 5.464372 & 1.996452 & -3.464836 \\
\hline $\mathrm{H}$ & 6.382116 & 0.004509 & -4.636726 \\
\hline $\mathrm{C}$ & 1.048594 & 1.248341 & 1.171309 \\
\hline $\mathrm{C}$ & -0.226657 & 1.074543 & 1.766572 \\
\hline $\mathrm{H}$ & -0.700200 & 0.092066 & 1.743155 \\
\hline $\mathrm{C}$ & 1.567346 & 2.676990 & 1.306770 \\
\hline $\mathrm{H}$ & 2.662642 & 2.703117 & 1.223444 \\
\hline
\end{tabular}

\begin{tabular}{|c|c|c|c|}
\hline $\mathrm{H}$ & 1.169304 & 3.327038 & 0.514053 \\
\hline C & 1.116162 & 3.255608 & 2.639245 \\
\hline $\mathrm{H}$ & 1.449878 & 4.288825 & 2.782095 \\
\hline $\mathrm{H}$ & 1.513950 & 2.644642 & 3.475033 \\
\hline O & -0.301324 & 3.288166 & 2.721580 \\
\hline C & -0.867801 & 2.036084 & 2.546767 \\
\hline $\mathrm{H}$ & -0.758670 & 1.197951 & 3.742415 \\
\hline $\mathrm{H}$ & -1.961940 & 2.091998 & 2.570507 \\
\hline O & -1.894902 & 1.550111 & -3.277396 \\
\hline O & 3.408070 & 0.199808 & 1.061555 \\
\hline B & 2.047390 & 0.067861 & 1.174570 \\
\hline O & 1.665385 & -1.255629 & 1.347212 \\
\hline C & 2.869841 & -2.039991 & 1.469292 \\
\hline C & 3.939802 & -1.115920 & 0.808784 \\
\hline C & 4.017566 & -1.282678 & -0.701665 \\
\hline $\mathrm{H}$ & 3.023353 & -1.222120 & -1.166418 \\
\hline $\mathrm{H}$ & 4.480890 & -2.236005 & -0.987304 \\
\hline $\mathrm{H}$ & 4.620373 & -0.466830 & -1.120893 \\
\hline C & 5.324395 & -1.212771 & 1. 413261 \\
\hline $\mathrm{H}$ & 5.328181 & -0.938610 & 2.473550 \\
\hline $\mathrm{H}$ & 6.003208 & -0.530330 & 0.887713 \\
\hline $\mathrm{H}$ & 5.724119 & -2.231302 & 1.311042 \\
\hline C & 2.653890 & -3.369837 & 0.775101 \\
\hline $\mathrm{H}$ & 2.336870 & -3.241395 & -0.266859 \\
\hline $\mathrm{H}$ & 1.876153 & -3.942022 & 1.299147 \\
\hline $\mathrm{H}$ & 3.573121 & -3.971415 & 0.784312 \\
\hline $\mathrm{C}$ & 3.102103 & -2.259686 & 2.956486 \\
\hline $\mathrm{H}$ & 3.266175 & -1.307025 & 3.477983 \\
\hline $\mathrm{H}$ & 3.964961 & -2.910531 & 3.145655 \\
\hline $\mathrm{H}$ & 2.213284 & -2.741892 & 3.387073 \\
\hline $\mathrm{H}$ & 1.915924 & 1.364672 & -1.084849 \\
\hline $\mathrm{N}$ & -0.867719 & 0.372257 & 4.734693 \\
\hline C & 0.314139 & -0.499566 & 4.623421 \\
\hline $\mathrm{H}$ & 0.384168 & -0.895610 & 3.600310 \\
\hline $\mathrm{H}$ & 0.256592 & -1.334264 & 5.335162 \\
\hline $\mathrm{H}$ & 1.209030 & 0.096477 & 4.836011 \\
\hline C & -2.121061 & -0.341260 & 4.431706 \\
\hline $\mathrm{H}$ & -2.955161 & 0.365270 & 4.505070 \\
\hline $\mathrm{H}$ & -2.286041 & -1.175566 & 5.125712 \\
\hline $\mathrm{C}$ & -0.910296 & 1.124308 & 5.982370 \\
\hline C & -0.965907 & 2.582308 & 8.337252 \\
\hline C & -0.743936 & 2.504619 & 5.933733 \\
\hline C & -1.102428 & 0.464830 & 7.193496 \\
\hline $\mathrm{C}$ & -1.130224 & 1.199994 & 8.372630 \\
\hline C & -0.773638 & 3.229891 & 7.121899 \\
\hline $\mathrm{H}$ & -0.600202 & 3.012636 & 4.978418 \\
\hline $\mathrm{H}$ & -1.227217 & -0.617668 & 7.221448 \\
\hline $\mathrm{H}$ & -1.279686 & 0.690509 & 9.322331 \\
\hline $\mathrm{H}$ & -0.645871 & 4.309968 & 7.092216 \\
\hline $\mathrm{H}$ & -0.987934 & 3.155137 & 9.262020 \\
\hline $\mathrm{H}$ & -2.068468 & -0.737767 & 3.407745 \\
\hline
\end{tabular}

\footnotetext{
SCF energy: -3319.345804370

M06-D3 (Gas Phase) /6-31G(d)+LANL2DZ (Pd) opt. 1 imaginary frequency: $748 \mathrm{i} \mathrm{cm-1}$
} 


\begin{tabular}{|c|c|c|c|}
\hline \multicolumn{4}{|l|}{ TS-4 } \\
\hline $\mathrm{Pd}$ & 0.901647 & 1.249016 & 1.195876 \\
\hline $\mathrm{P}$ & 1.721990 & 0.987593 & -1.356454 \\
\hline $\mathrm{P}$ & -1.451693 & 0.970302 & 0.905142 \\
\hline $\mathrm{C}$ & 0.770947 & -0.387083 & -2.093432 \\
\hline $\mathrm{C}$ & -0.549682 & -2.639711 & -3.081184 \\
\hline $\mathrm{C}$ & 0.239763 & -0.377238 & -3.382991 \\
\hline $\mathrm{C}$ & 0.658053 & -1.541996 & -1.312837 \\
\hline $\mathrm{C}$ & 0.007072 & -2.667133 & -1.805120 \\
\hline $\mathrm{C}$ & -0.431091 & -1.496603 & -3.867358 \\
\hline $\mathrm{H}$ & 0.345750 & 0.509405 & -4.008895 \\
\hline $\mathrm{H}$ & 1.089458 & -1.554226 & -0.307471 \\
\hline $\mathrm{H}$ & -0.074309 & -3.560864 & -1.185750 \\
\hline $\mathrm{H}$ & -0.860790 & -1.476243 & -4.867568 \\
\hline $\mathrm{H}$ & -1.072693 & -3.512839 & -3.466733 \\
\hline $\mathrm{C}$ & -2.056355 & 0.942963 & -0.832561 \\
\hline $\mathrm{C}$ & -3.004913 & 1.111422 & -3.463191 \\
\hline $\mathrm{C}$ & -1.596811 & 1.935029 & -1.703949 \\
\hline $\mathrm{C}$ & -2.995987 & 0.027906 & -1.315437 \\
\hline $\mathrm{C}$ & -3.464453 & 0.104684 & -2.622721 \\
\hline $\mathrm{C}$ & -2.068977 & 2.032563 & -3.007394 \\
\hline $\mathrm{H}$ & -3.369346 & -0.759083 & -0.662504 \\
\hline $\mathrm{H}$ & -4.193024 & -0.620633 & -2.978257 \\
\hline $\mathrm{H}$ & -1.699109 & 2.818816 & -3.662364 \\
\hline $\mathrm{H}$ & -3.374534 & 1.186080 & -4.484219 \\
\hline $\mathrm{C}$ & -2.357882 & -0.474898 & 1.576822 \\
\hline $\mathrm{C}$ & -3.762371 & -2.780860 & 2.307312 \\
\hline $\mathrm{C}$ & -3.544418 & -0.376664 & 2.308396 \\
\hline $\mathrm{C}$ & -1.874407 & -1.740452 & 1.232511 \\
\hline $\mathrm{C}$ & -2.581587 & -2.884893 & 1.576225 \\
\hline $\mathrm{C}$ & -4.233476 & -1.527274 & 2.684220 \\
\hline $\mathrm{H}$ & -3.938557 & 0.601264 & 2.584013 \\
\hline $\mathrm{H}$ & -0.946305 & -1.825754 & 0.668708 \\
\hline $\mathrm{H}$ & -2.199964 & -3.862081 & 1.283174 \\
\hline $\mathrm{H}$ & -5.154221 & -1.439071 & 3.258084 \\
\hline $\mathrm{H}$ & -4.314630 & -3.676997 & 2.584623 \\
\hline $\mathrm{C}$ & -2.326965 & 2.453315 & 1.532974 \\
\hline $\mathrm{C}$ & -3.593869 & 4.775050 & 2.432815 \\
\hline $\mathrm{C}$ & -1.636624 & 3.378194 & 2.315771 \\
\hline $\mathrm{C}$ & -3.656939 & 2.715311 & 1.178877 \\
\hline $\mathrm{C}$ & -4.288505 & 3.864390 & 1.638630 \\
\hline $\mathrm{C}$ & -2.264302 & 4.536891 & 2.762228 \\
\hline $\mathrm{H}$ & -0.582376 & 3.215556 & 2.541556 \\
\hline $\mathrm{H}$ & -4.197737 & 2.023743 & 0.531605 \\
\hline $\mathrm{H}$ & -5.324076 & 4.057290 & 1.364456 \\
\hline $\mathrm{H}$ & -1.699780 & 5.258776 & 3.349585 \\
\hline $\mathrm{H}$ & -4.088842 & 5.680393 & 2.779644 \\
\hline $\mathrm{C}$ & 1.391652 & 2.492492 & -2.377798 \\
\hline $\mathrm{C}$ & 1.022275 & 5.024875 & -3.562174 \\
\hline $\mathrm{C}$ & 0.277144 & 3.290657 & -2.081488 \\
\hline $\mathrm{C}$ & 2.289529 & 2.985118 & -3.332959 \\
\hline $\mathrm{C}$ & 2.104746 & 4.230639 & -3.925122 \\
\hline $\mathrm{C}$ & 0.097558 & 4.548906 & -2.638481 \\
\hline $\mathrm{H}$ & 3.158855 & 2.390363 & -3.607647 \\
\hline $\mathrm{H}$ & 2.819035 & 4.584245 & -4.665699 \\
\hline $\mathrm{H}$ & -0.778317 & 5.127391 & -2.348664 \\
\hline $\mathrm{H}$ & 0.886751 & 6.008487 & -4.007041 \\
\hline $\mathrm{C}$ & 3.430814 & 0.562353 & -1.909257 \\
\hline $\mathrm{C}$ & 6.056130 & -0.014015 & -2.717078 \\
\hline $\mathrm{C}$ & 4.486328 & 1.426376 & -1.589982 \\
\hline $\mathrm{C}$ & 3.715390 & -0.599599 & -2.634032 \\
\hline $\mathrm{C}$ & 5.020339 & -0.887946 & -3.027124 \\
\hline $\mathrm{C}$ & 5.783446 & 1.149497 & -2.000516 \\
\hline $\mathrm{H}$ & 4.300204 & 2.339963 & -1.027179 \\
\hline $\mathrm{H}$ & 2.916769 & -1.289046 & -2.902828 \\
\hline $\mathrm{H}$ & 5.221579 & -1.796917 & -3.591090 \\
\hline $\mathrm{H}$ & 6.585924 & 1.841735 & -1.750538 \\
\hline $\mathrm{H}$ & 7.073401 & -0.234937 & -3.035223 \\
\hline $\mathrm{C}$ & 2.700650 & 2.542710 & 2.402463 \\
\hline $\mathrm{C}$ & 2.425299 & 3.201658 & 1.237702 \\
\hline $\mathrm{H}$ & 2.932020 & 2.909873 & 0.319254 \\
\hline $\mathrm{C}$ & 2.250167 & 3.199620 & 3.695917 \\
\hline $\mathrm{H}$ & 1.304418 & 2.775696 & 4.057321 \\
\hline
\end{tabular}

\begin{tabular}{|c|c|c|c|}
\hline $\mathrm{H}$ & 2.993903 & 3.019868 & 4.486275 \\
\hline C & 2.048255 & 4.685639 & 3.469273 \\
\hline $\mathrm{H}$ & 3.010487 & 5.178104 & 3.235156 \\
\hline $\mathrm{H}$ & 1.620945 & 5.178906 & 4.349349 \\
\hline O & 1.130585 & 4.885051 & 2.412936 \\
\hline C & 1.652927 & 4.481143 & 1.174905 \\
\hline $\mathrm{H}$ & 0.801948 & 4.392518 & 0.481647 \\
\hline $\mathrm{H}$ & 2.320355 & 5.264631 & 0.760648 \\
\hline O & -0.673188 & 2.823607 & -1.194515 \\
\hline 0 & 3.869506 & 0.557481 & 3.523017 \\
\hline B & 3.724229 & 1.368423 & 2.428873 \\
\hline O & 4.594806 & 1.073329 & 1.419331 \\
\hline C & 5.241403 & -0.177103 & 1.767548 \\
\hline C & 5.078364 & -0.215641 & 3.319991 \\
\hline $\mathrm{H}$ & 0.441235 & 0.944568 & 2.818174 \\
\hline C & 6.185220 & 0.519568 & 4.059520 \\
\hline $\mathrm{H}$ & 6.348949 & 1.522868 & 3.645164 \\
\hline $\mathrm{H}$ & 7.133568 & -0.030441 & 4.022132 \\
\hline $\mathrm{H}$ & 5.896465 & 0.630759 & 5.111636 \\
\hline C & 4.876367 & -1.593536 & 3.913427 \\
\hline $\mathrm{H}$ & 3.976236 & -2.082203 & 3.522815 \\
\hline $\mathrm{H}$ & 4.769310 & -1.514862 & 5.002820 \\
\hline $\mathrm{H}$ & 5.742777 & -2.236675 & 3.707342 \\
\hline C & 6.678926 & -0.144368 & 1.293110 \\
\hline $\mathrm{H}$ & 7.205958 & 0.747542 & 1.647452 \\
\hline $\mathrm{H}$ & 6.707859 & -0.145499 & 0.196841 \\
\hline $\mathrm{H}$ & 7.219167 & -1.033676 & 1.646348 \\
\hline C & 4.487194 & -1.284235 & 1.046075 \\
\hline $\mathrm{H}$ & 3.425223 & -1.297025 & 1.327365 \\
\hline $\mathrm{H}$ & 4.917957 & -2.271148 & 1.258428 \\
\hline $\mathrm{H}$ & 4.539884 & -1.111930 & -0.035995 \\
\hline $\mathrm{N}$ & 0.176330 & 0.195907 & 4.229358 \\
\hline C & 1.203019 & 0.417125 & 5.267737 \\
\hline $\mathrm{H}$ & 2.200090 & 0.209096 & 4.868940 \\
\hline $\mathrm{H}$ & 0.999902 & -0.243987 & 6.125381 \\
\hline $\mathrm{H}$ & 1.159690 & 1.455779 & 5.610619 \\
\hline C & -1.110607 & 0.715218 & 4.698720 \\
\hline $\mathrm{H}$ & -1.908677 & 0.421650 & 4.011146 \\
\hline $\mathrm{H}$ & -1.057038 & 1.809822 & 4.733519 \\
\hline $\mathrm{H}$ & -1.349396 & 0.347154 & 5.708467 \\
\hline C & 0.181812 & -1.203544 & 3.862279 \\
\hline $\mathrm{C}$ & 0.391209 & -3.903501 & 3.194385 \\
\hline C & -0.702168 & -2.125217 & 4.415092 \\
\hline C & 1.173791 & -1.634570 & 2.982052 \\
\hline C & 1.274320 & -2.977885 & 2.642318 \\
\hline C & -0.592207 & -3.471941 & 4.077436 \\
\hline $\mathrm{H}$ & -1.486268 & -1.805693 & 5.099115 \\
\hline $\mathrm{H}$ & 1.873254 & -0.895761 & 2.585695 \\
\hline $\mathrm{H}$ & 2.051722 & -3.302949 & 1.949795 \\
\hline $\mathrm{H}$ & -1.289684 & -4.187322 & 4.509928 \\
\hline $\mathrm{H}$ & 0.471605 & -4.958090 & 2.937733 \\
\hline
\end{tabular}

SCF energy: -3319.384332800

M06-D3 (Gas Phase) /6-31G(d)+LANL2DZ (Pd) opt. 1 imaginary frequency: $\quad 505 i \mathrm{~cm}-1$ 


\begin{tabular}{|c|c|c|c|}
\hline \multicolumn{4}{|c|}{ Int-10 } \\
\hline $\mathrm{Pd}$ & 0.006604 & 0.918078 & 0.744286 \\
\hline $\mathrm{P}$ & 0.703786 & 0.833594 & -1.532550 \\
\hline $\mathrm{P}$ & -2.352277 & 0.331393 & 0.629368 \\
\hline $\mathrm{C}$ & 0.182515 & -0.756551 & -2.292271 \\
\hline $\mathrm{C}$ & -0.594526 & -3.262920 & -3.259124 \\
\hline $\mathrm{C}$ & -0.117789 & -0.927908 & -3.644899 \\
\hline $\mathrm{C}$ & 0.092125 & -1.855470 & -1.431247 \\
\hline $\mathrm{C}$ & -0.292864 & -3.102475 & -1.909460 \\
\hline $\mathrm{C}$ & -0.505138 & -2.176139 & -4.124481 \\
\hline $\mathrm{H}$ & -0.059701 & -0.076397 & -4.325489 \\
\hline $\mathrm{H}$ & 0.308205 & -1.713381 & -0.368923 \\
\hline $\mathrm{H}$ & -0.375916 & -3.942108 & -1.218898 \\
\hline $\mathrm{H}$ & -0.749303 & -2.297388 & -5.179138 \\
\hline $\mathrm{H}$ & -0.909028 & -4.234645 & -3.637024 \\
\hline $\mathrm{C}$ & -3.027758 & -0.046358 & -1.042148 \\
\hline $\mathrm{C}$ & -3.773853 & -0.593158 & -3.694512 \\
\hline $\mathrm{C}$ & -2.748661 & 0.853337 & -2.079048 \\
\hline $\mathrm{C}$ & -3.678943 & -1.236034 & -1.377413 \\
\hline $\mathrm{C}$ & -4.049311 & -1.513095 & -2.689577 \\
\hline $\mathrm{C}$ & -3.123686 & 0.597717 & -3.393219 \\
\hline $\mathrm{H}$ & -3.880609 & -1.970129 & -0.598862 \\
\hline $\mathrm{H}$ & -4.546979 & -2.452124 & -2.924400 \\
\hline $\mathrm{H}$ & -2.890088 & 1. 320409 & -4.173175 \\
\hline $\mathrm{H}$ & -4.059126 & -0.801643 & -4.724572 \\
\hline $\mathrm{C}$ & -2.746477 & -1.206510 & 1.559848 \\
\hline $\mathrm{C}$ & -3.231256 & -3.574758 & 2.969467 \\
\hline $\mathrm{C}$ & -4.050076 & -1.556993 & 1.931813 \\
\hline $\mathrm{C}$ & -1.690338 & -2.051539 & 1.910897 \\
\hline $\mathrm{C}$ & -1.932616 & -3.229691 & 2.612306 \\
\hline $\mathrm{C}$ & -4.290730 & -2.735288 & 2.628909 \\
\hline $\mathrm{H}$ & -4.880550 & -0.898080 & 1.673079 \\
\hline $\mathrm{H}$ & -0.664607 & -1.770188 & 1.665830 \\
\hline $\mathrm{H}$ & -1.094931 & -3.868172 & 2.894122 \\
\hline $\mathrm{H}$ & -5.308719 & -2.998382 & 2.913003 \\
\hline $\mathrm{H}$ & -3.421599 & -4.493288 & 3.522949 \\
\hline $\mathrm{C}$ & -3.597593 & 1.490200 & 1. 326023 \\
\hline $\mathrm{C}$ & -5.345517 & 3.330101 & 2.513205 \\
\hline $\mathrm{C}$ & -3.566059 & 1.718128 & 2.707641 \\
\hline $\mathrm{C}$ & -4.520075 & 2.193388 & 0.549741 \\
\hline $\mathrm{C}$ & -5.386415 & 3.109187 & 1.141130 \\
\hline $\mathrm{C}$ & -4.434136 & 2.626851 & 3.297846 \\
\hline $\mathrm{H}$ & -2.862514 & 1.161117 & 3.329440 \\
\hline $\mathrm{H}$ & -4.569809 & 2.020814 & -0.524678 \\
\hline $\mathrm{H}$ & -6.102112 & 3.648900 & 0.522342 \\
\hline $\mathrm{H}$ & -4.397504 & 2.788490 & 4.374179 \\
\hline $\mathrm{H}$ & -6.023729 & 4.047283 & 2.972848 \\
\hline $\mathrm{C}$ & 0.084457 & 2.124197 & -2.708445 \\
\hline C & -0.837209 & 4.281186 & -4.277657 \\
\hline $\mathrm{C}$ & -1.236097 & 2.589890 & -2.615831 \\
\hline $\mathrm{C}$ & 0.920676 & 2.764808 & -3.630710 \\
\hline $\mathrm{C}$ & 0.468833 & 3.824370 & -4.411246 \\
\hline $\mathrm{C}$ & -1.692773 & 3.659039 & -3.375760 \\
\hline $\mathrm{H}$ & 1.953787 & 2.434682 & -3.731674 \\
\hline $\mathrm{H}$ & 1.146794 & 4.299069 & -5.118284 \\
\hline $\mathrm{H}$ & -2.722878 & 3.985592 & -3.241826 \\
\hline $\mathrm{H}$ & -1.193440 & 5.119251 & -4.873971 \\
\hline $\mathrm{C}$ & 2.501791 & 0.869305 & -1.922735 \\
\hline $\mathrm{C}$ & 5.260267 & 1.049524 & -2.351811 \\
\hline $\mathrm{C}$ & 3.274958 & 1.788635 & -1.208733 \\
\hline $\mathrm{C}$ & 3.125009 & 0.043615 & -2.861188 \\
\hline $\mathrm{C}$ & 4.498563 & 0.133293 & -3.071658 \\
\hline $\mathrm{C}$ & 4.644804 & 1.883279 & -1.420808 \\
\hline $\mathrm{H}$ & 2.783556 & 2.436302 & -0.480648 \\
\hline $\mathrm{H}$ & 2.537518 & -0.683482 & -3.422316 \\
\hline $\mathrm{H}$ & 4.976254 & -0.520217 & -3.800423 \\
\hline $\mathrm{H}$ & 5.233510 & 2.600815 & -0.851260 \\
\hline $\mathrm{H}$ & 6.335145 & 1.112615 & -2.514657 \\
\hline $\mathrm{C}$ & 1.576549 & 1.014903 & 2.409626 \\
\hline $\mathrm{C}$ & 0.287980 & 1.098403 & 2.945393 \\
\hline $\mathrm{H}$ & -0.209629 & 0.188575 & 3.292320 \\
\hline $\mathrm{C}$ & 2.439727 & 2.256081 & 2.418038 \\
\hline $\mathrm{H}$ & 3.118698 & 2.217268 & 3.287387 \\
\hline
\end{tabular}

\begin{tabular}{rrrr} 
H & 3.089554 & 2.296182 & 1.532379 \\
$\mathrm{C}$ & 1.583811 & 3.498653 & 2.532077 \\
$\mathrm{H}$ & 0.979101 & 3.629005 & 1.609422 \\
$\mathrm{H}$ & 2.187477 & 4.403720 & 2.676621 \\
$\mathrm{O}$ & 0.741334 & 3.399195 & 3.658931 \\
$\mathrm{C}$ & -0.239977 & 2.400885 & 3.508087 \\
$\mathrm{H}$ & -0.656964 & 2.236583 & 4.513477 \\
$\mathrm{H}$ & -1.068664 & 2.788131 & 2.880657 \\
$\mathrm{O}$ & -2.111030 & 2.017865 & -1.718608 \\
$\mathrm{O}$ & 3.557620 & -0.498026 & 1.811298 \\
$\mathrm{~B}$ & 2.218723 & -0.346012 & 2.091880 \\
$\mathrm{O}$ & 1.552923 & -1.563606 & 2.110396 \\
$\mathrm{C}$ & 2.552163 & -2.594240 & 2.077127 \\
$\mathrm{C}$ & 3.767905 & -1.865635 & 1.422861 \\
$\mathrm{C}$ & 3.746593 & -1.910912 & -0.096798 \\
$\mathrm{H}$ & 2.772477 & -1.600058 & -0.498400 \\
$\mathrm{H}$ & 3.976234 & -2.914360 & -0.479941 \\
$\mathrm{H}$ & 4.498644 & -1.210977 & -0.482966 \\
$\mathrm{C}$ & 5.123843 & -2.313680 & 1.930407 \\
$\mathrm{H}$ & 5.230491 & -2.147456 & 3.007778 \\
$\mathrm{H}$ & 5.911628 & -1.743873 & 1.422013 \\
$\mathrm{H}$ & 5.286646 & -3.380506 & 1.720299 \\
$\mathrm{C}$ & 2.014203 & -3.771095 & 1.287570 \\
$\mathrm{H}$ & 1.722185 & -3.481996 & 0.271531 \\
$\mathrm{H}$ & 1.127760 & -4.179787 & 1.792414 \\
$\mathrm{H}$ & 2.762959 & -4.572866 & 1.218427 \\
$\mathrm{C}$ & 2.800851 & -3.007256 & 3.521235 \\
$\mathrm{H}$ & 3.189725 & -2.167854 & 4.112180 \\
$\mathrm{H}$ & 3.506017 & -3.845473 & 3.595651 \\
$\mathrm{H}$ & 1.846361 & -3.316450 & 3.966703 \\
------------------------------------------ \\
$\mathrm{SCF}$ & $59-10953.059496230$ & \\
\hline
\end{tabular}

SCF energy: -2953.059496230

M06-D3 (Gas Phase)/6-31G(d)+LANL2DZ (Pd) opt. No imaginary frequency 


\begin{tabular}{|c|c|c|c|}
\hline \multicolumn{4}{|l|}{ TS-5 } \\
\hline $\mathrm{Pd}$ & 0.029146 & 0.912939 & 0.512880 \\
\hline $\mathrm{P}$ & 0.597409 & 0.701077 & -1.766301 \\
\hline $\mathrm{P}$ & -2.304511 & 0.211956 & 0.508453 \\
\hline $\mathrm{C}$ & -0.023642 & -0.881067 & -2.414528 \\
\hline $\mathrm{C}$ & -0.947760 & -3.377685 & -3.233729 \\
\hline $\mathrm{C}$ & -0.534896 & -1.044404 & -3.701848 \\
\hline $\mathrm{C}$ & 0.041970 & -1.977380 & -1.547778 \\
\hline $\mathrm{C}$ & -0.416582 & -3.222894 & -1.954933 \\
\hline $\mathrm{C}$ & -1.002858 & -2.292700 & -4.104129 \\
\hline $\mathrm{H}$ & -0.579664 & -0.195953 & -4.385505 \\
\hline $\mathrm{H}$ & 0.442556 & -1.843339 & -0.539754 \\
\hline $\mathrm{H}$ & -0.374431 & -4.065940 & -1.265893 \\
\hline $\mathrm{H}$ & -1.417730 & -2.415590 & -5.103012 \\
\hline $\mathrm{H}$ & -1.321757 & -4.348405 & -3.553589 \\
\hline $\mathrm{C}$ & -3.069184 & 0.015476 & -1.151021 \\
\hline $\mathrm{C}$ & -4.078568 & -0.269253 & -3.755815 \\
\hline $\mathrm{C}$ & -2.838994 & 0.987322 & -2.131253 \\
\hline $\mathrm{C}$ & -3.787370 & -1.124443 & -1.526588 \\
\hline $\mathrm{C}$ & -4.289459 & -1.269737 & -2.814401 \\
\hline $\mathrm{C}$ & -3.347600 & 0.862984 & -3.418480 \\
\hline $\mathrm{H}$ & -3.938960 & -1.925448 & -0.806534 \\
\hline $\mathrm{H}$ & -4.843927 & -2.167385 & -3.078859 \\
\hline $\mathrm{H}$ & -3.160480 & 1.643728 & -4.153017 \\
\hline $\mathrm{H}$ & -4.477161 & -0.368880 & -4.763597 \\
\hline $\mathrm{C}$ & -2.607284 & -1.388198 & 1.334950 \\
\hline $\mathrm{C}$ & -3.008757 & -3.854050 & 2.588709 \\
\hline $\mathrm{C}$ & -3.903945 & -1.824243 & 1.643887 \\
\hline $\mathrm{C}$ & -1.518436 & -2.194371 & 1.673146 \\
\hline $\mathrm{C}$ & -1.720057 & -3.422053 & 2.298790 \\
\hline $\mathrm{C}$ & -4.101491 & -3.051911 & 2.262582 \\
\hline $\mathrm{H}$ & -4.761795 & -1.194137 & 1.405534 \\
\hline $\mathrm{H}$ & -0.502528 & -1.852624 & 1.475205 \\
\hline $\mathrm{H}$ & -0.862299 & -4.036933 & 2.570721 \\
\hline $\mathrm{H}$ & -5.111345 & -3.382491 & 2.497490 \\
\hline $\mathrm{H}$ & -3.166714 & -4.812819 & 3.079039 \\
\hline $\mathrm{C}$ & -3.368565 & 1.383100 & 1.435416 \\
\hline $\mathrm{C}$ & -4.746945 & 3.289922 & 2.952505 \\
\hline $\mathrm{C}$ & -3.622284 & 1.163175 & 2.794850 \\
\hline $\mathrm{C}$ & -3.808801 & 2.573412 & 0.846326 \\
\hline $\mathrm{C}$ & -4.495624 & 3.517073 & 1.602916 \\
\hline $\mathrm{C}$ & -4.311374 & 2.109429 & 3.545613 \\
\hline $\mathrm{H}$ & -3.285281 & 0.244775 & 3.275480 \\
\hline $\mathrm{H}$ & -3.606087 & 2.775310 & -0.203162 \\
\hline $\mathrm{H}$ & -4.836475 & 4.437204 & 1.132026 \\
\hline $\mathrm{H}$ & -4.507682 & 1.920886 & 4.599580 \\
\hline $\mathrm{H}$ & -5.282934 & 4.031720 & 3.541108 \\
\hline $\mathrm{C}$ & 0.032163 & 2.045043 & -2.872484 \\
\hline $\mathrm{C}$ & -0.769735 & 4.287070 & -4.357517 \\
\hline $\mathrm{C}$ & -1.232426 & 2.621778 & -2.692283 \\
\hline $\mathrm{C}$ & 0.876789 & 2.606403 & -3.837147 \\
\hline $\mathrm{C}$ & 0.476264 & 3.710704 & -4.581224 \\
\hline $\mathrm{C}$ & -1.629357 & 3.740676 & -3.410076 \\
\hline $\mathrm{H}$ & 1.866796 & 2.181858 & -3.998601 \\
\hline $\mathrm{H}$ & 1.147260 & 4.129260 & -5.327948 \\
\hline $\mathrm{H}$ & -2.613604 & 4.164609 & -3.218538 \\
\hline $\mathrm{H}$ & -1.078792 & 5.162772 & -4.924324 \\
\hline $\mathrm{C}$ & 2.399677 & 0.645039 & -2.082162 \\
\hline $\mathrm{C}$ & 5.147963 & 0.661695 & -2.573417 \\
\hline $\mathrm{C}$ & 3.220002 & 1.607659 & -1.483005 \\
\hline $\mathrm{C}$ & 2.966121 & -0.304935 & -2.934203 \\
\hline $\mathrm{C}$ & 4.339349 & -0.298509 & -3.171156 \\
\hline $\mathrm{C}$ & 4.585584 & 1.621039 & -1.732028 \\
\hline $\mathrm{H}$ & 2.780637 & 2.361296 & -0.824889 \\
\hline $\mathrm{H}$ & 2.338377 & -1.057214 & -3.411070 \\
\hline $\mathrm{H}$ & 4.775409 & -1.047780 & -3.829019 \\
\hline $\mathrm{H}$ & 5.214435 & 2.377086 & -1.265889 \\
\hline $\mathrm{H}$ & 6.219769 & 0.665295 & -2.762098 \\
\hline $\mathrm{C}$ & 1.444362 & 1.171973 & 2.409190 \\
\hline $\mathrm{C}$ & 0.070752 & 1.205131 & 2.730533 \\
\hline $\mathrm{H}$ & -0.409201 & 0.273477 & 3.040215 \\
\hline $\mathrm{C}$ & 2.251576 & 2.442324 & 2.592635 \\
\hline $\mathrm{H}$ & 2.714792 & 2.387585 & 3.590392 \\
\hline
\end{tabular}

\begin{tabular}{|c|c|c|c|}
\hline $\mathrm{H}$ & 3.077042 & 2.501041 & 1.870220 \\
\hline C & 1.351520 & 3.656683 & 2.534123 \\
\hline $\mathrm{H}$ & 0.934973 & 3.776641 & 1.511249 \\
\hline $\mathrm{H}$ & 1.890760 & 4.575199 & 2.789525 \\
\hline 0 & 0.315743 & 3.518985 & 3.476398 \\
\hline C & -0.593056 & 2.502016 & 3.145807 \\
\hline $\mathrm{H}$ & -1.207452 & 2.333971 & 4.039807 \\
\hline $\mathrm{H}$ & -1.285526 & 2.854073 & 2.353738 \\
\hline 0 & -2.094206 & 2.089840 & -1.755705 \\
\hline 0 & 3.568695 & -0.261084 & 2.287665 \\
\hline B & 2.212414 & -0.193306 & 2.297945 \\
\hline 0 & 1.590127 & -1.401205 & 2.141485 \\
\hline $\mathrm{C}$ & 2.627639 & -2.413549 & 2.230881 \\
\hline C & 3.910319 & -1.605924 & 1.856893 \\
\hline $\mathrm{H}$ & 1.581738 & 1.327721 & 0.688028 \\
\hline $\mathrm{C}$ & 4.149551 & -1.524701 & 0.358728 \\
\hline $\mathrm{H}$ & 3. 244618 & -1.210124 & -0.181869 \\
\hline $\mathrm{H}$ & 4.483616 & -2.485551 & -0.053010 \\
\hline $\mathrm{H}$ & 4.927205 & -0.776815 & 0.160929 \\
\hline $\mathrm{C}$ & 5.169017 & -2.044867 & 2.571256 \\
\hline $\mathrm{H}$ & 5.074982 & -1.957572 & 3.658551 \\
\hline $\mathrm{H}$ & 6.011769 & -1.419447 & 2.253619 \\
\hline $\mathrm{H}$ & 5.409677 & -3.087571 & 2.322359 \\
\hline C & 2.286583 & -3.539887 & 1.278005 \\
\hline $\mathrm{H}$ & 2.161919 & -3.186601 & 0.247664 \\
\hline $\mathrm{H}$ & 1. 349525 & -4.021801 & 1.589873 \\
\hline $\mathrm{H}$ & 3.073201 & -4.306477 & 1.286715 \\
\hline C & 2.618392 & -2.912849 & 3.665890 \\
\hline $\mathrm{H}$ & 2.866494 & -2.111099 & 4.373119 \\
\hline $\mathrm{H}$ & 3.326757 & -3.737347 & 3.813230 \\
\hline $\mathrm{H}$ & 1.612156 & -3.276884 & 3.909520 \\
\hline
\end{tabular}

SCF energy: -2953.446377590

M0 6-D3 (Gas Phase)/6-31G(d) +LANL2DZ (Pd) opt. 1 imaginary frequency: $\quad 516 i \mathrm{~cm}-1$ 


\begin{tabular}{|c|c|c|c|}
\hline \multicolumn{4}{|l|}{ Int-7 } \\
\hline $\mathrm{Pd}$ & -0.101599 & 0.932219 & 0.470708 \\
\hline $\mathrm{P}$ & 0.472435 & 0.698747 & -1.856169 \\
\hline $\mathrm{P}$ & -2.337773 & 0.269264 & 0.588881 \\
\hline $\mathrm{C}$ & -0.021694 & -0.914465 & -2.536590 \\
\hline $\mathrm{C}$ & -0.732354 & -3.465644 & -3.401246 \\
\hline $\mathrm{C}$ & -0.464909 & -1.107219 & -3.844857 \\
\hline $\mathrm{C}$ & 0.075851 & -2.006702 & -1.666784 \\
\hline $\mathrm{C}$ & -0.277355 & -3.278716 & -2.097386 \\
\hline $\mathrm{C}$ & -0.821962 & -2.383702 & -4.272221 \\
\hline $\mathrm{H}$ & -0.536128 & -0.260251 & -4.528350 \\
\hline $\mathrm{H}$ & 0.429791 & -1.848194 & -0.644102 \\
\hline $\mathrm{H}$ & -0.208283 & -4.122200 & -1.411350 \\
\hline $\mathrm{H}$ & -1.175020 & -2.532115 & -5.291153 \\
\hline $\mathrm{H}$ & -1.018457 & -4.459541 & -3.740170 \\
\hline $\mathrm{C}$ & -3.088539 & 0.000549 & -1.066040 \\
\hline $\mathrm{C}$ & -4.082310 & -0.469466 & -3.651586 \\
\hline $\mathrm{C}$ & -2.897335 & 0.928485 & -2.098465 \\
\hline $\mathrm{C}$ & -3.762235 & -1.186198 & -1.379524 \\
\hline $\mathrm{C}$ & -4.254434 & -1.424014 & -2.656399 \\
\hline $\mathrm{C}$ & -3.401413 & 0.708719 & -3.375481 \\
\hline $\mathrm{H}$ & -3.888861 & -1.950266 & -0.616244 \\
\hline $\mathrm{H}$ & -4.769146 & -2.357862 & -2.870292 \\
\hline $\mathrm{H}$ & -3.246589 & 1.456320 & -4.150954 \\
\hline $\mathrm{H}$ & -4.471536 & -0.641368 & -4.653213 \\
\hline $\mathrm{C}$ & -2.606824 & -1.311504 & 1.456945 \\
\hline $\mathrm{C}$ & -2.992860 & -3.799873 & 2.664151 \\
\hline $\mathrm{C}$ & -3.891386 & -1.717589 & 1.845496 \\
\hline $\mathrm{C}$ & -1.520336 & -2.157458 & 1.688259 \\
\hline $\mathrm{C}$ & -1.716296 & -3.399041 & 2.287262 \\
\hline $\mathrm{C}$ & -4.080448 & -2.955553 & 2.446400 \\
\hline $\mathrm{H}$ & -4.746482 & -1.062553 & 1.674315 \\
\hline $\mathrm{H}$ & -0.513152 & -1.835028 & 1.424032 \\
\hline $\mathrm{H}$ & -0.862577 & -4.053153 & 2.466146 \\
\hline $\mathrm{H}$ & -5.080189 & -3.263530 & 2.746127 \\
\hline $\mathrm{H}$ & -3.144473 & -4.769306 & 3.135042 \\
\hline $\mathrm{C}$ & -3.387212 & 1.471432 & 1.479365 \\
\hline $\mathrm{C}$ & -4.779332 & 3.414703 & 2.928219 \\
\hline $\mathrm{C}$ & -3.593471 & 1.317351 & 2.856071 \\
\hline $\mathrm{C}$ & -3.878898 & 2.612092 & 0.837440 \\
\hline $\mathrm{C}$ & -4.572296 & 3.575748 & 1.562058 \\
\hline $\mathrm{C}$ & -4.291122 & 2.282171 & 3.573447 \\
\hline $\mathrm{H}$ & -3.208333 & 0.439242 & 3.374961 \\
\hline $\mathrm{H}$ & -3.711357 & 2.759363 & -0.227190 \\
\hline $\mathrm{H}$ & -4.953911 & 4.458599 & 1.052786 \\
\hline $\mathrm{H}$ & -4.451737 & 2.147884 & 4.641478 \\
\hline $\mathrm{H}$ & -5.321993 & 4.171437 & 3.491169 \\
\hline $\mathrm{C}$ & -0.113353 & 2.002331 & -2.995096 \\
\hline C & -0.963230 & 4.203175 & -4.509847 \\
\hline $\mathrm{C}$ & -1.369812 & 2.586448 & -2.784548 \\
\hline $\mathrm{C}$ & 0.699547 & 2.535550 & -4.000842 \\
\hline $\mathrm{C}$ & 0.275609 & 3.620960 & -4.759733 \\
\hline $\mathrm{C}$ & -1.791987 & 3.684521 & -3.519765 \\
\hline $\mathrm{H}$ & 1.684087 & 2.102965 & -4.178807 \\
\hline $\mathrm{H}$ & 0.920801 & 4.020602 & -5.538956 \\
\hline $\mathrm{H}$ & -2.769570 & 4.114474 & -3.308882 \\
\hline $\mathrm{H}$ & -1.291222 & 5.062984 & -5.090241 \\
\hline $\mathrm{C}$ & 2. 291694 & 0.739670 & -2.064608 \\
\hline $\mathrm{C}$ & 5.072937 & 0.879162 & -2.291243 \\
\hline $\mathrm{C}$ & 3.005517 & 1.766729 & -1.433078 \\
\hline $\mathrm{C}$ & 2.984600 & -0.213982 & -2.813372 \\
\hline $\mathrm{C}$ & 4.372014 & -0.142403 & -2.922734 \\
\hline $\mathrm{C}$ & 4.387376 & 1.838695 & -1.547319 \\
\hline $\mathrm{H}$ & 2.469106 & 2.529879 & -0.862097 \\
\hline $\mathrm{H}$ & 2.441754 & -1.016762 & -3.312108 \\
\hline $\mathrm{H}$ & 4.905215 & -0.890546 & -3.506393 \\
\hline $\mathrm{H}$ & 4.931587 & 2.643240 & -1.056141 \\
\hline $\mathrm{H}$ & 6.156563 & 0.930863 & -2.378137 \\
\hline $\mathrm{C}$ & 1.590584 & 1.224674 & 2.144627 \\
\hline $\mathrm{C}$ & 0.145096 & 1.239218 & 2.533521 \\
\hline $\mathrm{H}$ & -0.208701 & 0.377390 & 3.108475 \\
\hline $\mathrm{C}$ & 2.450715 & 2.343506 & 2.773104 \\
\hline $\mathrm{H}$ & 2.781838 & 1.993425 & 3.761165 \\
\hline
\end{tabular}

\begin{tabular}{|c|c|c|c|}
\hline $\mathrm{H}$ & 3.356055 & 2.506875 & 2.171668 \\
\hline C & 1.653775 & 3.618026 & 2.937103 \\
\hline $\mathrm{H}$ & 1.402945 & 4.049207 & 1.944365 \\
\hline $\mathrm{H}$ & 2.219929 & 4.371808 & 3.496262 \\
\hline 0 & 0.474879 & 3.371097 & 3.663358 \\
\hline C & -0.429942 & 2.588992 & 2.919278 \\
\hline $\mathrm{H}$ & -1.330990 & 2.475036 & 3.531037 \\
\hline $\mathrm{H}$ & -0.726066 & 3.153570 & 2.008285 \\
\hline 0 & -2.200836 & 2.082812 & -1.801500 \\
\hline 0 & 3.624972 & -0.361412 & 2.295831 \\
\hline B & 2.290712 & -0.187904 & 2.113312 \\
\hline 0 & 1.618968 & -1.341972 & 1.810624 \\
\hline $\mathrm{C}$ & 2.558118 & -2.430569 & 2.021935 \\
\hline C & 3.927847 & -1.710125 & 1.846625 \\
\hline $\mathrm{H}$ & 1.643303 & 1.534078 & 1.013753 \\
\hline $\mathrm{C}$ & 4.350229 & -1.587102 & 0.392054 \\
\hline $\mathrm{H}$ & 3.533270 & -1.199935 & -0.235670 \\
\hline $\mathrm{H}$ & 4.678373 & -2.549937 & -0.019922 \\
\hline $\mathrm{H}$ & 5.186788 & -0.881382 & 0.324003 \\
\hline $\mathrm{C}$ & 5.054787 & -2.268024 & 2.686060 \\
\hline $\mathrm{H}$ & 4.834256 & -2.212369 & 3.757065 \\
\hline $\mathrm{H}$ & 5.972621 & -1.697612 & 2.500426 \\
\hline $\mathrm{H}$ & 5.249630 & -3.316433 & 2.421539 \\
\hline C & 2.272303 & -3.520344 & 1.011238 \\
\hline $\mathrm{H}$ & 2.302430 & -3.146619 & -0.019127 \\
\hline $\mathrm{H}$ & 1.272849 & -3.941397 & 1.193127 \\
\hline $\mathrm{H}$ & 2.999833 & -4.337749 & 1.105816 \\
\hline C & 2.315829 & -2.939590 & 3.433489 \\
\hline $\mathrm{H}$ & 2.536551 & -2.169952 & 4.184087 \\
\hline $\mathrm{H}$ & 2.924480 & -3.824910 & 3.655176 \\
\hline $\mathrm{H}$ & 1.257879 & -3.214750 & 3.536763 \\
\hline
\end{tabular}

SCF energy: -2953.456800880

M0 6-D3 (Gas Phase) /6-31G(d)+LANL2DZ (Pd) opt. No imaginary frequency 


\begin{tabular}{|c|c|c|c|}
\hline \multicolumn{4}{|c|}{ Int-7prime } \\
\hline $\mathrm{Pd}$ & 1.320158 & -0.901140 & -0.125600 \\
\hline $\mathrm{P}$ & 1.412776 & -0.897170 & -2.460484 \\
\hline $\mathrm{P}$ & -0.973628 & -0.265544 & 0.420413 \\
\hline $\mathrm{C}$ & -0.183159 & -1.375157 & -3.192511 \\
\hline $\mathrm{C}$ & -2.631094 & -2.179507 & -4.251079 \\
\hline $\mathrm{C}$ & -0.662651 & -0.788445 & -4.365292 \\
\hline $\mathrm{C}$ & -0.925328 & -2.380799 & -2.567306 \\
\hline $\mathrm{C}$ & -2.147402 & -2.781585 & -3.092167 \\
\hline $\mathrm{C}$ & -1.886626 & -1.191593 & -4.889508 \\
\hline $\mathrm{H}$ & -0.086854 & -0.007843 & -4.863367 \\
\hline $\mathrm{H}$ & -0.539454 & -2.842218 & -1.656972 \\
\hline $\mathrm{H}$ & -2.725362 & -3.554734 & -2.588596 \\
\hline $\mathrm{H}$ & -2.264327 & -0.724517 & -5.797054 \\
\hline $\mathrm{H}$ & -3.592710 & -2.483203 & -4.660294 \\
\hline $\mathrm{C}$ & -1.815659 & 0.663956 & -0.914775 \\
\hline $\mathrm{C}$ & -2.976116 & 2.000784 & -3.089948 \\
\hline $\mathrm{C}$ & -1.059252 & 1.516111 & -1.731744 \\
\hline $\mathrm{C}$ & -3.163665 & 0.481070 & -1.235426 \\
\hline $\mathrm{C}$ & -3.743116 & 1.140002 & -2.313873 \\
\hline $\mathrm{C}$ & -1.628840 & 2.191052 & -2.804116 \\
\hline $\mathrm{H}$ & -3.765014 & -0.204993 & -0.641261 \\
\hline $\mathrm{H}$ & -4.793968 & 0.979965 & -2.544242 \\
\hline $\mathrm{H}$ & -1.019860 & 2.852855 & -3.416679 \\
\hline $\mathrm{H}$ & -3.422795 & 2.526830 & -3.931376 \\
\hline $\mathrm{C}$ & -2.221346 & -1.421381 & 1.076774 \\
\hline $\mathrm{C}$ & -4.074992 & -3.247420 & 2.085231 \\
\hline $\mathrm{C}$ & -3.359773 & -0.968935 & 1.755888 \\
\hline $\mathrm{C}$ & -2.011705 & -2.794588 & 0.930068 \\
\hline $\mathrm{C}$ & -2.937696 & -3.705995 & 1.429346 \\
\hline $\mathrm{C}$ & -4.283584 & -1.879405 & 2.252816 \\
\hline $\mathrm{H}$ & -3.515393 & 0.101118 & 1.901294 \\
\hline $\mathrm{H}$ & -1.104351 & -3.151012 & 0.439952 \\
\hline $\mathrm{H}$ & -2.764760 & -4.774423 & 1.315104 \\
\hline $\mathrm{H}$ & -5.166565 & -1.522558 & 2.779447 \\
\hline $\mathrm{H}$ & -4.798615 & -3.958299 & 2.479597 \\
\hline $\mathrm{C}$ & -0.761265 & 0.937000 & 1.788689 \\
\hline $\mathrm{C}$ & -0.225766 & 2.665589 & 3.918470 \\
\hline $\mathrm{C}$ & -0.438034 & 0.427132 & 3.053254 \\
\hline $\mathrm{C}$ & -0.814134 & 2.319201 & 1.602744 \\
\hline $\mathrm{C}$ & -0.542107 & 3.177848 & 2.665001 \\
\hline $\mathrm{C}$ & -0.180772 & 1.286229 & 4.113671 \\
\hline $\mathrm{H}$ & -0.398578 & -0.652785 & 3.211284 \\
\hline $\mathrm{H}$ & -1.073639 & 2.732699 & 0.629292 \\
\hline $\mathrm{H}$ & -0.587940 & 4.254470 & 2.511348 \\
\hline $\mathrm{H}$ & 0.058772 & 0.879176 & 5.094214 \\
\hline $\mathrm{H}$ & -0.018758 & 3.340036 & 4.747063 \\
\hline $\mathrm{C}$ & 1.773363 & 0.792117 & -3.059794 \\
\hline $\mathrm{C}$ & 2.508787 & 3.423442 & -3.700682 \\
\hline $\mathrm{C}$ & 1.188887 & 1.889254 & -2.408275 \\
\hline $\mathrm{C}$ & 2.701584 & 1.050368 & -4.074419 \\
\hline $\mathrm{C}$ & 3.062302 & 2.354326 & -4.397677 \\
\hline $\mathrm{C}$ & 1.565942 & 3.191316 & -2.704205 \\
\hline $\mathrm{H}$ & 3.160098 & 0.217713 & -4.605818 \\
\hline $\mathrm{H}$ & 3.786884 & 2.532540 & -5.189069 \\
\hline $\mathrm{H}$ & 1.108456 & 4.007303 & -2.147301 \\
\hline $\mathrm{H}$ & 2.804854 & 4.443571 & -3.935713 \\
\hline $\mathrm{C}$ & 2.640069 & -1.983383 & -3.270696 \\
\hline $\mathrm{C}$ & 4.517270 & -3.763112 & -4.343073 \\
\hline $\mathrm{C}$ & 4.015537 & -1.758690 & -3.103698 \\
\hline $\mathrm{C}$ & 2.221303 & -3.109997 & -3.986084 \\
\hline $\mathrm{C}$ & 3.157196 & -3.991268 & -4.519065 \\
\hline $\mathrm{C}$ & 4.944941 & -2.642020 & -3.636468 \\
\hline $\mathrm{H}$ & 4.376730 & -0.882339 & -2.568134 \\
\hline $\mathrm{H}$ & 1.160119 & -3.303069 & -4.134331 \\
\hline $\mathrm{H}$ & 2.816407 & -4.860845 & -5.077531 \\
\hline $\mathrm{H}$ & 6.006536 & -2.451050 & -3.490665 \\
\hline $\mathrm{H}$ & 5.245396 & -4.457010 & -4.758292 \\
\hline 0 & 0.273964 & 1.674645 & -1.398828 \\
\hline $\mathrm{C}$ & 4.012033 & 0.458774 & -0.235344 \\
\hline $\mathrm{C}$ & 3.233322 & -1.181809 & 1.454495 \\
\hline $\mathrm{C}$ & 3.246782 & 1.089891 & 2.084061 \\
\hline 0 & 3.784862 & -0.213633 & 2.289114 \\
\hline
\end{tabular}

\begin{tabular}{|c|c|c|c|}
\hline $\mathrm{C}$ & 3.326272 & 1.537682 & 0.614576 \\
\hline C & 3.399044 & -0.897014 & -0.003080 \\
\hline $\mathrm{H}$ & 2.203498 & 1.098611 & 2.446965 \\
\hline $\mathrm{H}$ & 3.892212 & 2.473852 & 0.532519 \\
\hline $\mathrm{H}$ & 3.802158 & -1.725075 & -0.593036 \\
\hline $\mathrm{H}$ & 3.942675 & 0.748817 & -1.297868 \\
\hline $\mathrm{H}$ & 3.634464 & -2.159420 & 1.749080 \\
\hline $\mathrm{H}$ & 3.838900 & 1.736608 & 2.738370 \\
\hline $\mathrm{H}$ & 2.315253 & 1.740591 & 0.230167 \\
\hline B & 5.547866 & 0.335879 & 0.139770 \\
\hline 0 & 6.265880 & -0.799127 & -0.110489 \\
\hline C & 7.503504 & -0.656194 & 0.635038 \\
\hline $\mathrm{C}$ & 7.643642 & 0.893104 & 0.746315 \\
\hline 0 & 6.259435 & 1.329608 & 0.736935 \\
\hline C & 8.306488 & 1.524510 & -0.467465 \\
\hline $\mathrm{H}$ & 7.854108 & 1.170637 & -1.403275 \\
\hline $\mathrm{H}$ & 9.382044 & 1.309691 & -0.498780 \\
\hline $\mathrm{H}$ & 8.175894 & 2.612153 & -0.417786 \\
\hline C & 8.616358 & -1.346124 & -0.122379 \\
\hline $\mathrm{H}$ & 8.426544 & -2.425941 & -0.158056 \\
\hline $\mathrm{H}$ & 9.580813 & -1.190403 & 0.380162 \\
\hline $\mathrm{H}$ & 8.697817 & -0.981266 & -1.151955 \\
\hline C & 7.269985 & -1.334897 & 1.976379 \\
\hline $\mathrm{H}$ & 6.981803 & -2.378549 & 1.797336 \\
\hline $\mathrm{H}$ & 6.458568 & -0.849866 & 2.535937 \\
\hline $\mathrm{H}$ & 8.175504 & -1.333671 & 2.595912 \\
\hline C & 8.298832 & 1.382804 & 2.019135 \\
\hline $\mathrm{H}$ & 7.737455 & 1.077234 & 2.908227 \\
\hline $\mathrm{H}$ & 8.352049 & 2.478247 & 2.011539 \\
\hline $\mathrm{H}$ & 9.323884 & 0.995758 & 2.100417 \\
\hline $\mathrm{H}$ & 2.101363 & -1.276347 & 1.714576 \\
\hline
\end{tabular}

SCF energy: -2953.449355130

M0 6-D3 (Gas Phase) /6-31G(d)+LANL2DZ (Pd) opt. No imaginary frequency 


\begin{tabular}{|c|c|c|c|}
\hline \multicolumn{4}{|l|}{ TS- 6} \\
\hline $\mathrm{Pd}$ & -0.679031 & -0.930914 & 0.945770 \\
\hline $\mathrm{P}$ & -0.373067 & -0.902801 & -1.452229 \\
\hline $\mathrm{P}$ & -2.922306 & -0.188570 & 1.205594 \\
\hline $\mathrm{C}$ & -1.806480 & -1.392539 & -2.466375 \\
\hline $\mathrm{C}$ & -3.918223 & -2.333956 & -4.021439 \\
\hline $\mathrm{C}$ & -1.952762 & -0.956425 & -3.785803 \\
\hline $\mathrm{C}$ & -2.718049 & -2.307523 & -1.935704 \\
\hline $\mathrm{C}$ & -3.771389 & -2.779332 & -2.711128 \\
\hline $\mathrm{C}$ & -3.009850 & -1.423993 & -4.557781 \\
\hline $\mathrm{H}$ & -1.239978 & -0.246405 & -4.207756 \\
\hline $\mathrm{H}$ & -2.598679 & -2.649802 & -0.906026 \\
\hline $\mathrm{H}$ & -4.480171 & -3.488586 & -2.287432 \\
\hline $\mathrm{H}$ & -3.124593 & -1.078885 & -5.583617 \\
\hline $\mathrm{H}$ & -4.743954 & -2.698173 & -4.629869 \\
\hline $\mathrm{C}$ & -3.636224 & 0.551410 & -0.301741 \\
\hline $\mathrm{C}$ & -4.529063 & 1.631636 & -2.722962 \\
\hline $\mathrm{C}$ & -2.826096 & 1.412277 & -1.052776 \\
\hline $\mathrm{C}$ & -4.902642 & 0.236714 & -0.797401 \\
\hline $\mathrm{C}$ & -5.348510 & 0.769550 & -2.002549 \\
\hline $\mathrm{C}$ & -3.262123 & 1.959911 & -2.250907 \\
\hline $\mathrm{H}$ & -5.538882 & -0.451158 & -0.241598 \\
\hline $\mathrm{H}$ & -6.334715 & 0.507156 & -2.378728 \\
\hline $\mathrm{H}$ & -2.611033 & 2.628021 & -2.812055 \\
\hline $\mathrm{H}$ & -4.872841 & 2.052767 & -3.665664 \\
\hline $\mathrm{C}$ & -4.173761 & -1.358769 & 1.819173 \\
\hline $\mathrm{C}$ & -6.045671 & -3.214404 & 2.725969 \\
\hline $\mathrm{C}$ & -5.393189 & -0.924031 & 2.350970 \\
\hline $\mathrm{C}$ & -3.893751 & -2.726375 & 1.763507 \\
\hline $\mathrm{C}$ & -4.829639 & -3.652912 & 2.211975 \\
\hline $\mathrm{C}$ & -6.325732 & -1.851167 & 2.799299 \\
\hline $\mathrm{H}$ & -5.608188 & 0.143369 & 2.419546 \\
\hline $\mathrm{H}$ & -2.926095 & -3.060626 & 1.383588 \\
\hline $\mathrm{H}$ & -4.604843 & -4.716886 & 2.169421 \\
\hline $\mathrm{H}$ & -7.272272 & -1.510143 & 3.214097 \\
\hline $\mathrm{H}$ & -6.776954 & -3.937413 & 3.082352 \\
\hline $\mathrm{C}$ & -2.930925 & 1.186866 & 2.417388 \\
\hline $\mathrm{C}$ & -2.937495 & 3.228202 & 4.327070 \\
\hline $\mathrm{C}$ & -2.219400 & 1.041726 & 3.612825 \\
\hline $\mathrm{C}$ & -3.646312 & 2.365855 & 2.188561 \\
\hline $\mathrm{C}$ & -3.645827 & 3.381461 & 3.139807 \\
\hline $\mathrm{C}$ & -2.227494 & 2.054161 & 4.564607 \\
\hline $\mathrm{H}$ & -1.646181 & 0.132430 & 3.801465 \\
\hline $\mathrm{H}$ & -4.206848 & 2.494370 & 1.262502 \\
\hline $\mathrm{H}$ & -4.204343 & 4.296343 & 2.950770 \\
\hline $\mathrm{H}$ & -1.674700 & 1.924840 & 5.493666 \\
\hline $\mathrm{H}$ & -2.938379 & 4.024589 & 5.068882 \\
\hline $\mathrm{C}$ & 0.099096 & 0.785807 & -1.992320 \\
\hline $\mathrm{C}$ & 1.027945 & 3.403041 & -2.421308 \\
\hline $\mathrm{C}$ & -0.529211 & 1.890893 & -1.394160 \\
\hline $\mathrm{C}$ & 1.177560 & 1.035468 & -2.848285 \\
\hline $\mathrm{C}$ & 1.636976 & 2.330823 & -3.065485 \\
\hline $\mathrm{C}$ & -0.065431 & 3.184120 & -1.589202 \\
\hline $\mathrm{H}$ & 1.680811 & 0.198292 & -3.330758 \\
\hline $\mathrm{H}$ & 2.479872 & 2.499324 & -3.732671 \\
\hline $\mathrm{H}$ & -0.573171 & 4.001613 & -1.080656 \\
\hline $\mathrm{H}$ & 1.396101 & 4.415524 & -2.573129 \\
\hline $\mathrm{C}$ & 0.964384 & -1.993137 & -2.071257 \\
\hline $\mathrm{C}$ & 2.989982 & -3.791985 & -2.788359 \\
\hline $\mathrm{C}$ & 2.295553 & -1.758988 & -1.698031 \\
\hline $\mathrm{C}$ & 0.664841 & -3.141074 & -2.811313 \\
\hline $\mathrm{C}$ & 1.674308 & -4.031266 & -3.167912 \\
\hline $\mathrm{C}$ & 3.298501 & -2.650669 & -2.052433 \\
\hline $\mathrm{H}$ & 2.572837 & -0.870095 & -1.134393 \\
\hline $\mathrm{H}$ & -0.360426 & -3.347382 & -3.114902 \\
\hline $\mathrm{H}$ & 1. 425382 & -4.918085 & -3.747574 \\
\hline $\mathrm{H}$ & 4.321834 & -2.449756 & -1.738919 \\
\hline $\mathrm{H}$ & 3.774586 & -4.493582 & -3.064841 \\
\hline 0 & -1.583967 & 1.702342 & -0.526468 \\
\hline $\mathrm{C}$ & 1.947249 & 0.512671 & 1.089765 \\
\hline $\mathrm{C}$ & 0.983396 & -1.119893 & 2.744357 \\
\hline $\mathrm{C}$ & 1.624022 & 1.058748 & 3.485469 \\
\hline 0 & 1.042426 & -0.229925 & 3.750891 \\
\hline
\end{tabular}

\begin{tabular}{|c|c|c|c|}
\hline $\mathrm{C}$ & 1.372678 & 1.533326 & 2.074501 \\
\hline $\mathrm{C}$ & 1.445726 & -0.872596 & 1.421870 \\
\hline $\mathrm{H}$ & 1.189957 & 1.725899 & 4.236889 \\
\hline $\mathrm{H}$ & 1.851433 & 2.510665 & 1.933868 \\
\hline $\mathrm{H}$ & 1.864833 & -1.733888 & 0.896792 \\
\hline $\mathrm{H}$ & 1.603851 & 0.802809 & 0.078629 \\
\hline $\mathrm{H}$ & 0.973683 & -2.145491 & 3.117179 \\
\hline $\mathrm{H}$ & 2.707052 & 0.979395 & 3.679282 \\
\hline $\mathrm{H}$ & 0.290552 & 1.672173 & 1.916578 \\
\hline B & 3.513240 & 0.600223 & 0.940072 \\
\hline O & 4.300708 & -0.460571 & 0.593710 \\
\hline $\mathrm{C}$ & 5.668432 & 0.030654 & 0.602854 \\
\hline $\mathrm{C}$ & 5.463190 & 1.557237 & 0.379027 \\
\hline O & 4.180208 & 1.786897 & 1.017002 \\
\hline C & 5.275792 & 1.921584 & -1.087173 \\
\hline $\mathrm{H}$ & 4.535974 & 1.266903 & -1.572516 \\
\hline $\mathrm{H}$ & 6.216866 & 1.856865 & -1.647541 \\
\hline $\mathrm{H}$ & 4.905625 & 2.952129 & -1.151244 \\
\hline C & 6.450027 & -0.673396 & -0.484515 \\
\hline $\mathrm{H}$ & 6.534384 & -1.742034 & -0.249368 \\
\hline $\mathrm{H}$ & 7.467364 & -0.265219 & -0.556399 \\
\hline $\mathrm{H}$ & 5.968421 & -0.574357 & -1.464443 \\
\hline C & 6.241137 & -0.303919 & 1.970323 \\
\hline $\mathrm{H}$ & 6.149443 & -1.383196 & 2.141079 \\
\hline $\mathrm{H}$ & 5.698186 & 0.214419 & 2.772015 \\
\hline $\mathrm{H}$ & 7.302376 & -0.034856 & 2.040745 \\
\hline $\mathrm{C}$ & 6.504164 & 2.446311 & 1.020434 \\
\hline $\mathrm{H}$ & 6.536264 & 2.316606 & 2.107167 \\
\hline $\mathrm{H}$ & 6.274670 & 3.498277 & 0.812070 \\
\hline $\mathrm{H}$ & 7.501192 & 2.231133 & 0.612254 \\
\hline $\mathrm{H}$ & -0.624225 & -1.178679 & 2.575486 \\
\hline
\end{tabular}

SCF energy: -2953.439506470

M0 6-D3 (Gas Phase)/6-31G(d) +LANL2DZ (Pd) opt. 1 imaginary frequency: $\quad 574 i \mathrm{~cm}-1$ 


\begin{tabular}{|c|c|c|c|}
\hline \multicolumn{4}{|c|}{ Int-8 } \\
\hline $\mathrm{Pd}$ & 0.438610 & 0.008303 & 2.526432 \\
\hline $\mathrm{P}$ & 0.843840 & 0.117232 & 0.091162 \\
\hline $\mathrm{P}$ & -1.709495 & 0.848104 & 2.747853 \\
\hline $\mathrm{C}$ & -0.574430 & -0.348326 & -0.959824 \\
\hline $\mathrm{C}$ & -2.653547 & -1.263605 & -2.575597 \\
\hline $\mathrm{C}$ & -0.684828 & 0.097262 & -2.279565 \\
\hline $\mathrm{C}$ & -1.507095 & -1.259240 & -0.459272 \\
\hline $\mathrm{C}$ & -2.543106 & -1.718965 & -1.265207 \\
\hline $\mathrm{C}$ & -1.725676 & -0.355847 & -3.081431 \\
\hline $\mathrm{H}$ & 0.044226 & 0.803745 & -2.678978 \\
\hline $\mathrm{H}$ & -1.415621 & -1.611079 & 0.570499 \\
\hline $\mathrm{H}$ & -3.266531 & -2.427568 & -0.865925 \\
\hline $\mathrm{H}$ & -1.811210 & -0.002903 & -4.107488 \\
\hline $\mathrm{H}$ & -3.465276 & -1.618639 & -3.207838 \\
\hline $\mathrm{C}$ & -2.385995 & 1.581847 & 1.223353 \\
\hline $\mathrm{C}$ & -3.245283 & 2.678016 & -1.199395 \\
\hline $\mathrm{C}$ & -1.563994 & 2.446918 & 0.491077 \\
\hline $\mathrm{C}$ & -3.646340 & 1.269357 & 0.710309 \\
\hline $\mathrm{C}$ & -4.075902 & 1.812223 & -0.495967 \\
\hline $\mathrm{C}$ & -1.984288 & 3.002227 & -0.709186 \\
\hline $\mathrm{H}$ & -4.290637 & 0.578858 & 1.253114 \\
\hline $\mathrm{H}$ & -5.057091 & 1.553457 & -0.887234 \\
\hline $\mathrm{H}$ & -1.324940 & 3.672797 & -1.257510 \\
\hline $\mathrm{H}$ & -3.576115 & 3.105405 & -2.143888 \\
\hline $\mathrm{C}$ & -2.981182 & -0.307206 & 3.340156 \\
\hline $\mathrm{C}$ & -4.915403 & -2.122276 & 4.191215 \\
\hline $\mathrm{C}$ & -4.196065 & 0.154431 & 3.858791 \\
\hline $\mathrm{C}$ & -2.736807 & -1.680441 & 3.266326 \\
\hline $\mathrm{C}$ & -3.704658 & -2.586451 & 3.686772 \\
\hline $\mathrm{C}$ & -5.159290 & -0.753465 & 4.281405 \\
\hline $\mathrm{H}$ & -4.384286 & 1.225895 & 3.939393 \\
\hline $\mathrm{H}$ & -1.772042 & -2.034335 & 2.897650 \\
\hline $\mathrm{H}$ & -3.508265 & -3.655346 & 3.631106 \\
\hline $\mathrm{H}$ & -6.101299 & -0.392318 & 4.689233 \\
\hline $\mathrm{H}$ & -5.670792 & -2.830172 & 4.526954 \\
\hline $\mathrm{C}$ & -1.698276 & 2.234517 & 3.943180 \\
\hline $\mathrm{C}$ & -1.740141 & 4.307171 & 5.818513 \\
\hline $\mathrm{C}$ & -0.961912 & 2.132270 & 5.128079 \\
\hline $\mathrm{C}$ & -2.453862 & 3.388088 & 3.706347 \\
\hline $\mathrm{C}$ & -2.471762 & 4.418360 & 4.640693 \\
\hline $\mathrm{C}$ & -0.988399 & 3.161312 & 6.062052 \\
\hline $\mathrm{H}$ & -0.352928 & 1.247410 & 5.319894 \\
\hline $\mathrm{H}$ & -3.031628 & 3.485680 & 2.787240 \\
\hline $\mathrm{H}$ & -3.061868 & 5.311710 & 4.445306 \\
\hline $\mathrm{H}$ & -0.415926 & 3.066771 & 6.983253 \\
\hline $\mathrm{H}$ & -1.754330 & 5.115352 & 6.547276 \\
\hline $\mathrm{C}$ & 1.340275 & 1.805392 & -0.437453 \\
\hline C & 2.323626 & 4.412425 & -0.813956 \\
\hline $\mathrm{C}$ & 0.734953 & 2.912487 & 0.179297 \\
\hline $\mathrm{C}$ & 2.419450 & 2.051491 & -1.293492 \\
\hline $\mathrm{C}$ & 2.905212 & 3.341397 & -1.485010 \\
\hline $\mathrm{C}$ & 1.225241 & 4.199614 & 0.013099 \\
\hline $\mathrm{H}$ & 2.904347 & 1.215045 & -1.795455 \\
\hline $\mathrm{H}$ & 3.748544 & 3.506264 & -2.152811 \\
\hline $\mathrm{H}$ & 0.734248 & 5.016568 & 0.538530 \\
\hline $\mathrm{H}$ & 2.715007 & 5.419295 & -0.943434 \\
\hline $\mathrm{C}$ & 2.175952 & -0.982195 & -0.530713 \\
\hline $\mathrm{C}$ & 4.184470 & -2.805024 & -1.241077 \\
\hline $\mathrm{C}$ & 3.516019 & -0.738386 & -0.198255 \\
\hline $\mathrm{C}$ & 1.859490 & -2.153219 & -1.227279 \\
\hline $\mathrm{C}$ & 2.859653 & -3.054695 & -1.580714 \\
\hline $\mathrm{C}$ & 4.510258 & -1.642010 & -0.548578 \\
\hline $\mathrm{H}$ & 3.808925 & 0.166662 & 0.330569 \\
\hline $\mathrm{H}$ & 0.827689 & -2.367949 & -1.501618 \\
\hline $\mathrm{H}$ & 2.596502 & -3.958381 & -2.127156 \\
\hline $\mathrm{H}$ & 5.540665 & -1.431262 & -0.266413 \\
\hline $\mathrm{H}$ & 4.962106 & -3.515254 & -1.515188 \\
\hline 0 & -0.330634 & 2.728866 & 1.036195 \\
\hline $\mathrm{C}$ & 3.157533 & 1.459884 & 2.623366 \\
\hline $\mathrm{C}$ & 2.385744 & -0.266448 & 4.246305 \\
\hline $\mathrm{C}$ & 2.886534 & 1.903173 & 5.049632 \\
\hline 0 & 2.350897 & 0.582751 & 5.269452 \\
\hline
\end{tabular}

\begin{tabular}{|c|c|c|c|}
\hline $\mathrm{C}$ & 2.587115 & 2.426509 & 3.664301 \\
\hline C & 2.723879 & 0.050556 & 2.949149 \\
\hline $\mathrm{H}$ & 2.447007 & 2.520405 & 5.839139 \\
\hline $\mathrm{H}$ & 3.034634 & 3.422491 & 3.553419 \\
\hline $\mathrm{H}$ & 3.022821 & -0.780125 & 2.307915 \\
\hline $\mathrm{H}$ & 2.747823 & 1.759857 & 1.641842 \\
\hline $\mathrm{H}$ & 2.193504 & -1.291175 & 4.559739 \\
\hline $\mathrm{H}$ & 3.974465 & 1.846223 & 5.218799 \\
\hline $\mathrm{H}$ & 1.497712 & 2.539135 & 3.539144 \\
\hline B & 4.708804 & 1.618670 & 2.410303 \\
\hline 0 & 5.528952 & 0.602730 & 2.007852 \\
\hline C & 6.874056 & 1.149370 & 1.987989 \\
\hline $\mathrm{C}$ & 6.600551 & 2.672022 & 1.816405 \\
\hline 0 & 5.330546 & 2.830216 & 2.498716 \\
\hline C & 6.353881 & 3.070314 & 0.368023 \\
\hline $\mathrm{H}$ & 5.626373 & 2.399788 & -0.114166 \\
\hline $\mathrm{H}$ & 7.279238 & 3.061758 & -0.221420 \\
\hline $\mathrm{H}$ & 5.938717 & 4.085613 & 0.346293 \\
\hline C & 7.645828 & 0.508155 & 0.855456 \\
\hline $\mathrm{H}$ & 7.788749 & -0.560338 & 1.061208 \\
\hline $\mathrm{H}$ & 8.639692 & 0.965142 & 0.754624 \\
\hline $\mathrm{H}$ & 7.120928 & 0.605630 & -0.102172 \\
\hline C & 7.505304 & 0.800324 & 3.325891 \\
\hline $\mathrm{H}$ & 7.460087 & -0.285937 & 3.468538 \\
\hline $\mathrm{H}$ & 6.970005 & 1.274779 & 4.159265 \\
\hline $\mathrm{H}$ & 8.557343 & 1.108540 & 3.369452 \\
\hline C & 7.624670 & 3.584960 & 2.451830 \\
\hline $\mathrm{H}$ & 7.694049 & 3.428927 & 3.533320 \\
\hline $\mathrm{H}$ & 7.348102 & 4.632034 & 2.278779 \\
\hline $\mathrm{H}$ & 8.616900 & 3.420495 & 2.009739 \\
\hline $\mathrm{H}$ & 0.011904 & -0.251691 & 4.047061 \\
\hline
\end{tabular}

SCF energy: -2953.446117530

M06-D3 (Gas Phase) /6-31G(d)+LANL2DZ (Pd) opt. No imaginary frequency 


\begin{tabular}{|c|c|c|c|}
\hline \multicolumn{4}{|l|}{ TS-7 } \\
\hline $\mathrm{Pd}$ & -0.777565 & -0.357493 & 0.588639 \\
\hline $\mathrm{P}$ & -0.282946 & -0.430938 & -1.900226 \\
\hline $\mathrm{P}$ & -2.864534 & 0.680364 & 0.735449 \\
\hline $\mathrm{C}$ & -1.707063 & -0.852597 & -2.966253 \\
\hline $\mathrm{C}$ & -3.902914 & -1.609629 & -4.516841 \\
\hline $\mathrm{C}$ & -1.857982 & -0.355101 & -4.261904 \\
\hline $\mathrm{C}$ & -2.660245 & -1.740990 & -2.459505 \\
\hline $\mathrm{C}$ & -3.750389 & -2.125038 & -3.232382 \\
\hline $\mathrm{C}$ & -2.956933 & -0.726736 & -5.029570 \\
\hline $\mathrm{H}$ & -1.118036 & 0.335194 & -4.668515 \\
\hline $\mathrm{H}$ & -2.538263 & -2.130464 & -1.444795 \\
\hline $\mathrm{H}$ & -4.484954 & -2.822085 & -2.830533 \\
\hline $\mathrm{H}$ & -3.073719 & -0.326303 & -6.034989 \\
\hline $\mathrm{H}$ & -4.760509 & -1.899432 & -5.121023 \\
\hline $\mathrm{C}$ & -3.416634 & 1.460268 & -0.832932 \\
\hline $\mathrm{C}$ & -4.134030 & 2.587450 & -3.300440 \\
\hline $\mathrm{C}$ & -2.480444 & 2.150106 & -1.615960 \\
\hline $\mathrm{C}$ & -4.728779 & 1.379218 & -1.308758 \\
\hline $\mathrm{C}$ & -5.088324 & 1.928982 & -2.533905 \\
\hline $\mathrm{C}$ & -2.829022 & 2.710171 & -2.839237 \\
\hline $\mathrm{H}$ & -5.483305 & 0.867607 & -0.714153 \\
\hline $\mathrm{H}$ & -6.113948 & 1.842537 & -2.885509 \\
\hline $\mathrm{H}$ & -2.077826 & 3.235324 & -3.426431 \\
\hline $\mathrm{H}$ & -4.404168 & 3.019199 & -4.262185 \\
\hline $\mathrm{C}$ & -4.347714 & -0.233505 & 1.307438 \\
\hline $\mathrm{C}$ & -6.484950 & -1.797127 & 2.216744 \\
\hline $\mathrm{C}$ & -4.907316 & -0.002259 & 2.566820 \\
\hline $\mathrm{C}$ & -4.870933 & -1.258883 & 0.509427 \\
\hline $\mathrm{C}$ & -5.938150 & -2.027505 & 0.955475 \\
\hline $\mathrm{C}$ & -5.966762 & -0.786685 & 3.019613 \\
\hline $\mathrm{H}$ & -4.519014 & 0.795474 & 3.199928 \\
\hline $\mathrm{H}$ & -4.444979 & -1.447569 & -0.477443 \\
\hline $\mathrm{H}$ & -6.343177 & -2.812392 & 0.318861 \\
\hline $\mathrm{H}$ & -6.394616 & -0.596244 & 4.002235 \\
\hline $\mathrm{H}$ & -7.316985 & -2.403111 & 2.570035 \\
\hline $\mathrm{C}$ & -2.760552 & 2.090110 & 1.896674 \\
\hline $\mathrm{C}$ & -2.661596 & 4.159174 & 3.767402 \\
\hline $\mathrm{C}$ & -1.879770 & 2.020040 & 2.978655 \\
\hline $\mathrm{C}$ & -3.591910 & 3.205897 & 1.756109 \\
\hline $\mathrm{C}$ & -3.538356 & 4.236491 & 2.687722 \\
\hline $\mathrm{C}$ & -1.834399 & 3.049839 & 3.913609 \\
\hline $\mathrm{H}$ & -1.214135 & 1.161285 & 3.079169 \\
\hline $\mathrm{H}$ & -4.285762 & 3.267576 & 0.917184 \\
\hline $\mathrm{H}$ & -4.186392 & 5.103298 & 2.572072 \\
\hline $\mathrm{H}$ & -1.147668 & 2.982581 & 4.756520 \\
\hline $\mathrm{H}$ & -2.623548 & 4.967828 & 4.495056 \\
\hline $\mathrm{C}$ & 0.353626 & 1.161626 & -2.550456 \\
\hline $\mathrm{C}$ & 1.562594 & 3.650290 & -3.040997 \\
\hline $\mathrm{C}$ & -0.148571 & 2.343575 & -1.982615 \\
\hline $\mathrm{C}$ & 1.449093 & 1.270016 & -3.413505 \\
\hline $\mathrm{C}$ & 2.046785 & 2.502168 & -3.661205 \\
\hline $\mathrm{C}$ & 0.451544 & 3.574217 & -2.206596 \\
\hline $\mathrm{H}$ & 1.857891 & 0.369971 & -3.872237 \\
\hline $\mathrm{H}$ & 2.902126 & 2.562393 & -4.331668 \\
\hline $\mathrm{H}$ & 0.040064 & 4.454258 & -1.715378 \\
\hline $\mathrm{H}$ & 2.042953 & 4.611080 & -3.214307 \\
\hline $\mathrm{C}$ & 0.980203 & -1.678564 & -2.369189 \\
\hline $\mathrm{C}$ & 2.863765 & -3.731373 & -2.708707 \\
\hline $\mathrm{C}$ & 2.319688 & -1.492833 & -1.997705 \\
\hline $\mathrm{C}$ & 0.601016 & -2.909478 & -2.914877 \\
\hline $\mathrm{C}$ & 1.538474 & -3.925766 & -3.085454 \\
\hline $\mathrm{C}$ & 3.251318 & -2.509044 & -2.164714 \\
\hline $\mathrm{H}$ & 2.659268 & -0.548434 & -1.574716 \\
\hline $\mathrm{H}$ & -0.432052 & -3.079558 & -3.216318 \\
\hline $\mathrm{H}$ & 1.227644 & -4.873643 & -3.521478 \\
\hline $\mathrm{H}$ & 4.281986 & -2.337826 & -1.857028 \\
\hline $\mathrm{H}$ & 3.592618 & -4.528810 & -2.841173 \\
\hline 0 & -1.209938 & 2.281263 & -1.102299 \\
\hline $\mathrm{C}$ & 2.057672 & 1.118814 & 0.525607 \\
\hline $\mathrm{C}$ & 1.172767 & -0.302796 & 2.364257 \\
\hline $\mathrm{C}$ & 1.747376 & 1.933416 & 2.851099 \\
\hline 0 & 1.103669 & 0.711283 & 3.245794 \\
\hline
\end{tabular}

\begin{tabular}{|c|c|c|c|}
\hline $\mathrm{C}$ & 1.521254 & 2.260005 & 1.392227 \\
\hline $\mathrm{C}$ & 1.579049 & -0.203147 & 1.065066 \\
\hline $\mathrm{H}$ & 1.343267 & 2.700537 & 3.519724 \\
\hline $\mathrm{H}$ & 2.030019 & 3.202414 & 1.150034 \\
\hline $\mathrm{H}$ & 1.842367 & -1.133426 & 0.558526 \\
\hline $\mathrm{H}$ & 1.667860 & 1.271664 & -0.495173 \\
\hline $\mathrm{H}$ & 0.982312 & -1.266173 & 2.832587 \\
\hline $\mathrm{H}$ & 2.825770 & 1.824403 & 3.059495 \\
\hline $\mathrm{H}$ & 0.444353 & 2.409773 & 1.214955 \\
\hline B & 3.613848 & 1.161315 & 0.308509 \\
\hline O & 4.368503 & 0.048397 & 0.053643 \\
\hline C & 5.746666 & 0.493135 & -0.005282 \\
\hline $\mathrm{C}$ & 5.586554 & 1.997363 & -0.372705 \\
\hline 0 & 4.318956 & 2.328847 & 0.246639 \\
\hline $\mathrm{C}$ & 5.403016 & 2.224880 & -1.866230 \\
\hline $\mathrm{H}$ & 4.636324 & 1.553704 & -2.281717 \\
\hline $\mathrm{H}$ & 6.337610 & 2.072698 & -2.420654 \\
\hline $\mathrm{H}$ & 5.069515 & 3.257565 & -2.027675 \\
\hline $\mathrm{C}$ & 6.485183 & -0.339733 & -1.030676 \\
\hline $\mathrm{H}$ & 6.555958 & -1.378625 & -0.683585 \\
\hline $\mathrm{H}$ & 7.507734 & 0.035042 & -1.175059 \\
\hline $\mathrm{H}$ & 5.973555 & -0.337320 & -2.000435 \\
\hline C & 6.334404 & 0.274234 & 1.379611 \\
\hline $\mathrm{H}$ & 6.210426 & -0.779762 & 1.655771 \\
\hline $\mathrm{H}$ & 5.820867 & 0.884754 & 2.134377 \\
\hline $\mathrm{H}$ & 7.404739 & 0.514071 & 1.408276 \\
\hline C & 6.661187 & 2.909944 & 0.174542 \\
\hline $\mathrm{H}$ & 6.697682 & 2.885617 & 1.268638 \\
\hline $\mathrm{H}$ & 6.463644 & 3.943758 & -0.133761 \\
\hline $\mathrm{H}$ & 7.647699 & 2.623868 & -0.215507 \\
\hline N & -1.511907 & -2.456151 & 2.866728 \\
\hline $\mathrm{C}$ & -2.772609 & -3.095050 & 2.472597 \\
\hline $\mathrm{H}$ & -3.603086 & -2.416725 & 2.693412 \\
\hline $\mathrm{H}$ & -2.913146 & -4.037551 & 3.025023 \\
\hline $\mathrm{H}$ & -2.769106 & -3.309436 & 1.399434 \\
\hline C & -1.590642 & -1.954229 & 4.234452 \\
\hline $\mathrm{H}$ & -0.660328 & -1.443306 & 4.507897 \\
\hline $\mathrm{H}$ & -1.792900 & -2.756395 & 4.961982 \\
\hline $\mathrm{H}$ & -2.412196 & -1.228198 & 4.281662 \\
\hline $\mathrm{H}$ & -1.312908 & -1.094320 & 1.990157 \\
\hline C & -0.364226 & -3.258707 & 2.537318 \\
\hline C & 1.938749 & -4.650621 & 1.781653 \\
\hline $\mathrm{C}$ & -0.062240 & -3.448800 & 1.183607 \\
\hline C & 0.479212 & -3.793035 & 3.508823 \\
\hline C & 1.628818 & -4.483367 & 3.125187 \\
\hline C & 1.083548 & -4.134998 & 0.808415 \\
\hline $\mathrm{H}$ & -0.710821 & -3.013868 & 0.418415 \\
\hline $\mathrm{H}$ & 0.258745 & -3.667050 & 4.566737 \\
\hline $\mathrm{H}$ & 2.285482 & -4.889207 & 3.892302 \\
\hline $\mathrm{H}$ & 1.315758 & -4.248865 & -0.251106 \\
\hline $\mathrm{H}$ & 2.843124 & -5.180098 & 1.489672 \\
\hline
\end{tabular}

SCF energy: -3319.387595390

M06-D3 (Gas Phase) /6-31G(d)+LANL2DZ (Pd) opt. 1 imaginary frequency: $\quad 579 \mathrm{i} \mathrm{cm-1}$ 


\begin{tabular}{|c|c|c|c|}
\hline \multicolumn{4}{|c|}{ Int-9 } \\
\hline $\mathrm{Pd}$ & 1.010130 & 0.275805 & 0.765664 \\
\hline $\mathrm{P}$ & 1.346458 & 0.574764 & -1.569902 \\
\hline $\mathrm{P}$ & -1.361053 & 0.149955 & 1.140250 \\
\hline $\mathrm{C}$ & 0.579357 & -0.794926 & -2.532718 \\
\hline $\mathrm{C}$ & -0.597564 & -2.945969 & -3.876842 \\
\hline $\mathrm{C}$ & 0.288514 & -0.703800 & -3.897517 \\
\hline $\mathrm{C}$ & 0.284376 & -1.980123 & -1.854546 \\
\hline $\mathrm{C}$ & -0.303656 & -3.050404 & -2.521179 \\
\hline $\mathrm{C}$ & -0.295378 & -1.773747 & -4.566031 \\
\hline $\mathrm{H}$ & 0.512702 & 0.217609 & -4.437984 \\
\hline $\mathrm{H}$ & 0.502434 & -2.039766 & -0.784565 \\
\hline $\mathrm{H}$ & -0.543682 & -3.962255 & -1.976280 \\
\hline $\mathrm{H}$ & -0.523326 & -1.690164 & -5.627903 \\
\hline $\mathrm{H}$ & -1.066343 & -3.778948 & -4.398959 \\
\hline $\mathrm{C}$ & -2.370468 & -0.033414 & -0.387858 \\
\hline C & -3.512233 & -0.421030 & -2.922628 \\
\hline $\mathrm{C}$ & -2.124438 & 0.858538 & -1.443220 \\
\hline $\mathrm{C}$ & -3.215926 & -1.116356 & -0.637598 \\
\hline $\mathrm{C}$ & -3.782566 & -1.314907 & -1.893713 \\
\hline $\mathrm{C}$ & -2.685423 & 0.676186 & -2.700494 \\
\hline $\mathrm{H}$ & -3.418077 & -1.830013 & 0.160767 \\
\hline $\mathrm{H}$ & -4.430377 & -2.172374 & -2.066605 \\
\hline $\mathrm{H}$ & -2.460531 & 1.378589 & -3.501609 \\
\hline $\mathrm{H}$ & -3.940493 & -0.574814 & -3.911896 \\
\hline $\mathrm{C}$ & -2.074075 & -1.187272 & 2.185783 \\
\hline $\mathrm{C}$ & -3.088792 & -3.355729 & 3.634510 \\
\hline $\mathrm{C}$ & -3.274704 & -1.065879 & 2.894680 \\
\hline $\mathrm{C}$ & -1.382967 & -2.399220 & 2.230121 \\
\hline $\mathrm{C}$ & -1.889379 & -3.482778 & 2.941511 \\
\hline $\mathrm{C}$ & -3.776371 & -2.142498 & 3.617496 \\
\hline $\mathrm{H}$ & -3.821171 & -0.122243 & 2.875967 \\
\hline $\mathrm{H}$ & -0.428156 & -2.486346 & 1.708834 \\
\hline $\mathrm{H}$ & -1.327792 & -4.415054 & 2.973523 \\
\hline $\mathrm{H}$ & -4.711220 & -2.037481 & 4.166639 \\
\hline $\mathrm{H}$ & -3.486211 & -4.199530 & 4.198114 \\
\hline $\mathrm{C}$ & -2.067834 & 1.647682 & 1.935443 \\
\hline $\mathrm{C}$ & -3.049270 & 3.897536 & 3.277687 \\
\hline $\mathrm{C}$ & -1.355393 & 2.206497 & 3.000360 \\
\hline $\mathrm{C}$ & -3.276049 & 2.231536 & 1.548221 \\
\hline $\mathrm{C}$ & -3.761970 & 3.352697 & 2.213703 \\
\hline $\mathrm{C}$ & -1.845485 & 3.320323 & 3.672792 \\
\hline $\mathrm{H}$ & -0.401914 & 1.761769 & 3.292461 \\
\hline $\mathrm{H}$ & -3.840167 & 1.803023 & 0.718780 \\
\hline $\mathrm{H}$ & -4.703543 & 3.801877 & 1.900219 \\
\hline $\mathrm{H}$ & -1.281086 & 3.745450 & 4.501597 \\
\hline $\mathrm{H}$ & -3.430269 & 4.775465 & 3.797433 \\
\hline $\mathrm{C}$ & 0.690635 & 2.053618 & -2.461621 \\
\hline C & -0.350930 & 4.376146 & -3.666847 \\
\hline $\mathrm{C}$ & -0.580168 & 2.544626 & -2.127074 \\
\hline $\mathrm{C}$ & 1.419879 & 2.759045 & -3.424319 \\
\hline $\mathrm{C}$ & 0.907552 & 3.904594 & -4.025432 \\
\hline $\mathrm{C}$ & -1.096711 & 3.693943 & -2.711850 \\
\hline $\mathrm{H}$ & 2.412270 & 2.403958 & -3.702391 \\
\hline $\mathrm{H}$ & 1.497799 & 4.431770 & -4.773010 \\
\hline $\mathrm{H}$ & -2.081124 & 4.037856 & -2.397980 \\
\hline $\mathrm{H}$ & -0.753905 & 5.276741 & -4.126822 \\
\hline $\mathrm{C}$ & 3.092335 & 0.530888 & -2.147858 \\
\hline $\mathrm{C}$ & 5.824158 & 0.460966 & -2.760643 \\
\hline $\mathrm{C}$ & 3.985294 & 1.415810 & -1.532019 \\
\hline $\mathrm{C}$ & 3.588831 & -0.395700 & -3.068710 \\
\hline $\mathrm{C}$ & 4.948635 & -0.432863 & -3.367643 \\
\hline $\mathrm{C}$ & 5.337642 & 1.391582 & -1.844860 \\
\hline $\mathrm{H}$ & 3.611637 & 2.115955 & -0.782928 \\
\hline $\mathrm{H}$ & 2.912635 & -1.100674 & -3.551225 \\
\hline $\mathrm{H}$ & 5.322961 & -1.165202 & -4.081879 \\
\hline $\mathrm{H}$ & 6.018109 & 2.085489 & -1.353961 \\
\hline $\mathrm{H}$ & 6.887409 & 0.429070 & -2.994355 \\
\hline 0 & -1.317334 & 1.927432 & -1.141245 \\
\hline $\mathrm{C}$ & 2.443773 & -1.865510 & 2.559221 \\
\hline $\mathrm{C}$ & 3.011853 & 0.509094 & 1.912703 \\
\hline $\mathrm{C}$ & 4.201983 & -1.226123 & 0.904499 \\
\hline 0 & 4.140249 & 0.146894 & 1.238639 \\
\hline
\end{tabular}

\begin{tabular}{|c|c|c|c|}
\hline C & 3.889462 & -2.094945 & 2.110150 \\
\hline $\mathrm{C}$ & 2.167213 & -0.361848 & 2.578885 \\
\hline $\mathrm{H}$ & 3.474182 & -1.423580 & 0.090938 \\
\hline $\mathrm{H}$ & 4.583403 & -1.831304 & 2.923796 \\
\hline $\mathrm{H}$ & 1.572653 & 0.053514 & 3.394869 \\
\hline $\mathrm{H}$ & 1.774382 & -2.362520 & 1.835815 \\
\hline $\mathrm{H}$ & 3.023877 & 1.576272 & 2.135089 \\
\hline $\mathrm{H}$ & 5.212375 & -1.391145 & 0.511955 \\
\hline $\mathrm{H}$ & 4.060795 & -3.151307 & 1.860551 \\
\hline B & 2.085491 & -2.387686 & 3.989879 \\
\hline 0 & 0.971074 & -3.139297 & 4.261724 \\
\hline C & 0.723890 & -2.998609 & 5.675618 \\
\hline C & 2.152129 & -2.706361 & 6.225969 \\
\hline 0 & 2.772454 & -2.023139 & 5.118102 \\
\hline $\mathrm{C}$ & 2.963711 & -3.968477 & 6.479374 \\
\hline $\mathrm{H}$ & 2.928343 & -4.646109 & 5.616456 \\
\hline $\mathrm{H}$ & 2.606982 & -4.511483 & 7.364393 \\
\hline $\mathrm{H}$ & 4.010867 & -3.687684 & 6.645831 \\
\hline C & 0.082532 & -4.266466 & 6.199547 \\
\hline $\mathrm{H}$ & -0.920110 & -4.378090 & 5.767083 \\
\hline $\mathrm{H}$ & -0.024438 & -4.224245 & 7.292735 \\
\hline $\mathrm{H}$ & 0.666607 & -5.155775 & 5.938879 \\
\hline C & -0.234400 & -1.824723 & 5.835958 \\
\hline $\mathrm{H}$ & -1.144788 & -2.025829 & 5.256660 \\
\hline $\mathrm{H}$ & 0.205200 & -0.892535 & 5.453250 \\
\hline $\mathrm{H}$ & -0.516903 & -1.670379 & 6.885607 \\
\hline C & 2.193997 & -1.805007 & 7.441932 \\
\hline $\mathrm{H}$ & 1.757049 & -0.823066 & 7.231368 \\
\hline $\mathrm{H}$ & 3.234518 & -1.651415 & 7.753992 \\
\hline $\mathrm{H}$ & 1.650153 & -2.259484 & 8.282222 \\
\hline
\end{tabular}

SCF energy: -2953.040980060

M06-D3 (Gas Phase)/6-31G(d)+LANL2DZ (Pd) opt. No imaginary frequency 


\begin{tabular}{|c|c|c|c|}
\hline \multicolumn{4}{|c|}{ Product_3 } \\
\hline $\mathrm{C}$ & -0.093818 & -0.252767 & 0.988936 \\
\hline $\mathrm{C}$ & 1.008426 & 1.753273 & 1.980445 \\
\hline $\mathrm{C}$ & -0.823167 & 0.857827 & 3.103780 \\
\hline 0 & 0.039270 & 1.970274 & 2.902712 \\
\hline $\mathrm{C}$ & -1.289907 & 0.266214 & 1.788170 \\
\hline $\mathrm{C}$ & 1.045634 & 0.731196 & 1.119972 \\
\hline $\mathrm{H}$ & -0.278545 & 0.096620 & 3.689475 \\
\hline $\mathrm{H}$ & -1.809276 & 1.048629 & 1.215521 \\
\hline $\mathrm{H}$ & 1.899358 & 0.647413 & 0.448551 \\
\hline $\mathrm{H}$ & 0.227258 & -1.237807 & 1.374491 \\
\hline $\mathrm{H}$ & 1.775676 & 2.524626 & 2.027187 \\
\hline $\mathrm{H}$ & -1.653848 & 1.233395 & 3.711824 \\
\hline $\mathrm{H}$ & -2.022607 & -0.530705 & 1.974998 \\
\hline B & -0.380952 & -0.387315 & -0.547349 \\
\hline 0 & 0.509031 & -0.968636 & -1.414364 \\
\hline C & 0.122034 & -0.544693 & -2.736966 \\
\hline $\mathrm{C}$ & -1.400029 & -0.264176 & -2.560528 \\
\hline 0 & -1.477542 & 0.137942 & -1.176821 \\
\hline $\mathrm{C}$ & -2.258224 & -1.512378 & -2.704536 \\
\hline $\mathrm{H}$ & -1.863233 & -2.340588 & -2.102026 \\
\hline $\mathrm{H}$ & -2.324922 & -1.841873 & -3.749558 \\
\hline $\mathrm{H}$ & -3.271110 & -1.287789 & -2.348684 \\
\hline $\mathrm{C}$ & 0.461624 & -1.636428 & -3.729314 \\
\hline $\mathrm{H}$ & 1.549892 & -1.766032 & -3.778423 \\
\hline $\mathrm{H}$ & 0.105862 & -1.371781 & -4.735113 \\
\hline $\mathrm{H}$ & 0.021073 & -2.597456 & -3.443389 \\
\hline $\mathrm{C}$ & 0.927637 & 0.711447 & -3.040125 \\
\hline $\mathrm{H}$ & 1.994549 & 0.478595 & -2.934868 \\
\hline $\mathrm{H}$ & 0.688916 & 1.518472 & -2.333935 \\
\hline $\mathrm{H}$ & 0.752507 & 1.075369 & -4.060870 \\
\hline $\mathrm{C}$ & -1.949791 & 0.849404 & -3.427092 \\
\hline $\mathrm{H}$ & -1.456145 & 1.805027 & -3.221394 \\
\hline $\mathrm{H}$ & -3.022071 & 0.975407 & -3.232282 \\
\hline $\mathrm{H}$ & -1.824363 & 0.610395 & -4.492630 \\
\hline \multicolumn{4}{|c|}{$\begin{array}{l}\mathrm{SCF} \text { energy: }-680.770002913 \\
\mathrm{SC}\end{array}$} \\
\hline \multicolumn{4}{|c|}{$\begin{array}{l}\text { M06-D3 (Gas Phase) /6-31G (d) +LANL2DZ (Pd) opt. } \\
\text { No imaginary frequency }\end{array}$} \\
\hline
\end{tabular}

\begin{tabular}{|c|c|c|c|}
\hline \multicolumn{4}{|c|}{ Product_5 } \\
\hline $\mathrm{H}$ & 0.281446 & 4.295474 & 3.641612 \\
\hline $\mathrm{H}$ & 2.033696 & 2.693751 & 2.620467 \\
\hline $\mathrm{H}$ & 1.850110 & 2.555561 & 4.362184 \\
\hline $\mathrm{H}$ & 4.418097 & 0.219529 & 0.295408 \\
\hline $\mathrm{C}$ & 1.324822 & 2.394730 & 3.407153 \\
\hline $\mathrm{C}$ & 0.077821 & 3.256994 & 3.354250 \\
\hline $\mathrm{H}$ & 5.961754 & -0.191391 & 2.138873 \\
\hline $\mathrm{H}$ & -0.330301 & 3.263561 & 2.324487 \\
\hline 0 & 3.335319 & 0.213162 & 2.630673 \\
\hline $\mathrm{C}$ & 3.921892 & -0.730808 & 0.527098 \\
\hline $\mathrm{H}$ & 2.891719 & -0.674553 & 0.152139 \\
\hline 0 & -0.898435 & 2.779760 & 4.255412 \\
\hline $\mathrm{H}$ & 4.445343 & -1.533138 & -0.009006 \\
\hline $\mathrm{C}$ & 5.384565 & -1.045105 & 2.515285 \\
\hline $\mathrm{C}$ & 0.956320 & 0.940132 & 3.254700 \\
\hline $\mathrm{C}$ & 3.952055 & -0.944708 & 2.033987 \\
\hline $\mathrm{H}$ & 5.447789 & -1.037707 & 3.608531 \\
\hline B & 2.015884 & -0.103312 & 2.837380 \\
\hline $\mathrm{H}$ & 5.856575 & -1.964836 & 2.141797 \\
\hline $\mathrm{C}$ & -1.385406 & 1.516936 & 3.882530 \\
\hline $\mathrm{C}$ & -0.314399 & 0.563918 & 3.463165 \\
\hline 0 & 1.745128 & -1.433330 & 2.628571 \\
\hline $\mathrm{H}$ & -2.132491 & 1.612890 & 3.065808 \\
\hline $\mathrm{C}$ & 3.016579 & -2.098991 & 2.505605 \\
\hline $\mathrm{H}$ & -1.930270 & 1.124654 & 4.753781 \\
\hline $\mathrm{H}$ & 3.498184 & -1.809553 & 4.609008 \\
\hline $\mathrm{H}$ & 2.467014 & -2.921717 & 0.570026 \\
\hline $\mathrm{H}$ & -0.608937 & -0.477786 & 3.319543 \\
\hline $\mathrm{C}$ & 2.879258 & -3.249525 & 1.530166 \\
\hline $\mathrm{C}$ & 3.373623 & -2.627740 & 3.887855 \\
\hline $\mathrm{H}$ & 3.853504 & -3.725805 & 1.350700 \\
\hline $\mathrm{H}$ & 4.296326 & -3.222316 & 3.871386 \\
\hline $\mathrm{H}$ & 2.556733 & -3.268020 & 4.242395 \\
\hline $\mathrm{H}$ & 2.203529 & -4.007533 & 1.945469 \\
\hline \multicolumn{4}{|c|}{ SCF energy: -680.770793481} \\
\hline \multicolumn{4}{|c|}{ M06-D3 (Gas Phase) /6-31G (d) +LANL2DZ (Pd) opt. } \\
\hline
\end{tabular}




\section{Cyclohexenyl_Triflate}

$\begin{array}{rrrr}\text { C } & 0.961599 & 0.944473 & 1.417781 \\ \mathrm{C} & 0.595651 & 3.156834 & 2.450847 \\ \mathrm{C} & 0.485113 & 1.069397 & 3.840624 \\ \mathrm{C} & -0.198961 & 2.389050 & 3.503305 \\ \mathrm{C} & 0.536565 & 0.162255 & 2.613715 \\ \mathrm{C} & 1.029701 & 2.269198 & 1.325455 \\ \mathrm{H} & 1.485453 & 3.622438 & 2.908042 \\ \mathrm{H} & 1.511323 & 1.271876 & 4.186725 \\ \mathrm{H} & -0.445387 & -0.299180 & 2.422949 \\ \mathrm{H} & 1.416125 & 2.716708 & 0.409975 \\ \mathrm{H} & 0.000699 & 3.990695 & 2.050461 \\ \mathrm{H} & -0.031319 & 0.557351 & 4.662516 \\ \mathrm{H} & 1.242392 & -0.668292 & 2.762053 \\ \mathrm{O} & 1.442393 & 0.173063 & 0.329518 \\ \mathrm{~S} & 0.392228 & -0.497031 & -0.688318 \\ \mathrm{O} & 1.107383 & -1.537296 & -1.379806 \\ \mathrm{O} & -0.891315 & -0.694520 & -0.055191 \\ \mathrm{C} & 0.184123 & 0.883880 & -1.899020 \\ \mathrm{~F} & 1.368329 & 1.337871 & -2.263705 \\ \mathrm{~F} & -0.456438 & 0.405354 & -2.948027 \\ \mathrm{~F} & -0.518933 & 1.863507 & -1.358482 \\ \mathrm{H} & -0.325414 & 3.004860 & 4.403387 \\ \mathrm{H} & -1.208911 & 2.181806 & 3.115096 \\ - & -1030\end{array}$

SCF energy: -1195.038218220

M06-D3 (Gas Phase) /6-31G(d) +LANL2DZ (Pd) opt. No imaginary frequency

\begin{tabular}{|c|c|c|c|}
\hline \multicolumn{4}{|c|}{ Int-S1 } \\
\hline $\mathrm{Pd}$ & -0.645952 & -0.286095 & 0.620392 \\
\hline $\mathrm{P}$ & 1.673537 & -0.135892 & 1.205991 \\
\hline $\mathrm{P}$ & -1.004108 & 1.289102 & -1.162578 \\
\hline $\mathrm{C}$ & 2.174145 & 0.902011 & 2.626649 \\
\hline $\mathrm{C}$ & 2.850864 & 2.405360 & 4.878810 \\
\hline $\mathrm{C}$ & 3.438489 & 1.487459 & 2.727788 \\
\hline $\mathrm{C}$ & 1.251965 & 1.079166 & 3.659632 \\
\hline $\mathrm{C}$ & 1.590087 & 1.823805 & 4.785111 \\
\hline $\mathrm{C}$ & 3.773941 & 2.238127 & 3.849077 \\
\hline $\mathrm{H}$ & 4.162140 & 1.350434 & 1.922723 \\
\hline $\mathrm{H}$ & 0.260638 & 0.630035 & 3.568246 \\
\hline $\mathrm{H}$ & 0.865110 & 1.956381 & 5.586718 \\
\hline $\mathrm{H}$ & 4.760501 & 2.693778 & 3.921430 \\
\hline $\mathrm{H}$ & 3.115336 & 2.993938 & 5.756075 \\
\hline $\mathrm{C}$ & 2.702361 & 0.482457 & -0.185957 \\
\hline $\mathrm{C}$ & 3.848337 & 1.372536 & -2.585218 \\
\hline $\mathrm{C}$ & 2.374561 & 1.733438 & -0.728988 \\
\hline $\mathrm{C}$ & 3.650697 & -0.290715 & -0.857420 \\
\hline $\mathrm{C}$ & 4.222347 & 0.146184 & -2.049231 \\
\hline $\mathrm{C}$ & 2.930521 & 2.180346 & -1.920713 \\
\hline $\mathrm{H}$ & 3.926285 & -1.263334 & -0.451645 \\
\hline $\mathrm{H}$ & 4.950730 & -0.478937 & -2.562040 \\
\hline $\mathrm{H}$ & 2.637067 & 3.145599 & -2.330135 \\
\hline $\mathrm{H}$ & 4.274543 & 1.713602 & -3.527218 \\
\hline $\mathrm{C}$ & 2.433073 & -1.761731 & 1.591334 \\
\hline $\mathrm{C}$ & 3.318235 & -4.354849 & 2.164495 \\
\hline $\mathrm{C}$ & 2.155737 & -2.822222 & 0.719314 \\
\hline $\mathrm{C}$ & 3.163026 & -2.018158 & 2.754144 \\
\hline $\mathrm{C}$ & 3.601343 & -3.309894 & 3.037041 \\
\hline $\mathrm{C}$ & 2.596398 & -4.108175 & 0.998552 \\
\hline $\mathrm{H}$ & 1.566160 & -2.637253 & -0.179483 \\
\hline $\mathrm{H}$ & 3.384432 & -1.209006 & 3.449664 \\
\hline $\mathrm{H}$ & 4.164049 & -3.498528 & 3.950306 \\
\hline $\mathrm{H}$ & 2.347228 & -4.922275 & 0.319534 \\
\hline $\mathrm{H}$ & 3.650879 & -5.365440 & 2.395833 \\
\hline $\mathrm{C}$ & -0.230554 & 0.598663 & -2.681516 \\
\hline $\mathrm{C}$ & 0.956399 & -0.562960 & -4.929136 \\
\hline $\mathrm{C}$ & 0.019104 & 1.364374 & -3.823728 \\
\hline $\mathrm{C}$ & 0.111410 & -0.757115 & -2.682462 \\
\hline $\mathrm{C}$ & 0.698158 & -1.337178 & -3.802478 \\
\hline
\end{tabular}

\begin{tabular}{|c|c|c|c|}
\hline C & 0.614540 & 0.787187 & -4.939424 \\
\hline $\mathrm{H}$ & -0.241171 & 2.423690 & -3.835076 \\
\hline $\mathrm{H}$ & -0.081688 & -1.351938 & -1.785172 \\
\hline $\mathrm{H}$ & 0.960699 & -2.393708 & -3.788761 \\
\hline $\mathrm{H}$ & 0.813236 & 1.393955 & -5.821763 \\
\hline $\mathrm{H}$ & 1.426027 & -1.012129 & -5.803015 \\
\hline C & -0.487866 & 3.058169 & -1.234290 \\
\hline C & 0.350367 & 5.744154 & -1.172778 \\
\hline C & 0.716670 & 3.434082 & -0.625037 \\
\hline C & -1.252868 & 4.068246 & -1.828199 \\
\hline C & -0.840291 & 5.396393 & -1.803555 \\
\hline C & 1.132461 & 4.759104 & -0.581111 \\
\hline $\mathrm{H}$ & -2.194951 & 3.806144 & -2.309805 \\
\hline $\mathrm{H}$ & -1.454776 & 6.161587 & -2.274434 \\
\hline $\mathrm{H}$ & 2.068696 & 4.992903 & -0.076956 \\
\hline $\mathrm{H}$ & 0.673304 & 6.783172 & -1.141473 \\
\hline C & -2.784090 & 1.375014 & -1.595360 \\
\hline C & -5.560249 & 1.259236 & -1.953279 \\
\hline C & -3.643918 & 2.089629 & -0.750467 \\
\hline C & -3.335086 & 0.594708 & -2.614759 \\
\hline C & -4.715704 & 0.534382 & -2.787216 \\
\hline C & -5.019469 & 2.043171 & -0.936027 \\
\hline $\mathrm{H}$ & -3.227111 & 2.683883 & 0.065679 \\
\hline $\mathrm{H}$ & -2.682423 & 0.021756 & -3.274118 \\
\hline $\mathrm{H}$ & -5.131474 & -0.081670 & -3.583192 \\
\hline $\mathrm{H}$ & -5.673746 & 2.609766 & -0.275057 \\
\hline $\mathrm{H}$ & -6.639046 & 1.211644 & -2.091975 \\
\hline O & 1.490303 & 2.486796 & 0.003903 \\
\hline C & -1.546393 & -2.132677 & 1.335830 \\
\hline C & -3.654486 & -0.734763 & 1.380428 \\
\hline C & -2.458569 & -1.361949 & 3.504623 \\
\hline C & -3.806423 & -1.283420 & 2.796624 \\
\hline $\mathrm{C}$ & -1.564924 & -2.384955 & 2.812147 \\
\hline C & -2.524007 & -1.419683 & 0.636800 \\
\hline $\mathrm{H}$ & -3.496252 & 0.355484 & 1.419570 \\
\hline $\mathrm{H}$ & -1.974504 & -0.370014 & 3.482133 \\
\hline $\mathrm{H}$ & -1.969558 & -3.398332 & 2.995563 \\
\hline $\mathrm{H}$ & -2.702092 & -1.678754 & -0.407915 \\
\hline $\mathrm{H}$ & -4.587590 & -0.873717 & 0.813945 \\
\hline $\mathrm{H}$ & -2.581989 & -1.634414 & 4.561411 \\
\hline $\mathrm{H}$ & -0.539969 & -2.378018 & 3.213804 \\
\hline 0 & -0.810085 & -3.147539 & 0.616326 \\
\hline S & -1.494896 & -4.532416 & 0.207957 \\
\hline 0 & -0.613508 & -5.616965 & 0.575767 \\
\hline O & -2.911885 & -4.546761 & 0.496997 \\
\hline C & -1.293553 & -4.341471 & -1.613842 \\
\hline $\mathrm{F}$ & -0.013078 & -4.137469 & -1.895378 \\
\hline $\mathrm{F}$ & -1.703520 & -5.448837 & -2.203643 \\
\hline $\mathrm{F}$ & -2.003649 & -3.317265 & -2.058232 \\
\hline $\mathrm{H}$ & -4.512725 & -0.664239 & 3.366927 \\
\hline $\mathrm{H}$ & -4.235941 & -2.298187 & 2.745964 \\
\hline
\end{tabular}

SCF energy: -3467.317803950

M06-D3 (Gas Phase) /6-31G(d) +LANL2DZ (Pd) opt. No imaginary frequency 


\begin{tabular}{|c|c|c|c|}
\hline \multicolumn{4}{|l|}{ TS-S1 } \\
\hline $\mathrm{Pd}$ & 0.531007 & -0.544280 & 0.684344 \\
\hline $\mathrm{P}$ & 1. 341385 & 0.277611 & -1.410108 \\
\hline $\mathrm{P}$ & -1.763555 & 0.244300 & 0.837634 \\
\hline $\mathrm{C}$ & 1.631608 & -0.830652 & -2.827428 \\
\hline $\mathrm{C}$ & 2.121058 & -2.605356 & -4.917760 \\
\hline $\mathrm{C}$ & 1.433945 & -0.424595 & -4.150374 \\
\hline $\mathrm{C}$ & 2.083701 & -2.123733 & -2.558737 \\
\hline $\mathrm{C}$ & 2.323129 & -3.010770 & -3.602778 \\
\hline $\mathrm{C}$ & 1.681009 & -1.311690 & -5.191437 \\
\hline $\mathrm{H}$ & 1.082673 & 0.585636 & -4.364117 \\
\hline $\mathrm{H}$ & 2.240183 & -2.446636 & -1.528411 \\
\hline $\mathrm{H}$ & 2.640689 & -4.025884 & -3.373810 \\
\hline $\mathrm{H}$ & 1.522907 & -0.994515 & -6.221359 \\
\hline $\mathrm{H}$ & 2.298822 & -3.302144 & -5.735669 \\
\hline $\mathrm{C}$ & -2.996059 & -0.332764 & -0.399581 \\
\hline $\mathrm{C}$ & -4.780998 & -1.351903 & -2.316800 \\
\hline $\mathrm{C}$ & -2.642185 & -0.420158 & -1.751852 \\
\hline $\mathrm{C}$ & -4.276949 & -0.761724 & -0.036361 \\
\hline $\mathrm{C}$ & -5.165556 & -1.260037 & -0.983003 \\
\hline $\mathrm{C}$ & -3.514777 & -0.929398 & -2.703770 \\
\hline $\mathrm{H}$ & -4.574521 & -0.717447 & 1.011423 \\
\hline $\mathrm{H}$ & -6.155392 & -1.589922 & -0.673523 \\
\hline $\mathrm{H}$ & -3.175264 & -0.995935 & -3.735606 \\
\hline $\mathrm{H}$ & -5.465880 & -1.756507 & -3.059646 \\
\hline $\mathrm{C}$ & -2.467373 & -0.402664 & 2.403556 \\
\hline $\mathrm{C}$ & -3.364266 & -1.510361 & 4.805949 \\
\hline $\mathrm{C}$ & -3.029828 & 0.412732 & 3.385964 \\
\hline $\mathrm{C}$ & -2.359971 & -1.782579 & 2.629798 \\
\hline $\mathrm{C}$ & -2.809567 & -2.330215 & 3.823693 \\
\hline $\mathrm{C}$ & -3.473896 & -0.142510 & 4.585088 \\
\hline $\mathrm{H}$ & -3.119732 & 1.486379 & 3.220619 \\
\hline $\mathrm{H}$ & -1.912221 & -2.425274 & 1.867441 \\
\hline $\mathrm{H}$ & -2.715359 & -3.402474 & 3.989008 \\
\hline $\mathrm{H}$ & -3.907920 & 0.501761 & 5.348441 \\
\hline $\mathrm{H}$ & -3.709230 & -1.940595 & 5.744959 \\
\hline $\mathrm{C}$ & -2.048078 & 2.050147 & 0.938939 \\
\hline $\mathrm{C}$ & -2.335903 & 4.819069 & 1.150878 \\
\hline $\mathrm{C}$ & -0.983849 & 2.840178 & 1. 381295 \\
\hline $\mathrm{C}$ & -3.262143 & 2.659026 & 0.610681 \\
\hline $\mathrm{C}$ & -3.402852 & 4.038523 & 0.712799 \\
\hline $\mathrm{C}$ & -1.127544 & 4.218985 & 1.492051 \\
\hline $\mathrm{H}$ & -0.036885 & 2.355419 & 1.632354 \\
\hline $\mathrm{H}$ & -4.097232 & 2.049438 & 0.262175 \\
\hline $\mathrm{H}$ & -4.349576 & 4.507246 & 0.448237 \\
\hline $\mathrm{H}$ & -0.291333 & 4.826936 & 1.833748 \\
\hline $\mathrm{H}$ & -2.447385 & 5.899694 & 1.225774 \\
\hline $\mathrm{C}$ & 0.270691 & 1.605776 & -2.073687 \\
\hline $\mathrm{C}$ & -1.602070 & 3.562249 & -2.779507 \\
\hline $\mathrm{C}$ & -1.073959 & 1.276489 & -2.291345 \\
\hline $\mathrm{C}$ & 0.660335 & 2.934265 & -2.245561 \\
\hline $\mathrm{C}$ & -0.268618 & 3.910685 & -2.595124 \\
\hline $\mathrm{C}$ & -2.012272 & 2.239885 & -2.639252 \\
\hline $\mathrm{H}$ & 1.702360 & 3.209912 & -2.087047 \\
\hline $\mathrm{H}$ & 0.049855 & 4.944392 & -2.714100 \\
\hline $\mathrm{H}$ & -3.052875 & 1.954625 & -2.786523 \\
\hline $\mathrm{H}$ & -2.336210 & 4.323453 & -3.038354 \\
\hline $\mathrm{C}$ & 2.955105 & 1.120678 & -1.180612 \\
\hline $\mathrm{C}$ & 5.426929 & 2.299520 & -0.600577 \\
\hline $\mathrm{C}$ & 3.095537 & 1.982657 & -0.085272 \\
\hline $\mathrm{C}$ & 4.067347 & 0.861374 & -1.983911 \\
\hline $\mathrm{C}$ & 5.298385 & 1.443521 & -1.688569 \\
\hline $\mathrm{C}$ & 4.318012 & 2.577843 & 0.196756 \\
\hline $\mathrm{H}$ & 2.233042 & 2.170793 & 0.558983 \\
\hline $\mathrm{H}$ & 3.975823 & 0.189129 & -2.836522 \\
\hline $\mathrm{H}$ & 6.161434 & 1.223115 & -2.314827 \\
\hline $\mathrm{H}$ & 4.411744 & 3.246421 & 1.051632 \\
\hline $\mathrm{H}$ & 6.391152 & 2.749809 & -0.370387 \\
\hline $\mathrm{C}$ & 2.123635 & -1.377288 & 1.550147 \\
\hline $\mathrm{C}$ & 1.344336 & -1.440146 & 2.662840 \\
\hline $\mathrm{H}$ & 0.447807 & -2.057759 & 2.695306 \\
\hline $\mathrm{C}$ & 3.585652 & -1.217184 & 1.465069 \\
\hline $\mathrm{H}$ & 4.060567 & -2.204132 & 1.562976 \\
\hline
\end{tabular}

$\begin{array}{rrrr}\mathrm{H} & 3.910845 & -0.774217 & 0.513551 \\ \mathrm{C} & 3.980768 & -0.319024 & 2.655738 \\ \mathrm{H} & 3.620554 & 0.705258 & 2.464772 \\ \mathrm{H} & 5.076935 & -0.265170 & 2.704586 \\ \mathrm{C} & 3.393478 & -0.838714 & 3.963015 \\ \mathrm{C} & 1.865943 & -0.819828 & 3.947767 \\ \mathrm{H} & 1.463729 & -1.366336 & 4.811442 \\ \mathrm{H} & 1.497554 & 0.215295 & 4.039028 \\ \mathrm{O} & 1.928152 & -3.338331 & 0.611028 \\ \mathrm{~S} & 0.636675 & -3.923916 & 0.125443 \\ \mathrm{O} & -0.484600 & -2.964885 & 0.140272 \\ \mathrm{O} & 0.779545 & -4.773448 & -1.048385 \\ \mathrm{C} & 0.187283 & -5.072244 & 1.486915 \\ \mathrm{~F} & 1.151123 & -5.953088 & 1.704994 \\ \mathrm{~F} & -0.021019 & -4.386039 & 2.617277 \\ \mathrm{~F} & -0.931604 & -5.716954 & 1.187227 \\ \mathrm{O} & -1.382510 & -0.051848 & -2.164949 \\ \mathrm{H} & 3.738553 & -1.872889 & 4.122538 \\ \mathrm{H} & 3.767414 & -0.246261 & 4.809898 \\ ----------------------------------------\end{array}$

SCF energy: -3467.284740190

M06-D3 (Gas Phase) /6-31G(d) +LANL2DZ (Pd) opt. 1 imaginary frequency: $132 i \mathrm{~cm}-1$ 


\begin{tabular}{|c|c|c|c|}
\hline \multicolumn{4}{|c|}{ Int-S2 } \\
\hline $\mathrm{Pd}$ & 1.798674 & 0.861465 & 1.329557 \\
\hline $\mathrm{P}$ & 2.306770 & 1.006133 & -1.111514 \\
\hline $\mathrm{P}$ & -0.484206 & 1.176471 & 1.499930 \\
\hline $\mathrm{C}$ & 1.430829 & -0.189369 & -2.181359 \\
\hline $\mathrm{C}$ & 0.190839 & -2.148389 & -3.730656 \\
\hline $\mathrm{C}$ & 1.070333 & 0.083880 & -3.502861 \\
\hline $\mathrm{C}$ & 1.173596 & -1.454099 & -1.640670 \\
\hline $\mathrm{C}$ & 0.558128 & -2.429350 & -2.417693 \\
\hline $\mathrm{C}$ & 0.449185 & -0.892931 & -4.273640 \\
\hline $\mathrm{H}$ & 1.279050 & 1.065108 & -3.931973 \\
\hline $\mathrm{H}$ & 1.478299 & -1.685269 & -0.616867 \\
\hline $\mathrm{H}$ & 0.368918 & -3.413242 & -1.992432 \\
\hline $\mathrm{H}$ & 0.168219 & -0.673093 & -5.302782 \\
\hline $\mathrm{H}$ & -0.294417 & -2.912888 & -4.335725 \\
\hline $\mathrm{C}$ & -1.293638 & 1.181349 & -0.145250 \\
\hline $\mathrm{C}$ & -2.311561 & 1.133474 & -2.754144 \\
\hline $\mathrm{C}$ & -0.873158 & 2.108031 & -1.104160 \\
\hline $\mathrm{C}$ & -2.229689 & 0.217697 & -0.529755 \\
\hline $\mathrm{C}$ & -2.737083 & 0.191735 & -1.824245 \\
\hline $\mathrm{C}$ & -1.380088 & 2.101381 & -2.397817 \\
\hline $\mathrm{H}$ & -2.554719 & -0.532735 & 0.188631 \\
\hline $\mathrm{H}$ & -3.455973 & -0.574128 & -2.107133 \\
\hline $\mathrm{H}$ & -1.030278 & 2.838181 & -3.119037 \\
\hline $\mathrm{H}$ & -2.699110 & 1.113576 & -3.771294 \\
\hline $\mathrm{C}$ & -1.248113 & -0.281846 & 2.289647 \\
\hline $\mathrm{C}$ & -2.410642 & -2.590998 & 3.334278 \\
\hline $\mathrm{C}$ & -2.512445 & -0.223530 & 2.885616 \\
\hline $\mathrm{C}$ & -0.574722 & -1.503600 & 2.212095 \\
\hline $\mathrm{C}$ & -1.157112 & -2.653982 & 2.734443 \\
\hline $\mathrm{C}$ & -3.088247 & -1.375822 & 3.408812 \\
\hline $\mathrm{H}$ & -3.048147 & 0.724542 & 2.939444 \\
\hline $\mathrm{H}$ & 0.423831 & -1.559070 & 1.774432 \\
\hline $\mathrm{H}$ & -0.614378 & -3.595927 & 2.679880 \\
\hline $\mathrm{H}$ & -4.069917 & -1.324343 & 3.877068 \\
\hline $\mathrm{H}$ & -2.862470 & -3.490435 & 3.749945 \\
\hline $\mathrm{C}$ & -1.207761 & 2.597561 & 2.403100 \\
\hline $\mathrm{C}$ & -2.246045 & 4.759011 & 3.849253 \\
\hline $\mathrm{C}$ & -1.129573 & 2.623544 & 3.802356 \\
\hline $\mathrm{C}$ & -1.830392 & 3.658497 & 1.742924 \\
\hline $\mathrm{C}$ & -2.343508 & 4.733560 & 2.463128 \\
\hline $\mathrm{C}$ & -1.644429 & 3.696113 & 4.518269 \\
\hline $\mathrm{H}$ & -0.670896 & 1.794313 & 4.336959 \\
\hline $\mathrm{H}$ & -1.922485 & 3.651657 & 0.659838 \\
\hline $\mathrm{H}$ & -2.825627 & 5.553100 & 1.932777 \\
\hline $\mathrm{H}$ & -1.573960 & 3.697675 & 5.605157 \\
\hline $\mathrm{H}$ & -2.645898 & 5.602153 & 4.410210 \\
\hline $\mathrm{C}$ & 2.064521 & 2.647322 & -1.929888 \\
\hline C & 1.780934 & 5.230486 & -3.022193 \\
\hline $\mathrm{C}$ & 0.985740 & 3.473253 & -1.592648 \\
\hline $\mathrm{C}$ & 2.990859 & 3.153141 & -2.850304 \\
\hline $\mathrm{C}$ & 2.850076 & 4. 424047 & -3.397548 \\
\hline $\mathrm{C}$ & 0.842254 & 4.751495 & -2.116073 \\
\hline $\mathrm{H}$ & 3.846684 & 2.540063 & -3.130719 \\
\hline $\mathrm{H}$ & 3.587302 & 4.787229 & -4.110892 \\
\hline $\mathrm{H}$ & -0.010153 & 5.350186 & -1.799874 \\
\hline $\mathrm{H}$ & 1.673299 & 6.231566 & -3.435586 \\
\hline $\mathrm{C}$ & 4.072697 & 0.714443 & -1.474478 \\
\hline $\mathrm{C}$ & 6.797892 & 0.354511 & -1.921184 \\
\hline $\mathrm{C}$ & 5.009105 & 1.365185 & -0.666513 \\
\hline $\mathrm{C}$ & 4.507659 & -0.115977 & -2.506638 \\
\hline $\mathrm{C}$ & 5.869432 & -0.294476 & -2.727205 \\
\hline $\mathrm{C}$ & 6.366956 & 1.188043 & -0.892184 \\
\hline $\mathrm{H}$ & 4.671066 & 1.995300 & 0.156853 \\
\hline $\mathrm{H}$ & 3.783787 & -0.642164 & -3.128292 \\
\hline $\mathrm{H}$ & 6.204369 & -0.954237 & -3.525827 \\
\hline $\mathrm{H}$ & 7.091445 & 1.688261 & -0.251989 \\
\hline $\mathrm{H}$ & 7.863278 & 0.204199 & -2.088883 \\
\hline $\mathrm{C}$ & 1.738189 & 1.034771 & 3.353878 \\
\hline $\mathrm{C}$ & 1.985930 & 2.275384 & 3.775877 \\
\hline $\mathrm{H}$ & 1.960047 & 3.116116 & 3.075240 \\
\hline $\mathrm{C}$ & 1.721642 & -0.170524 & 4.238417 \\
\hline $\mathrm{H}$ & 0.678373 & -0.431712 & 4.496590 \\
\hline
\end{tabular}

$\begin{array}{crrr}\mathrm{H} & 2.126138 & -1.047804 & 3.718590 \\ \mathrm{C} & 2.515501 & 0.119487 & 5.512238 \\ \mathrm{H} & 3.582746 & 0.193938 & 5.249253 \\ \mathrm{H} & 2.414886 & -0.719724 & 6.214832 \\ \mathrm{C} & 2.045974 & 1.424200 & 6.141938 \\ \mathrm{C} & 2.307692 & 2.605067 & 5.211631 \\ \mathrm{H} & 1.717772 & 3.482510 & 5.524544 \\ \mathrm{H} & 3.364294 & 2.915536 & 5.280028 \\ \mathrm{O} & 3.806187 & -0.125882 & 1.688339 \\ \mathrm{~S} & 3.803353 & -1.590738 & 1.352266 \\ \mathrm{O} & 2.508841 & -2.238661 & 1.618826 \\ \mathrm{O} & 4.453589 & -1.935239 & 0.094899 \\ \mathrm{C} & 4.915313 & -2.248462 & 2.656430 \\ \mathrm{~F} & 6.107868 & -1.675742 & 2.575517 \\ \mathrm{~F} & 4.412043 & -2.014454 & 3.866241 \\ \mathrm{~F} & 5.054489 & -3.558390 & 2.504622 \\ \mathrm{O} & 0.041944 & 3.043066 & -0.684259 \\ \mathrm{H} & 0.962923 & 1.350332 & 6.344390 \\ \mathrm{H} & 2.530881 & 1.593589 & 7.113600 \\ ---------------------------------------\end{array}$

SCF energy: -3467.320064320

M06-D3 (Gas Phase)/6-31G(d)+LANL2DZ (Pd) opt. No imaginary frequency 


\begin{tabular}{|c|c|c|c|}
\hline \multicolumn{4}{|c|}{ Int-s3 } \\
\hline $\mathrm{Pd}$ & 1.583467 & 1.281665 & 1.334722 \\
\hline $\mathrm{P}$ & 1.902156 & 1.102665 & -1.224774 \\
\hline $\mathrm{P}$ & -0.760157 & 1.220111 & 1.562463 \\
\hline $\mathrm{C}$ & 1.394082 & -0.444441 & -2.058865 \\
\hline $\mathrm{C}$ & 0.622685 & -2.851399 & -3.240461 \\
\hline $\mathrm{C}$ & 1.244014 & -0.531175 & -3.447832 \\
\hline $\mathrm{C}$ & 1.160285 & -1.572313 & -1.271178 \\
\hline $\mathrm{C}$ & 0.775286 & -2.772750 & -1.860979 \\
\hline $\mathrm{C}$ & 0.861511 & -1.730449 & -4.034323 \\
\hline $\mathrm{H}$ & 1.418850 & 0.345831 & -4.072238 \\
\hline $\mathrm{H}$ & 1.289288 & -1.512972 & -0.188420 \\
\hline $\mathrm{H}$ & 0.591618 & -3.645203 & -1.235609 \\
\hline $\mathrm{H}$ & 0.746459 & -1.791945 & -5.114986 \\
\hline $\mathrm{H}$ & 0.317385 & -3.788561 & -3.702541 \\
\hline $\mathrm{C}$ & -1.551451 & 0.448956 & 0.106421 \\
\hline $\mathrm{C}$ & -2.535676 & -0.588581 & -2.292009 \\
\hline $\mathrm{C}$ & -1.429149 & 1.157338 & -1.092619 \\
\hline $\mathrm{C}$ & -2.221372 & -0.776200 & 0.089732 \\
\hline $\mathrm{C}$ & -2.709354 & -1.294333 & -1.105344 \\
\hline $\mathrm{C}$ & -1.906004 & 0.651699 & -2.293755 \\
\hline $\mathrm{H}$ & -2.359413 & -1.332936 & 1.014585 \\
\hline $\mathrm{H}$ & -3.221873 & -2.253660 & -1.109095 \\
\hline $\mathrm{H}$ & -1.779042 & 1.217943 & -3.214833 \\
\hline $\mathrm{H}$ & -2.904327 & -0.999521 & -3.229872 \\
\hline $\mathrm{C}$ & -1.226210 & 0.108925 & 2.934325 \\
\hline $\mathrm{C}$ & -1.695048 & -1.599269 & 5.099793 \\
\hline $\mathrm{C}$ & -1.949992 & 0.555304 & 4.042747 \\
\hline $\mathrm{C}$ & -0.737928 & -1.203683 & 2.923809 \\
\hline $\mathrm{C}$ & -0.973603 & -2.053572 & 3.997766 \\
\hline $\mathrm{C}$ & -2.183485 & -0.297311 & 5.118050 \\
\hline $\mathrm{H}$ & -2.315285 & 1.580713 & 4.079025 \\
\hline $\mathrm{H}$ & -0.158656 & -1.561380 & 2.070333 \\
\hline $\mathrm{H}$ & -0.592487 & -3.073518 & 3.976798 \\
\hline $\mathrm{H}$ & -2.745069 & 0.063500 & 5.977514 \\
\hline $\mathrm{H}$ & -1.874758 & -2.261834 & 5.944098 \\
\hline $\mathrm{C}$ & -1.743927 & 2.735641 & 1.813227 \\
\hline $\mathrm{C}$ & -3.283930 & 5.035678 & 2.145228 \\
\hline $\mathrm{C}$ & -1.129162 & 3.978664 & 1.945466 \\
\hline $\mathrm{C}$ & -3.140580 & 2.651121 & 1.821966 \\
\hline $\mathrm{C}$ & -3.905645 & 3.798319 & 1.994156 \\
\hline $\mathrm{C}$ & -1.896225 & 5.126333 & 2.114826 \\
\hline $\mathrm{H}$ & -0.045234 & 4.048930 & 1.893176 \\
\hline $\mathrm{H}$ & -3.631585 & 1.685277 & 1.694621 \\
\hline $\mathrm{H}$ & -4.991480 & 3.726644 & 2.005780 \\
\hline $\mathrm{H}$ & -1.406655 & 6.093275 & 2.217524 \\
\hline $\mathrm{H}$ & -3.885592 & 5.932745 & 2.278651 \\
\hline $\mathrm{C}$ & 1.161026 & 2.417196 & -2.296931 \\
\hline $\mathrm{C}$ & -0.015130 & 4.444545 & -3.861831 \\
\hline $\mathrm{C}$ & -0.127592 & 2.893143 & -2.037949 \\
\hline $\mathrm{C}$ & 1.836862 & 2.972501 & -3.391685 \\
\hline $\mathrm{C}$ & 1.256575 & 3.969831 & -4.167812 \\
\hline $\mathrm{C}$ & -0.714326 & 3.900507 & -2.791506 \\
\hline $\mathrm{H}$ & 2.839551 & 2.626325 & -3.637619 \\
\hline $\mathrm{H}$ & 1.806303 & 4.381347 & -5.011610 \\
\hline $\mathrm{H}$ & -1.713360 & 4.238136 & -2.522309 \\
\hline $\mathrm{H}$ & -0.466520 & 5.233512 & -4.459665 \\
\hline $\mathrm{C}$ & 3.664971 & 1.313828 & -1.676656 \\
\hline $\mathrm{C}$ & 6.380977 & 1.714064 & -2.199956 \\
\hline $\mathrm{C}$ & 4.274543 & 2.539731 & -1.373568 \\
\hline $\mathrm{C}$ & 4.428041 & 0.293516 & -2.245397 \\
\hline $\mathrm{C}$ & 5.784408 & 0.494109 & -2.499412 \\
\hline $\mathrm{C}$ & 5.621133 & 2.742585 & -1.642691 \\
\hline $\mathrm{H}$ & 3.685224 & 3.340447 & -0.918758 \\
\hline $\mathrm{H}$ & 3.964487 & -0.663264 & -2.487924 \\
\hline $\mathrm{H}$ & 6.373572 & -0.307102 & -2.942197 \\
\hline $\mathrm{H}$ & 6.082967 & 3.700625 & -1.411309 \\
\hline $\mathrm{H}$ & 7.438550 & 1.868333 & -2.404849 \\
\hline $\mathrm{C}$ & 1.532186 & 2.203954 & 3.143596 \\
\hline $\mathrm{C}$ & 1.127750 & 1.754090 & 4. 327648 \\
\hline $\mathrm{H}$ & 0.643975 & 0.780051 & 4.422535 \\
\hline $\mathrm{C}$ & 2.229363 & 3.525823 & 2.949105 \\
\hline $\mathrm{H}$ & 3.320339 & 3.363273 & 2.877083 \\
\hline
\end{tabular}

\begin{tabular}{|c|c|c|c|}
\hline $\mathrm{H}$ & 1.949995 & 4.001400 & 1.994491 \\
\hline C & 1.932316 & 4.462925 & 4.125411 \\
\hline $\mathrm{H}$ & 0.874664 & 4.771799 & 4.082058 \\
\hline $\mathrm{H}$ & 2.539038 & 5.374071 & 4.036932 \\
\hline C & 2.201916 & 3.747788 & 5.441306 \\
\hline C & 1.292712 & 2.535501 & 5.605957 \\
\hline $\mathrm{H}$ & 1.673482 & 1.871992 & 6.397033 \\
\hline $\mathrm{H}$ & 0.289479 & 2.849558 & 5.945213 \\
\hline 0 & -0.833708 & 2.385509 & -0.972804 \\
\hline $\mathrm{N}$ & 3.752188 & 0.321606 & 2.105999 \\
\hline $\mathrm{C}$ & 3.934931 & 0.317721 & 3.571220 \\
\hline $\mathrm{H}$ & 3.070640 & -0.102900 & 4.086305 \\
\hline $\mathrm{H}$ & 4.064092 & 1.346655 & 3.922078 \\
\hline $\mathrm{H}$ & 4.833076 & -0.266940 & 3.828601 \\
\hline C & 4.907407 & 1.021703 & 1.536026 \\
\hline $\mathrm{H}$ & 4.848888 & 1.048585 & 0.448986 \\
\hline $\mathrm{H}$ & 5.854441 & 0.542484 & 1.837754 \\
\hline $\mathrm{H}$ & 4.906197 & 2.054884 & 1.902542 \\
\hline C & 3.581844 & -1.026554 & 1.618028 \\
\hline C & 3.169069 & -3.662855 & 0.732506 \\
\hline C & 4.376941 & -1.569424 & 0.606973 \\
\hline C & 2.581248 & -1.826522 & 2.186041 \\
\hline C & 2.380756 & -3.128530 & 1.749556 \\
\hline $\mathrm{C}$ & 4.159980 & -2.873660 & 0.165436 \\
\hline $\mathrm{H}$ & 5.172196 & -0.989843 & 0.146893 \\
\hline $\mathrm{H}$ & 1.948439 & -1.425191 & 2.976203 \\
\hline $\mathrm{H}$ & 1.600921 & -3.732620 & 2.213140 \\
\hline $\mathrm{H}$ & 4.789351 & -3.272365 & -0.628575 \\
\hline $\mathrm{H}$ & 3.013616 & -4.684072 & 0.390791 \\
\hline $\mathrm{H}$ & 3.255506 & 3.423101 & 5.459836 \\
\hline $\mathrm{H}$ & 2.076771 & 4.433518 & 6.289461 \\
\hline
\end{tabular}

SCF energy: -2871.853691470

M06-D3 (Gas Phase) /6-31G(d)+LANL2DZ (Pd) opt. No imaginary frequency 


\begin{tabular}{|c|c|c|c|}
\hline \multicolumn{4}{|c|}{ Int-S4 } \\
\hline $\mathrm{Pd}$ & 0.707261 & 0.182592 & 0.594862 \\
\hline $\mathrm{P}$ & 1.081094 & 0.082914 & -1.815852 \\
\hline $\mathrm{P}$ & -1.545579 & 0.294749 & 1.056698 \\
\hline $\mathrm{C}$ & 0.196763 & -1.054790 & -2.931563 \\
\hline $\mathrm{C}$ & -1.137247 & -2.870457 & -4.574322 \\
\hline $\mathrm{C}$ & 0.104088 & -0.821005 & -4.307844 \\
\hline $\mathrm{C}$ & -0.386304 & -2.200730 & -2.386477 \\
\hline $\mathrm{C}$ & -1.050470 & -3.107326 & -3.206200 \\
\hline $\mathrm{C}$ & -0.559886 & -1.727819 & -5.124833 \\
\hline $\mathrm{H}$ & 0.547104 & 0.077462 & -4.739738 \\
\hline $\mathrm{H}$ & -0.326064 & -2.376381 & -1.311114 \\
\hline $\mathrm{H}$ & -1.506006 & -3.996396 & -2.774261 \\
\hline $\mathrm{H}$ & -0.628235 & -1.542948 & -6.195229 \\
\hline $\mathrm{H}$ & -1.658958 & -3.577773 & -5.216279 \\
\hline $\mathrm{C}$ & -2.396583 & 0.250180 & -0.556830 \\
\hline $\mathrm{C}$ & -3.505777 & 0.079434 & -3.117448 \\
\hline $\mathrm{C}$ & -2.012349 & 1.140267 & -1.569842 \\
\hline $\mathrm{C}$ & -3.358483 & -0.721422 & -0.853471 \\
\hline $\mathrm{C}$ & -3.914022 & -0.805138 & -2.124467 \\
\hline $\mathrm{C}$ & -2.558719 & 1.060060 & -2.844635 \\
\hline $\mathrm{H}$ & -3.666801 & -1.428002 & -0.084610 \\
\hline $\mathrm{H}$ & -4.657110 & -1.569061 & -2.340793 \\
\hline $\mathrm{H}$ & -2.235366 & 1.757976 & -3.615178 \\
\hline $\mathrm{H}$ & -3.927838 & 0.010561 & -4.118073 \\
\hline $\mathrm{C}$ & -2.133140 & -1.197804 & 1.908413 \\
\hline $\mathrm{C}$ & -3.083599 & -3.559528 & 3.043104 \\
\hline $\mathrm{C}$ & -3.302083 & -1.185231 & 2.674953 \\
\hline $\mathrm{C}$ & -1.444538 & -2.400047 & 1.713796 \\
\hline $\mathrm{C}$ & -1.919625 & -3.577640 & 2.278400 \\
\hline $\mathrm{C}$ & -3.772241 & -2.366200 & 3.240340 \\
\hline $\mathrm{H}$ & -3.849233 & -0.254865 & 2.824878 \\
\hline $\mathrm{H}$ & -0.525032 & -2.404103 & 1.123229 \\
\hline $\mathrm{H}$ & -1.377976 & -4.509159 & 2.126987 \\
\hline $\mathrm{H}$ & -4.682426 & -2.353109 & 3.836486 \\
\hline $\mathrm{H}$ & -3.454305 & -4.480246 & 3.489239 \\
\hline $\mathrm{C}$ & -2.232580 & 1.709009 & 1.970052 \\
\hline $\mathrm{C}$ & -3.292173 & 3.849289 & 3.413892 \\
\hline $\mathrm{C}$ & -1.964868 & 1.839548 & 3.339219 \\
\hline $\mathrm{C}$ & -3.039745 & 2.655484 & 1.333758 \\
\hline $\mathrm{C}$ & -3.564215 & 3.722323 & 2.056305 \\
\hline $\mathrm{C}$ & -2.495980 & 2.903210 & 4.055917 \\
\hline $\mathrm{H}$ & -1.340186 & 1.101038 & 3.843249 \\
\hline $\mathrm{H}$ & -3.270385 & 2.558215 & 0.275008 \\
\hline $\mathrm{H}$ & -4.193750 & 4.453828 & 1.553817 \\
\hline $\mathrm{H}$ & -2.286567 & 2.993850 & 5.120317 \\
\hline $\mathrm{H}$ & -3.704190 & 4.685022 & 3.975995 \\
\hline $\mathrm{C}$ & 0.872889 & 1.743006 & -2.567029 \\
\hline $\mathrm{C}$ & 0.552927 & 4.335567 & -3.592078 \\
\hline $\mathrm{C}$ & -0.203362 & 2.551587 & -2.177935 \\
\hline $\mathrm{C}$ & 1.786541 & 2.268711 & -3.486391 \\
\hline $\mathrm{C}$ & 1.627214 & 3.551338 & -4.000244 \\
\hline $\mathrm{C}$ & -0.367738 & 3.835492 & -2.676632 \\
\hline $\mathrm{H}$ & 2.642372 & 1.665751 & -3.791413 \\
\hline $\mathrm{H}$ & 2.349240 & 3.941063 & -4.714390 \\
\hline $\mathrm{H}$ & -1.213670 & 4.427665 & -2.333065 \\
\hline $\mathrm{H}$ & 0.429138 & 5.342551 & -3.984860 \\
\hline $\mathrm{C}$ & 2.857273 & -0.262395 & -2.048407 \\
\hline $\mathrm{C}$ & 5.602142 & -0.727184 & -2.220481 \\
\hline $\mathrm{C}$ & 3.742149 & 0.389177 & -1.178996 \\
\hline $\mathrm{C}$ & 3.357211 & -1.152878 & -2.999516 \\
\hline $\mathrm{C}$ & 4.728255 & -1.383071 & -3.081203 \\
\hline $\mathrm{C}$ & 5.109178 & 0.162866 & -1.268196 \\
\hline $\mathrm{H}$ & 3.355785 & 1.097039 & -0.438046 \\
\hline $\mathrm{H}$ & 2.676893 & -1.670057 & -3.675673 \\
\hline $\mathrm{H}$ & 5.114203 & -2.079044 & -3.823529 \\
\hline $\mathrm{H}$ & 5.791273 & 0.677330 & -0.594146 \\
\hline $\mathrm{H}$ & 6.672563 & -0.910733 & -2.288929 \\
\hline $\mathrm{C}$ & 0.898130 & 0.516236 & 2.569753 \\
\hline $\mathrm{C}$ & 0.936303 & -0.484453 & 3. 452332 \\
\hline $\mathrm{H}$ & 0.494204 & -1.456636 & 3.220947 \\
\hline $\mathrm{C}$ & 1.328813 & 1.929429 & 2.838140 \\
\hline $\mathrm{H}$ & 2.235237 & 2.169117 & 2.256161 \\
\hline
\end{tabular}

$\begin{array}{rrrr}\text { H } & 0.553224 & 2.632131 & 2.493104 \\ \mathrm{C} & 1.599142 & 2.135411 & 4.335036 \\ \mathrm{H} & 0.634871 & 2.224513 & 4.862659 \\ \mathrm{H} & 2.129629 & 3.084527 & 4.485239 \\ \mathrm{C} & 2.383374 & 0.964624 & 4.909952 \\ \mathrm{C} & 1.581209 & -0.326275 & 4.805520 \\ \mathrm{H} & 2.213932 & -1.200371 & 5.015390 \\ \mathrm{H} & 0.785617 & -0.347490 & 5.571788 \\ \mathrm{O} & -1.099567 & 2.103947 & -1.224148 \\ \mathrm{H} & 3.327846 & 0.853453 & 4.353173 \\ \mathrm{H} & 2.656876 & 1.155722 & 5.955657 \\ --------------------------------------\end{array}$

SCF energy: -2505.891869100

M06-D3 (Gas Phase) /6-31G(d) +LANL2DZ (Pd) opt. No imaginary frequency 


\begin{tabular}{|c|c|c|c|}
\hline \multicolumn{4}{|c|}{ Int-S5 } \\
\hline $\mathrm{Pd}$ & 0.030276 & -0.093589 & 0.228940 \\
\hline $\mathrm{P}$ & 0.366962 & -0.199992 & -2.225380 \\
\hline $\mathrm{P}$ & -2.274811 & 0.085042 & 0.512970 \\
\hline $\mathrm{C}$ & -0.753282 & -1.165822 & -3.293237 \\
\hline $\mathrm{C}$ & -2.337699 & -2.804208 & -4.899425 \\
\hline $\mathrm{C}$ & -0.928203 & -0.864214 & -4.647239 \\
\hline $\mathrm{C}$ & -1.377872 & -2.291499 & -2.751148 \\
\hline $\mathrm{C}$ & -2.167027 & -3.109932 & -3.552855 \\
\hline $\mathrm{C}$ & -1.720632 & -1.680465 & -5.445261 \\
\hline $\mathrm{H}$ & -0.443010 & 0.013334 & -5.076970 \\
\hline $\mathrm{H}$ & -1.247858 & -2.525842 & -1.692717 \\
\hline $\mathrm{H}$ & -2.651231 & -3.984656 & -3.122688 \\
\hline $\mathrm{H}$ & -1.854497 & -1.441746 & -6.498734 \\
\hline $\mathrm{H}$ & -2.955646 & -3.442899 & -5.527861 \\
\hline $\mathrm{C}$ & -3.097926 & 0.354447 & -1.100602 \\
\hline C & -4.203966 & 0.729105 & -3.646095 \\
\hline $\mathrm{C}$ & -2.588576 & 1.320309 & -1.975467 \\
\hline $\mathrm{C}$ & -4.170057 & -0.428978 & -1.538331 \\
\hline $\mathrm{C}$ & -4.722617 & -0.244489 & -2.799490 \\
\hline $\mathrm{C}$ & -3.135711 & 1.517832 & -3.237286 \\
\hline $\mathrm{H}$ & -4.564167 & -1.212855 & -0.894899 \\
\hline $\mathrm{H}$ & -5.550824 & -0.870553 & -3.123341 \\
\hline $\mathrm{H}$ & -2.715310 & 2.275813 & -3.895762 \\
\hline $\mathrm{H}$ & -4.627778 & 0.875776 & -4.637536 \\
\hline $\mathrm{C}$ & -2.896355 & -1.526284 & 1.094644 \\
\hline $\mathrm{C}$ & -3.810950 & -4.047276 & 1.878064 \\
\hline $\mathrm{C}$ & -4.220874 & -1.697916 & 1.517489 \\
\hline $\mathrm{C}$ & -2.036867 & -2.629268 & 1.077747 \\
\hline $\mathrm{C}$ & -2.492413 & -3.885217 & 1.465412 \\
\hline $\mathrm{C}$ & -4.673299 & -2.953740 & 1.905321 \\
\hline $\mathrm{H}$ & -4.900890 & -0.845921 & 1.546011 \\
\hline $\mathrm{H}$ & -0.996527 & -2.494131 & 0.769273 \\
\hline $\mathrm{H}$ & -1.812801 & -4.734921 & 1.452164 \\
\hline $\mathrm{H}$ & -5.703663 & -3.078271 & 2.232376 \\
\hline $\mathrm{H}$ & -4.168362 & -5.028063 & 2.185718 \\
\hline $\mathrm{C}$ & -3.005795 & 1.337320 & 1.623281 \\
\hline $\mathrm{C}$ & -4.066337 & 3.275512 & 3.335750 \\
\hline $\mathrm{C}$ & -3.317612 & 1.016541 & 2.949702 \\
\hline $\mathrm{C}$ & -3.216987 & 2.642998 & 1.167664 \\
\hline $\mathrm{C}$ & -3.740642 & 3.605686 & 2.024247 \\
\hline $\mathrm{C}$ & -3.855795 & 1.978586 & 3.796184 \\
\hline $\mathrm{H}$ & -3.146005 & 0.008397 & 3.324053 \\
\hline $\mathrm{H}$ & -2.963433 & 2.917761 & 0.145554 \\
\hline $\mathrm{H}$ & -3.901146 & 4.618361 & 1.659730 \\
\hline $\mathrm{H}$ & -4.106775 & 1.712470 & 4.821485 \\
\hline $\mathrm{H}$ & -4.483945 & 4.029173 & 4.000423 \\
\hline $\mathrm{C}$ & 0.356386 & 1.508165 & -2.909495 \\
\hline $\mathrm{C}$ & 0.416370 & 4.183550 & -3.775525 \\
\hline $\mathrm{C}$ & -0.569632 & 2.450226 & -2.440240 \\
\hline $\mathrm{C}$ & 1.298457 & 1.942315 & -3.849496 \\
\hline $\mathrm{C}$ & 1.326480 & 3.262990 & -4.284545 \\
\hline $\mathrm{C}$ & -0.539924 & 3.774606 & -2.852553 \\
\hline $\mathrm{H}$ & 2.031710 & 1.234919 & -4.235561 \\
\hline $\mathrm{H}$ & 2.068897 & 3.573669 & -5.016408 \\
\hline $\mathrm{H}$ & -1.275104 & 4.466031 & -2.445010 \\
\hline $\mathrm{H}$ & 0.442829 & 5.220977 & -4.102127 \\
\hline $\mathrm{C}$ & 2.034304 & -0.800658 & -2.687421 \\
\hline $\mathrm{C}$ & 4.616407 & -1.682797 & -3.292540 \\
\hline $\mathrm{C}$ & 3.150285 & -0.164572 & -2.125160 \\
\hline $\mathrm{C}$ & 2.225263 & -1.887456 & -3.542367 \\
\hline $\mathrm{C}$ & 3.513686 & -2.329139 & -3.836620 \\
\hline $\mathrm{C}$ & 4.432416 & -0.595899 & -2.439122 \\
\hline $\mathrm{H}$ & 3.018448 & 0.683856 & -1.449157 \\
\hline $\mathrm{H}$ & 1.368937 & -2.393710 & -3.986285 \\
\hline $\mathrm{H}$ & 3.651013 & -3.178805 & -4.502640 \\
\hline $\mathrm{H}$ & 5.293775 & -0.078218 & -2.019711 \\
\hline $\mathrm{H}$ & 5.621966 & -2.022643 & -3.532696 \\
\hline $\mathrm{C}$ & -0.039648 & 0.439642 & 2.204375 \\
\hline $\mathrm{C}$ & -0.223855 & -0.372578 & 3.245166 \\
\hline $\mathrm{H}$ & -0.493877 & -1.422154 & 3.098996 \\
\hline $\mathrm{C}$ & 0.281819 & 1.903222 & 2.304711 \\
\hline $\mathrm{H}$ & 1.347395 & 2.049512 & 2.058461 \\
\hline
\end{tabular}

\begin{tabular}{|c|c|c|c|}
\hline $\mathrm{H}$ & -0.284932 & 2.477395 & 1.552355 \\
\hline $\mathrm{C}$ & -0.022484 & 2.439595 & 3.708463 \\
\hline $\mathrm{H}$ & -1.112323 & 2.543621 & 3.825193 \\
\hline $\mathrm{H}$ & 0.408178 & 3.443744 & 3.819811 \\
\hline $\mathrm{C}$ & 0.516230 & 1.497010 & 4.774612 \\
\hline C & -0.118372 & 0.113951 & 4.669826 \\
\hline $\mathrm{H}$ & 0.440237 & -0.617125 & 5.274021 \\
\hline $\mathrm{H}$ & -1.135184 & 0.134688 & 5.102620 \\
\hline O & -1.532679 & 2.080303 & -1.519020 \\
\hline 0 & 3.155139 & 0.467409 & 1.255189 \\
\hline B & 2.592981 & -0.752849 & 1.076412 \\
\hline O & 3.140553 & -1.767974 & 1.774726 \\
\hline $\mathrm{C}$ & 4.071053 & -1.149462 & 2.710374 \\
\hline C & 4.365350 & 0.229967 & 2.033728 \\
\hline $\mathrm{H}$ & 1.727031 & -0.975752 & 0.253317 \\
\hline C & 5.506319 & 0.175357 & 1.032396 \\
\hline $\mathrm{H}$ & 5.381622 & -0.660683 & 0.330946 \\
\hline $\mathrm{H}$ & 6.477732 & 0.070773 & 1.531078 \\
\hline $\mathrm{H}$ & 5.516603 & 1.108931 & 0.454920 \\
\hline $\mathrm{C}$ & 4.553769 & 1.385665 & 2.990807 \\
\hline $\mathrm{H}$ & 3.667607 & 1.547346 & 3.615550 \\
\hline $\mathrm{H}$ & 4.742725 & 2.306978 & 2.426424 \\
\hline $\mathrm{H}$ & 5.416062 & 1.208220 & 3.647830 \\
\hline $\mathrm{C}$ & 5.274748 & -2.052181 & 2.864807 \\
\hline $\mathrm{H}$ & 5.736619 & -2.291702 & 1.901585 \\
\hline $\mathrm{H}$ & 4.970925 & -2.993887 & 3.337073 \\
\hline $\mathrm{H}$ & 6.028999 & -1.579421 & 3.508813 \\
\hline $\mathrm{C}$ & 3.324194 & -1.027385 & 4.025651 \\
\hline $\mathrm{H}$ & 2.440551 & -0.387801 & 3.912229 \\
\hline $\mathrm{H}$ & 3.960870 & -0.616999 & 4.819495 \\
\hline $\mathrm{H}$ & 2.982886 & -2.022931 & 4.335274 \\
\hline $\mathrm{H}$ & 1.609711 & 1.410579 & 4.656179 \\
\hline $\mathrm{H}$ & 0.350111 & 1.910056 & 5.778506 \\
\hline
\end{tabular}

SCF energy: -2917.524090070

M06-D3 (Gas Phase) /6-31G(d)+LANL2DZ (Pd) opt. No imaginary frequency 


\begin{tabular}{|c|c|c|c|}
\hline \multicolumn{4}{|c|}{ TS-S2 } \\
\hline $\mathrm{Pd}$ & 0.214296 & 0.594396 & 0.143475 \\
\hline $\mathrm{P}$ & 0.518715 & 0.256180 & -2.214077 \\
\hline $\mathrm{P}$ & -2.113613 & 0.306030 & 0.352418 \\
\hline $\mathrm{C}$ & -0.415703 & -1.146977 & -2.896517 \\
\hline $\mathrm{C}$ & -1.903088 & -3.339144 & -3.759943 \\
\hline $\mathrm{C}$ & -1.076305 & -1.100778 & -4.124335 \\
\hline $\mathrm{C}$ & -0.484607 & -2.305747 & -2.114379 \\
\hline $\mathrm{C}$ & -1.224171 & -3.399128 & -2.544682 \\
\hline $\mathrm{C}$ & -1.824188 & -2.195470 & -4.548508 \\
\hline $\mathrm{H}$ & -1.019676 & -0.205910 & -4.744088 \\
\hline $\mathrm{H}$ & 0.048989 & -2.345641 & -1.162549 \\
\hline $\mathrm{H}$ & -1.280451 & -4.291862 & -1.924108 \\
\hline $\mathrm{H}$ & -2.350295 & -2.151429 & -5.500518 \\
\hline $\mathrm{H}$ & -2.494569 & -4.189265 & -4.094615 \\
\hline $\mathrm{C}$ & -3.048745 & 0.311881 & -1.227227 \\
\hline $\mathrm{C}$ & -4.372593 & 0.326410 & -3.701815 \\
\hline $\mathrm{C}$ & -2.750123 & 1.268835 & -2.204465 \\
\hline $\mathrm{C}$ & -4.000594 & -0.663798 & -1.542685 \\
\hline $\mathrm{C}$ & -4.659008 & -0.660473 & -2.765937 \\
\hline $\mathrm{C}$ & -3.413173 & 1.292070 & -3.425875 \\
\hline $\mathrm{H}$ & -4.215959 & -1.456080 & -0.829589 \\
\hline $\mathrm{H}$ & -5.392599 & -1.432773 & -2.985756 \\
\hline $\mathrm{H}$ & -3.165826 & 2.056830 & -4.159515 \\
\hline $\mathrm{H}$ & -4.890271 & 0.343942 & -4.658930 \\
\hline $\mathrm{C}$ & -2.488326 & -1.306118 & 1.117485 \\
\hline $\mathrm{C}$ & -3.004556 & -3.823030 & 2.218448 \\
\hline $\mathrm{C}$ & -3.776211 & -1.634969 & 1.561284 \\
\hline $\mathrm{C}$ & -1.460911 & -2.245772 & 1.250023 \\
\hline $\mathrm{C}$ & -1.719650 & -3.499905 & 1.794793 \\
\hline $\mathrm{C}$ & -4.031165 & -2.888074 & 2.106382 \\
\hline $\mathrm{H}$ & -4.583220 & -0.905152 & 1.484626 \\
\hline $\mathrm{H}$ & -0.442845 & -1.985566 & 0.952198 \\
\hline $\mathrm{H}$ & -0.910661 & -4.221764 & 1.894063 \\
\hline $\mathrm{H}$ & -5.034844 & -3.134375 & 2.447088 \\
\hline $\mathrm{H}$ & -3.207594 & -4.802860 & 2.646470 \\
\hline $\mathrm{C}$ & -2.901317 & 1.567043 & 1.417505 \\
\hline $\mathrm{C}$ & -3.925210 & 3.567395 & 3.082501 \\
\hline $\mathrm{C}$ & -3.183931 & 1.295463 & 2.760363 \\
\hline $\mathrm{C}$ & -3.121337 & 2.856447 & 0.920334 \\
\hline $\mathrm{C}$ & -3.630734 & 3.848050 & 1.751441 \\
\hline $\mathrm{C}$ & -3.700989 & 2.289386 & 3.584581 \\
\hline $\mathrm{H}$ & -3.006084 & 0.301516 & 3.168968 \\
\hline $\mathrm{H}$ & -2.883117 & 3.094581 & -0.114860 \\
\hline $\mathrm{H}$ & -3.801278 & 4.846579 & 1.353568 \\
\hline $\mathrm{H}$ & -3.927227 & 2.060984 & 4.624942 \\
\hline $\mathrm{H}$ & -4.327948 & 4.345138 & 3.728347 \\
\hline $\mathrm{C}$ & 0.159961 & 1.719057 & -3.249925 \\
\hline $\mathrm{C}$ & -0.282649 & 4.119249 & -4.633199 \\
\hline $\mathrm{C}$ & -0.915654 & 2.556913 & -2.925530 \\
\hline $\mathrm{C}$ & 0.988864 & 2.097796 & -4.312005 \\
\hline $\mathrm{C}$ & 0.765279 & 3.282492 & -5.004826 \\
\hline $\mathrm{C}$ & -1.129507 & 3.755032 & -3.591584 \\
\hline $\mathrm{H}$ & 1.829796 & 1.461755 & -4.586436 \\
\hline $\mathrm{H}$ & 1.420260 & 3.557776 & -5.828392 \\
\hline $\mathrm{H}$ & -1.965839 & 4.382273 & -3.288029 \\
\hline $\mathrm{H}$ & -0.448843 & 5.056351 & -5.160298 \\
\hline $\mathrm{C}$ & 2.278955 & -0.110986 & -2.552391 \\
\hline $\mathrm{C}$ & 4.997100 & -0.627821 & -2.928842 \\
\hline $\mathrm{C}$ & 3.236825 & 0.854723 & -2.214649 \\
\hline $\mathrm{C}$ & 2.694275 & -1.336604 & -3.073093 \\
\hline $\mathrm{C}$ & 4.052752 & -1.594463 & -3.251067 \\
\hline $\mathrm{C}$ & 4.586378 & 0.602482 & -2.415137 \\
\hline $\mathrm{H}$ & 2.923004 & 1.813483 & -1.796780 \\
\hline $\mathrm{H}$ & 1.960666 & -2.094304 & -3.345059 \\
\hline $\mathrm{H}$ & 4.368497 & -2.555014 & -3.653618 \\
\hline $\mathrm{H}$ & 5.322421 & 1.362114 & -2.157925 \\
\hline $\mathrm{H}$ & 6.056435 & -0.829911 & -3.074766 \\
\hline $\mathrm{C}$ & 0.335005 & 1.028050 & 2.201738 \\
\hline $\mathrm{C}$ & -0.004191 & 0.217193 & 3.217278 \\
\hline $\mathrm{H}$ & -0.209129 & -0.839250 & 3.028861 \\
\hline $\mathrm{C}$ & 0.578558 & 2.505476 & 2.374193 \\
\hline $\mathrm{H}$ & 1.643569 & 2.717247 & 2.182553 \\
\hline
\end{tabular}

\begin{tabular}{|c|c|c|c|}
\hline $\mathrm{H}$ & 0.017978 & 3.065785 & 1.606941 \\
\hline $\mathrm{C}$ & 0.182824 & 2.997557 & 3.767628 \\
\hline $\mathrm{H}$ & -0.913573 & 3.101199 & 3.821199 \\
\hline $\mathrm{H}$ & 0.604561 & 3.996357 & 3.939650 \\
\hline $\mathrm{C}$ & 0.648551 & 2.011396 & 4.829610 \\
\hline $\mathrm{C}$ & -0.061348 & 0.677492 & 4.643947 \\
\hline $\mathrm{H}$ & 0.362376 & -0.098771 & 5.299370 \\
\hline $\mathrm{H}$ & -1.122618 & 0.764105 & 4.941059 \\
\hline O & -1.776637 & 2.203224 & -1.903656 \\
\hline 0 & 3.117582 & 0.562831 & 2.132032 \\
\hline B & 2.033058 & 0.080183 & 1.438958 \\
\hline 0 & 2.003191 & -1.297238 & 1.354093 \\
\hline $\mathrm{C}$ & 3.052527 & -1.778180 & 2.233201 \\
\hline $\mathrm{C}$ & 4.022981 & -0.559889 & 2.269520 \\
\hline $\mathrm{H}$ & 1.922362 & 0.782059 & 0.297855 \\
\hline $\mathrm{C}$ & 4.958219 & -0.520970 & 1.069909 \\
\hline $\mathrm{H}$ & 4.411888 & -0.672133 & 0.128116 \\
\hline $\mathrm{H}$ & 5.743377 & -1.284370 & 1.142742 \\
\hline $\mathrm{H}$ & 5.439386 & 0.464160 & 1.027179 \\
\hline C & 4.802027 & -0.399134 & 3.556401 \\
\hline $\mathrm{H}$ & 4.140240 & -0.255048 & 4.417384 \\
\hline $\mathrm{H}$ & 5.457665 & 0.477003 & 3.484912 \\
\hline $\mathrm{H}$ & 5.433194 & -1.279445 & 3.740496 \\
\hline C & 3.629837 & -3.039935 & 1.628930 \\
\hline $\mathrm{H}$ & 3.924225 & -2.889109 & 0.584466 \\
\hline $\mathrm{H}$ & 2.880017 & -3.840987 & 1.657604 \\
\hline $\mathrm{H}$ & 4.506239 & -3.379353 & 2.197929 \\
\hline $\mathrm{C}$ & 2.430512 & -2.079792 & 3.587160 \\
\hline $\mathrm{H}$ & 2.063977 & -1.168801 & 4.077554 \\
\hline $\mathrm{H}$ & 3.151419 & -2.563335 & 4.258369 \\
\hline $\mathrm{H}$ & 1.581788 & -2.763434 & 3.450296 \\
\hline $\mathrm{H}$ & 1.736420 & 1.855749 & 4.731686 \\
\hline $\mathrm{H}$ & 0.469980 & 2.401615 & 5.839656 \\
\hline
\end{tabular}

SCF energy: -2917.517781980

M06-D3 (Gas Phase) /6-31G(d)+LANL2DZ (Pd) opt. 1 imaginary frequency: $151 \mathrm{i} \mathrm{cm-1}$ 


\begin{tabular}{|c|c|c|c|}
\hline \multicolumn{4}{|c|}{ Int-11 } \\
\hline $\mathrm{Pd}$ & 0.147807 & 0.957164 & 0.484658 \\
\hline $\mathrm{P}$ & 0.660438 & 0.686640 & -1.775285 \\
\hline $\mathrm{P}$ & -2.220529 & 0.151708 & 0.538271 \\
\hline $\mathrm{C}$ & -0.029633 & -0.876820 & -2.393877 \\
\hline $\mathrm{C}$ & -0.961536 & -3.364189 & -3.224621 \\
\hline $\mathrm{C}$ & -0.530500 & -1.033132 & -3.686548 \\
\hline $\mathrm{C}$ & 0.018938 & -1.972173 & -1.526298 \\
\hline $\mathrm{C}$ & -0.444048 & -3.214180 & -1.939931 \\
\hline $\mathrm{C}$ & -1.003580 & -2.277355 & -4.094106 \\
\hline $\mathrm{H}$ & -0.558732 & -0.184230 & -4.370619 \\
\hline $\mathrm{H}$ & 0.410258 & -1.840185 & -0.514902 \\
\hline $\mathrm{H}$ & -0.412906 & -4.059186 & -1.252736 \\
\hline $\mathrm{H}$ & -1.409854 & -2.396612 & -5.096870 \\
\hline $\mathrm{H}$ & -1.336188 & -4.332714 & -3.550270 \\
\hline $\mathrm{C}$ & -3.005784 & 0.002090 & -1.122514 \\
\hline $\mathrm{C}$ & -4.070580 & -0.192412 & -3.717048 \\
\hline $\mathrm{C}$ & -2.783235 & 0.998280 & -2.079698 \\
\hline $\mathrm{C}$ & -3.750144 & -1.113896 & -1.518334 \\
\hline $\mathrm{C}$ & -4.277931 & -1.215689 & -2.800310 \\
\hline $\mathrm{C}$ & -3.317687 & 0.919230 & -3.360009 \\
\hline $\mathrm{H}$ & -3.902588 & -1.933243 & -0.819881 \\
\hline $\mathrm{H}$ & -4.850874 & -2.097474 & -3.078583 \\
\hline $\mathrm{H}$ & -3.133782 & 1.718718 & -4.074883 \\
\hline $\mathrm{H}$ & -4.489250 & -0.256977 & -4.719487 \\
\hline $\mathrm{C}$ & -2.555615 & -1.463859 & 1.327650 \\
\hline $\mathrm{C}$ & -3.011887 & -3.951380 & 2.521868 \\
\hline $\mathrm{C}$ & -3.863594 & -1.894482 & 1.596844 \\
\hline $\mathrm{C}$ & -1.483670 & -2.285772 & 1.679984 \\
\hline $\mathrm{C}$ & -1.712995 & -3.524429 & 2.274937 \\
\hline $\mathrm{C}$ & -4.088483 & -3.132030 & 2.185178 \\
\hline $\mathrm{H}$ & -4.710108 & -1.252042 & 1.351796 \\
\hline $\mathrm{H}$ & -0.460653 & -1.945485 & 1.523079 \\
\hline $\mathrm{H}$ & -0.868629 & -4.153094 & 2.557205 \\
\hline $\mathrm{H}$ & -5.107172 & -3.456760 & 2.388105 \\
\hline $\mathrm{H}$ & -3.190584 & -4.918717 & 2.987705 \\
\hline $\mathrm{C}$ & -3.313530 & 1.296577 & 1.474127 \\
\hline $\mathrm{C}$ & -4.779229 & 3.141149 & 2.990250 \\
\hline $\mathrm{C}$ & -3.621699 & 1.033203 & 2.813979 \\
\hline $\mathrm{C}$ & -3.742536 & 2.501362 & 0.905614 \\
\hline $\mathrm{C}$ & -4.471497 & 3.413971 & 1.660812 \\
\hline $\mathrm{C}$ & -4.354502 & 1.947335 & 3.563546 \\
\hline $\mathrm{H}$ & -3.292597 & 0.105783 & 3. 281742 \\
\hline $\mathrm{H}$ & -3.499023 & 2.739508 & -0.127351 \\
\hline $\mathrm{H}$ & -4.803116 & 4.344587 & 1.203864 \\
\hline $\mathrm{H}$ & -4.592791 & 1.722549 & 4.601675 \\
\hline $\mathrm{H}$ & -5.351175 & 3.856983 & 3.577351 \\
\hline $\mathrm{C}$ & 0.087034 & 2.053256 & -2.844934 \\
\hline C & -0.727592 & 4.329424 & -4.266348 \\
\hline $\mathrm{C}$ & -1.170833 & 2.632047 & -2.628750 \\
\hline $\mathrm{C}$ & 0.916956 & 2.626760 & -3.815061 \\
\hline $\mathrm{C}$ & 0.509160 & 3.749125 & -4.527642 \\
\hline $\mathrm{C}$ & -1.573162 & 3.768789 & -3.314422 \\
\hline $\mathrm{H}$ & 1.899451 & 2.197492 & -4.006017 \\
\hline $\mathrm{H}$ & 1.167766 & 4.178228 & -5.279339 \\
\hline $\mathrm{H}$ & -2.550916 & 4.194606 & -3.095784 \\
\hline $\mathrm{H}$ & -1.041117 & 5.219115 & -4.808379 \\
\hline $\mathrm{C}$ & 2.444932 & 0.563890 & -2.170726 \\
\hline $\mathrm{C}$ & 5.158608 & 0.465683 & -2.825641 \\
\hline $\mathrm{C}$ & 3.329030 & 1.528646 & -1.674147 \\
\hline $\mathrm{C}$ & 2.932240 & -0.448210 & -3.000934 \\
\hline $\mathrm{C}$ & 4.288144 & -0.499261 & -3.317992 \\
\hline $\mathrm{C}$ & 4.675613 & 1.485876 & -2.006941 \\
\hline $\mathrm{H}$ & 2.959518 & 2.326666 & -1.026226 \\
\hline $\mathrm{H}$ & 2.258862 & -1.206165 & -3.398691 \\
\hline $\mathrm{H}$ & 4.660556 & -1.297949 & -3.956437 \\
\hline $\mathrm{H}$ & 5.352538 & 2. 244727 & -1.619619 \\
\hline $\mathrm{H}$ & 6.216767 & 0.424637 & -3.076501 \\
\hline $\mathrm{C}$ & 1.374727 & 1.238427 & 2.538909 \\
\hline $\mathrm{C}$ & 0.024890 & 1.290697 & 2.838103 \\
\hline $\mathrm{H}$ & -0.494275 & 0.347913 & 3.033270 \\
\hline $\mathrm{C}$ & 2.221816 & 2.491158 & 2.616544 \\
\hline $\mathrm{H}$ & 2.735014 & 2.460070 & 3.594165 \\
\hline
\end{tabular}

\begin{tabular}{|c|c|c|c|}
\hline $\mathrm{H}$ & 3.027000 & 2.455346 & 1.870296 \\
\hline C & 1.405774 & 3.767615 & 2.515356 \\
\hline $\mathrm{H}$ & 1.005602 & 3.868116 & 1.490286 \\
\hline $\mathrm{H}$ & 2.044900 & 4.641868 & 2.694908 \\
\hline C & 0.259368 & 3.719157 & 3.515049 \\
\hline C & -0.690880 & 2.565627 & 3.209499 \\
\hline $\mathrm{H}$ & -1.346148 & 2.366565 & 4.069703 \\
\hline $\mathrm{H}$ & -1.377268 & 2.852896 & 2.395191 \\
\hline O & -2.016051 & 2.082379 & -1.689050 \\
\hline 0 & 3.493407 & -0.185854 & 2.359593 \\
\hline B & 2.133260 & -0.117534 & 2.351099 \\
\hline O & 1.527313 & -1.333435 & 2.156195 \\
\hline $\mathrm{C}$ & 2.568530 & -2.338169 & 2.256368 \\
\hline C & 3.851161 & -1.517904 & 1.916714 \\
\hline $\mathrm{H}$ & 1.598552 & 1.557530 & 0.303601 \\
\hline C & 4.116915 & -1.415659 & 0.424090 \\
\hline $\mathrm{H}$ & 3.217752 & -1.101750 & -0.125733 \\
\hline $\mathrm{H}$ & 4.468169 & -2.366833 & 0.003982 \\
\hline $\mathrm{H}$ & 4.890909 & -0.657656 & 0.252008 \\
\hline C & 5.100275 & -1.960402 & 2.646496 \\
\hline $\mathrm{H}$ & 4.986932 & -1.887533 & 3.733055 \\
\hline $\mathrm{H}$ & 5.944857 & -1.326030 & 2.352104 \\
\hline $\mathrm{H}$ & 5.351351 & -2.998473 & 2.388352 \\
\hline $\mathrm{C}$ & 2.258972 & -3.461510 & 1.288740 \\
\hline $\mathrm{H}$ & 2.159367 & -3.104509 & 0.256894 \\
\hline $\mathrm{H}$ & 1.317397 & -3.951761 & 1.573381 \\
\hline $\mathrm{H}$ & 3.050478 & -4.222752 & 1.314004 \\
\hline C & 2.534471 & -2.851137 & 3.686733 \\
\hline $\mathrm{H}$ & 2.762202 & -2.053626 & 4.405476 \\
\hline $\mathrm{H}$ & 3.245309 & -3.672210 & 3.841650 \\
\hline $\mathrm{H}$ & 1.525639 & -3.223466 & 3.906725 \\
\hline $\mathrm{H}$ & 0.679563 & 3.591176 & 4.525718 \\
\hline $\mathrm{H}$ & -0.299497 & 4.663462 & 3.527875 \\
\hline
\end{tabular}

SCF energy: -2917.558755330

M06-D3 (Gas Phase) /6-31G(d)+LANL2DZ (Pd) opt. No imaginary frequency 


\begin{tabular}{|c|c|c|c|}
\hline \multicolumn{4}{|l|}{ TS-8 } \\
\hline $\mathrm{Pd}$ & 0.869439 & 1.267256 & 1.206712 \\
\hline $\mathrm{P}$ & 1.715127 & 0.987685 & -1.356446 \\
\hline $\mathrm{P}$ & -1.468556 & 0.977736 & 0.888964 \\
\hline $\mathrm{C}$ & 0.757826 & -0.387163 & -2.086659 \\
\hline $\mathrm{C}$ & -0.565176 & -2.644942 & -3.060683 \\
\hline $\mathrm{C}$ & 0.235593 & -0.388671 & -3.379939 \\
\hline $\mathrm{C}$ & 0.633995 & -1.533546 & -1.295291 \\
\hline $\mathrm{C}$ & -0.017609 & -2.661408 & -1.780466 \\
\hline $\mathrm{C}$ & -0.436633 & -1.510191 & -3.857469 \\
\hline $\mathrm{H}$ & 0.351081 & 0.490525 & -4.014685 \\
\hline $\mathrm{H}$ & 1.059396 & -1.536865 & -0.287337 \\
\hline $\mathrm{H}$ & -0.106217 & -3.548541 & -1.152470 \\
\hline $\mathrm{H}$ & -0.858513 & -1.498836 & -4.861177 \\
\hline $\mathrm{H}$ & -1.088392 & -3.520106 & -3.441360 \\
\hline $\mathrm{C}$ & -2.072834 & 0.948799 & -0.847680 \\
\hline $\mathrm{C}$ & -3.014760 & 1.098104 & -3.481810 \\
\hline $\mathrm{C}$ & -1.607411 & 1.931583 & -1.726492 \\
\hline $\mathrm{C}$ & -3.015539 & 0.033375 & -1.324701 \\
\hline $\mathrm{C}$ & -3.480649 & 0.100529 & -2.633675 \\
\hline $\mathrm{C}$ & -2.076425 & 2.019545 & -3.031984 \\
\hline $\mathrm{H}$ & -3.393851 & -0.746157 & -0.665826 \\
\hline $\mathrm{H}$ & -4.211018 & -0.625096 & -2.984754 \\
\hline $\mathrm{H}$ & -1.701467 & 2.798500 & -3.692724 \\
\hline $\mathrm{H}$ & -3.381488 & 1.165023 & -4.504429 \\
\hline $\mathrm{C}$ & -2.384040 & -0.460030 & 1.564164 \\
\hline $\mathrm{C}$ & -3.802300 & -2.748768 & 2.317181 \\
\hline $\mathrm{C}$ & -3.583656 & -0.344925 & 2.271664 \\
\hline $\mathrm{C}$ & -1.897320 & -1.731872 & 1.250363 \\
\hline $\mathrm{C}$ & -2.612040 & -2.868647 & 1.604312 \\
\hline $\mathrm{C}$ & -4.279058 & -1.487365 & 2.660508 \\
\hline $\mathrm{H}$ & -3.982164 & 0.639258 & 2.518262 \\
\hline $\mathrm{H}$ & -0.960148 & -1.828412 & 0.704000 \\
\hline $\mathrm{H}$ & -2.228184 & -3.851674 & 1.334849 \\
\hline $\mathrm{H}$ & -5.209011 & -1.387636 & 3.217394 \\
\hline $\mathrm{H}$ & -4.359285 & -3.638662 & 2.604875 \\
\hline $\mathrm{C}$ & -2.361865 & 2.441769 & 1.541750 \\
\hline $\mathrm{C}$ & -3.699181 & 4.657442 & 2.603381 \\
\hline $\mathrm{C}$ & -1.777220 & 3.209514 & 2.548849 \\
\hline $\mathrm{C}$ & -3.623152 & 2.806699 & 1.056518 \\
\hline $\mathrm{C}$ & -4.286689 & 3.906950 & 1.587605 \\
\hline $\mathrm{C}$ & -2.441442 & 4.308637 & 3.083858 \\
\hline $\mathrm{H}$ & -0.778675 & 2.941136 & 2.898907 \\
\hline $\mathrm{H}$ & -4.089387 & 2.227275 & 0.258944 \\
\hline $\mathrm{H}$ & -5.267457 & 4.181642 & 1.203891 \\
\hline $\mathrm{H}$ & -1.968838 & 4.899346 & 3.867809 \\
\hline $\mathrm{H}$ & -4.220617 & 5.519827 & 3.014331 \\
\hline $\mathrm{C}$ & 1.389199 & 2.487434 & -2.387891 \\
\hline $\mathrm{C}$ & 1.022907 & 5.014838 & -3.586437 \\
\hline $\mathrm{C}$ & 0.269655 & 3.283641 & -2.106217 \\
\hline $\mathrm{C}$ & 2.295488 & 2.981110 & -3.334413 \\
\hline $\mathrm{C}$ & 2.113482 & 4.224333 & -3.932325 \\
\hline $\mathrm{C}$ & 0.089858 & 4.537792 & -2.671984 \\
\hline $\mathrm{H}$ & 3.169804 & 2.388722 & -3.597820 \\
\hline $\mathrm{H}$ & 2.835714 & 4.578375 & -4.664976 \\
\hline $\mathrm{H}$ & -0.791043 & 5.114195 & -2.393522 \\
\hline $\mathrm{H}$ & 0.888283 & 5.996110 & -4.036718 \\
\hline $\mathrm{C}$ & 3.421458 & 0.544130 & -1.907262 \\
\hline $\mathrm{C}$ & 6.043076 & -0.079021 & -2.697412 \\
\hline $\mathrm{C}$ & 4.485992 & 1.405086 & -1.608795 \\
\hline $\mathrm{C}$ & 3.696445 & -0.638289 & -2.602674 \\
\hline $\mathrm{C}$ & 4.998600 & -0.949437 & -2.987071 \\
\hline $\mathrm{C}$ & 5.780723 & 1.104643 & -2.010882 \\
\hline $\mathrm{H}$ & 4.309281 & 2.335789 & -1.072077 \\
\hline $\mathrm{H}$ & 2.892261 & -1.327079 & -2.855612 \\
\hline $\mathrm{H}$ & 5.190827 & -1.874164 & -3.528148 \\
\hline $\mathrm{H}$ & 6.589437 & 1.795541 & -1.777791 \\
\hline $\mathrm{H}$ & 7.058473 & -0.317973 & -3.008442 \\
\hline $\mathrm{C}$ & 2.680914 & 2.565385 & 2.381704 \\
\hline $\mathrm{C}$ & 2.476193 & 3.201883 & 1.188834 \\
\hline $\mathrm{H}$ & 2.988198 & 2.810272 & 0.311076 \\
\hline $\mathrm{C}$ & 2.236653 & 3.262005 & 3.658925 \\
\hline $\mathrm{H}$ & 1.228686 & 2.936366 & 3.962013 \\
\hline
\end{tabular}

\begin{tabular}{|c|c|c|c|}
\hline $\mathrm{H}$ & 2.904923 & 2.971364 & 4.482483 \\
\hline C & 2.223186 & 4.776345 & 3.476379 \\
\hline $\mathrm{H}$ & 3.249905 & 5.128542 & 3.284321 \\
\hline $\mathrm{H}$ & 1.888815 & 5.266545 & 4.400509 \\
\hline C & 1.320492 & 5.158559 & 2.311604 \\
\hline C & 1.795553 & 4.520425 & 1.006143 \\
\hline $\mathrm{H}$ & 0.961297 & 4.412531 & 0.293506 \\
\hline $\mathrm{H}$ & 2.518840 & 5.179157 & 0.494639 \\
\hline O & -0.685473 & 2.821384 & -1.220827 \\
\hline 0 & 3.844428 & 0.573774 & 3.526958 \\
\hline B & 3.698265 & 1.385163 & 2.431674 \\
\hline O & 4.582517 & 1.091202 & 1.430340 \\
\hline C & 5.237464 & -0.150277 & 1.785675 \\
\hline C & 5.060714 & -0.188364 & 3.336331 \\
\hline $\mathrm{H}$ & 0.390793 & 0.926111 & 2.835719 \\
\hline C & 6.154458 & 0.557001 & 4.085381 \\
\hline $\mathrm{H}$ & 6.312242 & 1.561567 & 3.671901 \\
\hline $\mathrm{H}$ & 7.108146 & 0.015666 & 4.057209 \\
\hline $\mathrm{H}$ & 5.854724 & 0.666579 & 5.134608 \\
\hline C & 4.865176 & -1.567386 & 3.929648 \\
\hline $\mathrm{H}$ & 3.972940 & -2.063908 & 3.530656 \\
\hline $\mathrm{H}$ & 4.746996 & -1.488573 & 5.017925 \\
\hline $\mathrm{H}$ & 5.738887 & -2.203468 & 3.732529 \\
\hline C & 6.679441 & -0.103885 & 1.325251 \\
\hline $\mathrm{H}$ & 7.194477 & 0.792771 & 1.685081 \\
\hline $\mathrm{H}$ & 6.718743 & -0.103721 & 0.229313 \\
\hline $\mathrm{H}$ & 7.224891 & -0.988342 & 1.682788 \\
\hline C & 4.502683 & -1.266678 & 1.058022 \\
\hline $\mathrm{H}$ & 3.435990 & -1.282601 & 1.320607 \\
\hline $\mathrm{H}$ & 4.934851 & -2.250201 & 1.283107 \\
\hline $\mathrm{H}$ & 4.572554 & -1.100220 & -0.023885 \\
\hline $\mathrm{N}$ & 0.139333 & 0.160576 & 4.213106 \\
\hline C & 1.189599 & 0.435579 & 5.214536 \\
\hline $\mathrm{H}$ & 2.178462 & 0.225078 & 4.799833 \\
\hline $\mathrm{H}$ & 1.016188 & -0.192105 & 6.102927 \\
\hline $\mathrm{H}$ & 1.140510 & 1.488853 & 5.509426 \\
\hline C & -1.149549 & 0.615447 & 4.740321 \\
\hline $\mathrm{H}$ & -1.961043 & 0.316384 & 4.071202 \\
\hline $\mathrm{H}$ & -1.132421 & 1.708158 & 4.819068 \\
\hline $\mathrm{H}$ & -1.336463 & 0.204079 & 5.744096 \\
\hline C & 0.186229 & -1.237567 & 3.841605 \\
\hline C & 0.453931 & -3.924601 & 3.138522 \\
\hline C & -0.672747 & -2.186983 & 4.387050 \\
\hline C & 1.183584 & -1.635136 & 2.951203 \\
\hline C & 1.312652 & -2.971434 & 2.594426 \\
\hline C & -0.533070 & -3.526802 & 4.032751 \\
\hline $\mathrm{H}$ & -1.462005 & -1.896999 & 5.077661 \\
\hline $\mathrm{H}$ & 1.862668 & -0.876339 & 2.557095 \\
\hline $\mathrm{H}$ & 2.093650 & -3.269896 & 1.894109 \\
\hline $\mathrm{H}$ & -1.211181 & -4.262999 & 4.461068 \\
\hline $\mathrm{H}$ & 0.557518 & -4.973595 & 2.867828 \\
\hline $\mathrm{H}$ & 0.293321 & 4.831210 & 2.530007 \\
\hline $\mathrm{H}$ & 1.272748 & 6.248681 & 2.192770 \\
\hline
\end{tabular}

SCF energy: -3283.488755110

M06-D3 (Gas Phase) /6-31G(d) +LANL2DZ (Pd) opt. 1 imaginary frequency: $646 \mathrm{i} \mathrm{cm-1}$ 


\begin{tabular}{|c|c|c|c|}
\hline \multicolumn{4}{|c|}{ Int-15 } \\
\hline $\mathrm{Pd}$ & -0.013923 & 0.840990 & 0.667677 \\
\hline $\mathrm{P}$ & 0.690568 & 0.760039 & -1.603933 \\
\hline $\mathrm{P}$ & -2.369529 & 0.240608 & 0.551507 \\
\hline $\mathrm{C}$ & 0.180246 & -0.830238 & -2.371726 \\
\hline $\mathrm{C}$ & -0.572961 & -3.338276 & -3.353081 \\
\hline $\mathrm{C}$ & -0.099100 & -1.001165 & -3.728960 \\
\hline $\mathrm{C}$ & 0.079735 & -1.930162 & -1.513234 \\
\hline $\mathrm{C}$ & -0.293005 & -3.178163 & -1.998741 \\
\hline $\mathrm{C}$ & -0.474959 & -2.250032 & -4.215792 \\
\hline $\mathrm{H}$ & -0.032459 & -0.148601 & -4.407555 \\
\hline $\mathrm{H}$ & 0.276741 & -1.786941 & -0.447227 \\
\hline $\mathrm{H}$ & -0.383825 & -4.018832 & -1.310437 \\
\hline $\mathrm{H}$ & -0.702619 & -2.371020 & -5.274186 \\
\hline $\mathrm{H}$ & -0.877689 & -4.310838 & -3.736874 \\
\hline $\mathrm{C}$ & -3.034191 & -0.138849 & -1.124988 \\
\hline $\mathrm{C}$ & -3.760030 & -0.687701 & -3.783330 \\
\hline $\mathrm{C}$ & -2.756129 & 0.764311 & -2.159211 \\
\hline $\mathrm{C}$ & -3.674152 & -1.332908 & -1.466531 \\
\hline $\mathrm{C}$ & -4.034296 & -1.611099 & -2.781305 \\
\hline $\mathrm{C}$ & -3.121325 & 0.507747 & -3.476018 \\
\hline $\mathrm{H}$ & -3.874219 & -2.070267 & -0.690696 \\
\hline $\mathrm{H}$ & -4.522762 & -2.553906 & -3.020441 \\
\hline $\mathrm{H}$ & -2.888306 & 1.233425 & -4.253387 \\
\hline $\mathrm{H}$ & -4.036874 & -0.897060 & -4.815525 \\
\hline $\mathrm{C}$ & -2.764205 & -1.301056 & 1.476742 \\
\hline $\mathrm{C}$ & -3.249670 & -3.672966 & 2.880306 \\
\hline $\mathrm{C}$ & -4.070047 & -1.662551 & 1.830141 \\
\hline $\mathrm{C}$ & -1.706120 & -2.136693 & 1.843783 \\
\hline $\mathrm{C}$ & -1.949015 & -3.316650 & 2.542232 \\
\hline $\mathrm{C}$ & -4.311138 & -2.842610 & 2.523987 \\
\hline $\mathrm{H}$ & -4.902255 & -1.010729 & 1.559249 \\
\hline $\mathrm{H}$ & -0.679278 & -1.846112 & 1.614069 \\
\hline $\mathrm{H}$ & -1.110431 & -3.947955 & 2.837218 \\
\hline $\mathrm{H}$ & -5.330993 & -3.114273 & 2.793071 \\
\hline $\mathrm{H}$ & -3.440180 & -4.593095 & 3.431137 \\
\hline $\mathrm{C}$ & -3.631434 & 1.387240 & 1. 240597 \\
\hline $\mathrm{C}$ & -5.407814 & 3.204457 & 2.421102 \\
\hline $\mathrm{C}$ & -3.616399 & 1.606290 & 2.623863 \\
\hline $\mathrm{C}$ & -4.551604 & 2.087941 & 0.459153 \\
\hline $\mathrm{C}$ & -5.431977 & 2.992528 & 1.047152 \\
\hline $\mathrm{C}$ & -4.498456 & 2.503790 & 3.210532 \\
\hline $\mathrm{H}$ & -2.911612 & 1.053967 & 3.248018 \\
\hline $\mathrm{H}$ & -4.588994 & 1.921616 & -0.616738 \\
\hline $\mathrm{H}$ & -6.145919 & 3.529966 & 0.424287 \\
\hline $\mathrm{H}$ & -4.474914 & 2.657957 & 4.288457 \\
\hline $\mathrm{H}$ & -6.097797 & 3.912089 & 2.878221 \\
\hline $\mathrm{C}$ & 0.068162 & 2.050560 & -2.779296 \\
\hline C & -0.857923 & 4.210974 & -4.342087 \\
\hline $\mathrm{C}$ & -1.254080 & 2.511777 & -2.687414 \\
\hline $\mathrm{C}$ & 0.903439 & 2.697357 & -3.698175 \\
\hline $\mathrm{C}$ & 0.449701 & 3.758608 & -4.475353 \\
\hline $\mathrm{C}$ & -1.712900 & 3.582340 & -3.444124 \\
\hline $\mathrm{H}$ & 1.937703 & 2.370647 & -3.798810 \\
\hline $\mathrm{H}$ & 1.127345 & 4.237975 & -5.179592 \\
\hline $\mathrm{H}$ & -2.744531 & 3.904462 & -3.311073 \\
\hline $\mathrm{H}$ & -1.215923 & 5.050143 & -4.935841 \\
\hline $\mathrm{C}$ & 2.489311 & 0.805449 & -1.991759 \\
\hline $\mathrm{C}$ & 5.249122 & 0.999271 & -2.409542 \\
\hline $\mathrm{C}$ & 3.253187 & 1.737411 & -1.283869 \\
\hline $\mathrm{C}$ & 3.122724 & -0.025479 & -2.918690 \\
\hline $\mathrm{C}$ & 4.496737 & 0.071080 & -3.123633 \\
\hline $\mathrm{C}$ & 4.623420 & 1.838540 & -1.490311 \\
\hline $\mathrm{H}$ & 2.754770 & 2.389906 & -0.564956 \\
\hline $\mathrm{H}$ & 2.543196 & -0.762317 & -3.475295 \\
\hline $\mathrm{H}$ & 4.982003 & -0.586761 & -3.843504 \\
\hline $\mathrm{H}$ & 5.204312 & 2.565845 & -0.925039 \\
\hline $\mathrm{H}$ & 6.324424 & 1.067539 & -2.567647 \\
\hline $\mathrm{C}$ & 1.556945 & 0.970791 & 2.335765 \\
\hline $\mathrm{C}$ & 0.270986 & 1.050562 & 2.879313 \\
\hline $\mathrm{H}$ & -0.205872 & 0.125167 & 3.215866 \\
\hline $\mathrm{C}$ & 2.446549 & 2.194988 & 2.295503 \\
\hline $\mathrm{H}$ & 3.134300 & 2.154809 & 3.161406 \\
\hline
\end{tabular}

\begin{tabular}{|c|c|c|c|}
\hline $\mathrm{H}$ & 3.102765 & 2.162074 & 1.414563 \\
\hline C & 1.658012 & 3.492709 & 2.360445 \\
\hline $\mathrm{H}$ & 1.068559 & 3.611582 & 1.432929 \\
\hline $\mathrm{H}$ & 2.337427 & 4.355116 & 2.425705 \\
\hline C & 0.722190 & 3.453770 & 3.561465 \\
\hline C & -0.312603 & 2.340075 & 3.422072 \\
\hline $\mathrm{H}$ & -0.786512 & 2.147111 & 4.398441 \\
\hline $\mathrm{H}$ & -1.129328 & 2.686961 & 2.766490 \\
\hline O & -2.129901 & 1.933884 & -1.794755 \\
\hline O & 3.531383 & -0.556303 & 1.748516 \\
\hline B & 2.191643 & -0.397130 & 2.027014 \\
\hline O & 1.524962 & -1.615273 & 2.053763 \\
\hline C & 2.521726 & -2.647638 & 2.026559 \\
\hline C & 3.738952 & -1.925580 & 1.368542 \\
\hline C & 3.719259 & -1.979646 & -0.151020 \\
\hline $\mathrm{H}$ & 2.746977 & -1.667500 & -0.556116 \\
\hline $\mathrm{H}$ & 3.946399 & -2.985771 & -0.528722 \\
\hline $\mathrm{H}$ & 4.474108 & -1.283931 & -0.539236 \\
\hline C & 5.093830 & -2.373805 & 1.879254 \\
\hline $\mathrm{H}$ & 5.200219 & -2.201939 & 2.955802 \\
\hline $\mathrm{H}$ & 5.882987 & -1.808168 & 1.368287 \\
\hline $\mathrm{H}$ & 5.254845 & -3.442081 & 1.675051 \\
\hline C & 1.983209 & -3.828621 & 1.243401 \\
\hline $\mathrm{H}$ & 1.688586 & -3.543899 & 0.226853 \\
\hline $\mathrm{H}$ & 1.098576 & -4.236928 & 1.751540 \\
\hline $\mathrm{H}$ & 2.733140 & -4.629481 & 1.175722 \\
\hline C & 2.769333 & -3.053257 & 3.473067 \\
\hline $\mathrm{H}$ & 3.160131 & -2.211234 & 4.059031 \\
\hline $\mathrm{H}$ & 3.472458 & -3.892787 & 3.552595 \\
\hline $\mathrm{H}$ & 1.813837 & -3.357384 & 3.919966 \\
\hline $\mathrm{H}$ & 1.329593 & 3.278659 & 4.465711 \\
\hline $\mathrm{H}$ & 0.215979 & 4.418719 & 3.705449 \\
\hline
\end{tabular}

SCF energy: -2917.165434120

M06-D3 (Gas Phase)/6-31G(d) +LANL2DZ (Pd) opt. No imaginary frequency 


\begin{tabular}{|c|c|c|c|}
\hline \multicolumn{4}{|l|}{ TS-9 } \\
\hline $\mathrm{Pd}$ & 0.028376 & 0.919600 & 0.525327 \\
\hline $\mathrm{P}$ & 0.595977 & 0.715517 & -1.754379 \\
\hline $\mathrm{P}$ & -2.308887 & 0.208442 & 0.521094 \\
\hline $\mathrm{C}$ & -0.014330 & -0.871522 & -2.402354 \\
\hline $\mathrm{C}$ & -0.918199 & -3.376504 & -3.220143 \\
\hline $\mathrm{C}$ & -0.514054 & -1.042624 & -3.693232 \\
\hline $\mathrm{C}$ & 0.048516 & -1.964199 & -1.530809 \\
\hline $\mathrm{C}$ & -0.399681 & -3.213737 & -1.937231 \\
\hline $\mathrm{C}$ & -0.971742 & -2.294939 & -4.094908 \\
\hline $\mathrm{H}$ & -0.557998 & -0.196938 & -4.380407 \\
\hline $\mathrm{H}$ & 0.437071 & -1.823820 & -0.518883 \\
\hline $\mathrm{H}$ & -0.359294 & -4.053493 & -1.244003 \\
\hline $\mathrm{H}$ & -1.377333 & -2.423755 & -5.096872 \\
\hline $\mathrm{H}$ & -1.284020 & -4.350460 & -3.539688 \\
\hline $\mathrm{C}$ & -3.064639 & 0.004566 & -1.143581 \\
\hline $\mathrm{C}$ & -4.060880 & -0.291764 & -3.753441 \\
\hline $\mathrm{C}$ & -2.837694 & 0.976185 & -2.124902 \\
\hline $\mathrm{C}$ & -3.773016 & -1.140793 & -1.521475 \\
\hline $\mathrm{C}$ & -4.268208 & -1.292166 & -2.811308 \\
\hline $\mathrm{C}$ & -3.340045 & 0.846242 & -3.414109 \\
\hline $\mathrm{H}$ & -3.922376 & -1.941945 & -0.801176 \\
\hline $\mathrm{H}$ & -4.814537 & -2.194594 & -3.076551 \\
\hline $\mathrm{H}$ & -3.155369 & 1.627419 & -4.148815 \\
\hline $\mathrm{H}$ & -4.454055 & -0.395854 & -4.762913 \\
\hline $\mathrm{C}$ & -2.616691 & -1.392353 & 1. 346120 \\
\hline $\mathrm{C}$ & -3.022996 & -3.859957 & 2.595692 \\
\hline $\mathrm{C}$ & -3.914780 & -1.832197 & 1.643560 \\
\hline $\mathrm{C}$ & -1.528934 & -2.196048 & 1.693317 \\
\hline $\mathrm{C}$ & -1.733079 & -3.424602 & 2.316700 \\
\hline $\mathrm{C}$ & -4.114805 & -3.060397 & 2.260353 \\
\hline $\mathrm{H}$ & -4.772206 & -1.204647 & 1.397165 \\
\hline $\mathrm{H}$ & -0.512225 & -1.851800 & 1.503858 \\
\hline $\mathrm{H}$ & -0.876294 & -4.037687 & 2.595776 \\
\hline $\mathrm{H}$ & -5.125916 & -3.393547 & 2.486192 \\
\hline $\mathrm{H}$ & -3.182711 & -4.819348 & 3.084288 \\
\hline $\mathrm{C}$ & -3.391947 & 1.374165 & 1.434423 \\
\hline $\mathrm{C}$ & -4.807450 & 3.270264 & 2.931434 \\
\hline $\mathrm{C}$ & -3.665853 & 1.151592 & 2.789099 \\
\hline $\mathrm{C}$ & -3.828822 & 2.563374 & 0.840474 \\
\hline $\mathrm{C}$ & -4.533875 & 3.501782 & 1.586852 \\
\hline $\mathrm{C}$ & -4.373640 & 2.091827 & 3.529746 \\
\hline $\mathrm{H}$ & -3.326671 & 0.236916 & 3.275028 \\
\hline $\mathrm{H}$ & -3.610454 & 2.768068 & -0.205310 \\
\hline $\mathrm{H}$ & -4.873375 & 4.420105 & 1.111237 \\
\hline $\mathrm{H}$ & -4.585811 & 1.900115 & 4.580108 \\
\hline $\mathrm{H}$ & -5.360067 & 4.006696 & 3.511584 \\
\hline $\mathrm{C}$ & 0.024812 & 2.054327 & -2.864119 \\
\hline $\mathrm{C}$ & -0.789698 & 4.291427 & -4.349922 \\
\hline $\mathrm{C}$ & -1.243587 & 2.622888 & -2.685462 \\
\hline $\mathrm{C}$ & 0.867189 & 2.621692 & -3.827123 \\
\hline $\mathrm{C}$ & 0.460553 & 3.723576 & -4.571562 \\
\hline $\mathrm{C}$ & -1.647035 & 3.739135 & -3.403829 \\
\hline $\mathrm{H}$ & 1.860337 & 2.203829 & -3.986801 \\
\hline $\mathrm{H}$ & 1.129997 & 4.146819 & -5.317068 \\
\hline $\mathrm{H}$ & -2.634620 & 4.156073 & -3.214044 \\
\hline $\mathrm{H}$ & -1.103836 & 5.164939 & -4.917354 \\
\hline $\mathrm{C}$ & 2.399082 & 0.669334 & -2.070091 \\
\hline $\mathrm{C}$ & 5.149313 & 0.696323 & -2.551577 \\
\hline $\mathrm{C}$ & 3.215005 & 1.629725 & -1.461467 \\
\hline $\mathrm{C}$ & 2.970884 & -0.272140 & -2.927981 \\
\hline $\mathrm{C}$ & 4.344831 & -0.260493 & -3.160375 \\
\hline $\mathrm{C}$ & 4.581603 & 1.647771 & -1.704946 \\
\hline $\mathrm{H}$ & 2.771992 & 2.377642 & -0.799282 \\
\hline $\mathrm{H}$ & 2.346743 & -1.022708 & -3.412310 \\
\hline $\mathrm{H}$ & 4.784890 & -1.003579 & -3.822616 \\
\hline $\mathrm{H}$ & 5.206789 & 2.401442 & -1.230078 \\
\hline $\mathrm{H}$ & 6.221884 & 0.703375 & -2.735887 \\
\hline $\mathrm{C}$ & 1.463413 & 1.178222 & 2.413351 \\
\hline $\mathrm{C}$ & 0.094316 & 1.213795 & 2.756189 \\
\hline $\mathrm{H}$ & -0.368173 & 0.267138 & 3.050072 \\
\hline $\mathrm{C}$ & 2.313281 & 2.426461 & 2.571322 \\
\hline $\mathrm{H}$ & 2.785674 & 2.354485 & 3.566442 \\
\hline
\end{tabular}

\begin{tabular}{rrrr} 
H & 3.145698 & 2.416857 & 1.854465 \\
$\mathrm{C}$ & 1.494395 & 3.701807 & 2.490383 \\
$\mathrm{H}$ & 1.094320 & 3.818769 & 1.466384 \\
$\mathrm{H}$ & 2.131563 & 4.574246 & 2.684122 \\
$\mathrm{C}$ & 0.350469 & 3.633517 & 3.492318 \\
$\mathrm{C}$ & -0.607436 & 2.488340 & 3.172163 \\
$\mathrm{H}$ & -1.251704 & 2.274555 & 4.036711 \\
$\mathrm{H}$ & -1.303768 & 2.806094 & 2.376795 \\
$\mathrm{O}$ & -2.102800 & 2.085701 & -1.749693 \\
$\mathrm{O}$ & 3.570246 & -0.279808 & 2.265276 \\
$\mathrm{~B}$ & 2.213796 & -0.196250 & 2.288320 \\
$\mathrm{O}$ & 1.580665 & -1.400944 & 2.139799 \\
$\mathrm{C}$ & 2.609182 & -2.421905 & 2.222755 \\
$\mathrm{C}$ & 3.895021 & -1.627321 & 1.833944 \\
$\mathrm{H}$ & 1.575671 & 1.341168 & 0.685198 \\
$\mathrm{C}$ & 4.119988 & -1.552154 & 0.333077 \\
$\mathrm{H}$ & 3.212219 & -1.231977 & -0.199156 \\
$\mathrm{H}$ & 4.442389 & -2.516681 & -0.079447 \\
$\mathrm{H}$ & 4.900981 & -0.810349 & 0.125291 \\
$\mathrm{C}$ & 5.157061 & -2.076700 & 2.536129 \\
$\mathrm{H}$ & 5.075261 & -1.985978 & 3.624173 \\
$\mathrm{H}$ & 6.002224 & -1.459837 & 2.208213 \\
$\mathrm{H}$ & 5.385499 & -3.122185 & 2.287300 \\
$\mathrm{C}$ & 2.250337 & -3.549438 & 1.277724 \\
$\mathrm{H}$ & 2.117379 & -3.199251 & 0.247331 \\
$\mathrm{H}$ & 1.313510 & -4.024133 & 1.600971 \\
$\mathrm{H}$ & 3.031980 & -4.321188 & 1.281155 \\
$\mathrm{C}$ & 2.609538 & -2.916300 & 3.659655 \\
$\mathrm{H}$ & 2.872496 & -2.114264 & 4.361205 \\
$\mathrm{H}$ & 3.311399 & -3.747068 & 3.803114 \\
$\mathrm{H}$ & 1.602213 & -3.269571 & 3.914533 \\
$\mathrm{H}$ & 0.775887 & 3.487571 & 4.498256 \\
$\mathrm{H}$ & -0.206398 & 4.578391 & 3.524819 \\
----------------------------------------- \\
$\mathrm{CF}$ & $2.09-2917.556118050$ & \\
\hline
\end{tabular}

SCF energy: -2917.556118050

M06-D3 (Gas Phase) /6-31G(d)+LANL2DZ (Pd) opt. 1 imaginary frequency: $518 \mathrm{i} \mathrm{cm-1}$ 


\begin{tabular}{|c|c|c|c|}
\hline \multicolumn{4}{|c|}{ Int-12 } \\
\hline $\mathrm{Pd}$ & -0.118862 & 0.850419 & 0.406422 \\
\hline $\mathrm{P}$ & 0.440179 & 0.551662 & -1.914288 \\
\hline $\mathrm{P}$ & -2.352024 & 0.176848 & 0.540555 \\
\hline $\mathrm{C}$ & -0.107189 & -1.035406 & -2.616224 \\
\hline $\mathrm{C}$ & -0.872592 & -3.553988 & -3.529265 \\
\hline $\mathrm{C}$ & -0.537227 & -1.197069 & -3.933445 \\
\hline $\mathrm{C}$ & -0.053816 & -2.141906 & -1.761680 \\
\hline $\mathrm{C}$ & -0.432663 & -3.398103 & -2.216359 \\
\hline $\mathrm{C}$ & -0.922740 & -2.456752 & -4.384492 \\
\hline $\mathrm{H}$ & -0.576725 & -0.338642 & -4.605182 \\
\hline $\mathrm{H}$ & 0.290963 & -2.006825 & -0.733771 \\
\hline $\mathrm{H}$ & -0.393759 & -4.253018 & -1.542111 \\
\hline $\mathrm{H}$ & -1.264990 & -2.580865 & -5.410356 \\
\hline $\mathrm{H}$ & -1.178161 & -4.535140 & -3.887888 \\
\hline $\mathrm{C}$ & -3.117016 & -0.053497 & -1.116565 \\
\hline $\mathrm{C}$ & -4.134991 & -0.465564 & -3.705104 \\
\hline $\mathrm{C}$ & -2.911304 & 0.884415 & -2.137878 \\
\hline $\mathrm{C}$ & -3.818101 & -1.220155 & -1.445261 \\
\hline $\mathrm{C}$ & -4.322106 & -1.429986 & -2.722348 \\
\hline $\mathrm{C}$ & -3.427285 & 0.692821 & -3.414984 \\
\hline $\mathrm{H}$ & -3.954320 & -1.994437 & -0.694158 \\
\hline $\mathrm{H}$ & -4.856619 & -2.350317 & -2.946215 \\
\hline $\mathrm{H}$ & -3.259875 & 1.447907 & -4.180524 \\
\hline $\mathrm{H}$ & -4.532559 & -0.614580 & -4.707104 \\
\hline $\mathrm{C}$ & -2.614312 & -1.422972 & 1.377296 \\
\hline $\mathrm{C}$ & -2.988626 & -3.942828 & 2.523265 \\
\hline $\mathrm{C}$ & -3.898655 & -1.853189 & 1.740053 \\
\hline $\mathrm{C}$ & -1.519925 & -2.258902 & 1.611944 \\
\hline $\mathrm{C}$ & -1.711447 & -3.517948 & 2.175884 \\
\hline $\mathrm{C}$ & -4.082518 & -3.105634 & 2.311623 \\
\hline $\mathrm{H}$ & -4.758866 & -1.204335 & 1.570837 \\
\hline $\mathrm{H}$ & -0.512173 & -1.914723 & 1.372286 \\
\hline $\mathrm{H}$ & -0.855555 & -4.170219 & 2.349082 \\
\hline $\mathrm{H}$ & -5.082677 & -3.431068 & 2.590863 \\
\hline $\mathrm{H}$ & -3.135016 & -4.925902 & 2.966722 \\
\hline $\mathrm{C}$ & -3.407050 & 1.354520 & 1.456276 \\
\hline $\mathrm{C}$ & -4.796762 & 3.274139 & 2.939288 \\
\hline $\mathrm{C}$ & -3.648439 & 1.157754 & 2.821266 \\
\hline $\mathrm{C}$ & -3.858406 & 2.528133 & 0.844382 \\
\hline $\mathrm{C}$ & -4.550490 & 3.479948 & 1.585273 \\
\hline $\mathrm{C}$ & -4.346558 & 2.110238 & 3.555202 \\
\hline $\mathrm{H}$ & -3.288615 & 0.256789 & 3.318275 \\
\hline $\mathrm{H}$ & -3.658940 & 2.709084 & -0.209932 \\
\hline $\mathrm{H}$ & -4.901470 & 4.388122 & 1.098834 \\
\hline $\mathrm{H}$ & -4.536956 & 1.941411 & 4.613485 \\
\hline $\mathrm{H}$ & -5.340844 & 4.020463 & 3.514825 \\
\hline $\mathrm{C}$ & -0.108517 & 1.880758 & -3.042930 \\
\hline C & -0.893688 & 4.120388 & -4.535972 \\
\hline $\mathrm{C}$ & -1.343658 & 2.505315 & -2.818850 \\
\hline $\mathrm{C}$ & 0.713152 & 2.391932 & -4.052745 \\
\hline $\mathrm{C}$ & 0.321739 & 3.496929 & -4.800793 \\
\hline $\mathrm{C}$ & -1.732356 & 3.622974 & -3.543193 \\
\hline $\mathrm{H}$ & 1.679912 & 1.925374 & -4.242487 \\
\hline $\mathrm{H}$ & 0.973689 & 3.878769 & -5.583349 \\
\hline $\mathrm{H}$ & -2.693688 & 4.083776 & -3.322815 \\
\hline $\mathrm{H}$ & -1.196599 & 4.995066 & -5.107768 \\
\hline $\mathrm{C}$ & 2.260053 & 0.532371 & -2.129317 \\
\hline $\mathrm{C}$ & 5.043310 & 0.597786 & -2.381876 \\
\hline $\mathrm{C}$ & 3.001991 & 1.576765 & -1.560512 \\
\hline $\mathrm{C}$ & 2.927604 & -0.480978 & -2.820554 \\
\hline $\mathrm{C}$ & 4.315950 & -0.446395 & -2.942068 \\
\hline $\mathrm{C}$ & 4.383755 & 1.613359 & -1.690543 \\
\hline $\mathrm{H}$ & 2.487279 & 2.383101 & -1.030537 \\
\hline $\mathrm{H}$ & 2.364226 & -1.298362 & -3.270385 \\
\hline $\mathrm{H}$ & 4.828325 & -1.240500 & -3.482136 \\
\hline $\mathrm{H}$ & 4.948404 & 2.433772 & -1.251382 \\
\hline $\mathrm{H}$ & 6.126947 & 0.621470 & -2.480160 \\
\hline $\mathrm{C}$ & 1.581163 & 1.232498 & 2.050087 \\
\hline $\mathrm{C}$ & 0.138763 & 1.239439 & 2.460422 \\
\hline $\mathrm{H}$ & -0.195357 & 0.374880 & 3.045717 \\
\hline $\mathrm{C}$ & 2.470512 & 2.350485 & 2.644138 \\
\hline $\mathrm{H}$ & 2.797343 & 2.006904 & 3.638170 \\
\hline
\end{tabular}

\begin{tabular}{|c|c|c|c|}
\hline $\mathrm{H}$ & 3.384163 & 2.437678 & 2.039403 \\
\hline C & 1.748153 & 3.677024 & 2.776477 \\
\hline $\mathrm{H}$ & 1.512153 & 4.078802 & 1.774242 \\
\hline $\mathrm{H}$ & 2.406718 & 4.410948 & 3.260078 \\
\hline C & 0.461015 & 3.497259 & 3.566282 \\
\hline C & -0.493257 & 2.568440 & 2.813431 \\
\hline $\mathrm{H}$ & -1.419775 & 2.408006 & 3.378390 \\
\hline $\mathrm{H}$ & -0.794703 & 3.092004 & 1.886380 \\
\hline O & -2.188642 & 2.022433 & -1.836433 \\
\hline 0 & 3.590261 & -0.364670 & 2.380363 \\
\hline B & 2.293434 & -0.174995 & 2.023814 \\
\hline O & 1.681769 & -1.305663 & 1.548156 \\
\hline $\mathrm{C}$ & 2.598282 & -2.405767 & 1.795197 \\
\hline C & 3.970610 & -1.672448 & 1.877256 \\
\hline $\mathrm{H}$ & 1.607251 & 1.532451 & 0.916522 \\
\hline C & 4.600546 & -1.435118 & 0.514947 \\
\hline $\mathrm{H}$ & 3.883007 & -0.996890 & -0.193181 \\
\hline $\mathrm{H}$ & 4.993459 & -2.363174 & 0.079933 \\
\hline $\mathrm{H}$ & 5.433104 & -0.729946 & 0.627662 \\
\hline C & 4.971209 & -2.294699 & 2.825307 \\
\hline $\mathrm{H}$ & 4.599771 & -2.323144 & 3.854838 \\
\hline $\mathrm{H}$ & 5.899587 & -1.711353 & 2.818136 \\
\hline $\mathrm{H}$ & 5.214206 & -3.319067 & 2.510993 \\
\hline C & 2.473220 & -3.402111 & 0.662619 \\
\hline $\mathrm{H}$ & 2.605198 & -2.925952 & -0.317087 \\
\hline $\mathrm{H}$ & 1.477809 & -3.868398 & 0.683627 \\
\hline $\mathrm{H}$ & 3.217420 & -4.203456 & 0.765916 \\
\hline $\mathrm{C}$ & 2.172440 & -3.033083 & 3.112398 \\
\hline $\mathrm{H}$ & 2.280107 & -2.329095 & 3.947512 \\
\hline $\mathrm{H}$ & 2.754915 & -3.934292 & 3.340419 \\
\hline $\mathrm{H}$ & 1.113148 & -3.315251 & 3.049457 \\
\hline $\mathrm{H}$ & 0.695520 & 3.074141 & 4.556772 \\
\hline $\mathrm{H}$ & -0.034266 & 4.460805 & 3.743210 \\
\hline
\end{tabular}

SCF energy: -2917.566005290

M06-D3 (Gas Phase) /6-31G(d)+LANL2DZ (Pd) opt. No imaginary frequency 


\begin{tabular}{|c|c|c|c|}
\hline \multicolumn{4}{|c|}{ Int-12prime } \\
\hline $\mathrm{Pd}$ & 1.294482 & -0.869485 & 0.000941 \\
\hline $\mathrm{P}$ & 1.469643 & -0.957032 & -2.330180 \\
\hline $\mathrm{P}$ & -1.018195 & -0.237777 & 0.423778 \\
\hline $\mathrm{C}$ & -0.086446 & -1.480717 & -3.115772 \\
\hline $\mathrm{C}$ & -2.484831 & -2.346185 & -4.238944 \\
\hline $\mathrm{C}$ & -0.525879 & -0.939827 & -4.325520 \\
\hline $\mathrm{C}$ & -0.843308 & -2.472308 & -2.485474 \\
\hline $\mathrm{C}$ & -2.040171 & -2.904046 & -3.042807 \\
\hline $\mathrm{C}$ & -1.725298 & -1.372847 & -4.881933 \\
\hline $\mathrm{H}$ & 0.061927 & -0.170675 & -4.827459 \\
\hline $\mathrm{H}$ & -0.488829 & -2.897542 & -1.545094 \\
\hline $\mathrm{H}$ & -2.629929 & -3.666042 & -2.535824 \\
\hline $\mathrm{H}$ & -2.072210 & -0.940518 & -5.818578 \\
\hline $\mathrm{H}$ & -3.427413 & -2.673279 & -4.673702 \\
\hline $\mathrm{C}$ & -1.822374 & 0.631246 & -0.971618 \\
\hline $\mathrm{C}$ & -2.909649 & 1.873071 & -3.237357 \\
\hline $\mathrm{C}$ & -1.042873 & 1.461774 & -1.788818 \\
\hline $\mathrm{C}$ & -3.154423 & 0.419071 & -1.337032 \\
\hline $\mathrm{C}$ & -3.697565 & 1.031475 & -2.460908 \\
\hline $\mathrm{C}$ & -1.576564 & 2.090292 & -2.906866 \\
\hline $\mathrm{H}$ & -3.770992 & -0.251513 & -0.740607 \\
\hline $\mathrm{H}$ & -4.736632 & 0.850500 & -2.726583 \\
\hline $\mathrm{H}$ & -0.950498 & 2.736530 & -3.518930 \\
\hline $\mathrm{H}$ & -3.328555 & 2.362470 & -4.114456 \\
\hline $\mathrm{C}$ & -2.287673 & -1.361925 & 1.093734 \\
\hline $\mathrm{C}$ & -4.180413 & -3.135852 & 2.120863 \\
\hline $\mathrm{C}$ & -3.437944 & -0.876299 & 1.728187 \\
\hline $\mathrm{C}$ & -2.086692 & -2.740880 & 0.999561 \\
\hline $\mathrm{C}$ & -3.032248 & -3.626528 & 1.508489 \\
\hline $\mathrm{C}$ & -4.381190 & -1.761141 & 2.234775 \\
\hline $\mathrm{H}$ & -3.588311 & 0.199636 & 1.829508 \\
\hline $\mathrm{H}$ & -1.172088 & -3.120802 & 0.541783 \\
\hline $\mathrm{H}$ & -2.866788 & -4.699748 & 1.435177 \\
\hline $\mathrm{H}$ & -5.273693 & -1.378971 & 2.726461 \\
\hline $\mathrm{H}$ & -4.919488 & -3.826530 & 2.522479 \\
\hline $\mathrm{C}$ & -0.842682 & 1.021641 & 1.746578 \\
\hline $\mathrm{C}$ & -0.379562 & 2.841715 & 3.816364 \\
\hline $\mathrm{C}$ & -0.487772 & 0.569132 & 3.024458 \\
\hline $\mathrm{C}$ & -0.966423 & 2.392140 & 1.517972 \\
\hline $\mathrm{C}$ & -0.730329 & 3.297068 & 2.550399 \\
\hline $\mathrm{C}$ & -0.264640 & 1.472704 & 4.054735 \\
\hline $\mathrm{H}$ & -0.399839 & -0.502756 & 3.216995 \\
\hline $\mathrm{H}$ & -1.252314 & 2.759569 & 0.533281 \\
\hline $\mathrm{H}$ & -0.829899 & 4.364651 & 2.363032 \\
\hline $\mathrm{H}$ & 0.000004 & 1.109677 & 5.046376 \\
\hline $\mathrm{H}$ & -0.199444 & 3.551811 & 4.621099 \\
\hline $\mathrm{C}$ & 1.840571 & 0.714684 & -2.975714 \\
\hline $\mathrm{C}$ & 2.594062 & 3.327993 & -3.671165 \\
\hline $\mathrm{C}$ & 1.227041 & 1.830515 & -2.384885 \\
\hline $\mathrm{C}$ & 2.810494 & 0.944820 & -3.957500 \\
\hline $\mathrm{C}$ & 3.179952 & 2.239146 & -4.308500 \\
\hline $\mathrm{C}$ & 1.611599 & 3.124052 & -2.707326 \\
\hline $\mathrm{H}$ & 3.296426 & 0.097345 & -4.438688 \\
\hline $\mathrm{H}$ & 3.937258 & 2.394409 & -5.073684 \\
\hline $\mathrm{H}$ & 1.129023 & 3.955137 & -2.195764 \\
\hline $\mathrm{H}$ & 2.896121 & 4.341332 & -3.927278 \\
\hline $\mathrm{C}$ & 2.747889 & -2.051122 & -3.043683 \\
\hline $\mathrm{C}$ & 4.716827 & -3.820862 & -3.951243 \\
\hline $\mathrm{C}$ & 4.105227 & -1.793425 & -2.801718 \\
\hline $\mathrm{C}$ & 2.391080 & -3.204406 & -3.748926 \\
\hline C & 3.373692 & -4.081964 & -4.199019 \\
\hline $\mathrm{C}$ & 5.080345 & -2.671219 & -3.253981 \\
\hline $\mathrm{H}$ & 4.425336 & -0.898875 & -2.271329 \\
\hline $\mathrm{H}$ & 1.343666 & -3.421639 & -3.953103 \\
\hline $\mathrm{H}$ & 3.083056 & -4.974118 & -4.750338 \\
\hline $\mathrm{H}$ & 6.126683 & -2.444430 & -3.055076 \\
\hline $\mathrm{H}$ & 5.480431 & -4.511100 & -4.304611 \\
\hline 0 & 0.272290 & 1.646918 & -1.406786 \\
\hline $\mathrm{C}$ & 3.937579 & 0.561420 & -0.024925 \\
\hline $\mathrm{C}$ & 3.005422 & -1.052800 & 1.721950 \\
\hline $\mathrm{C}$ & 3.223004 & 1.419976 & 2.301658 \\
\hline $\mathrm{C}$ & 3.561397 & -0.016075 & 2.698529 \\
\hline
\end{tabular}

\begin{tabular}{|c|c|c|c|}
\hline C & 3.263444 & 1.676467 & 0.781498 \\
\hline C & 3.363278 & -0.802954 & 0.294401 \\
\hline $\mathrm{H}$ & 2.220709 & 1.672509 & 2.679063 \\
\hline $\mathrm{H}$ & 3.771027 & 2.627812 & 0.573003 \\
\hline $\mathrm{H}$ & 3.829948 & -1.644688 & -0.22942 \\
\hline $\mathrm{H}$ & 3.786775 & 0.771358 & -1.099281 \\
\hline $\mathrm{H}$ & 3.223484 & -2.086078 & 2.019183 \\
\hline $\mathrm{H}$ & 3.923439 & 2.087901 & 2.818290 \\
\hline $\mathrm{H}$ & 2.233361 & 1.799888 & 0.408430 \\
\hline B & 5.505036 & 0.442947 & 0.139018 \\
\hline 0 & 6.244937 & -0.316458 & -0.729272 \\
\hline C & 7.520634 & -0.537458 & -0.077399 \\
\hline C & 7.629724 & 0.705326 & 0.856713 \\
\hline 0 & 6.236102 & 0.991710 & 1.148054 \\
\hline $\mathrm{C}$ & 8.190373 & 1.933309 & 0.158504 \\
\hline $\mathrm{H}$ & 7.687425 & 2.116915 & -0.799790 \\
\hline $\mathrm{H}$ & 9.267430 & 1.836161 & -0.026739 \\
\hline $\mathrm{H}$ & 8.031064 & 2.809163 & 0.798595 \\
\hline $\mathrm{C}$ & 8.605772 & -0.644160 & -1.12566 \\
\hline $\mathrm{H}$ & 8.458449 & -1.550726 & -1.72626 \\
\hline $\mathrm{H}$ & 9.594700 & -0.715378 & -0.652601 \\
\hline $\mathrm{H}$ & 8.604179 & 0.215872 & -1.803288 \\
\hline C & 7. 382237 & -1.850410 & 0.679582 \\
\hline $\mathrm{H}$ & 7.076588 & -2.634073 & -0.025671 \\
\hline $\mathrm{H}$ & 6.612821 & -1.784910 & 1. 462521 \\
\hline $\mathrm{H}$ & 8.325578 & -2.157059 & 1.148217 \\
\hline C & 8.356700 & 0.459664 & 2.160768 \\
\hline $\mathrm{H}$ & 7.865126 & -0.312351 & 2.763102 \\
\hline $\mathrm{H}$ & 8.382670 & 1.383177 & 2.751501 \\
\hline $\mathrm{H}$ & 9.393936 & 0.149764 & 1.973304 \\
\hline $\mathrm{H}$ & 1.850798 & -0.994443 & 1.866732 \\
\hline $\mathrm{H}$ & 3.195125 & -0.226630 & 3.712200 \\
\hline $\mathrm{H}$ & 4.653564 & -0.146218 & 2.725034 \\
\hline
\end{tabular}

SCF energy: -2917.558433010

M06-D3 (Gas Phase) /6-31G(d)+LANL2DZ (Pd) opt. No imaginary frequency 


\begin{tabular}{|c|c|c|c|}
\hline \multicolumn{4}{|l|}{ TS-10 } \\
\hline $\mathrm{Pd}$ & -0.714198 & -0.958045 & 1.006995 \\
\hline $\mathrm{P}$ & -0.349010 & -0.943026 & -1.411924 \\
\hline $\mathrm{P}$ & -2.944706 & -0.211357 & 1.193655 \\
\hline $\mathrm{C}$ & -1.781472 & -1.429277 & -2.430985 \\
\hline $\mathrm{C}$ & -3.912852 & -2.345753 & -3.975627 \\
\hline $\mathrm{C}$ & -1.929249 & -0.991962 & -3.749604 \\
\hline $\mathrm{C}$ & -2.700558 & -2.335106 & -1.897346 \\
\hline $\mathrm{C}$ & -3.763892 & -2.794162 & -2.666488 \\
\hline $\mathrm{C}$ & -2.995863 & -1.447155 & -4.516281 \\
\hline $\mathrm{H}$ & -1.211371 & -0.288982 & -4.174622 \\
\hline $\mathrm{H}$ & -2.578945 & -2.679106 & -0.868599 \\
\hline $\mathrm{H}$ & -4.478626 & -3.495113 & -2.238611 \\
\hline $\mathrm{H}$ & -3.111878 & -1.100112 & -5.541345 \\
\hline $\mathrm{H}$ & -4.746575 & -2.698879 & -4.579687 \\
\hline $\mathrm{C}$ & -3.610938 & 0.568745 & -0.315169 \\
\hline $\mathrm{C}$ & -4.446467 & 1.672133 & -2.747166 \\
\hline $\mathrm{C}$ & -2.771121 & 1.411679 & -1.054006 \\
\hline $\mathrm{C}$ & -4.874989 & 0.278173 & -0.833186 \\
\hline $\mathrm{C}$ & -5.292380 & 0.822491 & -2.042522 \\
\hline $\mathrm{C}$ & -3.180572 & 1.972880 & -2.256101 \\
\hline $\mathrm{H}$ & -5.532190 & -0.401606 & -0.292807 \\
\hline $\mathrm{H}$ & -6.277124 & 0.578733 & -2.434554 \\
\hline $\mathrm{H}$ & -2.507990 & 2.628940 & -2.805787 \\
\hline $\mathrm{H}$ & -4.768026 & 2.103233 & -3.693166 \\
\hline $\mathrm{C}$ & -4.223043 & -1.372019 & 1.763240 \\
\hline $\mathrm{C}$ & -6.149096 & -3.195411 & 2.617408 \\
\hline $\mathrm{C}$ & -5.451899 & -0.916365 & 2.255748 \\
\hline $\mathrm{C}$ & -3.960582 & -2.743197 & 1.721506 \\
\hline $\mathrm{C}$ & -4.924112 & -3.653893 & 2.143883 \\
\hline $\mathrm{C}$ & -6.411551 & -1.827808 & 2.677678 \\
\hline $\mathrm{H}$ & -5.651650 & 0.154827 & 2.313111 \\
\hline $\mathrm{H}$ & -2.986020 & -3.092794 & 1.375175 \\
\hline $\mathrm{H}$ & -4.713772 & -4.721160 & 2.112710 \\
\hline $\mathrm{H}$ & -7.365566 & -1.471560 & 3.061317 \\
\hline $\mathrm{H}$ & -6.901987 & -3.906267 & 2.952600 \\
\hline $\mathrm{C}$ & -2.935844 & 1.116771 & 2.455481 \\
\hline $\mathrm{C}$ & -2.739027 & 3.080166 & 4.432908 \\
\hline $\mathrm{C}$ & -2.616169 & 0.767474 & 3.772507 \\
\hline $\mathrm{C}$ & -3.179042 & 2.454167 & 2.143987 \\
\hline $\mathrm{C}$ & -3.074765 & 3.431668 & 3.131279 \\
\hline $\mathrm{C}$ & -2.520364 & 1.741566 & 4.756307 \\
\hline $\mathrm{H}$ & -2.442258 & -0.280344 & 4.028985 \\
\hline $\mathrm{H}$ & -3.450359 & 2.741122 & 1.129349 \\
\hline $\mathrm{H}$ & -3.260736 & 4.473559 & 2.877412 \\
\hline $\mathrm{H}$ & -2.274356 & 1.457405 & 5.778132 \\
\hline $\mathrm{H}$ & -2.654492 & 3.846718 & 5.200741 \\
\hline $\mathrm{C}$ & 0.143019 & 0.734685 & -1.969778 \\
\hline $\mathrm{C}$ & 1.124807 & 3.335654 & -2.382070 \\
\hline $\mathrm{C}$ & -0.465110 & 1.849500 & -1.370967 \\
\hline $\mathrm{C}$ & 1.225297 & 0.967784 & -2.825327 \\
\hline $\mathrm{C}$ & 1.709234 & 2.255369 & -3.035708 \\
\hline $\mathrm{C}$ & 0.024964 & 3.134374 & -1.554106 \\
\hline $\mathrm{H}$ & 1.713813 & 0.123896 & -3.311091 \\
\hline $\mathrm{H}$ & 2.554709 & 2.411198 & -3.702904 \\
\hline $\mathrm{H}$ & -0.465379 & 3.958485 & -1.039380 \\
\hline $\mathrm{H}$ & 1.516482 & 4.340623 & -2.524543 \\
\hline $\mathrm{C}$ & 0.984706 & -2.046665 & -2.015705 \\
\hline $\mathrm{C}$ & 3.007507 & -3.860514 & -2.700812 \\
\hline $\mathrm{C}$ & 2.318104 & -1.800664 & -1.658855 \\
\hline $\mathrm{C}$ & 0.681160 & -3.212805 & -2.724623 \\
\hline $\mathrm{C}$ & 1.689533 & -4.110446 & -3.065799 \\
\hline $\mathrm{C}$ & 3.319591 & -2.700484 & -1.996323 \\
\hline $\mathrm{H}$ & 2.599541 & -0.897273 & -1.120534 \\
\hline $\mathrm{H}$ & -0.346021 & -3.426690 & -3.016721 \\
\hline $\mathrm{H}$ & 1.438111 & -5.011610 & -3.621823 \\
\hline $\mathrm{H}$ & 4.343988 & -2.488732 & -1.692997 \\
\hline $\mathrm{H}$ & 3.790869 & -4.568423 & -2.964453 \\
\hline 0 & -1.530291 & 1.673741 & -0.512359 \\
\hline $\mathrm{C}$ & 1.902905 & 0.552244 & 1.136839 \\
\hline $\mathrm{C}$ & 0.953733 & -1.175507 & 2.725136 \\
\hline $\mathrm{C}$ & 1.666754 & 1.126036 & 3.549561 \\
\hline C & 0.989948 & -0.201841 & 3.891843 \\
\hline
\end{tabular}

\begin{tabular}{|c|c|c|c|}
\hline C & 1.343297 & 1.573126 & 2.131581 \\
\hline $\mathrm{C}$ & 1.469125 & -0.853847 & 1.462669 \\
\hline $\mathrm{H}$ & 1.368198 & 1.886883 & 4.282240 \\
\hline $\mathrm{H}$ & 1.780400 & 2.558634 & 1.925630 \\
\hline $\mathrm{H}$ & 1.862179 & -1.672868 & 0.855805 \\
\hline $\mathrm{H}$ & 1.531979 & 0.825431 & 0.130314 \\
\hline $\mathrm{H}$ & 0.917241 & -2.235694 & 2.982165 \\
\hline $\mathrm{H}$ & 2.761540 & 1.020057 & 3.640101 \\
\hline $\mathrm{H}$ & 0.249852 & 1.674198 & 2.008103 \\
\hline B & 3.460288 & 0.672454 & 0.929896 \\
\hline 0 & 4.259381 & -0.381790 & 0.579619 \\
\hline C & 5.615208 & 0.134489 & 0.529445 \\
\hline $\mathrm{C}$ & 5.373459 & 1.651555 & 0.278796 \\
\hline 0 & 4.107651 & 1.872699 & 0.948447 \\
\hline $\mathrm{C}$ & 5.138998 & 1.979896 & -1.189102 \\
\hline $\mathrm{H}$ & 4.395303 & 1.303717 & -1.637644 \\
\hline $\mathrm{H}$ & 6.064479 & 1.917481 & -1.775266 \\
\hline $\mathrm{H}$ & 4.749582 & 3.002805 & -1.264178 \\
\hline $\mathrm{C}$ & 6.371946 & -0.580343 & -0.568820 \\
\hline $\mathrm{H}$ & 6.495046 & -1.639203 & -0.307388 \\
\hline $\mathrm{H}$ & 7.374248 & -0.148492 & -0.694365 \\
\hline $\mathrm{H}$ & 5.847549 & -0.522701 & -1.530269 \\
\hline C & 6.242535 & -0.157041 & 1.882912 \\
\hline $\mathrm{H}$ & 6.177159 & -1.233583 & 2.081215 \\
\hline $\mathrm{H}$ & 5.717731 & 0.370014 & 2.690903 \\
\hline $\mathrm{H}$ & 7.300343 & 0.132951 & 1.909401 \\
\hline $\mathrm{C}$ & 6.416425 & 2.573505 & 0.869295 \\
\hline $\mathrm{H}$ & 6.482962 & 2.467731 & 1.957045 \\
\hline $\mathrm{H}$ & 6.159999 & 3.616243 & 0.646400 \\
\hline $\mathrm{H}$ & 7.404861 & 2.368314 & 0.435666 \\
\hline $\mathrm{H}$ & -0.833936 & -1.171636 & 2.599987 \\
\hline $\mathrm{H}$ & -0.019785 & -0.015744 & 4.280274 \\
\hline $\mathrm{H}$ & 1.531364 & -0.701865 & 4.707992 \\
\hline
\end{tabular}

SCF energy: -2917.539707090

M06-D3 (Gas Phase) /6-31G(d)+LANL2DZ (Pd) opt. 1 imaginary frequency: $555 \mathrm{i} \mathrm{cm-1}$ 


\begin{tabular}{|c|c|c|c|}
\hline \multicolumn{4}{|c|}{ Int-13 } \\
\hline $\mathrm{Pd}$ & 0.435842 & 0.007962 & 2.568801 \\
\hline $\mathrm{P}$ & 0.869712 & 0.104448 & 0.120840 \\
\hline $\mathrm{P}$ & -1.734645 & 0.823586 & 2.723887 \\
\hline $\mathrm{C}$ & -0.567181 & -0.386964 & -0.892814 \\
\hline $\mathrm{C}$ & -2.695046 & -1.324916 & -2.430921 \\
\hline $\mathrm{C}$ & -0.730661 & 0.057537 & -2.206921 \\
\hline $\mathrm{C}$ & -1.469172 & -1.310955 & -0.360832 \\
\hline $\mathrm{C}$ & -2.530447 & -1.780913 & -1.126192 \\
\hline $\mathrm{C}$ & -1.795637 & -0.408004 & -2.970095 \\
\hline $\mathrm{H}$ & -0.026375 & 0.774411 & -2.631442 \\
\hline $\mathrm{H}$ & -1.333637 & -1.662494 & 0.663910 \\
\hline $\mathrm{H}$ & -3.230895 & -2.496826 & -0.699315 \\
\hline $\mathrm{H}$ & -1.923575 & -0.054855 & -3.991669 \\
\hline $\mathrm{H}$ & -3.526840 & -1.686637 & -3.032551 \\
\hline $\mathrm{C}$ & -2.380743 & 1.591194 & 1.202307 \\
\hline $\mathrm{C}$ & -3.215555 & 2.724088 & -1.213543 \\
\hline $\mathrm{C}$ & -1.544024 & 2.451470 & 0.481389 \\
\hline $\mathrm{C}$ & -3.639934 & 1.295052 & 0.675173 \\
\hline $\mathrm{C}$ & -4.056924 & 1.855249 & -0.526792 \\
\hline $\mathrm{C}$ & -1.954029 & 3.027929 & -0.713367 \\
\hline $\mathrm{H}$ & -4.293696 & 0.602250 & 1.202698 \\
\hline $\mathrm{H}$ & -5.037631 & 1.608890 & -0.927065 \\
\hline $\mathrm{H}$ & -1.285019 & 3.698135 & -1.250023 \\
\hline $\mathrm{H}$ & -3.537475 & 3.167814 & -2.153554 \\
\hline $\mathrm{C}$ & -3.025258 & -0.324908 & 3. 284349 \\
\hline $\mathrm{C}$ & -4.998917 & -2.110003 & 4.105423 \\
\hline $\mathrm{C}$ & -4.249323 & 0.155375 & 3.764695 \\
\hline $\mathrm{C}$ & -2.791317 & -1.700685 & 3.235376 \\
\hline $\mathrm{C}$ & -3.779316 & -2.592088 & 3.641001 \\
\hline $\mathrm{C}$ & -5.232411 & -0.737712 & 4.171998 \\
\hline $\mathrm{H}$ & -4.427992 & 1.230124 & 3.824545 \\
\hline $\mathrm{H}$ & -1.820036 & -2.068796 & 2.900017 \\
\hline $\mathrm{H}$ & -3.591707 & -3.663376 & 3.605170 \\
\hline $\mathrm{H}$ & -6.181897 & -0.362925 & 4.548952 \\
\hline $\mathrm{H}$ & -5.770154 & -2.806539 & 4.428647 \\
\hline $\mathrm{C}$ & -1.716030 & 2.166326 & 3.967439 \\
\hline $\mathrm{C}$ & -1.584419 & 4.163099 & 5.915699 \\
\hline $\mathrm{C}$ & -1.369061 & 1.849183 & 5.285457 \\
\hline $\mathrm{C}$ & -2.018390 & 3.488617 & 3.640052 \\
\hline $\mathrm{C}$ & -1.946739 & 4.482606 & 4.612826 \\
\hline $\mathrm{C}$ & -1.305301 & 2.840063 & 6.254666 \\
\hline $\mathrm{H}$ & -1.146568 & 0.814590 & 5.555129 \\
\hline $\mathrm{H}$ & -2.312043 & 3.751422 & 2.625235 \\
\hline $\mathrm{H}$ & -2.178381 & 5.512094 & 4.346599 \\
\hline $\mathrm{H}$ & -1.034938 & 2.580623 & 7.276628 \\
\hline $\mathrm{H}$ & -1.524049 & 4.943220 & 6.672035 \\
\hline $\mathrm{C}$ & 1.352589 & 1.785109 & -0.440795 \\
\hline C & 2.336577 & 4.388410 & -0.837978 \\
\hline $\mathrm{C}$ & 0.755569 & 2.896082 & 0.174901 \\
\hline $\mathrm{C}$ & 2.420225 & 2.025481 & -1.312729 \\
\hline $\mathrm{C}$ & 2.904919 & 3.314093 & -1.515251 \\
\hline $\mathrm{C}$ & 1.246936 & 4.181453 & 0.002015 \\
\hline $\mathrm{H}$ & 2.897785 & 1.186852 & -1.817647 \\
\hline $\mathrm{H}$ & 3.739515 & 3.475229 & -2.194776 \\
\hline $\mathrm{H}$ & 0.765118 & 5.001099 & 0.531622 \\
\hline $\mathrm{H}$ & 2.730907 & 5.393314 & -0.973358 \\
\hline $\mathrm{C}$ & 2.198107 & -0.995167 & -0.508716 \\
\hline $\mathrm{C}$ & 4.208421 & -2.810273 & -1.233426 \\
\hline $\mathrm{C}$ & 3.543452 & -0.730634 & -0.214759 \\
\hline $\mathrm{C}$ & 1.877478 & -2.182020 & -1.175846 \\
\hline $\mathrm{C}$ & 2.878621 & -3.079351 & -1.537169 \\
\hline $\mathrm{C}$ & 4.538197 & -1.631229 & -0.570665 \\
\hline $\mathrm{H}$ & 3.840694 & 0.186388 & 0.290892 \\
\hline $\mathrm{H}$ & 0.841793 & -2.412109 & -1.421655 \\
\hline $\mathrm{H}$ & 2.612225 & -3.995457 & -2.060877 \\
\hline $\mathrm{H}$ & 5.571793 & -1.403823 & -0.314226 \\
\hline $\mathrm{H}$ & 4.986639 & -3.518034 & -1.512227 \\
\hline 0 & -0.309193 & 2.712524 & 1.033183 \\
\hline $\mathrm{C}$ & 3.152312 & 1.520633 & 2.622257 \\
\hline $\mathrm{C}$ & 2.292564 & -0.287315 & 4.146170 \\
\hline $\mathrm{C}$ & 2.906177 & 1.994778 & 5.058238 \\
\hline C & 2.253250 & 0.640816 & 5.337856 \\
\hline
\end{tabular}

\begin{tabular}{rrrr}
$\mathrm{C}$ & 2.582260 & 2.494496 & 3.657311 \\
$\mathrm{C}$ & 2.759423 & 0.097696 & 2.913448 \\
$\mathrm{H}$ & 2.591697 & 2.720725 & 5.819337 \\
$\mathrm{H}$ & 3.005724 & 3.493717 & 3.492414 \\
$\mathrm{H}$ & 3.066974 & -0.681642 & 2.213611 \\
$\mathrm{H}$ & 2.757675 & 1.812207 & 1.630726 \\
$\mathrm{H}$ & 2.175004 & -1.354129 & 4.342743 \\
$\mathrm{H}$ & 4.002276 & 1.902723 & 5.150470 \\
$\mathrm{H}$ & 1.487495 & 2.584551 & 3.534953 \\
$\mathrm{~B}$ & 4.703189 & 1.682050 & 2.400283 \\
$\mathrm{O}$ & 5.519301 & 0.659928 & 1.996859 \\
$\mathrm{C}$ & 6.864756 & 1.202681 & 1.964541 \\
$\mathrm{C}$ & 6.593780 & 2.724131 & 1.779695 \\
$\mathrm{O}$ & 5.328575 & 2.892815 & 2.464827 \\
$\mathrm{C}$ & 6.344204 & 3.109305 & 0.328179 \\
$\mathrm{H}$ & 5.613934 & 2.435526 & -0.145065 \\
$\mathrm{H}$ & 7.267736 & 3.093370 & -0.264122 \\
$\mathrm{H}$ & 5.930742 & 4.125121 & 0.298477 \\
$\mathrm{C}$ & 7.629890 & 0.548605 & 0.834708 \\
$\mathrm{H}$ & 7.770138 & -0.518498 & 1.049687 \\
$\mathrm{H}$ & 8.624905 & 1.001270 & 0.725651 \\
$\mathrm{H}$ & 7.101783 & 0.639525 & -0.121963 \\
$\mathrm{C}$ & 7.502643 & 0.865689 & 3.302577 \\
$\mathrm{H}$ & 7.456208 & -0.219149 & 3.455742 \\
$\mathrm{H}$ & 6.971798 & 1.349043 & 4.133598 \\
$\mathrm{H}$ & 8.555423 & 1.172633 & 3.337834 \\
$\mathrm{C}$ & 7.623163 & 3.640238 & 2.402441 \\
$\mathrm{H}$ & 7.695134 & 3.494239 & 3.485133 \\
$\mathrm{H}$ & 7.348349 & 4.686329 & 2.220830 \\
$\mathrm{H}$ & 8.613482 & 3.468961 & 1.958479 \\
$\mathrm{H}$ & -0.046829 & -0.280504 & 4.058123 \\
$\mathrm{H}$ & 1.222683 & 0.784629 & 5.688999 \\
$\mathrm{H}$ & 2.774446 & 0.134308 & 6.163991 \\
---------------------------------------- \\
$\mathrm{CF}$ & & .502917 .542366670 & \\
\hline
\end{tabular}

SCF energy: -2917.542366670

M06-D3 (Gas Phase) /6-31G(d)+LANL2DZ (Pd) opt. No imaginary frequency 


\begin{tabular}{|c|c|c|c|}
\hline \multicolumn{4}{|l|}{ TS-11 } \\
\hline $\mathrm{Pd}$ & -0.776767 & -0.323852 & 0.683305 \\
\hline $\mathrm{P}$ & -0.270848 & -0.452845 & -1.841878 \\
\hline $\mathrm{P}$ & -2.893202 & 0.680725 & 0.766058 \\
\hline $\mathrm{C}$ & -1.707998 & -0.892812 & -2.884050 \\
\hline $\mathrm{C}$ & -3.930191 & -1.662294 & -4.392992 \\
\hline $\mathrm{C}$ & -1.906170 & -0.369373 & -4.162836 \\
\hline $\mathrm{C}$ & -2.626623 & -1.816009 & -2.374807 \\
\hline $\mathrm{C}$ & -3.729803 & -2.204942 & -3.126482 \\
\hline $\mathrm{C}$ & -3.017607 & -0.747393 & -4.909389 \\
\hline $\mathrm{H}$ & -1.192794 & 0.345872 & -4.573407 \\
\hline $\mathrm{H}$ & -2.468662 & -2.230606 & -1.375249 \\
\hline $\mathrm{H}$ & -4.437051 & -2.928370 & -2.721815 \\
\hline $\mathrm{H}$ & -3.170131 & -0.326087 & -5.901438 \\
\hline $\mathrm{H}$ & -4.798188 & -1.956359 & -4.979965 \\
\hline $\mathrm{C}$ & -3.418441 & 1.456589 & -0.812493 \\
\hline $\mathrm{C}$ & -4.126194 & 2.590898 & -3.279341 \\
\hline $\mathrm{C}$ & -2.474568 & 2.133465 & -1.597749 \\
\hline $\mathrm{C}$ & -4.731463 & 1.387126 & -1.288491 \\
\hline $\mathrm{C}$ & -5.085871 & 1.939048 & -2.513789 \\
\hline $\mathrm{C}$ & -2.819567 & 2.700080 & -2.819562 \\
\hline $\mathrm{H}$ & -5.490780 & 0.884109 & -0.693319 \\
\hline $\mathrm{H}$ & -6.112295 & 1.861887 & -2.864974 \\
\hline $\mathrm{H}$ & -2.064154 & 3.219514 & -3.406238 \\
\hline $\mathrm{H}$ & -4.393259 & 3.027888 & -4.239611 \\
\hline $\mathrm{C}$ & -4.388366 & -0.236064 & 1.300721 \\
\hline $\mathrm{C}$ & -6.568577 & -1.799207 & 2.100912 \\
\hline $\mathrm{C}$ & -5.041001 & 0.027652 & 2.506883 \\
\hline $\mathrm{C}$ & -4.844444 & -1.288835 & 0.496988 \\
\hline $\mathrm{C}$ & -5.932328 & -2.057469 & 0.887738 \\
\hline $\mathrm{C}$ & -6.121129 & -0.757992 & 2.906585 \\
\hline $\mathrm{H}$ & -4.713073 & 0.852924 & 3.137758 \\
\hline $\mathrm{H}$ & -4.350861 & -1.497134 & -0.453575 \\
\hline $\mathrm{H}$ & -6.283143 & -2.864430 & 0.246366 \\
\hline $\mathrm{H}$ & -6.621521 & -0.542859 & 3.848985 \\
\hline $\mathrm{H}$ & -7.416173 & -2.406033 & 2.413319 \\
\hline $\mathrm{C}$ & -2.765825 & 2.092062 & 1.924659 \\
\hline $\mathrm{C}$ & -2.412406 & 4.199229 & 3.728694 \\
\hline $\mathrm{C}$ & -2.278287 & 1.852314 & 3.213577 \\
\hline $\mathrm{C}$ & -3.088442 & 3.398816 & 1.553743 \\
\hline $\mathrm{C}$ & -2.905150 & 4.446434 & 2.452007 \\
\hline $\mathrm{C}$ & -2.108354 & 2.895461 & 4.114485 \\
\hline $\mathrm{H}$ & -2.019416 & 0.831210 & 3.502534 \\
\hline $\mathrm{H}$ & -3.480177 & 3.604506 & 0.558433 \\
\hline $\mathrm{H}$ & -3.151010 & 5.462678 & 2.149718 \\
\hline $\mathrm{H}$ & -1.725553 & 2.692914 & 5.113790 \\
\hline $\mathrm{H}$ & -2.265743 & 5.021957 & 4.425785 \\
\hline $\mathrm{C}$ & 0.353825 & 1.132095 & -2.523419 \\
\hline $\mathrm{C}$ & 1.557208 & 3.617997 & -3.040863 \\
\hline $\mathrm{C}$ & -0.144943 & 2.318131 & -1.963694 \\
\hline $\mathrm{C}$ & 1.441642 & 1.234577 & -3.396622 \\
\hline $\mathrm{C}$ & 2.035201 & 2.465817 & -3.658537 \\
\hline $\mathrm{C}$ & 0.452568 & 3.547718 & -2.197583 \\
\hline $\mathrm{H}$ & 1.848360 & 0.331928 & -3.851711 \\
\hline $\mathrm{H}$ & 2.884109 & 2.522079 & -4.337497 \\
\hline $\mathrm{H}$ & 0.046160 & 4.430216 & -1.706704 \\
\hline $\mathrm{H}$ & 2.037442 & 4.577169 & -3.223134 \\
\hline $\mathrm{C}$ & 0.993496 & -1.694756 & -2.324845 \\
\hline $\mathrm{C}$ & 2.897808 & -3.719932 & -2.711223 \\
\hline $\mathrm{C}$ & 2.335318 & -1.496636 & -1.968091 \\
\hline $\mathrm{C}$ & 0.623052 & -2.922871 & -2.882309 \\
\hline $\mathrm{C}$ & 1.571060 & -3.925148 & -3.076877 \\
\hline $\mathrm{C}$ & 3.276728 & -2.499856 & -2.156351 \\
\hline $\mathrm{H}$ & 2.669492 & -0.552353 & -1.540524 \\
\hline $\mathrm{H}$ & -0.410765 & -3.101852 & -3.175703 \\
\hline $\mathrm{H}$ & 1.266849 & -4.870903 & -3.522100 \\
\hline $\mathrm{H}$ & 4.307666 & -2.318766 & -1.855476 \\
\hline $\mathrm{H}$ & 3.634325 & -4.507188 & -2.860892 \\
\hline 0 & -1.205270 & 2.255957 & -1.081694 \\
\hline $\mathrm{C}$ & 2.037192 & 1.170557 & 0.561526 \\
\hline $\mathrm{C}$ & 1.149251 & -0.334247 & 2.358806 \\
\hline $\mathrm{C}$ & 1.817998 & 2.045687 & 2.891260 \\
\hline $\mathrm{C}$ & 1.122604 & 0.774454 & 3.376965 \\
\hline
\end{tabular}

\begin{tabular}{|c|c|c|c|}
\hline C & 1.511011 & 2.319840 & 1.425631 \\
\hline C & 1.599852 & -0.170380 & 1.081224 \\
\hline $\mathrm{H}$ & 1.521780 & 2.895442 & 3.521151 \\
\hline $\mathrm{H}$ & 1.970368 & 3.263310 & 1.101680 \\
\hline $\mathrm{H}$ & 1.860806 & -1.062209 & 0.506005 \\
\hline $\mathrm{H}$ & 1.647629 & 1.312710 & -0.462152 \\
\hline $\mathrm{H}$ & 1.022608 & -1.353271 & 2.725994 \\
\hline $\mathrm{H}$ & 2.910343 & 1.935797 & 3.006593 \\
\hline $\mathrm{H}$ & 0.419958 & 2.427226 & 1.288736 \\
\hline B & 3.590145 & 1.223972 & 0.316220 \\
\hline O & 4.351953 & 0.110563 & 0.072803 \\
\hline C & 5.723666 & 0.566766 & -0.023652 \\
\hline $\mathrm{C}$ & 5.540676 & 2.057771 & -0.430914 \\
\hline O & 4.283613 & 2.394759 & 0.202929 \\
\hline C & 5.326545 & 2.239095 & -1.926778 \\
\hline $\mathrm{H}$ & 4.559782 & 1.547195 & -2.306623 \\
\hline $\mathrm{H}$ & 6.252014 & 2.082161 & -2.495156 \\
\hline $\mathrm{H}$ & 4.975221 & 3.262012 & -2.111709 \\
\hline $\mathrm{C}$ & 6.453406 & -0.287301 & -1.037777 \\
\hline $\mathrm{H}$ & 6.537130 & -1.316311 & -0.664921 \\
\hline $\mathrm{H}$ & 7.470848 & 0.091075 & -1.206980 \\
\hline $\mathrm{H}$ & 5.928041 & -0.313126 & -1.999828 \\
\hline $\mathrm{C}$ & 6.338561 & 0.393973 & 1.356060 \\
\hline $\mathrm{H}$ & 6.229798 & -0.652735 & 1.664658 \\
\hline $\mathrm{H}$ & 5.832275 & 1.021469 & 2.101676 \\
\hline $\mathrm{H}$ & 7.406897 & 0.644325 & 1.358364 \\
\hline $\mathrm{C}$ & 6.616582 & 2.996315 & 0.068112 \\
\hline $\mathrm{H}$ & 6.674915 & 3.002988 & 1.161506 \\
\hline $\mathrm{H}$ & 6.400972 & 4.018965 & -0.264420 \\
\hline $\mathrm{H}$ & 7.598109 & 2.709811 & -0.334170 \\
\hline $\mathrm{N}$ & -1.527864 & -2.533235 & 2.870264 \\
\hline C & -2.793812 & -3.134614 & 2.438742 \\
\hline $\mathrm{H}$ & -3.614847 & -2.446845 & 2.667399 \\
\hline $\mathrm{H}$ & -2.962710 & -4.091680 & 2.957498 \\
\hline $\mathrm{H}$ & -2.778901 & -3.313794 & 1.359228 \\
\hline C & -1.607780 & -2.101214 & 4.259538 \\
\hline $\mathrm{H}$ & -0.675396 & -1.605232 & 4.554361 \\
\hline $\mathrm{H}$ & -1.806346 & -2.938076 & 4.948446 \\
\hline $\mathrm{H}$ & -2.434689 & -1.384353 & 4.347218 \\
\hline $\mathrm{H}$ & -1.319367 & -1.111776 & 2.040336 \\
\hline C & -0.385883 & -3.332256 & 2.515074 \\
\hline C & 1.907426 & -4.718178 & 1.717608 \\
\hline C & -0.081636 & -3.479431 & 1.156960 \\
\hline $\mathrm{C}$ & 0.452337 & -3.904705 & 3.469255 \\
\hline $\mathrm{C}$ & 1.596800 & -4.591625 & 3.065115 \\
\hline C & 1.058456 & -4.162821 & 0.761169 \\
\hline $\mathrm{H}$ & -0.721292 & -3.008557 & 0.406419 \\
\hline $\mathrm{H}$ & 0.232293 & -3.813719 & 4.530658 \\
\hline $\mathrm{H}$ & 2.248610 & -5.027609 & 3.819768 \\
\hline $\mathrm{H}$ & 1.291575 & -4.244336 & -0.300977 \\
\hline $\mathrm{H}$ & 2.807602 & -5.245555 & 1.409338 \\
\hline $\mathrm{H}$ & 0.088480 & 1.005961 & 3.668135 \\
\hline $\mathrm{H}$ & 1.607731 & 0.403911 & 4.293740 \\
\hline
\end{tabular}

SCF energy: -3283.481861090

M06-D3 (Gas Phase) /6-31G(d)+LANL2DZ (Pd) opt. 1 imaginary frequency: $\quad 533 i \mathrm{~cm}-1$ 


\begin{tabular}{|c|c|c|c|}
\hline \multicolumn{4}{|c|}{ Int-14 } \\
\hline $\mathrm{Pd}$ & 1.134721 & 0.148653 & 0.689225 \\
\hline $\mathrm{P}$ & 1.347943 & 0.657725 & -1.636469 \\
\hline $\mathrm{P}$ & -1.229622 & 0.010208 & 1.154148 \\
\hline $\mathrm{C}$ & 0.648087 & -0.678240 & -2.694325 \\
\hline $\mathrm{C}$ & -0.443079 & -2.768707 & -4.194558 \\
\hline $\mathrm{C}$ & 0.433152 & -0.526361 & -4.067894 \\
\hline $\mathrm{C}$ & 0.318501 & -1.890756 & -2.085026 \\
\hline $\mathrm{C}$ & -0.224114 & -2.933007 & -2.831137 \\
\hline $\mathrm{C}$ & -0.110876 & -1.565103 & -4.813388 \\
\hline $\mathrm{H}$ & 0.684850 & 0.417641 & -4.554099 \\
\hline $\mathrm{H}$ & 0.480790 & -1.997232 & -1.008579 \\
\hline $\mathrm{H}$ & -0.487530 & -3.869538 & -2.341786 \\
\hline $\mathrm{H}$ & -0.279800 & -1.435586 & -5.881622 \\
\hline $\mathrm{H}$ & -0.876256 & -3.579480 & -4.778730 \\
\hline $\mathrm{C}$ & -2.281707 & -0.158492 & -0.348638 \\
\hline $\mathrm{C}$ & -3.416974 & -0.539895 & -2.886288 \\
\hline $\mathrm{C}$ & -2.088156 & 0.769446 & -1.382104 \\
\hline $\mathrm{C}$ & -3.090304 & -1.265400 & -0.614172 \\
\hline $\mathrm{C}$ & -3.655478 & -1.459151 & -1.871834 \\
\hline $\mathrm{C}$ & -2.639647 & 0.588693 & -2.643727 \\
\hline $\mathrm{H}$ & -3.261627 & -2.002287 & 0.170045 \\
\hline $\mathrm{H}$ & -4.272442 & -2.336114 & -2.059147 \\
\hline $\mathrm{H}$ & -2.447027 & 1.315522 & -3.431223 \\
\hline $\mathrm{H}$ & -3.837374 & -0.694476 & -3.878783 \\
\hline $\mathrm{C}$ & -1.945458 & -1.327768 & 2.198559 \\
\hline $\mathrm{C}$ & -3.000086 & -3.492037 & 3.628069 \\
\hline $\mathrm{C}$ & -3.069841 & -1.158486 & 3.013829 \\
\hline $\mathrm{C}$ & -1.354417 & -2.590694 & 2.120062 \\
\hline $\mathrm{C}$ & -1.876671 & -3.668574 & 2.826992 \\
\hline $\mathrm{C}$ & -3.592926 & -2.234126 & 3.723698 \\
\hline $\mathrm{H}$ & -3.541456 & -0.178892 & 3.091342 \\
\hline $\mathrm{H}$ & -0.468650 & -2.726009 & 1.496896 \\
\hline $\mathrm{H}$ & -1.387332 & -4.639422 & 2.770873 \\
\hline $\mathrm{H}$ & -4.469872 & -2.090471 & 4.353740 \\
\hline $\mathrm{H}$ & -3.411092 & -4.332894 & 4.185953 \\
\hline $\mathrm{C}$ & -1.887522 & 1.511619 & 1.980896 \\
\hline $\mathrm{C}$ & -2.790529 & 3.769595 & 3.360745 \\
\hline $\mathrm{C}$ & -1.089182 & 2.111274 & 2.958204 \\
\hline $\mathrm{C}$ & -3.141197 & 2.058704 & 1.697488 \\
\hline $\mathrm{C}$ & -3.589090 & 3.183519 & 2.382248 \\
\hline $\mathrm{C}$ & -1.540292 & 3.230782 & 3.649702 \\
\hline $\mathrm{H}$ & -0.099488 & 1.697595 & 3.161364 \\
\hline $\mathrm{H}$ & -3.769535 & 1.598290 & 0.933446 \\
\hline $\mathrm{H}$ & -4.566797 & 3.604531 & 2.151143 \\
\hline $\mathrm{H}$ & -0.908819 & 3.689716 & 4.409117 \\
\hline $\mathrm{H}$ & -3.141810 & 4.650999 & 3.895400 \\
\hline $\mathrm{C}$ & 0.606199 & 2.156027 & -2.431953 \\
\hline $\mathrm{C}$ & -0.600633 & 4.475143 & -3.486444 \\
\hline $\mathrm{C}$ & -0.669275 & 2.569442 & -2.025639 \\
\hline $\mathrm{C}$ & 1.252305 & 2.934926 & -3.398537 \\
\hline $\mathrm{C}$ & 0.658996 & 4.078610 & -3.923902 \\
\hline $\mathrm{C}$ & -1.267945 & 3.716105 & -2.532427 \\
\hline $\mathrm{H}$ & 2.245229 & 2.642394 & -3.739359 \\
\hline $\mathrm{H}$ & 1.187418 & 4.663234 & -4.675006 \\
\hline $\mathrm{H}$ & -2.252871 & 3.994068 & -2.160698 \\
\hline $\mathrm{H}$ & -1.065531 & 5.374417 & -3.886595 \\
\hline $\mathrm{C}$ & 3.085742 & 0.762795 & -2.223121 \\
\hline $\mathrm{C}$ & 5.823942 & 0.883318 & -2.809399 \\
\hline $\mathrm{C}$ & 3.867678 & 1.835361 & -1.772258 \\
\hline $\mathrm{C}$ & 3.699924 & -0.255983 & -2.954162 \\
\hline $\mathrm{C}$ & 5.062873 & -0.197519 & -3.238636 \\
\hline $\mathrm{C}$ & 5.220242 & 1.904671 & -2.076778 \\
\hline $\mathrm{H}$ & 3.406575 & 2.618056 & -1.165768 \\
\hline $\mathrm{H}$ & 3.113069 & -1.107155 & -3.298818 \\
\hline $\mathrm{H}$ & 5.528747 & -1.003312 & -3.804208 \\
\hline $\mathrm{H}$ & 5.811159 & 2.749851 & -1.726764 \\
\hline $\mathrm{H}$ & 6.887929 & 0.928653 & -3.036086 \\
\hline 0 & -1.335748 & 1.867517 & -1.049233 \\
\hline C & 2.316992 & -2.005227 & 2.621436 \\
\hline $\mathrm{C}$ & 3.186344 & 0.181289 & 1.637188 \\
\hline $\mathrm{C}$ & 4.063059 & -2.027254 & 0.813245 \\
\hline $\mathrm{C}$ & 4.292532 & -0.526027 & 0.898922 \\
\hline
\end{tabular}

\begin{tabular}{|c|c|c|c|}
\hline C & 3.670090 & -2.575528 & 2.180386 \\
\hline $\mathrm{C}$ & 2.302598 & -0.481959 & 2.480100 \\
\hline $\mathrm{H}$ & 3.252973 & -2.234933 & 0.090133 \\
\hline $\mathrm{H}$ & 4.443413 & -2.297639 & 2.917926 \\
\hline $\mathrm{H}$ & 1.837951 & 0.087587 & 3.290342 \\
\hline $\mathrm{H}$ & 1.551143 & -2.448322 & 1.962117 \\
\hline $\mathrm{H}$ & 3.329813 & 1.255541 & 1.789540 \\
\hline $\mathrm{H}$ & 4.966036 & -2.525140 & 0.431086 \\
\hline $\mathrm{H}$ & 3.626470 & -3.674989 & 2.164951 \\
\hline B & 1.916414 & -2.353372 & 4.092265 \\
\hline 0 & 0.820934 & -3.106067 & 4.432180 \\
\hline C & 0.549321 & -2.821449 & 5.818131 \\
\hline C & 1.960158 & -2.433442 & 6.351440 \\
\hline O & 2.573303 & -1.848423 & 5.186568 \\
\hline C & 2.802917 & -3.639533 & 6.740062 \\
\hline $\mathrm{H}$ & 2.796089 & -4.401721 & 5.950089 \\
\hline $\mathrm{H}$ & 2.450424 & -4.099392 & 7.672769 \\
\hline $\mathrm{H}$ & 3.840066 & -3.313678 & 6.886076 \\
\hline C & -0.071760 & -4.042820 & 6.462242 \\
\hline $\mathrm{H}$ & -1.050894 & -4.237766 & 6.005401 \\
\hline $\mathrm{H}$ & -0.224742 & -3.881370 & 7.538775 \\
\hline $\mathrm{H}$ & 0.549251 & -4.934872 & 6.326092 \\
\hline $\mathrm{C}$ & -0.437141 & -1.660553 & 5.838032 \\
\hline $\mathrm{H}$ & -1.337822 & -1.949125 & 5.281232 \\
\hline $\mathrm{H}$ & -0.016884 & -0.768874 & 5.349668 \\
\hline $\mathrm{H}$ & -0.733380 & -1.392933 & 6.860857 \\
\hline C & 1.961617 & -1.412811 & 7.470259 \\
\hline $\mathrm{H}$ & 1.504040 & -0.468996 & 7.155153 \\
\hline $\mathrm{H}$ & 2.993429 & -1.201234 & 7.777617 \\
\hline $\mathrm{H}$ & 1.416858 & -1.793334 & 8.346043 \\
\hline $\mathrm{H}$ & 4.442721 & -0.095226 & -0.100546 \\
\hline $\mathrm{H}$ & 5.236976 & -0.345445 & 1.446261 \\
\hline
\end{tabular}

SCF energy: -2917.143067120

M06-D3 (Gas Phase)/6-31G(d) +LANL2DZ (Pd) opt. No imaginary frequency 


\begin{tabular}{|c|c|c|c|}
\hline \multicolumn{4}{|c|}{ Product_12 } \\
\hline C & -0.084768 & -0.187723 & 0.975012 \\
\hline C & 1.109996 & 1.771263 & 1.995412 \\
\hline $\mathrm{C}$ & -0.887117 & 0.807853 & 3.147974 \\
\hline $\mathrm{C}$ & 0.026806 & 2.019812 & 3.003701 \\
\hline $\mathrm{C}$ & -1.297270 & 0.290886 & 1.775696 \\
\hline C & 1.079402 & 0.763838 & 1.119896 \\
\hline $\mathrm{H}$ & -0.349452 & 0.011890 & 3.689141 \\
\hline $\mathrm{H}$ & -1.796618 & 1.101608 & 1.220312 \\
\hline $\mathrm{H}$ & 1.921994 & 0.613276 & 0.439926 \\
\hline $\mathrm{H}$ & 0.244189 & -1.178758 & 1.343626 \\
\hline $\mathrm{H}$ & 1.970970 & 2. 442412 & 2.006053 \\
\hline $\mathrm{H}$ & -1.768767 & 1.062978 & 3.752511 \\
\hline $\mathrm{H}$ & -2.035449 & -0.519274 & 1.861749 \\
\hline B & -0.370146 & -0.336662 & -0.560640 \\
\hline 0 & 0.519376 & -0.928541 & -1.422217 \\
\hline $\mathrm{C}$ & 0.121169 & -0.537719 & -2.751018 \\
\hline C & -1.401199 & -0.261138 & -2.570472 \\
\hline O & -1.471943 & 0.170774 & -1.196022 \\
\hline $\mathrm{C}$ & -2.254063 & -1.516451 & -2.681485 \\
\hline $\mathrm{H}$ & -1.851366 & -2.328946 & -2.062912 \\
\hline $\mathrm{H}$ & -2.325844 & -1.869636 & -3.718420 \\
\hline $\mathrm{H}$ & -3.265618 & -1.288734 & -2.323864 \\
\hline $\mathrm{C}$ & 0.459413 & -1.650671 & -3.720156 \\
\hline $\mathrm{H}$ & 1.547957 & -1.776153 & -3.773993 \\
\hline $\mathrm{H}$ & 0.095167 & -1.411514 & -4.729321 \\
\hline $\mathrm{H}$ & 0.025690 & -2.606823 & -3.408577 \\
\hline C & 0.918456 & 0.714729 & -3.089751 \\
\hline $\mathrm{H}$ & 1.987139 & 0.489937 & -2.985302 \\
\hline $\mathrm{H}$ & 0.679876 & 1.537719 & -2.402168 \\
\hline $\mathrm{H}$ & 0.735223 & 1.053053 & -4.117892 \\
\hline $\mathrm{C}$ & -1.962355 & 0.830188 & -3.457938 \\
\hline $\mathrm{H}$ & -1.471361 & 1.792361 & -3.277702 \\
\hline $\mathrm{H}$ & -3.033689 & 0.955948 & -3.257818 \\
\hline $\mathrm{H}$ & -1.843755 & 0.567734 & -4.518752 \\
\hline $\mathrm{H}$ & 0.475390 & 2.281386 & 3.974406 \\
\hline $\mathrm{H}$ & -0.565435 & 2.903699 & 2.707503 \\
\hline
\end{tabular}

SCF energy: -644.868521087

M06-D3 (Gas Phase) /6-31G(d) +LANL2DZ (Pd) opt. No imaginary frequency

\begin{tabular}{|c|c|c|c|}
\hline \multicolumn{4}{|c|}{ Product_13 } \\
\hline $\mathrm{H}$ & 0.439755 & 4.349610 & 3.642103 \\
\hline $\mathrm{H}$ & 2.015868 & 2.663690 & 2.524462 \\
\hline $\mathrm{H}$ & 1.931779 & 2.530417 & 4.263425 \\
\hline $\mathrm{H}$ & 4.418505 & 0.207858 & 0.285245 \\
\hline $\mathrm{C}$ & 1.333122 & 2.396426 & 3.345028 \\
\hline $\mathrm{C}$ & 0.129587 & 3.330573 & 3.371822 \\
\hline $\mathrm{H}$ & 5.952575 & -0.180382 & 2.141900 \\
\hline $\mathrm{H}$ & -0.307689 & 3.391151 & 2.361138 \\
\hline O & 3.321692 & 0.217188 & 2.613398 \\
\hline $\mathrm{C}$ & 3.925288 & -0.742763 & 0.522234 \\
\hline $\mathrm{H}$ & 2.897229 & -0.694333 & 0.140495 \\
\hline $\mathrm{C}$ & -0.926663 & 2.808046 & 4.337047 \\
\hline $\mathrm{H}$ & 4.455802 & -1.547544 & -0.003297 \\
\hline $\mathrm{C}$ & 5.376379 & -1.033066 & 2.522291 \\
\hline $\mathrm{C}$ & 0.936300 & 0.944998 & 3.219094 \\
\hline $\mathrm{C}$ & 3.946287 & -0.942283 & 2.031434 \\
\hline $\mathrm{H}$ & 5.432706 & -1.015486 & 3.615842 \\
\hline B & 1.999546 & -0.099841 & 2.813441 \\
\hline $\mathrm{H}$ & 5.854437 & -1.954256 & 2.160110 \\
\hline $\mathrm{C}$ & -1.440402 & 1.449137 & 3.873526 \\
\hline $\mathrm{C}$ & -0.325746 & 0.543601 & 3.448594 \\
\hline 0 & 1.738353 & -1.434788 & 2.613524 \\
\hline $\mathrm{H}$ & -2.141796 & 1.577370 & 3.029545 \\
\hline $\mathrm{C}$ & 3.012282 & -2.095597 & 2.507219 \\
\hline $\mathrm{H}$ & -2.031620 & 0.964212 & 4.665713 \\
\hline $\mathrm{H}$ & 3.477801 & -1.782955 & 4.610655 \\
\hline $\mathrm{H}$ & 2.481851 & -2.940332 & 0.575968 \\
\hline $\mathrm{H}$ & -0.567901 & -0.514569 & 3.321700 \\
\hline $\mathrm{C}$ & 2.887772 & -3.256846 & 1.542580 \\
\hline $\mathrm{C}$ & 3.361771 & -2.609233 & 3.897323 \\
\hline $\mathrm{H}$ & 3.865325 & -3.731053 & 1.375658 \\
\hline $\mathrm{H}$ & 4.286872 & -3.200417 & 3.893850 \\
\hline $\mathrm{H}$ & 2.544519 & -3.248798 & 4.252449 \\
\hline $\mathrm{H}$ & 2.211535 & -4.013258 & 1.960084 \\
\hline $\mathrm{H}$ & -0.476156 & 2.703508 & 5.337808 \\
\hline $\mathrm{H}$ & -1.758963 & 3.518216 & 4.438794 \\
\hline
\end{tabular}

SCF energy: -644.877763343

M06-D3 (Gas Phase)/6-31G(d) +LANL2DZ (Pd) opt. No imaginary frequency 


\section{Cartesian Coordinates of Optimized Taniaphos Species}

\begin{tabular}{|c|c|c|c|}
\hline \multicolumn{4}{|c|}{ TS-2STania_conf7 } \\
\hline $\mathrm{P}$ & -0.808579 & 0.024080 & 1.859302 \\
\hline $\mathrm{C}$ & 4.641604 & 0.181218 & 1.501922 \\
\hline $\mathrm{C}$ & 7.340547 & -0.572046 & 1.525147 \\
\hline $\mathrm{C}$ & 5.039927 & -1.058634 & 0.993262 \\
\hline $\mathrm{C}$ & 5.615842 & 1.032966 & 2.039998 \\
\hline $\mathrm{C}$ & 6.955201 & 0.660204 & 2.045514 \\
\hline $\mathrm{C}$ & 6.380051 & -1.432765 & 1.003344 \\
\hline $\mathrm{H}$ & 4.309418 & -1.756104 & 0.584442 \\
\hline $\mathrm{H}$ & 5.328338 & 1.993120 & 2.468591 \\
\hline $\mathrm{H}$ & 7.698913 & 1.332269 & 2.468560 \\
\hline $\mathrm{H}$ & 6.669753 & -2.404717 & 0.608247 \\
\hline $\mathrm{H}$ & 8.388004 & -0.865881 & 1.536921 \\
\hline $\mathrm{C}$ & -2.182478 & -0.036858 & 0.640393 \\
\hline $\mathrm{C}$ & -4.280487 & -0.112286 & -1.218228 \\
\hline $\mathrm{C}$ & -3.106474 & 1.021003 & 0.564368 \\
\hline $\mathrm{C}$ & -2.348880 & -1.143551 & -0.198790 \\
\hline $\mathrm{C}$ & -3.394428 & -1.181046 & -1.118514 \\
\hline $\mathrm{C}$ & -4.140726 & 0.986066 & -0.370191 \\
\hline $\mathrm{H}$ & -1.682292 & -1.998503 & -0.119439 \\
\hline $\mathrm{H}$ & -3.513947 & -2.057896 & -1.752006 \\
\hline $\mathrm{H}$ & -4.854711 & 1.806689 & -0.415689 \\
\hline $\mathrm{H}$ & -5.094554 & -0.142045 & -1.939487 \\
\hline $\mathrm{C}$ & -1.498281 & -0.642061 & 3.400638 \\
\hline $\mathrm{C}$ & -2.406304 & -1.885827 & 5.722352 \\
\hline $\mathrm{C}$ & -2.827962 & -1.063590 & 3.492809 \\
\hline $\mathrm{C}$ & -0.624903 & -0.846290 & 4.476588 \\
\hline $\mathrm{C}$ & -1.081572 & -1.459967 & 5.635714 \\
\hline $\mathrm{C}$ & -3.276750 & -1.684818 & 4.654813 \\
\hline $\mathrm{H}$ & -3.513047 & -0.925764 & 2.656911 \\
\hline $\mathrm{H}$ & 0.418502 & -0.539011 & 4.399234 \\
\hline $\mathrm{H}$ & -0.398639 & -1.616606 & 6.468871 \\
\hline $\mathrm{H}$ & -4.311009 & -2.015025 & 4.724296 \\
\hline $\mathrm{H}$ & -2.761182 & -2.376743 & 6.626133 \\
\hline $\mathrm{C}$ & -0.596655 & 1.830825 & 2.110513 \\
\hline $\mathrm{C}$ & -0.131773 & 4.556846 & 2.548829 \\
\hline $\mathrm{C}$ & -0.917694 & 2.419365 & 3.335461 \\
\hline $\mathrm{C}$ & -0.041375 & 2.625569 & 1.088003 \\
\hline $\mathrm{C}$ & 0.207386 & 3.977165 & 1.331361 \\
\hline $\mathrm{C}$ & -0.697348 & 3.776642 & 3.550104 \\
\hline $\mathrm{H}$ & -1.340660 & 1.811443 & 4.133690 \\
\hline $\mathrm{H}$ & 0.698271 & 4.579470 & 0.565011 \\
\hline $\mathrm{H}$ & -0.955737 & 4.219161 & 4.509697 \\
\hline $\mathrm{H}$ & 0.063310 & 5.613504 & 2.719474 \\
\hline $\mathrm{C}$ & 2.784189 & 1.717075 & 3.006610 \\
\hline $\mathrm{C}$ & 2.711125 & 3.034206 & 5.461570 \\
\hline C & 2.647188 & 0.987050 & 4.193727 \\
\hline $\mathrm{C}$ & 2.904588 & 3.105749 & 3.056690 \\
\hline $\mathrm{C}$ & 2.867521 & 3.760738 & 4.285523 \\
\hline $\mathrm{C}$ & 2.605589 & 1.644867 & 5.416176 \\
\hline $\mathrm{H}$ & 2.599318 & -0.105307 & 4.157484 \\
\hline $\mathrm{H}$ & 3.039647 & 3.677738 & 2.137418 \\
\hline $\mathrm{H}$ & 2.967904 & 4.844246 & 4.323056 \\
\hline $\mathrm{H}$ & 2.503814 & 1.073916 & 6.337349 \\
\hline $\mathrm{H}$ & 2.685561 & 3.550014 & 6.419309 \\
\hline $\mathrm{H}$ & -3.027458 & 1.867984 & 1.249263 \\
\hline $\mathrm{H}$ & 5.037236 & 2.340580 & -0.209244 \\
\hline $\mathrm{C}$ & 4.012043 & 2.516743 & -0.519088 \\
\hline $\mathrm{C}$ & 2.852910 & 1.917779 & 0.075290 \\
\hline $\mathrm{H}$ & 4.256467 & 3.824438 & -2.313526 \\
\hline $\mathrm{C}$ & 1.704053 & 2.352838 & -0.699544 \\
\hline $\mathrm{C}$ & 2.186999 & 3.204900 & -1.741117 \\
\hline $\mathrm{H}$ & 1.609325 & 3.671255 & -2.533109 \\
\hline $\mathrm{C}$ & 3.601737 & 3.294645 & -1.629904 \\
\hline $\mathrm{Fe}$ & 3.029481 & 1.344477 & -1.854837 \\
\hline $\mathrm{H}$ & 3.087577 & -1.367932 & -1.084167 \\
\hline $\mathrm{C}$ & 3.217752 & -0.696698 & -1.927811 \\
\hline $\mathrm{C}$ & 4.427415 & -0.062834 & -2.331880 \\
\hline $\mathrm{H}$ & 1.150072 & -0.602427 & -2.754162 \\
\hline $\mathrm{H}$ & 5.388557 & -0.145812 & -1.833584 \\
\hline $\mathrm{C}$ & 4.135156 & 0.750288 & -3.463521 \\
\hline
\end{tabular}

\begin{tabular}{|c|c|c|c|}
\hline $\mathrm{H}$ & 4.837480 & 1.393030 & -3.983544 \\
\hline C & 2.746665 & 0.626226 & -3.752490 \\
\hline $\mathrm{H}$ & 2.219129 & 1.138523 & -4.551346 \\
\hline C & 2.182922 & -0.273109 & -2.802328 \\
\hline $\mathrm{C}$ & 0.316185 & 2.013679 & -0.249287 \\
\hline $\mathrm{H}$ & 0.276580 & 0.917102 & -0.158157 \\
\hline $\mathrm{N}$ & -0.822064 & 2.282503 & -1.250674 \\
\hline $\mathrm{C}$ & -0.720472 & 1.462613 & -2.491863 \\
\hline $\mathrm{H}$ & -0.690854 & 0.408413 & -2.199137 \\
\hline $\mathrm{H}$ & 0.190149 & 1.731226 & -3.032946 \\
\hline $\mathrm{H}$ & -1.609187 & 1.647451 & -3.102195 \\
\hline $\mathrm{C}$ & -1.151150 & 3.706517 & -1.553343 \\
\hline $\mathrm{H}$ & -1.330182 & 4.240364 & -0.617986 \\
\hline $\mathrm{H}$ & -2.060165 & 3.716488 & -2.161735 \\
\hline $\mathrm{H}$ & -0.332813 & 4.171900 & -2.104994 \\
\hline $\mathrm{Pd}$ & 1.263456 & -1.083539 & 1.438764 \\
\hline $\mathrm{H}$ & -1.647974 & 1.910671 & -0.748751 \\
\hline $\mathrm{P}$ & 2.890432 & 0.743809 & 1.469977 \\
\hline $\mathrm{C}$ & 0.254844 & -2.899644 & 1.152534 \\
\hline C & -0.693739 & -3.422730 & 1.937095 \\
\hline $\mathrm{H}$ & -0.825709 & -3.105831 & 2.973100 \\
\hline C & 0.494678 & -3.414169 & -0.242869 \\
\hline $\mathrm{H}$ & 0.142565 & -2.691379 & -1.000360 \\
\hline $\mathrm{H}$ & 1.568707 & -3.559521 & -0.430508 \\
\hline $\mathrm{C}$ & -0.231571 & -4.747379 & -0.401948 \\
\hline $\mathrm{H}$ & 0.296397 & -5.520173 & 0.187622 \\
\hline $\mathrm{H}$ & -0.262728 & -5.070844 & -1.447507 \\
\hline 0 & -1.566831 & -4.622947 & 0.035774 \\
\hline $\mathrm{C}$ & -1.632213 & -4.477383 & 1.427531 \\
\hline $\mathrm{H}$ & -2.666413 & -4.193302 & 1.675166 \\
\hline $\mathrm{H}$ & -1.436090 & -5.442783 & 1.936308 \\
\hline O & 2.072521 & -2.944914 & 3.584197 \\
\hline B & 2.130338 & -3.108892 & 2.232002 \\
\hline 0 & 2.617925 & -4.308019 & 1.806715 \\
\hline $\mathrm{C}$ & 3.189164 & -4.928919 & 3.000033 \\
\hline C & 2.367394 & -4.262627 & 4.147421 \\
\hline C & 3.120538 & -4.061966 & 5.442649 \\
\hline $\mathrm{H}$ & 3.998384 & -3.420872 & 5.311055 \\
\hline $\mathrm{H}$ & 3.449229 & -5.026246 & 5.852772 \\
\hline $\mathrm{H}$ & 2.462028 & -3.591151 & 6.183831 \\
\hline C & 1.039899 & -4.953033 & 4.409689 \\
\hline $\mathrm{H}$ & 0.500561 & -5.167488 & 3.478087 \\
\hline $\mathrm{H}$ & 0.410667 & -4.302150 & 5.031619 \\
\hline $\mathrm{H}$ & 1.184435 & -5.899590 & 4.944586 \\
\hline C & 4.659865 & -4.544309 & 3.016004 \\
\hline $\mathrm{H}$ & 4.795709 & -3.457158 & 3.106605 \\
\hline $\mathrm{H}$ & 5.120900 & -4.868496 & 2.074904 \\
\hline $\mathrm{H}$ & 5.194811 & -5.030461 & 3.840942 \\
\hline $\mathrm{C}$ & 3.029627 & -6.427867 & 2.887399 \\
\hline $\mathrm{H}$ & 1.990175 & -6.716793 & 2.697722 \\
\hline $\mathrm{H}$ & 3.367540 & -6.921724 & 3.808384 \\
\hline $\mathrm{H}$ & 3.642293 & -6.806000 & 2.060634 \\
\hline $\mathrm{H}$ & 2.619556 & -2.137147 & 1.473048 \\
\hline
\end{tabular}

SCF energy: -3329.867288360

M06-D3 (Gas Phase) /6-31G(d)+SDD (Pd, Fe) opt. 1 imaginary frequency: $198 \mathrm{i} \mathrm{cm-1}$ 


\begin{tabular}{|c|c|c|c|}
\hline \multicolumn{4}{|c|}{ TS-2RTania_conf3 } \\
\hline $\mathrm{P}$ & 0.167424 & 2.081016 & 0.289975 \\
\hline $\mathrm{C}$ & 0.430956 & -3.323278 & 0.778042 \\
\hline $\mathrm{C}$ & -0.193411 & -6.055259 & 0.776067 \\
\hline $\mathrm{C}$ & -0.318436 & -3.882429 & -0.260491 \\
\hline C & 0.841827 & -4.150701 & 1.832725 \\
\hline $\mathrm{C}$ & 0.536645 & -5.506667 & 1.827299 \\
\hline $\mathrm{C}$ & -0.626234 & -5.239709 & -0.264020 \\
\hline $\mathrm{H}$ & -0.686091 & -3.264914 & -1.079361 \\
\hline $\mathrm{H}$ & 1.398120 & -3.732376 & 2.671783 \\
\hline $\mathrm{H}$ & 0.862415 & -6.134902 & 2.653726 \\
\hline $\mathrm{H}$ & -1.214347 & -5.655710 & -1.079918 \\
\hline $\mathrm{H}$ & -0.436270 & -7.115759 & 0.775523 \\
\hline $\mathrm{C}$ & 0.824530 & 3.242992 & -0.971799 \\
\hline C & 1.895551 & 4.964368 & -2.904220 \\
\hline $\mathrm{C}$ & 1.678800 & 4.294457 & -0.593572 \\
\hline C & 0.508108 & 3.074183 & -2.324734 \\
\hline C & 1.036019 & 3.936990 & -3.283178 \\
\hline $\mathrm{C}$ & 2.216153 & 5.144600 & -1.558358 \\
\hline $\mathrm{H}$ & -0.157443 & 2.264727 & -2.629017 \\
\hline $\mathrm{H}$ & 0.770440 & 3.804484 & -4.330124 \\
\hline $\mathrm{H}$ & 2.869470 & 5.961156 & -1.256100 \\
\hline $\mathrm{H}$ & 2.307445 & 5.635674 & -3.654969 \\
\hline $\mathrm{C}$ & -1.187879 & 2.935362 & 1.145052 \\
\hline $\mathrm{C}$ & -3.319384 & 4.139811 & 2.474126 \\
\hline C & -1.395514 & 4.313082 & 1.028533 \\
\hline $\mathrm{C}$ & -2.054646 & 2.162262 & 1.926585 \\
\hline C & -3.110745 & 2.768181 & 2.596567 \\
\hline C & -2.464144 & 4.909320 & 1.689665 \\
\hline $\mathrm{H}$ & -0.738908 & 4.923756 & 0.409521 \\
\hline $\mathrm{H}$ & -1.921470 & 1.082428 & 1.989458 \\
\hline $\mathrm{H}$ & -3.778899 & 2.164463 & 3.209216 \\
\hline $\mathrm{H}$ & -2.629634 & 5.979931 & 1.590515 \\
\hline $\mathrm{H}$ & -4.153596 & 4.611504 & 2.989249 \\
\hline C & 1.548931 & 2.027690 & 1.501521 \\
\hline $\mathrm{C}$ & 3.610032 & 1.863247 & 3.390602 \\
\hline $\mathrm{C}$ & 1.372560 & 2.527870 & 2.793678 \\
\hline $\mathrm{C}$ & 2.778195 & 1.434837 & 1.154349 \\
\hline $\mathrm{C}$ & 3.785462 & 1.336306 & 2.116215 \\
\hline $\mathrm{C}$ & 2.401437 & 2.460623 & 3.727904 \\
\hline $\mathrm{H}$ & 0.421009 & 2.975369 & 3.076259 \\
\hline $\mathrm{H}$ & 4.714672 & 0.819611 & 1.870940 \\
\hline $\mathrm{H}$ & 2.248048 & 2.862070 & 4.727328 \\
\hline $\mathrm{H}$ & 4.410301 & 1.787969 & 4.123780 \\
\hline C & 0.813726 & -1.164691 & 2.581551 \\
\hline $\mathrm{C}$ & 0.438033 & -0.620981 & 5.288836 \\
\hline C & -0.476528 & -0.953341 & 3.084724 \\
\hline C & 1.909501 & -1.126278 & 3.443008 \\
\hline C & 1.717739 & -0.855314 & 4.795878 \\
\hline C & -0.661051 & -0.675625 & 4.432830 \\
\hline $\mathrm{H}$ & -1.341323 & -1.026225 & 2.418019 \\
\hline $\mathrm{H}$ & 2.914058 & -1.316171 & 3.062276 \\
\hline $\mathrm{H}$ & 2.574167 & -0.830281 & 5.467815 \\
\hline $\mathrm{H}$ & -1.665941 & -0.511498 & 4.819311 \\
\hline $\mathrm{H}$ & 0.293639 & -0.409194 & 6.346338 \\
\hline $\mathrm{H}$ & 1.922979 & 4.448695 & 0.459494 \\
\hline $\mathrm{H}$ & 3.198590 & -3.766945 & 0.746737 \\
\hline C & 3.528007 & -2.781180 & 0.434295 \\
\hline $\mathrm{C}$ & 2.699786 & -1.612411 & 0.366845 \\
\hline $\mathrm{H}$ & 5.640823 & -3.146049 & -0.192450 \\
\hline $\mathrm{C}$ & 3.515437 & -0.545872 & -0.187439 \\
\hline C & 4.815188 & -1.083971 & -0.438265 \\
\hline $\mathrm{H}$ & 5.662221 & -0.575229 & -0.888986 \\
\hline C & 4.813785 & -2.455794 & -0.064880 \\
\hline $\mathrm{Fe}$ & 3.373856 & -2.105505 & -1.474132 \\
\hline $\mathrm{H}$ & 0.727834 & -2.515113 & -2.363358 \\
\hline C & 1.769275 & -2.597753 & -2.653571 \\
\hline $\mathrm{C}$ & 2.611958 & -3.726906 & -2.453227 \\
\hline $\mathrm{H}$ & 2.155127 & -0.599646 & -3.560092 \\
\hline $\mathrm{H}$ & 2.331295 & -4.650625 & -1.956538 \\
\hline $\mathrm{C}$ & 3.902383 & -3.401359 & -2.958785 \\
\hline $\mathrm{H}$ & 4.780138 & -4.037750 & -2.921950 \\
\hline C & 3.858422 & -2.070060 & -3.462191 \\
\hline
\end{tabular}

\begin{tabular}{|c|c|c|c|}
\hline $\mathrm{H}$ & 4.693890 & -1.536797 & -3.905603 \\
\hline $\mathrm{C}$ & 2.535182 & -1.575296 & -3.272574 \\
\hline $\mathrm{C}$ & 2.991216 & 0.855721 & -0.227974 \\
\hline $\mathrm{H}$ & 2.023073 & 0.809126 & -0.750068 \\
\hline $\mathrm{N}$ & 3.766368 & 1.863366 & -1.098365 \\
\hline $\mathrm{C}$ & 3.836651 & 1.468588 & -2.534265 \\
\hline $\mathrm{H}$ & 2.816724 & 1.310907 & -2.894602 \\
\hline $\mathrm{H}$ & 4.415361 & 0.546922 & -2.628833 \\
\hline $\mathrm{H}$ & 4.303300 & 2.283764 & -3.094437 \\
\hline $\mathrm{C}$ & 5.092908 & 2.327788 & -0.595437 \\
\hline $\mathrm{H}$ & 4.975262 & 2.731200 & 0.412088 \\
\hline $\mathrm{H}$ & 5.447452 & 3.114293 & -1.267917 \\
\hline $\mathrm{H}$ & 5.802010 & 1.497670 & -0.591106 \\
\hline $\mathrm{Pd}$ & -0.543484 & -0.060807 & -0.461495 \\
\hline $\mathrm{H}$ & 3.154273 & 2.696957 & -1.065510 \\
\hline $\mathrm{P}$ & 0.929706 & -1.553044 & 0.804208 \\
\hline $\mathrm{C}$ & -2.001376 & 0.759230 & -1.755394 \\
\hline C & -1.896957 & 0.261708 & -2.999781 \\
\hline $\mathrm{H}$ & -1.359054 & -0.666926 & -3.210049 \\
\hline $\mathrm{C}$ & -2.852255 & 1.967415 & -1.491826 \\
\hline $\mathrm{H}$ & -3.403767 & 1.873875 & -0.544413 \\
\hline $\mathrm{H}$ & -2.227979 & 2.869268 & -1.387743 \\
\hline C & -3.810996 & 2.162495 & -2.661109 \\
\hline $\mathrm{H}$ & -4.334833 & 3.121639 & -2.592568 \\
\hline $\mathrm{H}$ & -4.567843 & 1.353532 & -2.677547 \\
\hline $\mathrm{O}$ & -3.088776 & 2.169331 & -3.871488 \\
\hline $\mathrm{C}$ & -2.590277 & 0.898436 & -4.168408 \\
\hline $\mathrm{H}$ & -3.400121 & 0.217807 & -4.502926 \\
\hline $\mathrm{H}$ & -1.899021 & 1.008122 & -5.015731 \\
\hline O & -3.101296 & -0.715178 & 0.570746 \\
\hline B & -2.668524 & -1.022995 & -0.689866 \\
\hline $\mathrm{O}$ & -3.567971 & -1.713498 & -1.443878 \\
\hline $\mathrm{C}$ & -4.573738 & -2.196581 & -0.500240 \\
\hline C & -4.499440 & -1.132484 & 0.639255 \\
\hline C & -4.750022 & -1.659483 & 2.035171 \\
\hline $\mathrm{H}$ & -4.041887 & -2.449206 & 2.309162 \\
\hline $\mathrm{H}$ & -5.769677 & -2.056487 & 2.126844 \\
\hline $\mathrm{H}$ & -4.643970 & -0.840514 & 2.759852 \\
\hline C & -5.351289 & 0.096504 & 0.378169 \\
\hline $\mathrm{H}$ & -5.210982 & 0.481429 & -0.639843 \\
\hline $\mathrm{H}$ & -5.069291 & 0.888154 & 1.085515 \\
\hline $\mathrm{H}$ & -6.417541 & -0.119497 & 0.516923 \\
\hline $\mathrm{C}$ & -4.121208 & -3.577481 & -0.055116 \\
\hline $\mathrm{H}$ & -3.160580 & -3.540726 & 0.479180 \\
\hline $\mathrm{H}$ & -3.993948 & -4.211572 & -0.941173 \\
\hline $\mathrm{H}$ & -4.862697 & -4.052633 & 0.598810 \\
\hline $\mathrm{C}$ & -5.903208 & -2.274175 & -1.215237 \\
\hline $\mathrm{H}$ & -6.166965 & -1.325201 & -1.694215 \\
\hline $\mathrm{H}$ & -6.702439 & -2.547091 & -0.512994 \\
\hline $\mathrm{H}$ & -5.862629 & -3.045441 & -1.993150 \\
\hline $\mathrm{H}$ & -1.437957 & -1.477698 & -0.881482 \\
\hline
\end{tabular}

SCF energy: -3329.862924440

M06-D3 (Gas Phase)/6-31G(d)+SDD (Pd, Fe) opt. 1 imaginary frequency: $\quad 204 \mathrm{i} \mathrm{cm-1}$ 


\begin{tabular}{|c|c|c|c|}
\hline \multicolumn{4}{|c|}{ TS-2RTania_conf2 } \\
\hline $\mathrm{P}$ & 0.533534 & -1.619884 & 1.054607 \\
\hline $\mathrm{P}$ & 1.041452 & 1.903985 & 0.265884 \\
\hline $\mathrm{C}$ & -0.561089 & -3.038505 & 1.477208 \\
\hline $\mathrm{C}$ & -2.120281 & -5.276581 & 2.128379 \\
\hline C & -1.648390 & -3.408276 & 0.678161 \\
\hline $\mathrm{C}$ & -0.272260 & -3.804258 & 2.615232 \\
\hline $\mathrm{C}$ & -1.045704 & -4.915043 & 2.934779 \\
\hline $\mathrm{C}$ & -2.420350 & -4.519942 & 0.999536 \\
\hline $\mathrm{H}$ & -1.924593 & -2.830923 & -0.202080 \\
\hline $\mathrm{H}$ & 0.560035 & -3.534248 & 3.263952 \\
\hline $\mathrm{H}$ & -0.808938 & -5.495422 & 3.824035 \\
\hline $\mathrm{H}$ & -3.265143 & -4.786694 & 0.366549 \\
\hline $\mathrm{H}$ & -2.728173 & -6.141933 & 2.383821 \\
\hline C & 1.627099 & 2.930346 & -1.135160 \\
\hline $\mathrm{C}$ & 2.499726 & 4.491218 & -3.288688 \\
\hline C & 2.655160 & 3.868359 & -0.962250 \\
\hline C & 1.063599 & 2.765466 & -2.404595 \\
\hline $\mathrm{C}$ & 1.499826 & 3.541277 & -3.476872 \\
\hline C & 3.078529 & 4.652023 & -2.030318 \\
\hline $\mathrm{H}$ & 0.266760 & 2.033696 & -2.546164 \\
\hline $\mathrm{H}$ & 1.044407 & 3.412074 & -4.457561 \\
\hline $\mathrm{H}$ & 3.866824 & 5.387476 & -1.882149 \\
\hline $\mathrm{H}$ & 2.831336 & 5.107114 & -4.122098 \\
\hline $\mathrm{C}$ & 0.260004 & 3.023244 & 1.477014 \\
\hline C & -1.064933 & 4.635585 & 3.337120 \\
\hline $\mathrm{C}$ & 0.684322 & 4.338976 & 1.701557 \\
\hline C & -0.849182 & 2.536057 & 2.179602 \\
\hline C & -1.505394 & 3.336031 & 3.106650 \\
\hline C & 0.025455 & 5.136432 & 2.630185 \\
\hline $\mathrm{H}$ & 1.519974 & 4.759176 & 1.145569 \\
\hline $\mathrm{H}$ & -1.236796 & 1.539682 & 1.963160 \\
\hline $\mathrm{H}$ & -2.373381 & 2.946595 & 3.635305 \\
\hline $\mathrm{H}$ & 0.360958 & 6.158010 & 2.795356 \\
\hline $\mathrm{H}$ & -1.580731 & 5.267033 & 4.057535 \\
\hline $\mathrm{C}$ & 2.635558 & 1.345149 & 1.011967 \\
\hline $\mathrm{C}$ & 5.035698 & 0.509181 & 2.210177 \\
\hline $\mathrm{C}$ & 3.048855 & 1.869852 & 2.240555 \\
\hline $\mathrm{C}$ & 3.436282 & 0.366755 & 0.391296 \\
\hline C & 4.614889 & -0.053014 & 1.012008 \\
\hline C & 4.244481 & 1.471169 & 2.827841 \\
\hline $\mathrm{H}$ & 2.420868 & 2.597167 & 2.753243 \\
\hline $\mathrm{H}$ & 5.211692 & -0.849674 & 0.561055 \\
\hline $\mathrm{H}$ & 4.546791 & 1.901954 & 3.779992 \\
\hline $\mathrm{H}$ & 5.964151 & 0.178835 & 2.670848 \\
\hline $\mathrm{C}$ & 1.227078 & -1.178685 & 2.680352 \\
\hline C & 2.092632 & -0.567598 & 5.258652 \\
\hline C & 0.407291 & -0.443660 & 3.543721 \\
\hline C & 2.473684 & -1.624351 & 3.122155 \\
\hline C & 2.901857 & -1.318468 & 4.411312 \\
\hline C & 0.841827 & -0.131478 & 4.825428 \\
\hline $\mathrm{H}$ & -0.587142 & -0.133791 & 3.216095 \\
\hline $\mathrm{H}$ & 3.108641 & -2.220713 & 2.465501 \\
\hline $\mathrm{H}$ & 3.872079 & -1.672934 & 4.755637 \\
\hline $\mathrm{H}$ & 0.199852 & 0.442813 & 5.490660 \\
\hline $\mathrm{H}$ & 2.431001 & -0.332513 & 6.265787 \\
\hline $\mathrm{H}$ & 3.151557 & 3.970923 & 0.003683 \\
\hline $\mathrm{H}$ & 1.546700 & -4.550949 & 0.634618 \\
\hline C & 2.144871 & -3.806901 & 0.117485 \\
\hline C & 1.886726 & -2.397463 & 0.122052 \\
\hline $\mathrm{H}$ & 3.648547 & -5.041816 & -0.981335 \\
\hline C & 2.856735 & -1.777610 & -0.757608 \\
\hline C & 3.696103 & -2.826250 & -1.262705 \\
\hline $\mathrm{H}$ & 4.509622 & -2.741205 & -1.980603 \\
\hline $\mathrm{C}$ & 3.247537 & -4.065388 & -0.731786 \\
\hline $\mathrm{Fe}$ & 1.741202 & -3.132607 & -1.762865 \\
\hline $\mathrm{H}$ & -0.947048 & -2.276672 & -1.947145 \\
\hline $\mathrm{C}$ & -0.169438 & -2.836833 & -2.457554 \\
\hline C & 0.103723 & -4.226253 & -2.315137 \\
\hline $\mathrm{H}$ & 0.816938 & -1.247021 & -3.673666 \\
\hline $\mathrm{H}$ & -0.413207 & -4.912668 & -1.651852 \\
\hline $\mathrm{C}$ & 1.214786 & -4.539758 & -3.147273 \\
\hline $\mathrm{H}$ & 1.690951 & -5.510373 & -3.236156 \\
\hline
\end{tabular}

\begin{tabular}{|c|c|c|c|}
\hline C & 1.631465 & -3.344022 & -3.797616 \\
\hline $\mathrm{H}$ & 2.462809 & -3.263205 & -4.491756 \\
\hline C & 0.773778 & -2.289216 & -3.367075 \\
\hline C & 3.047227 & -0.287997 & -0.916596 \\
\hline $\mathrm{H}$ & 2.137340 & 0.173296 & -1.328494 \\
\hline $\mathrm{N}$ & 4.122040 & -0.016914 & -1.990532 \\
\hline $\mathrm{C}$ & 4.684193 & 1.370702 & -1.993582 \\
\hline $\mathrm{H}$ & 5.092969 & 1.610217 & -1.010639 \\
\hline $\mathrm{H}$ & 3.881045 & 2.066792 & -2.247609 \\
\hline $\mathrm{H}$ & 5.470755 & 1.410622 & -2.751862 \\
\hline C & 3.630498 & -0.338872 & -3.366392 \\
\hline $\mathrm{H}$ & 3.192003 & -1.338686 & -3.365826 \\
\hline $\mathrm{H}$ & 4.469583 & -0.276088 & -4.064613 \\
\hline $\mathrm{H}$ & 2.874601 & 0.409737 & -3.627072 \\
\hline $\mathrm{Pd}$ & -0.531781 & 0.178419 & -0.171286 \\
\hline $\mathrm{C}$ & -1.830353 & 1.378687 & -1.357993 \\
\hline $\mathrm{C}$ & -2.132376 & 0.911892 & -2.584494 \\
\hline $\mathrm{H}$ & -1.852339 & -0.095529 & -2.903163 \\
\hline C & -2.236778 & 2.776995 & -0.971205 \\
\hline $\mathrm{H}$ & -3.038414 & 2.734108 & -0.218722 \\
\hline $\mathrm{H}$ & -1.410752 & 3.321682 & -0.490956 \\
\hline C & -2.687603 & 3.558423 & -2.201224 \\
\hline $\mathrm{H}$ & -1.809945 & 3.848036 & -2.811707 \\
\hline $\mathrm{H}$ & -3.213893 & 4.474952 & -1.915154 \\
\hline O & -3.581933 & 2.799122 & -2.980213 \\
\hline C & -2.949446 & 1.701155 & -3.564634 \\
\hline $\mathrm{H}$ & -3.736138 & 1.062447 & -3.989345 \\
\hline $\mathrm{H}$ & -2.300227 & 2.016167 & -4.409771 \\
\hline 0 & -3.341405 & 0.464372 & 0.743632 \\
\hline B & -2.860143 & -0.168287 & -0.366896 \\
\hline O & -3.767488 & -0.949585 & -1.025659 \\
\hline C & -5.067572 & -0.605220 & -0.456704 \\
\hline C & -4.681459 & -0.074550 & 0.961729 \\
\hline C & -4.526513 & -1.169819 & 2.003520 \\
\hline $\mathrm{H}$ & -3.874354 & -1.980252 & 1.648726 \\
\hline $\mathrm{H}$ & -5.494554 & -1.600672 & 2.287035 \\
\hline $\mathrm{H}$ & -4.070019 & -0.740530 & 2.904508 \\
\hline C & -5.562934 & 1.038124 & 1.482412 \\
\hline $\mathrm{H}$ & -5.552647 & 1.911166 & 0.820717 \\
\hline $\mathrm{H}$ & -5.215985 & 1.355191 & 2.473709 \\
\hline $\mathrm{H}$ & -6.600486 & 0.693401 & 1.586392 \\
\hline $\mathrm{C}$ & -5.922718 & -1.852251 & -0.446518 \\
\hline $\mathrm{H}$ & -5.422302 & -2.683877 & 0.062113 \\
\hline $\mathrm{H}$ & -6.140781 & -2.162822 & -1.475031 \\
\hline $\mathrm{H}$ & -6.880190 & -1.659898 & 0.056081 \\
\hline $\mathrm{C}$ & -5.674307 & 0.453127 & -1.361654 \\
\hline $\mathrm{H}$ & -5.052778 & 1.357023 & -1.431425 \\
\hline $\mathrm{H}$ & -6.675526 & 0.746367 & -1.023109 \\
\hline $\mathrm{H}$ & -5.773528 & 0.037667 & -2.372285 \\
\hline $\mathrm{H}$ & -1.765439 & -0.941118 & -0.386839 \\
\hline $\mathrm{H}$ & 4.896764 & -0.655786 & -1.774622 \\
\hline
\end{tabular}

SCF energy: -3329.861266950

M06-D3 (Gas Phase)/6-31G(d)+SDD (Pd, Fe) opt. 1 imaginary frequency: $183 \mathrm{i} \mathrm{cm-1}$ 


\begin{tabular}{|c|c|c|c|}
\hline \multicolumn{4}{|c|}{ TS-2STania_conf 6} \\
\hline $\mathrm{P}$ & -0.753328 & 0.016899 & 1.863395 \\
\hline $\mathrm{C}$ & 4.657172 & 0.142396 & 1.397632 \\
\hline $\mathrm{C}$ & 7.350298 & -0.628234 & 1.370675 \\
\hline $\mathrm{C}$ & 5.043627 & -1.077102 & 0.833843 \\
\hline C & 5.640084 & 0.965264 & 1.964086 \\
\hline $\mathrm{C}$ & 6.976542 & 0.582783 & 1.946661 \\
\hline $\mathrm{C}$ & 6.381397 & -1.458900 & 0.816672 \\
\hline $\mathrm{H}$ & 4.302704 & -1.753580 & 0.409116 \\
\hline $\mathrm{H}$ & 5.361748 & 1.909597 & 2.431864 \\
\hline $\mathrm{H}$ & 7.727443 & 1.230925 & 2.393681 \\
\hline $\mathrm{H}$ & 6.662572 & -2.414803 & 0.378764 \\
\hline $\mathrm{H}$ & 8.395805 & -0.929108 & 1.363407 \\
\hline $\mathrm{C}$ & -2.072061 & -0.061882 & 0.595517 \\
\hline C & -4.068961 & -0.177600 & -1.359994 \\
\hline $\mathrm{C}$ & -3.136737 & 0.853606 & 0.616163 \\
\hline C & -2.023766 & -1.033843 & -0.407716 \\
\hline C & -3.022918 & -1.096603 & -1.377095 \\
\hline $\mathrm{C}$ & -4.125814 & 0.798029 & -0.363953 \\
\hline $\mathrm{H}$ & -1.195585 & -1.742526 & -0.423681 \\
\hline $\mathrm{H}$ & -2.982808 & -1.865797 & -2.146641 \\
\hline $\mathrm{H}$ & -4.954431 & 1.503340 & -0.337707 \\
\hline $\mathrm{H}$ & -4.849302 & -0.226411 & -2.116652 \\
\hline C & -1.480997 & -0.648577 & 3.388591 \\
\hline $\mathrm{C}$ & -2.430120 & -1.876861 & 5.704564 \\
\hline C & -2.772673 & -1.181880 & 3.419106 \\
\hline $\mathrm{C}$ & -0.661174 & -0.742325 & 4.522003 \\
\hline C & -1.142051 & -1.343927 & 5.678065 \\
\hline C & -3.241965 & -1.795912 & 4.577021 \\
\hline $\mathrm{H}$ & -3.412470 & -1.136963 & 2.538455 \\
\hline $\mathrm{H}$ & 0.359122 & -0.355939 & 4.497315 \\
\hline $\mathrm{H}$ & -0.504363 & -1.406375 & 6.558603 \\
\hline $\mathrm{H}$ & -4.246907 & -2.212160 & 4.596739 \\
\hline $\mathrm{H}$ & -2.801125 & -2.357507 & 6.607338 \\
\hline C & -0.569282 & 1.828156 & 2.137152 \\
\hline $\mathrm{C}$ & -0.072834 & 4.541386 & 2.641094 \\
\hline $\mathrm{C}$ & -0.885200 & 2.395142 & 3.374136 \\
\hline $\mathrm{C}$ & -0.014034 & 2.645100 & 1.132170 \\
\hline $\mathrm{C}$ & 0.254389 & 3.986621 & 1.409316 \\
\hline $\mathrm{C}$ & -0.651768 & 3.744559 & 3.620968 \\
\hline $\mathrm{H}$ & -1.313383 & 1.776061 & 4.160219 \\
\hline $\mathrm{H}$ & 0.748871 & 4.601678 & 0.655636 \\
\hline $\mathrm{H}$ & -0.907932 & 4.166297 & 4.590438 \\
\hline $\mathrm{H}$ & 0.138870 & 5.590225 & 2.838005 \\
\hline C & 2.825960 & 1.641603 & 2.966058 \\
\hline $\mathrm{C}$ & 2.803532 & 2.900067 & 5.453225 \\
\hline C & 2.701148 & 0.882453 & 4.137173 \\
\hline C & 2.959747 & 3.027591 & 3.048084 \\
\hline C & 2.946944 & 3.653497 & 4.292473 \\
\hline C & 2.685770 & 1.513243 & 5.375078 \\
\hline $\mathrm{H}$ & 2.634049 & -0.208513 & 4.079294 \\
\hline $\mathrm{H}$ & 3.086928 & 3.620439 & 2.140851 \\
\hline $\mathrm{H}$ & 3.056906 & 4.734973 & 4.354169 \\
\hline $\mathrm{H}$ & 2.593708 & 0.919745 & 6.283749 \\
\hline $\mathrm{H}$ & 2.797790 & 3.392869 & 6.423362 \\
\hline $\mathrm{H}$ & -3.199234 & 1.600271 & 1.410625 \\
\hline $\mathrm{H}$ & 5.043320 & 2.266538 & -0.317128 \\
\hline C & 4.015522 & 2.479004 & -0.594082 \\
\hline C & 2.856507 & 1.915317 & 0.033834 \\
\hline $\mathrm{H}$ & 4.246698 & 3.794474 & -2.384563 \\
\hline $\mathrm{C}$ & 1.698295 & 2.395251 & -0.700090 \\
\hline C & 2.177214 & 3.240425 & -1.749757 \\
\hline $\mathrm{H}$ & 1.591456 & 3.737608 & -2.516831 \\
\hline C & 3.596934 & 3.279921 & -1.684804 \\
\hline $\mathrm{Fe}$ & 2.946439 & 1.352041 & -1.906295 \\
\hline $\mathrm{H}$ & 2.972655 & -1.364133 & -1.139729 \\
\hline C & 3.074618 & -0.694186 & -1.988681 \\
\hline $\mathrm{C}$ & 4.276271 & -0.094154 & -2.461848 \\
\hline $\mathrm{H}$ & 0.965176 & -0.538976 & -2.689406 \\
\hline $\mathrm{H}$ & 5.261753 & -0.205385 & -2.020197 \\
\hline $\mathrm{C}$ & 3.942731 & 0.728277 & -3.575014 \\
\hline $\mathrm{H}$ & 4.632032 & 1.350640 & -4.135595 \\
\hline C & 2.536959 & 0.644016 & -3.783692 \\
\hline
\end{tabular}

\begin{tabular}{|c|c|c|c|}
\hline $\mathrm{H}$ & 1.980647 & 1.169985 & -4.553609 \\
\hline $\mathrm{C}$ & 2.003204 & -0.239289 & -2.800919 \\
\hline C & 0.310840 & 2.075391 & -0.230576 \\
\hline $\mathrm{H}$ & 0.242034 & 0.977907 & -0.185354 \\
\hline $\mathrm{N}$ & -0.839636 & 2.425920 & -1.200416 \\
\hline C & -0.765302 & 1.695605 & -2.499959 \\
\hline $\mathrm{H}$ & -0.747324 & 0.624010 & -2.286995 \\
\hline $\mathrm{H}$ & 0.141634 & 1.991071 & -3.032205 \\
\hline $\mathrm{H}$ & -1.657946 & 1.937435 & -3.083630 \\
\hline C & -1.132986 & 3.876097 & -1.412489 \\
\hline $\mathrm{H}$ & -1.333803 & 4.349290 & -0.450199 \\
\hline $\mathrm{H}$ & -2.017366 & 3.949240 & -2.051082 \\
\hline $\mathrm{H}$ & -0.284195 & 4.357968 & -1.900570 \\
\hline $\mathrm{Pd}$ & 1.289444 & -1.111482 & 1.411486 \\
\hline $\mathrm{H}$ & -1.665399 & 2.038461 & -0.712562 \\
\hline $\mathrm{P}$ & 2.908071 & 0.710150 & 1.401211 \\
\hline C & 0.342181 & -2.995270 & 1.330724 \\
\hline C & -0.499215 & -3.515879 & 2.231192 \\
\hline $\mathrm{H}$ & -0.652775 & -3.056596 & 3.208334 \\
\hline C & 0.548309 & -3.658918 & -0.006438 \\
\hline $\mathrm{H}$ & 0.607917 & -2.924198 & -0.828131 \\
\hline $\mathrm{H}$ & 1.509728 & -4.196924 & -0.009488 \\
\hline $\mathrm{C}$ & -0.598369 & -4.634385 & -0.272118 \\
\hline $\mathrm{H}$ & -0.374477 & -5.284133 & -1.124831 \\
\hline $\mathrm{H}$ & -1.529816 & -4.077533 & -0.502056 \\
\hline 0 & -0.800510 & -5.470880 & 0.835747 \\
\hline $\mathrm{C}$ & -1.265984 & -4.774634 & 1.953045 \\
\hline $\mathrm{H}$ & -2.338689 & -4.503393 & 1.840360 \\
\hline $\mathrm{H}$ & -1.205560 & -5.462771 & 2.807097 \\
\hline 0 & 2.097301 & -2.540929 & 3.713482 \\
\hline B & 2.197287 & -2.983875 & 2.421871 \\
\hline 0 & 2.829546 & -4.179493 & 2.272178 \\
\hline $\mathrm{C}$ & 3.470530 & -4.432163 & 3.563020 \\
\hline $\mathrm{C}$ & 2.543780 & -3.655439 & 4.545849 \\
\hline $\mathrm{C}$ & 3.224630 & -3.069390 & 5.762174 \\
\hline $\mathrm{H}$ & 4.027032 & -2.375304 & 5.487894 \\
\hline $\mathrm{H}$ & 3.646864 & -3.859975 & 6.396486 \\
\hline $\mathrm{H}$ & 2.486426 & -2.517802 & 6.361090 \\
\hline $\mathrm{C}$ & 1.313413 & -4.446980 & 4.954137 \\
\hline $\mathrm{H}$ & 0.830252 & -4.920881 & 4.090182 \\
\hline $\mathrm{H}$ & 0.589133 & -3.772152 & 5.429846 \\
\hline $\mathrm{H}$ & 1.569469 & -5.233821 & 5.673987 \\
\hline $\mathrm{C}$ & 4.867165 & -3.838119 & 3.472640 \\
\hline $\mathrm{H}$ & 4.840715 & -2.747697 & 3.327080 \\
\hline $\mathrm{H}$ & 5.387401 & -4.282284 & 2.615252 \\
\hline $\mathrm{H}$ & 5.453361 & -4.053467 & 4.374574 \\
\hline $\mathrm{C}$ & 3.536969 & -5.924863 & 3.788029 \\
\hline $\mathrm{H}$ & 2.556170 & -6.400040 & 3.686200 \\
\hline $\mathrm{H}$ & 3.932704 & -6.143739 & 4.789087 \\
\hline $\mathrm{H}$ & 4.210581 & -6.381799 & 3.053836 \\
\hline $\mathrm{H}$ & 2.633977 & -2.175768 & 1.435814 \\
\hline
\end{tabular}

SCF energy: -3329.863482330

M06-D3 (Gas Phase) /6-31G(d) +SDD (Pd, Fe) opt. 1 imaginary frequency: $\quad 208 \mathrm{i} \mathrm{cm-1}$ 


\begin{tabular}{|c|c|c|c|}
\hline \multicolumn{4}{|c|}{ TS-2STania_conf 4} \\
\hline $\mathrm{P}$ & 1.660127 & 1.297456 & -0.030710 \\
\hline $\mathrm{P}$ & -1.954998 & 0.507441 & 0.473202 \\
\hline $\mathrm{C}$ & 3.427266 & 0.785339 & -0.057479 \\
\hline $\mathrm{C}$ & 6.143045 & 0.098988 & -0.128241 \\
\hline $\mathrm{C}$ & 3.840727 & -0.432625 & -0.606089 \\
\hline $\mathrm{C}$ & 4.396092 & 1.651823 & 0.466839 \\
\hline $\mathrm{C}$ & 5.743569 & 1.311193 & 0.427118 \\
\hline $\mathrm{C}$ & 5.189077 & -0.773523 & -0.642782 \\
\hline $\mathrm{H}$ & 3.115155 & -1.138326 & -1.009819 \\
\hline $\mathrm{H}$ & 4.098739 & 2.599197 & 0.916478 \\
\hline $\mathrm{H}$ & 6.483093 & 1.993476 & 0.841048 \\
\hline $\mathrm{H}$ & 5.490718 & -1.729397 & -1.067129 \\
\hline $\mathrm{H}$ & 7.197087 & -0.169575 & -0.151363 \\
\hline C & -3.433230 & 0.293354 & -0.577068 \\
\hline $\mathrm{C}$ & -5.710596 & -0.042445 & -2.158671 \\
\hline C & -4.627348 & 0.948706 & -0.243603 \\
\hline C & -3.392646 & -0.527896 & -1.706162 \\
\hline $\mathrm{C}$ & -4.527491 & -0.692806 & -2.497718 \\
\hline C & -5.761388 & 0.775613 & -1.029653 \\
\hline $\mathrm{H}$ & -2.472727 & -1.054099 & -1.955799 \\
\hline $\mathrm{H}$ & -4.490990 & -1.349206 & -3.364880 \\
\hline $\mathrm{H}$ & -6.689012 & 1.276541 & -0.759781 \\
\hline $\mathrm{H}$ & -6.601071 & -0.180217 & -2.768581 \\
\hline $\mathrm{C}$ & -2.419505 & -0.144261 & 2.110736 \\
\hline C & -2.900218 & -1.386931 & 4.565298 \\
\hline $\mathrm{C}$ & -3.641784 & -0.781505 & 2.349031 \\
\hline C & -1.438640 & -0.141892 & 3.110406 \\
\hline C & -1.680560 & -0.753852 & 4.333032 \\
\hline C & -3.877781 & -1.397549 & 3.574787 \\
\hline $\mathrm{H}$ & -4.405303 & -0.825794 & 1.573996 \\
\hline $\mathrm{H}$ & -0.478441 & 0.340102 & 2.928474 \\
\hline $\mathrm{H}$ & -0.910964 & -0.742894 & 5.102956 \\
\hline $\mathrm{H}$ & -4.830074 & -1.892732 & 3.752515 \\
\hline $\mathrm{H}$ & -3.088517 & -1.873882 & 5.519924 \\
\hline $\mathrm{C}$ & -1.841506 & 2.340893 & 0.663837 \\
\hline C & -1.557981 & 5.111467 & 1.036584 \\
\hline $\mathrm{C}$ & -2.144311 & 2.937259 & 1.892331 \\
\hline $\mathrm{C}$ & -1.388525 & 3.157732 & -0.389751 \\
\hline $\mathrm{C}$ & -1.232456 & 4.529609 & -0.182021 \\
\hline C & -2.017025 & 4.309973 & 2.075662 \\
\hline $\mathrm{H}$ & -2.484033 & 2.319026 & 2.721674 \\
\hline $\mathrm{H}$ & -0.836498 & 5.156827 & -0.984777 \\
\hline $\mathrm{H}$ & -2.266415 & 4.750078 & 3.038711 \\
\hline $\mathrm{H}$ & -1.439076 & 6.183609 & 1.177783 \\
\hline $\mathrm{C}$ & 1.600758 & 2.341282 & 1.461348 \\
\hline $\mathrm{C}$ & 1.596437 & 3.810082 & 3.830611 \\
\hline C & 1.642836 & 1.684053 & 2.697309 \\
\hline $\mathrm{C}$ & 1.588723 & 3.735333 & 1.418707 \\
\hline C & 1.585299 & 4.466242 & 2.604388 \\
\hline $\mathrm{C}$ & 1.632841 & 2.417139 & 3.877210 \\
\hline $\mathrm{H}$ & 1.706239 & 0.592864 & 2.731050 \\
\hline $\mathrm{H}$ & 1.593572 & 4.255089 & 0.459927 \\
\hline $\mathrm{H}$ & 1.580661 & 5.554452 & 2.567664 \\
\hline $\mathrm{H}$ & 1.667154 & 1.902920 & 4.836002 \\
\hline $\mathrm{H}$ & 1.595232 & 4.384344 & 4.754799 \\
\hline $\mathrm{H}$ & -4.670646 & 1.595135 & 0.634873 \\
\hline $\mathrm{H}$ & 3.705943 & 2.832832 & -1.829754 \\
\hline C & 2.670777 & 3.018679 & -2.097835 \\
\hline C & 1.533911 & 2.435159 & -1.447306 \\
\hline $\mathrm{H}$ & 2.852517 & 4.318319 & -3.905534 \\
\hline C & 0.356155 & 2.870143 & -2.172669 \\
\hline C & 0.806569 & 3.719771 & -3.238478 \\
\hline $\mathrm{H}$ & 0.206370 & 4.195471 & -4.011498 \\
\hline C & 2.223841 & 3.797123 & -3.191735 \\
\hline $\mathrm{Fe}$ & 1.608984 & 1.848586 & -3.380702 \\
\hline $\mathrm{H}$ & 1.649617 & -0.837785 & -2.546731 \\
\hline $\mathrm{C}$ & 1.746865 & -0.194660 & -3.417044 \\
\hline C & 2.945998 & 0.398492 & -3.905777 \\
\hline $\mathrm{H}$ & -0.368247 & -0.054707 & -4.115674 \\
\hline $\mathrm{H}$ & 3.931997 & 0.302749 & -3.461450 \\
\hline $\mathrm{C}$ & 2.608964 & 1.193301 & -5.037453 \\
\hline $\mathrm{H}$ & 3.295217 & 1.804081 & -5.614188 \\
\hline
\end{tabular}

\begin{tabular}{|c|c|c|c|}
\hline $\mathrm{C}$ & 1.204016 & 1.095920 & -5.244301 \\
\hline $\mathrm{H}$ & 0.646542 & 1.600346 & -6.027961 \\
\hline $\mathrm{C}$ & 0.672040 & 0.235096 & -4.239911 \\
\hline C & -1.069343 & 2.598884 & -1.757003 \\
\hline $\mathrm{H}$ & -1.278415 & 1.520255 & -1.793541 \\
\hline $\mathrm{N}$ & -2.031536 & 3.195237 & -2.810673 \\
\hline $\mathrm{C}$ & -3.445840 & 3.389848 & -2.357631 \\
\hline $\mathrm{H}$ & -3.472228 & 4.030933 & -1.475265 \\
\hline $\mathrm{H}$ & -3.873525 & 2.411239 & -2.125401 \\
\hline $\mathrm{H}$ & -3.996597 & 3.854647 & -3.179807 \\
\hline $\mathrm{C}$ & -2.037358 & 2.391320 & -4.073091 \\
\hline $\mathrm{H}$ & -1.007316 & 2.209656 & -4.386896 \\
\hline $\mathrm{H}$ & -2.591391 & 2.940261 & -4.839476 \\
\hline $\mathrm{H}$ & -2.545028 & 1.445073 & -3.854697 \\
\hline $\mathrm{Pd}$ & 0.099908 & -0.564706 & -0.052183 \\
\hline $\mathrm{C}$ & -0.873480 & -2.409417 & -0.318718 \\
\hline $\mathrm{C}$ & -1.784347 & -2.935975 & 0.506806 \\
\hline $\mathrm{H}$ & -1.895729 & -2.590916 & 1.535902 \\
\hline $\mathrm{C}$ & -0.642090 & -2.987410 & -1.691148 \\
\hline $\mathrm{H}$ & -1.050389 & -2.337081 & -2.482970 \\
\hline $\mathrm{H}$ & 0.432523 & -3.090256 & -1.902149 \\
\hline C & -1.303329 & -4.362119 & -1.757373 \\
\hline $\mathrm{H}$ & -0.729718 & -5.071526 & -1.131240 \\
\hline $\mathrm{H}$ & -1.328646 & -4.748245 & -2.781750 \\
\hline O & -2.636134 & -4.276637 & -1.311289 \\
\hline $\mathrm{C}$ & -2.695698 & -4.042905 & 0.066118 \\
\hline $\mathrm{H}$ & -3.735470 & -3.773438 & 0.305993 \\
\hline $\mathrm{H}$ & -2.466975 & -4.967073 & 0.636077 \\
\hline $\mathrm{O}$ & 1.004696 & -2.371987 & 2.080938 \\
\hline B & 1.028873 & -2.565778 & 0.731938 \\
\hline $\mathrm{O}$ & 1.537920 & -3.761340 & 0.321183 \\
\hline $\mathrm{C}$ & 2.152742 & -4.341072 & 1.512964 \\
\hline $\mathrm{C}$ & 1.343019 & -3.668681 & 2.665142 \\
\hline C & 2.121912 & -3.421788 & 3.937058 \\
\hline $\mathrm{H}$ & 2.981162 & -2.763858 & 3.769796 \\
\hline $\mathrm{H}$ & 2.483106 & -4.368581 & 4.360453 \\
\hline $\mathrm{H}$ & 1.469594 & -2.949487 & 4.682620 \\
\hline C & 0.038169 & -4.381752 & 2.974790 \\
\hline $\mathrm{H}$ & -0.518771 & -4.625395 & 2.060857 \\
\hline $\mathrm{H}$ & -0.588956 & -3.730155 & 3.598502 \\
\hline $\mathrm{H}$ & 0.215644 & -5.313909 & 3.524853 \\
\hline $\mathrm{C}$ & 3.613461 & -3.920632 & 1.483609 \\
\hline $\mathrm{H}$ & 3.723479 & -2.828432 & 1.543953 \\
\hline $\mathrm{H}$ & 4.059718 & -4.256781 & 0.539639 \\
\hline $\mathrm{H}$ & 4.180236 & -4.372613 & 2.306760 \\
\hline $\mathrm{C}$ & 2.027560 & -5.845639 & 1.437798 \\
\hline $\mathrm{H}$ & 0.990783 & -6.163910 & 1.284053 \\
\hline $\mathrm{H}$ & 2.403083 & -6.310015 & 2.359560 \\
\hline $\mathrm{H}$ & 2.626182 & -6.227484 & 0.602463 \\
\hline $\mathrm{H}$ & 1.470663 & -1.600562 & -0.061863 \\
\hline $\mathrm{H}$ & -1.656003 & 4.125896 & -3.029748 \\
\hline
\end{tabular}

SCF energy: -3329.866109680

M06-D3 (Gas Phase)/6-31G(d)+SDD (Pd, Fe) opt. 1 imaginary frequency: $\quad 201 \mathrm{i} \mathrm{cm-1}$ 


\begin{tabular}{|c|c|c|c|}
\hline \multicolumn{4}{|c|}{ TS-2RTania_conf1 } \\
\hline $\mathrm{P}$ & 0.290432 & -1.705745 & 0.930992 \\
\hline $\mathrm{P}$ & 1.212627 & 1.764179 & 0.231448 \\
\hline C & -1.025198 & -2.955630 & 1.245920 \\
\hline $\mathrm{C}$ & -3.020460 & -4.861663 & 1.752686 \\
\hline $\mathrm{C}$ & -2.004385 & -3.241545 & 0.286839 \\
\hline C & -1.063186 & -3.641037 & 2.466554 \\
\hline C & -2.056416 & -4.584303 & 2.715480 \\
\hline $\mathrm{C}$ & -2.990292 & -4.189402 & 0.533106 \\
\hline $\mathrm{H}$ & -2.021341 & -2.717330 & -0.665787 \\
\hline $\mathrm{H}$ & -0.321224 & -3.438816 & 3.237337 \\
\hline $\mathrm{H}$ & -2.074634 & -5.101615 & 3.672410 \\
\hline $\mathrm{H}$ & -3.744179 & -4.389904 & -0.226460 \\
\hline $\mathrm{H}$ & -3.797648 & -5.596086 & 1.953198 \\
\hline C & 1.993421 & 2.692229 & -1.139325 \\
\hline $\mathrm{C}$ & 3.138866 & 4.157005 & -3.227995 \\
\hline $\mathrm{C}$ & 3.135477 & 3.475172 & -0.913587 \\
\hline $\mathrm{C}$ & 1.445705 & 2.637366 & -2.425068 \\
\hline $\mathrm{C}$ & 2.018472 & 3.366106 & -3.465045 \\
\hline C & 3.697159 & 4.211247 & -1.951082 \\
\hline $\mathrm{H}$ & 0.551115 & 2.040704 & -2.605913 \\
\hline $\mathrm{H}$ & 1.570290 & 3.331818 & -4.456349 \\
\hline $\mathrm{H}$ & 4.574468 & 4.827655 & -1.764301 \\
\hline $\mathrm{H}$ & 3.577326 & 4.737624 & -4.037037 \\
\hline C & 0.531635 & 2.996423 & 1.393027 \\
\hline C & -0.621538 & 4.800893 & 3.188885 \\
\hline C & 1.072226 & 4.278057 & 1.551943 \\
\hline $\mathrm{C}$ & -0.606535 & 2.639545 & 2.125991 \\
\hline $\mathrm{C}$ & -1.175778 & 3.535322 & 3.023025 \\
\hline $\mathrm{C}$ & 0.497317 & 5.172135 & 2.447698 \\
\hline $\mathrm{H}$ & 1.932945 & 4.597015 & 0.967831 \\
\hline $\mathrm{H}$ & -1.085172 & 1.674799 & 1.957147 \\
\hline $\mathrm{H}$ & -2.065303 & 3.247666 & 3.580392 \\
\hline $\mathrm{H}$ & 0.922082 & 6.167251 & 2.560854 \\
\hline $\mathrm{H}$ & -1.070111 & 5.506372 & 3.885247 \\
\hline C & 2.672097 & 1.010836 & 1.079553 \\
\hline C & 4.837415 & -0.125259 & 2.466548 \\
\hline C & 3.040563 & 1.473215 & 2.347247 \\
\hline C & 3.398078 & -0.055866 & 0.513998 \\
\hline $\mathrm{C}$ & 4.457425 & -0.622239 & 1. 226848 \\
\hline C & 4.121637 & 0.925494 & 3.028612 \\
\hline $\mathrm{H}$ & 2.469461 & 2. 274619 & 2.813773 \\
\hline $\mathrm{H}$ & 4.989608 & -1.482772 & 0.813862 \\
\hline $\mathrm{H}$ & 4.391549 & 1.311235 & 4.009360 \\
\hline $\mathrm{H}$ & 5.673434 & -0.572135 & 3.000194 \\
\hline C & 0.934125 & -1.396444 & 2.605317 \\
\hline C & 1.689098 & -0.981537 & 5.256099 \\
\hline C & 0.180852 & -0.553506 & 3.429671 \\
\hline $\mathrm{C}$ & 2.056488 & -2.046189 & 3.120742 \\
\hline $\mathrm{C}$ & 2.429435 & -1.837563 & 4.446158 \\
\hline $\mathrm{C}$ & 0.561031 & -0.339597 & 4.747981 \\
\hline $\mathrm{H}$ & -0.724539 & -0.084253 & 3.040173 \\
\hline $\mathrm{H}$ & 2.632806 & -2.728089 & 2.494161 \\
\hline $\mathrm{H}$ & 3.300833 & -2.351353 & 4.848583 \\
\hline $\mathrm{H}$ & -0.028487 & 0.319226 & 5.382784 \\
\hline $\mathrm{H}$ & 1.983646 & -0.823785 & 6.291690 \\
\hline $\mathrm{H}$ & 3.605164 & 3.492648 & 0.071381 \\
\hline $\mathrm{H}$ & 0.948095 & -4.729914 & 0.474363 \\
\hline C & 1.668821 & -4.055319 & 0.021513 \\
\hline C & 1.585156 & -2.626458 & 0.053863 \\
\hline $\mathrm{H}$ & 3.093079 & -5.438562 & -1.005268 \\
\hline C & 2.684632 & -2.107336 & -0.733038 \\
\hline $\mathrm{C}$ & 3.427959 & -3.239173 & -1.209531 \\
\hline $\mathrm{H}$ & 4.296489 & -3.238758 & -1.865379 \\
\hline $\mathrm{C}$ & 2.793816 & -4.426742 & -0.753749 \\
\hline $\mathrm{Fe}$ & 1.498326 & -3.290724 & -1.863842 \\
\hline $\mathrm{H}$ & -1.017458 & -2.065378 & -2.260436 \\
\hline C & -0.284184 & -2.725318 & -2.713532 \\
\hline $\mathrm{C}$ & -0.215992 & -4.141255 & -2.583259 \\
\hline $\mathrm{H}$ & 1.006256 & -1.268949 & -3.801573 \\
\hline $\mathrm{H}$ & -0.877077 & -4.760833 & -1.985302 \\
\hline C & 0.911869 & -4.592141 & -3.324722 \\
\hline $\mathrm{H}$ & 1.258958 & -5.617334 & -3.396671 \\
\hline
\end{tabular}

\begin{tabular}{|c|c|c|c|}
\hline $\mathrm{C}$ & 1.542116 & -3.455832 & -3.906138 \\
\hline $\mathrm{H}$ & 2.436504 & -3.479843 & -4.521821 \\
\hline $\mathrm{C}$ & 0.799883 & -2.299691 & -3.524783 \\
\hline C & 3.055937 & -0.645904 & -0.836611 \\
\hline $\mathrm{H}$ & 2.246857 & -0.075248 & -1.316698 \\
\hline $\mathrm{N}$ & 4.249147 & -0.492207 & -1.806439 \\
\hline $\mathrm{C}$ & 4.995544 & 0.801731 & -1.712443 \\
\hline $\mathrm{H}$ & 5.333003 & 0.965620 & -0.687851 \\
\hline $\mathrm{H}$ & 4.327699 & 1.608922 & -2.023035 \\
\hline $\mathrm{H}$ & 5.852264 & 0.741927 & -2.389090 \\
\hline $\mathrm{C}$ & 3.840319 & -0.705843 & -3.229440 \\
\hline $\mathrm{H}$ & 3.283493 & -1.641484 & -3.305942 \\
\hline $\mathrm{H}$ & 4.736748 & -0.728651 & -3.855091 \\
\hline $\mathrm{H}$ & 3.207789 & 0.140736 & -3.518429 \\
\hline $\mathrm{Pd}$ & -0.523235 & 0.225851 & -0.273142 \\
\hline $\mathrm{C}$ & -1.645796 & 1.598774 & -1.441671 \\
\hline $\mathrm{C}$ & -1.902531 & 1.194946 & -2.697752 \\
\hline $\mathrm{H}$ & -1.853252 & 0.143211 & -2.991407 \\
\hline $\mathrm{C}$ & -1.835681 & 3.037412 & -1.052669 \\
\hline $\mathrm{H}$ & -2.375150 & 3.112497 & -0.095697 \\
\hline $\mathrm{H}$ & -0.861312 & 3.524690 & -0.888001 \\
\hline C & -2.592034 & 3.785235 & -2.147975 \\
\hline $\mathrm{H}$ & -2.515001 & 4.868776 & -2.009622 \\
\hline $\mathrm{H}$ & -3.664700 & 3.516289 & -2.133789 \\
\hline O & -2.043665 & 3.490496 & -3.412961 \\
\hline $\mathrm{C}$ & -2.302634 & 2.164834 & -3.771972 \\
\hline $\mathrm{H}$ & -3.374531 & 2.017135 & -4.017440 \\
\hline $\mathrm{H}$ & -1.737645 & 1.962028 & -4.693041 \\
\hline $\mathrm{O}$ & -3.137836 & 0.725399 & 0.838917 \\
\hline B & -2.857254 & 0.119291 & -0.352902 \\
\hline $\mathrm{O}$ & -3.927172 & -0.422810 & -0.992639 \\
\hline C & -5.102320 & 0.055379 & -0.271074 \\
\hline $\mathrm{C}$ & -4.521370 & 0.365606 & 1.145205 \\
\hline C & -4.450640 & -0.848428 & 2.057610 \\
\hline $\mathrm{H}$ & -3.979388 & -1.708390 & 1.561492 \\
\hline $\mathrm{H}$ & -5.446225 & -1.150440 & 2.405138 \\
\hline $\mathrm{H}$ & -3.846982 & -0.595796 & 2.938920 \\
\hline C & -5.170385 & 1.530955 & 1.857294 \\
\hline $\mathrm{H}$ & -5.057425 & 2.466649 & 1.298473 \\
\hline $\mathrm{H}$ & -4.711828 & 1.665584 & 2.844729 \\
\hline $\mathrm{H}$ & -6.241493 & 1.343486 & 2.011021 \\
\hline $\mathrm{C}$ & -6.150750 & -1.033046 & -0.298754 \\
\hline $\mathrm{H}$ & -5.753414 & -1.985522 & 0.069663 \\
\hline $\mathrm{H}$ & -6.503736 & -1.184882 & -1.325567 \\
\hline $\mathrm{H}$ & -7.016280 & -0.752271 & 0.316290 \\
\hline $\mathrm{C}$ & -5.584601 & 1.291966 & -1.008277 \\
\hline $\mathrm{H}$ & -4.834386 & 2.094261 & -0.978261 \\
\hline $\mathrm{H}$ & -6.519886 & 1.678473 & -0.585470 \\
\hline $\mathrm{H}$ & -5.765622 & 1.033363 & -2.058767 \\
\hline $\mathrm{H}$ & -1.877628 & -0.743309 & -0.578519 \\
\hline $\mathrm{H}$ & 4.907856 & -1.237919 & -1.551808 \\
\hline
\end{tabular}

SCF energy: -3329.862501350

M06-D3 (Gas Phase)/6-31G(d)+SDD (Pd, Fe) opt. 1 imaginary frequency: $\quad 218 \mathrm{i} \mathrm{cm-1}$ 


\begin{tabular}{|c|c|c|c|}
\hline \multicolumn{4}{|c|}{ TS-2STania_conf5 } \\
\hline $\mathrm{P}$ & -0.759028 & 0.029214 & 1.870772 \\
\hline $\mathrm{C}$ & 4.632814 & 0.123135 & 1.422587 \\
\hline $\mathrm{C}$ & 7.291296 & -0.758854 & 1.535370 \\
\hline $\mathrm{C}$ & 5.019094 & -1.065322 & 0.793298 \\
\hline $\mathrm{C}$ & 5.600561 & 0.862614 & 2.114053 \\
\hline $\mathrm{C}$ & 6.919470 & 0.423129 & 2.168096 \\
\hline $\mathrm{C}$ & 6.338110 & -1.502509 & 0.844770 \\
\hline $\mathrm{H}$ & 4.290694 & -1.677483 & 0.264293 \\
\hline $\mathrm{H}$ & 5.325809 & 1.782960 & 2.628401 \\
\hline $\mathrm{H}$ & 7.657100 & 1.005957 & 2.715760 \\
\hline $\mathrm{H}$ & 6.615544 & -2.434230 & 0.355199 \\
\hline $\mathrm{H}$ & 8.321636 & -1.104491 & 1.586206 \\
\hline $\mathrm{C}$ & -2.065899 & -0.057531 & 0.591410 \\
\hline C & -4.059226 & -0.192032 & -1.366312 \\
\hline $\mathrm{C}$ & -3.134957 & 0.852863 & 0.606228 \\
\hline C & -2.011739 & -1.034608 & -0.406331 \\
\hline C & -3.009433 & -1.106702 & -1.376892 \\
\hline C & -4.121906 & 0.788389 & -0.375287 \\
\hline $\mathrm{H}$ & -1.180662 & -1.739850 & -0.419205 \\
\hline $\mathrm{H}$ & -2.965222 & -1.880075 & -2.141879 \\
\hline $\mathrm{H}$ & -4.954032 & 1.489718 & -0.353484 \\
\hline $\mathrm{H}$ & -4.838351 & -0.248349 & -2.123702 \\
\hline C & -1.504469 & -0.638978 & 3.385904 \\
\hline $\mathrm{C}$ & -2.458243 & -1.916467 & 5.673512 \\
\hline C & -2.765526 & -1.240807 & 3.377173 \\
\hline $\mathrm{C}$ & -0.716972 & -0.689285 & 4.544906 \\
\hline C & -1.198321 & -1.319280 & 5.685340 \\
\hline C & -3.238664 & -1.876297 & 4.522249 \\
\hline $\mathrm{H}$ & -3.377941 & -1.232585 & 2.476167 \\
\hline $\mathrm{H}$ & 0.279012 & -0.242766 & 4.552678 \\
\hline $\mathrm{H}$ & -0.585206 & -1.351870 & 6.583793 \\
\hline $\mathrm{H}$ & -4.221437 & -2.342955 & 4.511939 \\
\hline $\mathrm{H}$ & -2.830939 & -2.416279 & 6.565103 \\
\hline C & -0.577877 & 1.843523 & 2.132402 \\
\hline $\mathrm{C}$ & -0.073187 & 4.556833 & 2.632776 \\
\hline C & -0.906893 & 2.417968 & 3.362355 \\
\hline $\mathrm{C}$ & -0.009742 & 2.655404 & 1.129514 \\
\hline $\mathrm{C}$ & 0.262895 & 3.996153 & 1.406031 \\
\hline $\mathrm{C}$ & -0.669932 & 3.767187 & 3.607506 \\
\hline $\mathrm{H}$ & -1.347561 & 1.803931 & 4.145580 \\
\hline $\mathrm{H}$ & 0.767137 & 4.606437 & 0.654922 \\
\hline $\mathrm{H}$ & -0.936557 & 4.193713 & 4.572038 \\
\hline $\mathrm{H}$ & 0.143422 & 5.604791 & 2.828923 \\
\hline $\mathrm{C}$ & 2.793150 & 1.661681 & 2.946640 \\
\hline $\mathrm{C}$ & 2.784060 & 2.933650 & 5.427235 \\
\hline $\mathrm{C}$ & 2.627330 & 0.913685 & 4.119749 \\
\hline C & 2.970147 & 3.043248 & 3.023177 \\
\hline $\mathrm{C}$ & 2.962796 & 3.676061 & 4.264148 \\
\hline C & 2.620510 & 1.551154 & 5.354592 \\
\hline $\mathrm{H}$ & 2.525812 & -0.174833 & 4.064194 \\
\hline $\mathrm{H}$ & 3.129464 & 3.626413 & 2.114708 \\
\hline $\mathrm{H}$ & 3.106382 & 4.753764 & 4.321661 \\
\hline $\mathrm{H}$ & 2.498235 & 0.965959 & 6.265099 \\
\hline $\mathrm{H}$ & 2.785112 & 3.431495 & 6.394806 \\
\hline $\mathrm{H}$ & -3.203804 & 1.601019 & 1.398746 \\
\hline $\mathrm{H}$ & 5.044744 & 2.241671 & -0.332414 \\
\hline C & 4.019305 & 2.463833 & -0.611775 \\
\hline $\mathrm{C}$ & 2.856041 & 1.913508 & 0.017409 \\
\hline $\mathrm{H}$ & 4.257220 & 3.777071 & -2.403749 \\
\hline $\mathrm{C}$ & 1.699285 & 2.402310 & -0.711835 \\
\hline C & 2.184267 & 3.242803 & -1.762912 \\
\hline $\mathrm{H}$ & 1.602216 & 3.745258 & -2.529394 \\
\hline C & 3.605098 & 3.268378 & -1.701864 \\
\hline $\mathrm{Fe}$ & 2.934088 & 1.347987 & -1.924299 \\
\hline $\mathrm{H}$ & 2.889636 & -1.380134 & -1.186600 \\
\hline C & 3.018143 & -0.701553 & -2.025243 \\
\hline $\mathrm{C}$ & 4.236877 & -0.122837 & -2.481214 \\
\hline $\mathrm{H}$ & 0.920806 & -0.496883 & -2.742525 \\
\hline $\mathrm{H}$ & 5.216308 & -0.259616 & -2.033211 \\
\hline C & 3.931329 & 0.717166 & -3.589355 \\
\hline $\mathrm{H}$ & 4.638994 & 1.330096 & -4.137327 \\
\hline C & 2.526072 & 0.665250 & -3.811568 \\
\hline
\end{tabular}

\begin{tabular}{|c|c|c|c|}
\hline $\mathrm{H}$ & 1.987837 & 1.211834 & -4.579990 \\
\hline $\mathrm{C}$ & 1.964919 & -0.215946 & -2.842558 \\
\hline $\mathrm{C}$ & 0.311445 & 2.088672 & -0.235888 \\
\hline $\mathrm{H}$ & 0.235796 & 0.991448 & -0.195299 \\
\hline $\mathrm{N}$ & -0.841846 & 2.451670 & -1.199006 \\
\hline $\mathrm{C}$ & -0.785627 & 1.719186 & -2.498196 \\
\hline $\mathrm{H}$ & -0.781060 & 0.647733 & -2.283873 \\
\hline $\mathrm{H}$ & 0.121480 & 2.002305 & -3.036870 \\
\hline $\mathrm{H}$ & -1.678705 & 1.972278 & -3.076388 \\
\hline $\mathrm{C}$ & -1.118230 & 3.905178 & -1.412058 \\
\hline $\mathrm{H}$ & -1.311507 & 4.382230 & -0.450238 \\
\hline $\mathrm{H}$ & -2.002715 & 3.988467 & -2.049165 \\
\hline $\mathrm{H}$ & -0.264414 & 4.375806 & -1.902317 \\
\hline $\mathrm{Pd}$ & 1.282335 & -1.096570 & 1.407130 \\
\hline $\mathrm{H}$ & -1.668906 & 2.073981 & -0.705611 \\
\hline $\mathrm{P}$ & 2.892425 & 0.720125 & 1.389376 \\
\hline $\mathrm{C}$ & 0.371214 & -3.006147 & 1.428871 \\
\hline $\mathrm{C}$ & -0.386038 & -3.504774 & 2.415532 \\
\hline $\mathrm{H}$ & -0.448318 & -3.021886 & 3.392852 \\
\hline $\mathrm{C}$ & 0.462484 & -3.713544 & 0.100533 \\
\hline $\mathrm{H}$ & 0.463880 & -3.007670 & -0.747657 \\
\hline $\mathrm{H}$ & 1.418276 & -4.258196 & 0.042176 \\
\hline $\mathrm{C}$ & -0.702378 & -4.690188 & -0.036700 \\
\hline $\mathrm{H}$ & -0.560473 & -5.361384 & -0.890402 \\
\hline $\mathrm{H}$ & -1.651718 & -4.136736 & -0.188949 \\
\hline O & -0.795629 & -5.499604 & 1.107328 \\
\hline C & -1.167214 & -4.773766 & 2.240963 \\
\hline $\mathrm{H}$ & -2.246697 & -4.508941 & 2.213526 \\
\hline $\mathrm{H}$ & -1.030065 & -5.437789 & 3.105251 \\
\hline O & 2.318698 & -2.538671 & 3.616087 \\
\hline B & 2.296000 & -2.947864 & 2.307616 \\
\hline O & 2.961138 & -4.105119 & 2.054619 \\
\hline $\mathrm{C}$ & 3.262428 & -4.678154 & 3.364359 \\
\hline C & 3.282642 & -3.412833 & 4.280127 \\
\hline C & 4.613874 & -2.678155 & 4.269786 \\
\hline $\mathrm{H}$ & 4.968785 & -2.488265 & 3.247082 \\
\hline $\mathrm{H}$ & 5.387860 & -3.240920 & 4.806051 \\
\hline $\mathrm{H}$ & 4.495974 & -1.708034 & 4.770970 \\
\hline $\mathrm{C}$ & 2.809159 & -3.638697 & 5.698294 \\
\hline $\mathrm{H}$ & 1.772937 & -3.993684 & 5.729331 \\
\hline $\mathrm{H}$ & 2.862881 & -2.695822 & 6.258570 \\
\hline $\mathrm{H}$ & 3.446885 & -4.369700 & 6.212922 \\
\hline $\mathrm{C}$ & 4.580368 & -5.411146 & 3.265475 \\
\hline $\mathrm{H}$ & 5.369405 & -4.772545 & 2.853408 \\
\hline $\mathrm{H}$ & 4.472199 & -6.284195 & 2.611280 \\
\hline $\mathrm{H}$ & 4.897830 & -5.768966 & 4.254236 \\
\hline $\mathrm{C}$ & 2.134360 & -5.641900 & 3.690146 \\
\hline $\mathrm{H}$ & 1.181563 & -5.117583 & 3.836507 \\
\hline $\mathrm{H}$ & 2.350620 & -6.216889 & 4.599010 \\
\hline $\mathrm{H}$ & 2.011553 & -6.345022 & 2.857272 \\
\hline $\mathrm{H}$ & 2.634548 & -2.124311 & 1.283763 \\
\hline
\end{tabular}

SCF energy: -3329.865506890

M06-D3 (Gas Phase)/6-31G(d)+SDD (Pd, Fe) opt. 1 imaginary frequency: $206 \mathrm{i} \mathrm{cm-1}$ 


\begin{tabular}{|c|c|c|c|}
\hline \multicolumn{4}{|c|}{ TS-2STania_confl } \\
\hline $\mathrm{P}$ & 1.629388 & 1.112090 & -0.114022 \\
\hline $\mathrm{P}$ & -1.990173 & 0.481346 & 0.519563 \\
\hline $\mathrm{C}$ & 3.344458 & 0.462049 & -0.252402 \\
\hline $\mathrm{C}$ & 5.965184 & -0.514875 & -0.418926 \\
\hline C & 3.645038 & -0.642548 & -1.057176 \\
\hline C & 4.377938 & 1.070747 & 0.470113 \\
\hline C & 5.678638 & 0.583198 & 0.385748 \\
\hline C & 4.945522 & -1.126458 & -1.144207 \\
\hline $\mathrm{H}$ & 2.861924 & -1.148175 & -1.620562 \\
\hline $\mathrm{H}$ & 4.170347 & 1.925099 & 1.113667 \\
\hline $\mathrm{H}$ & 6.469548 & 1.062194 & 0.959080 \\
\hline $\mathrm{H}$ & 5.157612 & -1.991811 & -1.769482 \\
\hline $\mathrm{H}$ & 6.981601 & -0.898576 & -0.476752 \\
\hline C & -3.407708 & 0.420559 & -0.628541 \\
\hline $\mathrm{C}$ & -5.545897 & 0.334515 & -2.424430 \\
\hline C & -4.638813 & 0.997170 & -0.287457 \\
\hline C & -3.259443 & -0.194479 & -1.874255 \\
\hline C & -4.323684 & -0.234413 & -2.773251 \\
\hline $\mathrm{C}$ & -5.704423 & 0.946155 & -1.180485 \\
\hline $\mathrm{H}$ & -2.301579 & -0.649099 & -2.133922 \\
\hline $\mathrm{H}$ & -4.203422 & -0.726729 & -3.736992 \\
\hline $\mathrm{H}$ & -6.662768 & 1.382541 & -0.905980 \\
\hline $\mathrm{H}$ & -6.382837 & 0.293441 & -3.118504 \\
\hline $\mathrm{C}$ & -2.562027 & -0.292341 & 2.066466 \\
\hline C & -3.207435 & -1.731713 & 4.372933 \\
\hline $\mathrm{C}$ & -3.782750 & -0.967553 & 2.156203 \\
\hline C & -1.658900 & -0.360257 & 3.136145 \\
\hline C & -1.983629 & -1.070285 & 4.284640 \\
\hline C & -4.102660 & -1.679609 & 3.309321 \\
\hline $\mathrm{H}$ & -4.482117 & -0.964612 & 1.321685 \\
\hline $\mathrm{H}$ & -0.691391 & 0.137525 & 3.066292 \\
\hline $\mathrm{H}$ & -1.275429 & -1.113061 & 5.110055 \\
\hline $\mathrm{H}$ & -5.056181 & -2.200077 & 3.371571 \\
\hline $\mathrm{H}$ & -3.460557 & -2.291974 & 5.270543 \\
\hline $\mathrm{C}$ & -1.784851 & 2.283835 & 0.892784 \\
\hline $\mathrm{C}$ & -1.261594 & 4.959056 & 1.596533 \\
\hline $\mathrm{C}$ & -2.049150 & 2.759309 & 2.181739 \\
\hline $\mathrm{C}$ & -1.266244 & 3.185188 & -0.058978 \\
\hline $\mathrm{C}$ & -0.988796 & 4.501673 & 0.313706 \\
\hline $\mathrm{C}$ & -1.804964 & 4.083863 & 2.529271 \\
\hline $\mathrm{H}$ & -2.451206 & 2.082340 & 2.932906 \\
\hline $\mathrm{H}$ & -0.540769 & 5.184056 & -0.413042 \\
\hline $\mathrm{H}$ & -2.028445 & 4.425053 & 3.537686 \\
\hline $\mathrm{H}$ & -1.044440 & 5.990235 & 1.866603 \\
\hline $\mathrm{C}$ & 1.663922 & 1.933351 & 1.510853 \\
\hline $\mathrm{C}$ & 1.821029 & 3.030146 & 4.067877 \\
\hline $\mathrm{C}$ & 1.608254 & 1.101437 & 2.636642 \\
\hline $\mathrm{C}$ & 1.819778 & 3.310210 & 1.670644 \\
\hline $\mathrm{C}$ & 1.894984 & 3.855422 & 2.950262 \\
\hline C & 1.683474 & 1.651796 & 3.910468 \\
\hline $\mathrm{H}$ & 1.518229 & 0.018380 & 2.507819 \\
\hline $\mathrm{H}$ & 1.895817 & 3.959429 & 0.797273 \\
\hline $\mathrm{H}$ & 2.020562 & 4.929908 & 3.073004 \\
\hline $\mathrm{H}$ & 1.644011 & 1.002125 & 4.783921 \\
\hline $\mathrm{H}$ & 1.885255 & 3.459742 & 5.065539 \\
\hline $\mathrm{H}$ & -4.764091 & 1.485887 & 0.680609 \\
\hline $\mathrm{H}$ & 3.728708 & 2.699084 & -1.806215 \\
\hline $\mathrm{C}$ & 2.702201 & 2.997859 & -1.995305 \\
\hline $\mathrm{C}$ & 1.551245 & 2.427040 & -1.361929 \\
\hline $\mathrm{H}$ & 2.905988 & 4.504524 & -3.632878 \\
\hline $\mathrm{C}$ & 0.381218 & 3.036951 & -1.958677 \\
\hline $\mathrm{C}$ & 0.850226 & 3.982058 & -2.932117 \\
\hline $\mathrm{H}$ & 0.256678 & 4.600316 & -3.602503 \\
\hline $\mathrm{C}$ & 2.270521 & 3.943402 & -2.956271 \\
\hline $\mathrm{Fe}$ & 1.485599 & 2.090209 & -3.357724 \\
\hline $\mathrm{H}$ & 1.266257 & -0.684714 & -2.902667 \\
\hline $\mathrm{C}$ & 1.409595 & 0.065500 & -3.676544 \\
\hline $\mathrm{C}$ & 2.649681 & 0.600632 & -4.130150 \\
\hline $\mathrm{H}$ & -0.696694 & 0.507459 & -4.264878 \\
\hline $\mathrm{H}$ & 3.633678 & 0.347742 & -3.747464 \\
\hline $\mathrm{C}$ & 2.365776 & 1.574892 & -5.128095 \\
\hline $\mathrm{H}$ & 3.095495 & 2.189981 & -5.643623 \\
\hline
\end{tabular}

\begin{tabular}{|c|c|c|c|}
\hline C & 0.953422 & 1.646613 & -5.287429 \\
\hline $\mathrm{H}$ & 0.428836 & 2.309573 & -5.969057 \\
\hline C & 0.363918 & 0.709892 & -4.388887 \\
\hline C & -1.037655 & 2.801000 & -1.501878 \\
\hline $\mathrm{H}$ & -1.325231 & 1.754925 & -1.674945 \\
\hline $\mathrm{N}$ & -2.004185 & 3.606155 & -2.406236 \\
\hline $\mathrm{C}$ & -3.377552 & 3.826471 & -1.849935 \\
\hline $\mathrm{H}$ & -3.316980 & 4.348523 & -0.894000 \\
\hline $\mathrm{H}$ & -3.860002 & 2.853969 & -1.719818 \\
\hline $\mathrm{H}$ & -3.935701 & 4.427034 & -2.573264 \\
\hline $\mathrm{C}$ & -2.128745 & 3.002017 & -3.769498 \\
\hline $\mathrm{H}$ & -1.131426 & 2.800970 & -4.165695 \\
\hline $\mathrm{H}$ & -2.675555 & 3.695360 & -4.414424 \\
\hline $\mathrm{H}$ & -2.694591 & 2.069481 & -3.662264 \\
\hline $\mathrm{Pd}$ & -0.021502 & -0.643253 & -0.177609 \\
\hline $\mathrm{C}$ & -0.996396 & -2.519517 & -0.247776 \\
\hline C & -1.667794 & -3.085049 & 0.764598 \\
\hline $\mathrm{H}$ & -1.603560 & -2.695755 & 1.782268 \\
\hline C & -1.067341 & -3.108490 & -1.632782 \\
\hline $\mathrm{H}$ & -1.095447 & -2.334285 & -2.416742 \\
\hline $\mathrm{H}$ & -0.160006 & -3.703945 & -1.823116 \\
\hline $\mathrm{C}$ & -2.303330 & -3.995813 & -1.735937 \\
\hline $\mathrm{H}$ & -2.301473 & -4.578890 & -2.662973 \\
\hline $\mathrm{H}$ & -3.219912 & -3.371699 & -1.720358 \\
\hline O & -2.325099 & -4.918105 & -0.675508 \\
\hline $\mathrm{C}$ & -2.523136 & -4.300828 & 0.560924 \\
\hline $\mathrm{H}$ & -3.584178 & -3.995325 & 0.691249 \\
\hline $\mathrm{H}$ & -2.319189 & -5.055917 & 1.332470 \\
\hline 0 & 1.139926 & -2.295028 & 1.797474 \\
\hline B & 0.993583 & -2.598614 & 0.467273 \\
\hline O & 1.593531 & -3.751830 & 0.071650 \\
\hline C & 1.973244 & -4.439680 & 1.302679 \\
\hline $\mathrm{C}$ & 2.110872 & -3.255905 & 2.313521 \\
\hline C & 3.467978 & -2.572276 & 2.261748 \\
\hline $\mathrm{H}$ & 3.755434 & -2.313595 & 1.233087 \\
\hline $\mathrm{H}$ & 4.253850 & -3.204064 & 2.693591 \\
\hline $\mathrm{H}$ & 3.427541 & -1.640950 & 2.842316 \\
\hline C & 1.731228 & -3.579961 & 3.741040 \\
\hline $\mathrm{H}$ & 0.686684 & -3.901889 & 3.821571 \\
\hline $\mathrm{H}$ & 1.859963 & -2.686229 & 4.366241 \\
\hline $\mathrm{H}$ & 2.375531 & -4.369824 & 4.149584 \\
\hline $\mathrm{C}$ & 3.251679 & -5.202180 & 1.040332 \\
\hline $\mathrm{H}$ & 4.029676 & -4.556692 & 0.618180 \\
\hline $\mathrm{H}$ & 3.060491 & -6.014603 & 0.329435 \\
\hline $\mathrm{H}$ & 3.631229 & -5.649771 & 1.968798 \\
\hline $\mathrm{C}$ & 0.840455 & -5.394035 & 1.640143 \\
\hline $\mathrm{H}$ & -0.077513 & -4.857223 & 1.909875 \\
\hline $\mathrm{H}$ & 1.108859 & -6.051958 & 2.475952 \\
\hline $\mathrm{H}$ & 0.624636 & -6.018458 & 0.764449 \\
\hline $\mathrm{H}$ & 1.274487 & -1.701487 & -0.507690 \\
\hline $\mathrm{H}$ & -1.571860 & 4.532304 & -2.509088 \\
\hline
\end{tabular}

SCF energy: -3329.866093840

M06-D3 (Gas Phase)/6-31G(d)+SDD (Pd, Fe) opt. 1 imaginary frequency: $\quad 207 \mathrm{i} \mathrm{cm-1}$ 


\begin{tabular}{|c|c|c|c|}
\hline \multicolumn{4}{|c|}{ Int-5STania_conf8 } \\
\hline $\mathrm{P}$ & 0.420693 & 2.168693 & 0.397253 \\
\hline $\mathrm{C}$ & -0.716213 & -3.243369 & 0.618308 \\
\hline $\mathrm{C}$ & -2.148411 & -5.642389 & 0.379977 \\
\hline $\mathrm{C}$ & -1.286488 & -3.618282 & -0.602676 \\
\hline $\mathrm{C}$ & -0.877906 & -4.089318 & 1.721496 \\
\hline $\mathrm{C}$ & -1.585763 & -5.281514 & 1.599578 \\
\hline $\mathrm{C}$ & -1.995661 & -4.807848 & -0.724348 \\
\hline $\mathrm{H}$ & -1.181772 & -2.980683 & -1.478083 \\
\hline $\mathrm{H}$ & -0.457250 & -3.817993 & 2.688944 \\
\hline $\mathrm{H}$ & -1.701913 & -5.927146 & 2.467660 \\
\hline $\mathrm{H}$ & -2.433289 & -5.080020 & -1.683388 \\
\hline $\mathrm{H}$ & -2.707348 & -6.571455 & 0.289490 \\
\hline $\mathrm{C}$ & 1.508233 & 3.251144 & -0.620634 \\
\hline C & 3.211621 & 4.892464 & -2.130676 \\
\hline $\mathrm{C}$ & 2.643226 & 3.840742 & -0.032047 \\
\hline C & 1.213651 & 3.543154 & -1.957190 \\
\hline C & 2.062449 & 4.356935 & -2.704383 \\
\hline $\mathrm{C}$ & 3.494497 & 4.644583 & -0.788404 \\
\hline $\mathrm{H}$ & 0.299030 & 3.167577 & -2.409143 \\
\hline $\mathrm{H}$ & 1.814227 & 4.577130 & -3.740603 \\
\hline $\mathrm{H}$ & 4.364908 & 5.099325 & -0.318314 \\
\hline $\mathrm{H}$ & 3.873149 & 5.524765 & -2.719209 \\
\hline C & -0.779014 & 3.281500 & 1.178049 \\
\hline $\mathrm{C}$ & -2.763220 & 4.898527 & 2.275231 \\
\hline C & -0.802574 & 4.649062 & 0.891606 \\
\hline $\mathrm{C}$ & -1.755522 & 2.724717 & 2.014159 \\
\hline C & -2.737310 & 3.535800 & 2.568117 \\
\hline C & -1.797669 & 5.452592 & 1.440416 \\
\hline $\mathrm{H}$ & -0.057439 & 5.090629 & 0.231111 \\
\hline $\mathrm{H}$ & -1.740711 & 1.654641 & 2.232197 \\
\hline $\mathrm{H}$ & -3.490035 & 3.103978 & 3.225436 \\
\hline $\mathrm{H}$ & -1.817137 & 6.516291 & 1.213365 \\
\hline $\mathrm{H}$ & -3.539730 & 5.530444 & 2.701057 \\
\hline C & 1.576044 & 1.643601 & 1.730382 \\
\hline $\mathrm{C}$ & 3.297619 & 0.838367 & 3.790955 \\
\hline C & 1.408974 & 2.125526 & 3.031420 \\
\hline $\mathrm{C}$ & 2.630783 & 0.748119 & 1.460989 \\
\hline $\mathrm{C}$ & 3.463587 & 0.339940 & 2.504207 \\
\hline $\mathrm{C}$ & 2.268582 & 1.734018 & 4.053013 \\
\hline $\mathrm{H}$ & 0.598399 & 2.816914 & 3.253691 \\
\hline $\mathrm{H}$ & 4.239429 & -0.402186 & 2.309457 \\
\hline $\mathrm{H}$ & 2.121198 & 2.122540 & 5.058338 \\
\hline $\mathrm{H}$ & 3.961142 & 0.512853 & 4.589323 \\
\hline C & 0.085623 & -1.364145 & 2.589335 \\
\hline $\mathrm{C}$ & -0.327242 & -0.968681 & 5.320607 \\
\hline $\mathrm{C}$ & -1.124076 & -0.829077 & 3.050573 \\
\hline C & 1.076528 & -1.718569 & 3.504543 \\
\hline $\mathrm{C}$ & 0.868271 & -1.517554 & 4.867532 \\
\hline C & -1.326753 & -0.629038 & 4.410250 \\
\hline $\mathrm{H}$ & -1.920392 & -0.607445 & 2.335341 \\
\hline $\mathrm{H}$ & 2.008870 & -2.166137 & 3.157255 \\
\hline $\mathrm{H}$ & 1.642333 & -1.800516 & 5.579132 \\
\hline $\mathrm{H}$ & -2.272571 & -0.219396 & 4.762967 \\
\hline $\mathrm{H}$ & -0.487172 & -0.818856 & 6.386470 \\
\hline $\mathrm{H}$ & 2.859247 & 3.681165 & 1.025923 \\
\hline $\mathrm{H}$ & 1.853548 & -4.365329 & 0.748241 \\
\hline C & 2.445180 & -3.481790 & 0.526981 \\
\hline $\mathrm{C}$ & 1.935413 & -2.146157 & 0.464546 \\
\hline $\mathrm{H}$ & 4.462824 & -4.326445 & 0.065974 \\
\hline $\mathrm{C}$ & 3.031813 & -1.287494 & 0.055343 \\
\hline C & 4.185535 & -2.115828 & -0.113215 \\
\hline $\mathrm{H}$ & 5.170653 & -1.814215 & -0.455948 \\
\hline C & 3.816284 & -3.461139 & 0.166585 \\
\hline $\mathrm{Fe}$ & 2.666466 & -2.707536 & -1.344660 \\
\hline $\mathrm{H}$ & 0.230202 & -2.116320 & -2.647136 \\
\hline $\mathrm{C}$ & 1.198585 & -2.590635 & -2.773306 \\
\hline $\mathrm{C}$ & 1.529851 & -3.948401 & -2.496783 \\
\hline $\mathrm{H}$ & 2.425880 & -0.900088 & -3.549013 \\
\hline $\mathrm{H}$ & 0.862025 & -4.700245 & -2.087685 \\
\hline C & 2.911297 & -4.126814 & -2.790234 \\
\hline $\mathrm{H}$ & 3.479118 & -5.039786 & -2.645554 \\
\hline C & 3.434646 & -2.881458 & -3.238305 \\
\hline
\end{tabular}

\begin{tabular}{|c|c|c|c|}
\hline $\mathrm{H}$ & 4.466368 & -2.691210 & -3.518019 \\
\hline $\mathrm{C}$ & 2.370691 & -1.935500 & -3.232399 \\
\hline C & 2.854107 & 0.199327 & 0.067837 \\
\hline $\mathrm{H}$ & 1.964041 & 0.413072 & -0.546355 \\
\hline $\mathrm{N}$ & 3.933116 & 1.037812 & -0.640576 \\
\hline $\mathrm{C}$ & 4.006499 & 0.786443 & -2.106302 \\
\hline $\mathrm{H}$ & 3.005346 & 0.931804 & -2.525048 \\
\hline $\mathrm{H}$ & 4.345575 & -0.237347 & -2.283345 \\
\hline $\mathrm{H}$ & 4.698744 & 1.510165 & -2.546820 \\
\hline C & 5.289498 & 1.089008 & -0.021166 \\
\hline $\mathrm{H}$ & 5.195089 & 1.375808 & 1.028347 \\
\hline $\mathrm{H}$ & 5.871395 & 1.844689 & -0.557537 \\
\hline $\mathrm{H}$ & 5.778817 & 0.117366 & -0.107229 \\
\hline $\mathrm{Pd}$ & -0.655653 & 0.301084 & -0.541050 \\
\hline $\mathrm{H}$ & 3.559723 & 1.998908 & -0.548436 \\
\hline $\mathrm{P}$ & 0.207804 & -1.659425 & 0.791046 \\
\hline $\mathrm{C}$ & -1.471220 & 1.624377 & -1.870489 \\
\hline $\mathrm{C}$ & -2.290915 & 2.637638 & -1.606205 \\
\hline $\mathrm{H}$ & -2.568581 & 2.926940 & -0.590753 \\
\hline $\mathrm{C}$ & -1.147773 & 1.184292 & -3.269662 \\
\hline $\mathrm{H}$ & -0.090735 & 1.383909 & -3.517709 \\
\hline $\mathrm{H}$ & -1.287986 & 0.098800 & -3.392227 \\
\hline $\mathrm{C}$ & -2.082629 & 1.911005 & -4.247009 \\
\hline $\mathrm{H}$ & -3.084793 & 1.447880 & -4.203857 \\
\hline $\mathrm{H}$ & -1.710769 & 1.837252 & -5.274639 \\
\hline 0 & -2.161674 & 3.275774 & -3.927054 \\
\hline $\mathrm{C}$ & -2.856585 & 3.473994 & -2.726323 \\
\hline $\mathrm{H}$ & -2.768543 & 4.540509 & -2.478657 \\
\hline $\mathrm{H}$ & -3.934904 & 3.253109 & -2.855210 \\
\hline O & -3.256358 & -0.906702 & 0.379939 \\
\hline B & -2.937288 & -0.778479 & -0.927486 \\
\hline O & -3.953991 & -0.598381 & -1.786658 \\
\hline C & -5.166954 & -0.859193 & -1.003324 \\
\hline $\mathrm{C}$ & -4.685759 & -0.598963 & 0.462172 \\
\hline C & -5.298424 & -1.500066 & 1.509977 \\
\hline $\mathrm{H}$ & -5.080621 & -2.555854 & 1.317921 \\
\hline $\mathrm{H}$ & -6.387669 & -1.365785 & 1.548403 \\
\hline $\mathrm{H}$ & -4.893558 & -1.246549 & 2.498471 \\
\hline C & -4.774710 & 0.855923 & 0.886968 \\
\hline $\mathrm{H}$ & -4.312871 & 1.524276 & 0.147091 \\
\hline $\mathrm{H}$ & -4.236635 & 0.977739 & 1.837471 \\
\hline $\mathrm{H}$ & -5.813869 & 1.170018 & 1.042107 \\
\hline $\mathrm{C}$ & -5.531145 & -2.309141 & -1.267250 \\
\hline $\mathrm{H}$ & -4.741237 & -2.993058 & -0.926384 \\
\hline $\mathrm{H}$ & -5.663802 & -2.452866 & -2.345785 \\
\hline $\mathrm{H}$ & -6.468661 & -2.584801 & -0.769433 \\
\hline $\mathrm{C}$ & -6.253536 & 0.071935 & -1.488651 \\
\hline $\mathrm{H}$ & -5.929457 & 1.118751 & -1.473624 \\
\hline $\mathrm{H}$ & -7.152922 & -0.027092 & -0.866058 \\
\hline $\mathrm{H}$ & -6.529080 & -0.183351 & -2.518409 \\
\hline $\mathrm{H}$ & -1.796541 & -0.967156 & -1.356609 \\
\hline
\end{tabular}

SCF energy: -3329.875191270

M06-D3 (Gas Phase) /6-31G(d)+SDD (Pd, Fe) opt. No imaginary frequency 


\begin{tabular}{|c|c|c|c|}
\hline \multicolumn{4}{|c|}{ TS-2RTania_conf4 } \\
\hline $\mathrm{P}$ & 0.536207 & 2.121852 & 0.245655 \\
\hline $\mathrm{C}$ & -0.100546 & -3.135382 & 1.194284 \\
\hline $\mathrm{C}$ & -1.335412 & -5.625764 & 1.568386 \\
\hline $\mathrm{C}$ & -0.905901 & -3.692381 & 0.194816 \\
\hline C & 0.071038 & -3.845108 & 2.389936 \\
\hline $\mathrm{C}$ & -0.542568 & -5.080338 & 2.572803 \\
\hline $\mathrm{C}$ & -1.514556 & -4.929218 & 0.376530 \\
\hline $\mathrm{H}$ & -1.090886 & -3.161489 & -0.736138 \\
\hline $\mathrm{H}$ & 0.678972 & -3.432360 & 3.193794 \\
\hline $\mathrm{H}$ & -0.403361 & -5.613983 & 3.510629 \\
\hline $\mathrm{H}$ & -2.141709 & -5.339123 & -0.413145 \\
\hline $\mathrm{H}$ & -1.819442 & -6.588746 & 1.716793 \\
\hline $\mathrm{C}$ & 1.231332 & 3.070301 & -1.165294 \\
\hline C & 2.312355 & 4.457818 & -3.345943 \\
\hline $\mathrm{C}$ & 2.287856 & 3.978560 & -0.977846 \\
\hline C & 0.727336 & 2.868879 & -2.454672 \\
\hline C & 1.260019 & 3.566577 & -3.536746 \\
\hline $\mathrm{C}$ & 2.827465 & 4.663071 & -2.066013 \\
\hline $\mathrm{H}$ & -0.085145 & 2.155975 & -2.607309 \\
\hline $\mathrm{H}$ & 0.851811 & 3.409848 & -4.533602 \\
\hline $\mathrm{H}$ & 3.638303 & 5.372586 & -1.910528 \\
\hline $\mathrm{H}$ & 2.727917 & 5.001092 & -4.192117 \\
\hline C & -0.558946 & 3.247127 & 1.157585 \\
\hline $\mathrm{C}$ & -2.276404 & 4.882585 & 2.617764 \\
\hline C & -0.395523 & 4.636085 & 1.128154 \\
\hline $\mathrm{C}$ & -1.593887 & 2.683000 & 1.912815 \\
\hline C & -2.446487 & 3.500586 & 2.643732 \\
\hline C & -1.255438 & 5.448994 & 1.858438 \\
\hline $\mathrm{H}$ & 0.393375 & 5.090476 & 0.529416 \\
\hline $\mathrm{H}$ & -1.759913 & 1.605353 & 1.890719 \\
\hline $\mathrm{H}$ & -3.255570 & 3.058447 & 3.221680 \\
\hline $\mathrm{H}$ & -1.130907 & 6.529326 & 1.830178 \\
\hline $\mathrm{H}$ & -2.949454 & 5.523533 & 3.183413 \\
\hline C & 2.013782 & 1.908377 & 1.322039 \\
\hline $\mathrm{C}$ & 4.184308 & 1.483095 & 3.042593 \\
\hline $\mathrm{C}$ & 2.050299 & 2.502670 & 2.585405 \\
\hline $\mathrm{C}$ & 3.084708 & 1.086750 & 0.918965 \\
\hline $\mathrm{C}$ & 4.144425 & 0.862137 & 1.799705 \\
\hline $\mathrm{C}$ & 3.135488 & 2.306404 & 3.433679 \\
\hline $\mathrm{H}$ & 1.218474 & 3.122389 & 2.916657 \\
\hline $\mathrm{H}$ & 4.939470 & 0.169407 & 1.519334 \\
\hline $\mathrm{H}$ & 3.147342 & 2.782895 & 4.411631 \\
\hline $\mathrm{H}$ & 5.022914 & 1.304233 & 3.712218 \\
\hline C & 0.955000 & -0.973530 & 2.712213 \\
\hline $\mathrm{C}$ & 1.069589 & -0.265279 & 5.404623 \\
\hline C & -0.161465 & -0.410824 & 3.341043 \\
\hline C & 2.121797 & -1.199369 & 3.443018 \\
\hline C & 2.174738 & -0.845144 & 4.788946 \\
\hline C & -0.102256 & -0.050626 & 4.681045 \\
\hline $\mathrm{H}$ & -1.088659 & -0.279398 & 2.777057 \\
\hline $\mathrm{H}$ & 2.985490 & -1.666406 & 2.967289 \\
\hline $\mathrm{H}$ & 3.083634 & -1.027713 & 5.359829 \\
\hline $\mathrm{H}$ & -0.972830 & 0.388186 & 5.165051 \\
\hline $\mathrm{H}$ & 1.115868 & 0.008158 & 6.456848 \\
\hline $\mathrm{H}$ & 2.688656 & 4.153517 & 0.022962 \\
\hline $\mathrm{H}$ & 2.467345 & -4.124056 & 0.779736 \\
\hline C & 2.954081 & -3.240606 & 0.377316 \\
\hline $\mathrm{C}$ & 2.376516 & -1.930505 & 0.333279 \\
\hline $\mathrm{H}$ & 4.873478 & -4.044950 & -0.436797 \\
\hline $\mathrm{C}$ & 3.320133 & -1.066428 & -0.353737 \\
\hline C & 4.452083 & -1.866414 & -0.704114 \\
\hline $\mathrm{H}$ & 5.329229 & -1.557356 & -1.264738 \\
\hline C & 4.217680 & -3.198441 & -0.263060 \\
\hline $\mathrm{Fe}$ & 2.739453 & -2.602541 & -1.543863 \\
\hline $\mathrm{H}$ & -0.001150 & -2.438054 & -2.225472 \\
\hline C & 0.971027 & -2.765684 & -2.578156 \\
\hline $\mathrm{C}$ & 1.560865 & -4.045934 & -2.382253 \\
\hline $\mathrm{H}$ & 1.703412 & -0.935409 & -3.611456 \\
\hline $\mathrm{H}$ & 1.129005 & -4.870277 & -1.823649 \\
\hline $\mathrm{C}$ & 2.844967 & -4.030730 & -2.996731 \\
\hline $\mathrm{H}$ & 3.562824 & -4.844017 & -2.991673 \\
\hline C & 3.050811 & -2.740310 & -3.562433 \\
\hline
\end{tabular}

\begin{tabular}{|c|c|c|c|}
\hline $\mathrm{H}$ & 3.943814 & -2.419227 & -4.090215 \\
\hline C & 1.887170 & -1.959864 & -3.302209 \\
\hline C & 3.069018 & 0.409493 & -0.434300 \\
\hline $\mathrm{H}$ & 2.070632 & 0.528449 & -0.882350 \\
\hline $\mathrm{N}$ & 3.948510 & 1.213178 & -1.413736 \\
\hline $\mathrm{C}$ & 3.797139 & 0.781932 & -2.833032 \\
\hline $\mathrm{H}$ & 2.737715 & 0.842065 & -3.095666 \\
\hline $\mathrm{H}$ & 4.155852 & -0.244420 & -2.939691 \\
\hline $\mathrm{H}$ & 4.371589 & 1.464834 & -3.465248 \\
\hline $\mathrm{C}$ & 5.386349 & 1.394808 & -1.053203 \\
\hline $\mathrm{H}$ & 5.455730 & 1.848193 & -0.063018 \\
\hline $\mathrm{H}$ & 5.835798 & 2.060399 & -1.795390 \\
\hline $\mathrm{H}$ & 5.897461 & 0.430439 & -1.065154 \\
\hline $\mathrm{Pd}$ & -0.576614 & 0.091815 & -0.308143 \\
\hline $\mathrm{H}$ & 3.526680 & 2.157221 & -1.363926 \\
\hline $\mathrm{P}$ & 0.731225 & -1.505986 & 0.985205 \\
\hline $\mathrm{C}$ & -2.052091 & 0.972919 & -1.578172 \\
\hline C & -2.170844 & 0.426885 & -2.804594 \\
\hline $\mathrm{H}$ & -1.648685 & -0.494042 & -3.077073 \\
\hline $\mathrm{C}$ & -2.790253 & 2.246395 & -1.259410 \\
\hline $\mathrm{H}$ & -3.596561 & 2.040333 & -0.538917 \\
\hline $\mathrm{H}$ & -2.137100 & 2.981514 & -0.767912 \\
\hline C & -3.345761 & 2.870394 & -2.535735 \\
\hline $\mathrm{H}$ & -2.526930 & 3.337286 & -3.118197 \\
\hline $\mathrm{H}$ & -4.079275 & 3.650132 & -2.305848 \\
\hline O & -4.003452 & 1.907061 & -3.322725 \\
\hline C & -3.107375 & 0.971730 & -3.841754 \\
\hline $\mathrm{H}$ & -3.703525 & 0.149279 & -4.261209 \\
\hline $\mathrm{H}$ & -2.519389 & 1.400978 & -4.680497 \\
\hline O & -3.329436 & -0.227653 & 0.539957 \\
\hline B & -2.746708 & -0.735102 & -0.589131 \\
\hline 0 & -3.488532 & -1.674438 & -1.246424 \\
\hline C & -4.816817 & -1.618169 & -0.642523 \\
\hline C & -4.509407 & -1.054991 & 0.780970 \\
\hline C & -4.082194 & -2.118914 & 1.780252 \\
\hline $\mathrm{H}$ & -3.286846 & -2.763374 & 1.378367 \\
\hline $\mathrm{H}$ & -4.924967 & -2.754150 & 2.079160 \\
\hline $\mathrm{H}$ & -3.694893 & -1.626146 & 2.681452 \\
\hline $\mathrm{C}$ & -5.594163 & -0.177636 & 1.363221 \\
\hline $\mathrm{H}$ & -5.792800 & 0.696349 & 0.733160 \\
\hline $\mathrm{H}$ & -5.294083 & 0.176647 & 2.357346 \\
\hline $\mathrm{H}$ & -6.528879 & -0.742578 & 1.478360 \\
\hline $\mathrm{C}$ & -5.402684 & -3.011914 & -0.650601 \\
\hline $\mathrm{H}$ & -4.735669 & -3.736951 & -0.171402 \\
\hline $\mathrm{H}$ & -5.576148 & -3.337273 & -1.683000 \\
\hline $\mathrm{H}$ & -6.368582 & -3.026619 & -0.127820 \\
\hline $\mathrm{C}$ & -5.645728 & -0.682213 & -1.505433 \\
\hline $\mathrm{H}$ & -5.213423 & 0.326340 & -1.566117 \\
\hline $\mathrm{H}$ & -6.675891 & -0.599824 & -1.138071 \\
\hline $\mathrm{H}$ & -5.686335 & -1.084294 & -2.525240 \\
\hline $\mathrm{H}$ & -1.507102 & -1.267688 & -0.646883 \\
\hline
\end{tabular}

SCF energy: -3329.861758790

M06-D3 (Gas Phase) /6-31G(d) +SDD (Pd, Fe) opt. 1 imaginary frequency: $\quad 187 \mathrm{i} \mathrm{cm-1}$ 


\begin{tabular}{|c|c|c|c|}
\hline \multicolumn{4}{|c|}{ TS-2STania_conf2 } \\
\hline $\mathrm{P}$ & 1.596658 & 1.100185 & -0.122394 \\
\hline $\mathrm{P}$ & -2.040543 & 0.532745 & 0.396488 \\
\hline $\mathrm{C}$ & 3.305708 & 0.421922 & -0.173254 \\
\hline $\mathrm{C}$ & 5.912524 & -0.604003 & -0.208022 \\
\hline $\mathrm{C}$ & 3.613080 & -0.721398 & -0.919410 \\
\hline $\mathrm{C}$ & 4.324680 & 1.044538 & 0.557358 \\
\hline $\mathrm{C}$ & 5.618918 & 0.532910 & 0.538113 \\
\hline $\mathrm{C}$ & 4.906727 & -1.230546 & -0.940301 \\
\hline $\mathrm{H}$ & 2.839463 & -1.237059 & -1.487366 \\
\hline $\mathrm{H}$ & 4.110138 & 1.928857 & 1.156728 \\
\hline $\mathrm{H}$ & 6.398814 & 1.023648 & 1.116584 \\
\hline $\mathrm{H}$ & 5.124299 & -2.125833 & -1.519992 \\
\hline $\mathrm{H}$ & 6.923424 & -1.006163 & -0.214750 \\
\hline C & -3.392216 & 0.434285 & -0.824321 \\
\hline $\mathrm{C}$ & -5.423275 & 0.307541 & -2.736829 \\
\hline C & -4.643994 & 1.007123 & -0.561723 \\
\hline C & -3.167621 & -0.193787 & -2.052055 \\
\hline $\mathrm{C}$ & -4.178606 & -0.254778 & -3.008962 \\
\hline $\mathrm{C}$ & -5.656431 & 0.935651 & -1.512891 \\
\hline $\mathrm{H}$ & -2.190861 & -0.639935 & -2.250690 \\
\hline $\mathrm{H}$ & -4.000857 & -0.759911 & -3.957002 \\
\hline $\mathrm{H}$ & -6.631841 & 1.368348 & -1.299529 \\
\hline $\mathrm{H}$ & -6.219769 & 0.248549 & -3.475730 \\
\hline $\mathrm{C}$ & -2.700169 & -0.155295 & 1.948967 \\
\hline C & -3.486078 & -1.460439 & 4.291812 \\
\hline $\mathrm{C}$ & -3.913701 & -0.847685 & 1.996009 \\
\hline C & -1.873082 & -0.140278 & 3.080775 \\
\hline C & -2.267961 & -0.784155 & 4.246337 \\
\hline C & -4.304880 & -1.490545 & 3.167768 \\
\hline $\mathrm{H}$ & -4.552385 & -0.909744 & 1.116406 \\
\hline $\mathrm{H}$ & -0.913986 & 0.378256 & 3.051463 \\
\hline $\mathrm{H}$ & -1.620044 & -0.760958 & 5.120557 \\
\hline $\mathrm{H}$ & -5.254223 & -2.021659 & 3.196937 \\
\hline $\mathrm{H}$ & -3.794512 & -1.966762 & 5.204040 \\
\hline $\mathrm{C}$ & -1.830438 & 2.350897 & 0.692552 \\
\hline C & -1.306815 & 5.052270 & 1.296653 \\
\hline $\mathrm{C}$ & -2.145851 & 2.889689 & 1.944668 \\
\hline $\mathrm{C}$ & -1.265263 & 3.204632 & -0.276532 \\
\hline $\mathrm{C}$ & -0.987257 & 4.533732 & 0.048514 \\
\hline C & -1.901668 & 4.226239 & 2.242487 \\
\hline $\mathrm{H}$ & -2.590729 & 2.254458 & 2.707538 \\
\hline $\mathrm{H}$ & -0.501974 & 5.177757 & -0.689280 \\
\hline $\mathrm{H}$ & -2.166081 & 4.615148 & 3.223331 \\
\hline $\mathrm{H}$ & -1.087929 & 6.092214 & 1.529044 \\
\hline $\mathrm{C}$ & 1.583913 & 1.999336 & 1.460886 \\
\hline $\mathrm{C}$ & 1.652640 & 3.215559 & 3.967371 \\
\hline C & 1.459429 & 1.224348 & 2.621194 \\
\hline $\mathrm{C}$ & 1.762371 & 3.379147 & 1.561848 \\
\hline C & 1.793253 & 3.984131 & 2.816146 \\
\hline $\mathrm{C}$ & 1.491694 & 1.834215 & 3.869586 \\
\hline $\mathrm{H}$ & 1.350212 & 0.138433 & 2.540037 \\
\hline $\mathrm{H}$ & 1.889976 & 3.983621 & 0.662691 \\
\hline $\mathrm{H}$ & 1.935915 & 5.060714 & 2.893128 \\
\hline $\mathrm{H}$ & 1.399411 & 1.228619 & 4.770361 \\
\hline $\mathrm{H}$ & 1.682225 & 3.691781 & 4.945314 \\
\hline $\mathrm{H}$ & -4.827151 & 1.508633 & 0.390327 \\
\hline $\mathrm{H}$ & 3.781800 & 2.564265 & -1.812327 \\
\hline C & 2.768916 & 2.872532 & -2.052777 \\
\hline C & 1.584783 & 2.354374 & -1.434991 \\
\hline $\mathrm{H}$ & 3.061730 & 4.293921 & -3.751747 \\
\hline C & 0.449723 & 2.956178 & -2.103494 \\
\hline C & 0.972165 & 3.844354 & -3.102734 \\
\hline $\mathrm{H}$ & 0.415018 & 4.441722 & -3.821374 \\
\hline C & 2.391210 & 3.777957 & -3.073055 \\
\hline $\mathrm{Fe}$ & 1.582621 & 1.921749 & -3.412988 \\
\hline $\mathrm{H}$ & 1.241767 & -0.812182 & -2.826692 \\
\hline $\mathrm{C}$ & 1.449371 & -0.111669 & -3.632555 \\
\hline C & 2.728227 & 0.357792 & -4.051317 \\
\hline $\mathrm{H}$ & -0.608940 & 0.367066 & -4.347684 \\
\hline $\mathrm{H}$ & 3.683168 & 0.094706 & -3.606871 \\
\hline $\mathrm{C}$ & 2.526857 & 1.286871 & -5.110492 \\
\hline $\mathrm{H}$ & 3.301708 & 1.850082 & -5.619391 \\
\hline
\end{tabular}

\begin{tabular}{|c|c|c|c|}
\hline $\mathrm{C}$ & 1.127228 & 1.396544 & -5.342718 \\
\hline $\mathrm{H}$ & 0.658806 & 2.040600 & -6.081041 \\
\hline $\mathrm{C}$ & 0.462656 & 0.528043 & -4.428431 \\
\hline C & -0.988944 & 2.760542 & -1.693687 \\
\hline $\mathrm{H}$ & -1.287027 & 1.714146 & -1.841670 \\
\hline $\mathrm{N}$ & -1.908937 & 3.546136 & -2.661356 \\
\hline $\mathrm{C}$ & -3.301393 & 3.793970 & -2.166052 \\
\hline $\mathrm{H}$ & -3.273718 & 4.351348 & -1.228757 \\
\hline $\mathrm{H}$ & -3.794608 & 2.829396 & -2.017953 \\
\hline $\mathrm{H}$ & -3.828018 & 4.369574 & -2.932035 \\
\hline $\mathrm{C}$ & -1.987271 & 2.902946 & -4.010431 \\
\hline $\mathrm{H}$ & -0.978648 & 2.673914 & -4.359741 \\
\hline $\mathrm{H}$ & -2.495612 & 3.585254 & -4.697259 \\
\hline $\mathrm{H}$ & -2.572771 & 1.983222 & -3.899398 \\
\hline $\mathrm{Pd}$ & -0.064114 & -0.654177 & -0.140776 \\
\hline $\mathrm{C}$ & -1.054428 & -2.521890 & -0.130841 \\
\hline $\mathrm{C}$ & -1.749302 & -3.022734 & 0.900389 \\
\hline $\mathrm{H}$ & -1.676716 & -2.597867 & 1.903926 \\
\hline $\mathrm{C}$ & -1.070699 & -3.206649 & -1.473012 \\
\hline $\mathrm{H}$ & -1.705727 & -2.659994 & -2.187220 \\
\hline $\mathrm{H}$ & -0.061293 & -3.244777 & -1.910812 \\
\hline C & -1.610593 & -4.624665 & -1.304920 \\
\hline $\mathrm{H}$ & -0.863215 & -5.244898 & -0.776322 \\
\hline $\mathrm{H}$ & -1.820490 & -5.090726 & -2.273286 \\
\hline O & -2.823029 & -4.596325 & -0.589844 \\
\hline $\mathrm{C}$ & -2.625882 & -4.230064 & 0.743374 \\
\hline $\mathrm{H}$ & -3.615431 & -4.021490 & 1.174973 \\
\hline $\mathrm{H}$ & -2.197271 & -5.070939 & 1.327252 \\
\hline $\mathrm{O}$ & 1.051027 & -2.199618 & 1.973350 \\
\hline B & 0.944164 & -2.575280 & 0.659968 \\
\hline $\mathrm{O}$ & 1.543012 & -3.754878 & 0.346888 \\
\hline $\mathrm{C}$ & 1.942618 & -4.351998 & 1.619158 \\
\hline $\mathrm{C}$ & 2.025186 & -3.109380 & 2.566505 \\
\hline C & 3.367191 & -2.396685 & 2.516766 \\
\hline $\mathrm{H}$ & 3.675713 & -2.180191 & 1.484784 \\
\hline $\mathrm{H}$ & 4.155992 & -2.987309 & 2.998420 \\
\hline $\mathrm{H}$ & 3.288661 & -1.440346 & 3.051143 \\
\hline C & 1.614856 & -3.363584 & 3.999715 \\
\hline $\mathrm{H}$ & 0.584967 & -3.729800 & 4.072438 \\
\hline $\mathrm{H}$ & 1.683819 & -2.427797 & 4.570301 \\
\hline $\mathrm{H}$ & 2.281769 & -4.095412 & 4.474766 \\
\hline $\mathrm{C}$ & 3.254944 & -5.072112 & 1.405000 \\
\hline $\mathrm{H}$ & 4.008997 & -4.415835 & 0.956548 \\
\hline $\mathrm{H}$ & 3.105318 & -5.925833 & 0.733659 \\
\hline $\mathrm{H}$ & 3.644612 & -5.456938 & 2.357136 \\
\hline $\mathrm{C}$ & 0.853857 & -5.335330 & 2.010635 \\
\hline $\mathrm{H}$ & -0.104925 & -4.831152 & 2.188944 \\
\hline $\mathrm{H}$ & 1.124166 & -5.889898 & 2.917570 \\
\hline $\mathrm{H}$ & 0.715451 & -6.060265 & 1.198870 \\
\hline $\mathrm{H}$ & 1.238039 & -1.741797 & -0.346279 \\
\hline $\mathrm{H}$ & -1.463985 & 4.465152 & -2.774396 \\
\hline
\end{tabular}

SCF energy: -3329.867407310

M06-D3 (Gas Phase)/6-31G(d)+SDD (Pd, Fe) opt. 1 imaginary frequency: $\quad 211 \mathrm{i} \mathrm{cm-1}$ 


\begin{tabular}{|c|c|c|c|}
\hline \multicolumn{4}{|c|}{ TS-2STania_conf 3} \\
\hline $\mathrm{P}$ & 1.655219 & 1.120019 & -0.134749 \\
\hline $\mathrm{P}$ & -1.938622 & 0.426301 & 0.578081 \\
\hline $\mathrm{C}$ & 3.377723 & 0.533167 & -0.394401 \\
\hline $\mathrm{C}$ & 6.027685 & -0.271256 & -0.810557 \\
\hline $\mathrm{C}$ & 3.669622 & -0.556244 & -1.220778 \\
\hline $\mathrm{C}$ & 4.433554 & 1.208410 & 0.232134 \\
\hline $\mathrm{C}$ & 5.748769 & 0.807952 & 0.023076 \\
\hline $\mathrm{C}$ & 4.985959 & -0.953475 & -1.432019 \\
\hline $\mathrm{H}$ & 2.869667 & -1.117285 & -1.703487 \\
\hline $\mathrm{H}$ & 4.230135 & 2.050244 & 0.893985 \\
\hline $\mathrm{H}$ & 6.558071 & 1.338663 & 0.520204 \\
\hline $\mathrm{H}$ & 5.194390 & -1.808045 & -2.073126 \\
\hline $\mathrm{H}$ & 7.056898 & -0.586538 & -0.968594 \\
\hline C & -3.425507 & 0.408461 & -0.479727 \\
\hline $\mathrm{C}$ & -5.678566 & 0.395579 & -2.131181 \\
\hline C & -4.627335 & 0.978547 & -0.038163 \\
\hline C & -3.363719 & -0.162906 & -1.753556 \\
\hline $\mathrm{C}$ & -4.485809 & -0.165841 & -2.580089 \\
\hline $\mathrm{C}$ & -5.750196 & 0.963847 & -0.858976 \\
\hline $\mathrm{H}$ & -2.429229 & -0.615014 & -2.091823 \\
\hline $\mathrm{H}$ & -4.433127 & -0.624147 & -3.566398 \\
\hline $\mathrm{H}$ & -6.685090 & 1.394912 & -0.506337 \\
\hline $\mathrm{H}$ & -6.559687 & 0.382774 & -2.769440 \\
\hline $\mathrm{C}$ & -2.402717 & -0.423124 & 2.121758 \\
\hline C & -2.895471 & -1.939614 & 4.415797 \\
\hline $\mathrm{C}$ & -3.629934 & -1.072832 & 2.285390 \\
\hline C & -1.417850 & -0.552912 & 3.110322 \\
\hline $\mathrm{C}$ & -1.668598 & -1.297646 & 4. 255294 \\
\hline C & -3.871851 & -1.826963 & 3.430692 \\
\hline $\mathrm{H}$ & -4.395028 & -1.017032 & 1.512550 \\
\hline $\mathrm{H}$ & -0.448017 & -0.071441 & 2.983897 \\
\hline $\mathrm{H}$ & -0.898971 & -1.382701 & 5.021191 \\
\hline $\mathrm{H}$ & -4.828971 & -2.330377 & 3.550064 \\
\hline $\mathrm{H}$ & -3.089313 & -2.530028 & 5.308894 \\
\hline $\mathrm{C}$ & -1.738737 & 2.209008 & 1.035917 \\
\hline C & -1.244856 & 4.859660 & 1.842396 \\
\hline $\mathrm{C}$ & -1.954616 & 2.615919 & 2.357098 \\
\hline $\mathrm{C}$ & -1.281522 & 3.164662 & 0.107445 \\
\hline $\mathrm{C}$ & -1.017541 & 4.469476 & 0.528934 \\
\hline $\mathrm{C}$ & -1.723564 & 3.928074 & 2.756017 \\
\hline $\mathrm{H}$ & -2.307030 & 1.894923 & 3.091964 \\
\hline $\mathrm{H}$ & -0.616188 & 5.195373 & -0.182731 \\
\hline $\mathrm{H}$ & -1.907781 & 4.216406 & 3.788600 \\
\hline $\mathrm{H}$ & -1.040665 & 5.882585 & 2.151248 \\
\hline $\mathrm{C}$ & 1.751949 & 1.840801 & 1.535123 \\
\hline $\mathrm{C}$ & 1.970345 & 2.776641 & 4.150887 \\
\hline C & 1.812018 & 0.935191 & 2.602786 \\
\hline $\mathrm{C}$ & 1.830511 & 3.211382 & 1.780774 \\
\hline C & 1.935684 & 3.675884 & 3.089887 \\
\hline C & 1.916681 & 1.405183 & 3.905975 \\
\hline $\mathrm{H}$ & 1.784769 & -0.141707 & 2.409053 \\
\hline $\mathrm{H}$ & 1.819500 & 3.919235 & 0.950870 \\
\hline $\mathrm{H}$ & 1.998698 & 4.746193 & 3.279614 \\
\hline $\mathrm{H}$ & 1.965801 & 0.698515 & 4.733553 \\
\hline $\mathrm{H}$ & 2.055928 & 3.143849 & 5.171595 \\
\hline $\mathrm{H}$ & -4.684572 & 1.435399 & 0.951616 \\
\hline $\mathrm{H}$ & 3.661827 & 2.842413 & -1.805792 \\
\hline C & 2.623973 & 3.126708 & -1.948255 \\
\hline C & 1.504421 & 2.504918 & -1.304421 \\
\hline $\mathrm{H}$ & 2.750834 & 4.702828 & -3.526187 \\
\hline C & 0.306073 & 3.116916 & -1.842207 \\
\hline C & 0.727448 & 4.110973 & -2.788224 \\
\hline $\mathrm{H}$ & 0.102320 & 4.744143 & -3.414533 \\
\hline C & 2.146194 & 4.102140 & -2.855381 \\
\hline $\mathrm{Fe}$ & 1.384359 & 2.250821 & -3.309737 \\
\hline $\mathrm{H}$ & 1.264049 & -0.540322 & -2.948658 \\
\hline $\mathrm{C}$ & 1.353395 & 0.239445 & -3.700464 \\
\hline C & 2.559467 & 0.826524 & -4.179955 \\
\hline $\mathrm{H}$ & -0.786551 & 0.641492 & -4.188970 \\
\hline $\mathrm{H}$ & 3.563049 & 0.589394 & -3.840430 \\
\hline $\mathrm{C}$ & 2.209864 & 1.826786 & -5.130132 \\
\hline $\mathrm{H}$ & 2.901324 & 2.480892 & -5.650355 \\
\hline
\end{tabular}

\begin{tabular}{|c|c|c|c|}
\hline $\mathrm{C}$ & 0.790752 & 1.862515 & -5.233400 \\
\hline $\mathrm{H}$ & 0.222360 & 2.532888 & -5.871302 \\
\hline $\mathrm{C}$ & 0.262551 & 0.877791 & -4.347907 \\
\hline $\mathrm{C}$ & -1.095357 & 2.841871 & -1.356096 \\
\hline $\mathrm{H}$ & -1.373089 & 1.798640 & -1.561027 \\
\hline $\mathrm{N}$ & -2.098869 & 3.664859 & -2.200494 \\
\hline $\mathrm{C}$ & -3.457995 & 3.843078 & -1.596593 \\
\hline $\mathrm{H}$ & -3.375687 & 4.326233 & -0.621970 \\
\hline $\mathrm{H}$ & -3.923898 & 2.859605 & -1.492484 \\
\hline $\mathrm{H}$ & -4.044787 & 4.464817 & -2.278152 \\
\hline $\mathrm{C}$ & -2.254449 & 3.109009 & -3.581163 \\
\hline $\mathrm{H}$ & -1.266173 & 2.937905 & -4.012695 \\
\hline $\mathrm{H}$ & -2.829634 & 3.816896 & -4.184249 \\
\hline $\mathrm{H}$ & -2.803369 & 2.164576 & -3.492867 \\
\hline $\mathrm{Pd}$ & 0.012632 & -0.650064 & -0.238393 \\
\hline $\mathrm{C}$ & -0.988546 & -2.495080 & -0.447432 \\
\hline $\mathrm{C}$ & -1.704057 & -3.125644 & 0.491341 \\
\hline $\mathrm{H}$ & -1.695844 & -2.803572 & 1.533005 \\
\hline $\mathrm{C}$ & -1.003023 & -2.978297 & -1.874055 \\
\hline $\mathrm{H}$ & -1.010399 & -2.144452 & -2.596064 \\
\hline $\mathrm{H}$ & -0.086118 & -3.553369 & -2.080387 \\
\hline C & -2.232802 & -3.856505 & -2.092631 \\
\hline $\mathrm{H}$ & -2.185546 & -4.374174 & -3.056576 \\
\hline $\mathrm{H}$ & -3.149775 & -3.233321 & -2.079126 \\
\hline O & -2.306391 & -4.848796 & -1.102041 \\
\hline $\mathrm{C}$ & -2.549264 & -4.321467 & 0.167491 \\
\hline $\mathrm{H}$ & -3.614970 & -4.024930 & 0.282519 \\
\hline $\mathrm{H}$ & -2.371165 & -5.130147 & 0.889699 \\
\hline $\mathrm{O}$ & 1.090844 & -2.381778 & 1.711859 \\
\hline B & 0.984046 & -2.659039 & 0.374512 \\
\hline $\mathrm{O}$ & 1.544246 & -3.838015 & -0.011610 \\
\hline $\mathrm{C}$ & 2.342487 & -4.275061 & 1.132851 \\
\hline $\mathrm{C}$ & 1.601578 & -3.602335 & 2.328574 \\
\hline C & 2.478307 & -3.191853 & 3.490581 \\
\hline $\mathrm{H}$ & 3.263434 & -2.491644 & 3.183703 \\
\hline $\mathrm{H}$ & 2.951575 & -4.066943 & 3.955307 \\
\hline $\mathrm{H}$ & 1.861441 & -2.699104 & 4.255092 \\
\hline C & 0.409339 & -4.404488 & 2.821642 \\
\hline $\mathrm{H}$ & -0.214356 & -4.757944 & 1.990778 \\
\hline $\mathrm{H}$ & -0.208774 & -3.772587 & 3.474483 \\
\hline $\mathrm{H}$ & 0.731872 & -5.279207 & 3.399381 \\
\hline $\mathrm{C}$ & 3.742190 & -3.721703 & 0.918500 \\
\hline $\mathrm{H}$ & 3.752597 & -2.621623 & 0.921920 \\
\hline $\mathrm{H}$ & 4.113411 & -4.062529 & -0.055767 \\
\hline $\mathrm{H}$ & 4.436244 & -4.077344 & 1.690100 \\
\hline $\mathrm{C}$ & 2.363768 & -5.785943 & 1.159667 \\
\hline $\mathrm{H}$ & 1.355588 & -6.211825 & 1.140064 \\
\hline $\mathrm{H}$ & 2.881353 & -6.146048 & 2.059140 \\
\hline $\mathrm{H}$ & 2.906348 & -6.165324 & 0.285978 \\
\hline $\mathrm{H}$ & 1.314622 & -1.726350 & -0.541399 \\
\hline $\mathrm{H}$ & -1.683239 & 4.600710 & -2.281712 \\
\hline
\end{tabular}

SCF energy: -3329.864281610

M06-D3 (Gas Phase)/6-31G(d)+SDD (Pd, Fe) opt. 1 imaginary frequency: $\quad 207 \mathrm{i} \mathrm{cm-1}$ 


\begin{tabular}{|c|c|c|c|}
\hline \multicolumn{4}{|c|}{ Int-4STania_conf1 } \\
\hline $\mathrm{P}$ & -2.166156 & 0.281725 & -0.343700 \\
\hline $\mathrm{C}$ & 2.721625 & 1.146447 & 1.917438 \\
\hline $\mathrm{C}$ & 4.551945 & 1.265050 & 4.022265 \\
\hline C & 2.505193 & 0.415245 & 3.088090 \\
\hline C & 3.844091 & 1.977445 & 1.828758 \\
\hline C & 4.759469 & 2.026507 & 2.873501 \\
\hline $\mathrm{C}$ & 3.417348 & 0.467416 & 4.136832 \\
\hline $\mathrm{H}$ & 1.604601 & -0.198963 & 3.184618 \\
\hline $\mathrm{H}$ & 3.993543 & 2.604732 & 0.948990 \\
\hline $\mathrm{H}$ & 5.632174 & 2.671859 & 2.798315 \\
\hline $\mathrm{H}$ & 3.239932 & -0.105581 & 5.044526 \\
\hline $\mathrm{H}$ & 5.268458 & 1.309944 & 4.839702 \\
\hline C & -2.836123 & -1.186313 & -1.211713 \\
\hline $\mathrm{C}$ & -3.795829 & -3.408951 & -2.615175 \\
\hline C & -3.328011 & -1.041373 & -2.522074 \\
\hline C & -2.850886 & -2.448824 & -0.611133 \\
\hline C & -3.331886 & -3.554033 & -1.310530 \\
\hline $\mathrm{C}$ & -3.799574 & -2.151125 & -3.219511 \\
\hline $\mathrm{H}$ & -2.496269 & -2.559708 & 0.413114 \\
\hline $\mathrm{H}$ & -3.346181 & -4.530885 & -0.831434 \\
\hline $\mathrm{H}$ & -4.187149 & -2.029979 & -4.229381 \\
\hline $\mathrm{H}$ & -4.170154 & -4.273603 & -3.159434 \\
\hline C & -3.579204 & 1.254790 & 0.223825 \\
\hline $\mathrm{C}$ & -5.694849 & 2.729856 & 1.268652 \\
\hline $\mathrm{C}$ & -4.895605 & 0.843113 & -0.004767 \\
\hline C & -3.324781 & 2.402508 & 0.988315 \\
\hline C & -4.382404 & 3.141360 & 1.499812 \\
\hline C & -5.949830 & 1.585410 & 0.519565 \\
\hline $\mathrm{H}$ & -5.104065 & -0.059119 & -0.578216 \\
\hline $\mathrm{H}$ & -2.295906 & 2.715543 & 1.177092 \\
\hline $\mathrm{H}$ & -4.185447 & 4.034684 & 2.088456 \\
\hline $\mathrm{H}$ & -6.974214 & 1.264475 & 0.343902 \\
\hline $\mathrm{H}$ & -6.522519 & 3.303930 & 1.679677 \\
\hline $\mathrm{C}$ & -1.379943 & 1.213071 & -1.718188 \\
\hline $\mathrm{C}$ & -0.160453 & 2.718956 & -3.741808 \\
\hline C & -1.877740 & 2.469667 & -2.073796 \\
\hline C & -0.251349 & 0.701439 & -2.394706 \\
\hline C & 0.352822 & 1.477041 & -3.385307 \\
\hline $\mathrm{C}$ & -1.279987 & 3.214065 & -3.085865 \\
\hline $\mathrm{H}$ & -2.744217 & 2.876180 & -1.555649 \\
\hline $\mathrm{H}$ & 1.259680 & 1.112097 & -3.869755 \\
\hline $\mathrm{H}$ & -1.684351 & 4.189440 & -3.347105 \\
\hline $\mathrm{H}$ & 0.325921 & 3.303982 & -4.519378 \\
\hline C & 1.282835 & 2.760588 & 0.048096 \\
\hline C & 0.870205 & 5.452615 & -0.557712 \\
\hline C & 0.452932 & 3.547757 & 0.855232 \\
\hline $\mathrm{C}$ & 1.910059 & 3.332837 & -1.058946 \\
\hline C & 1.702157 & 4.677313 & -1.358461 \\
\hline C & 0.246538 & 4.887807 & 0.553893 \\
\hline $\mathrm{H}$ & -0.013843 & 3.108648 & 1.741551 \\
\hline $\mathrm{H}$ & 2.567635 & 2.731886 & -1.688677 \\
\hline $\mathrm{H}$ & 2.199832 & 5.121804 & -2.218722 \\
\hline $\mathrm{H}$ & -0.390953 & 5.497510 & 1.191540 \\
\hline $\mathrm{H}$ & 0.714877 & 6.503775 & -0.791978 \\
\hline $\mathrm{H}$ & -3.343732 & -0.058061 & -2.995688 \\
\hline $\mathrm{H}$ & 4.570591 & 0.712186 & -0.371878 \\
\hline C & 3.840549 & 0.247995 & -1.027264 \\
\hline C & 2.424191 & 0.230186 & -0.812664 \\
\hline $\mathrm{H}$ & 5.125340 & -0.752022 & -2.558790 \\
\hline C & 1.842310 & -0.575311 & -1.870390 \\
\hline C & 2.912497 & -1.029656 & -2.702755 \\
\hline $\mathrm{H}$ & 2.846064 & -1.688995 & -3.563073 \\
\hline C & 4.135027 & -0.528554 & -2.176543 \\
\hline $\mathrm{Fe}$ & 3.166888 & -1.646069 & -0.766229 \\
\hline $\mathrm{H}$ & 2.385469 & -1.899349 & 1.930817 \\
\hline C & 2.902603 & -2.403225 & 1.122302 \\
\hline C & 4.286560 & -2.271338 & 0.822643 \\
\hline $\mathrm{H}$ & 1.276821 & -3.572765 & 0.136440 \\
\hline $\mathrm{H}$ & 4.991253 & -1.634399 & 1.349397 \\
\hline C & 4.562174 & -3.067871 & -0.324817 \\
\hline $\mathrm{H}$ & 5.520299 & -3.153550 & -0.826306 \\
\hline C & 3.346742 & -3.683996 & -0.738229 \\
\hline
\end{tabular}

\begin{tabular}{|c|c|c|c|}
\hline $\mathrm{H}$ & 3.235224 & -4.348137 & -1.589933 \\
\hline C & 2.319657 & -3.270162 & 0.159672 \\
\hline $\mathrm{C}$ & 0.352917 & -0.640480 & -2.031530 \\
\hline $\mathrm{H}$ & -0.054947 & -0.964381 & -1.060794 \\
\hline $\mathrm{N}$ & -0.183681 & -1.730849 & -2.977691 \\
\hline C & 0.212119 & -3.107936 & -2.563502 \\
\hline $\mathrm{H}$ & -0.112988 & -3.260213 & -1.530549 \\
\hline $\mathrm{H}$ & 1.297518 & -3.210008 & -2.631107 \\
\hline $\mathrm{H}$ & -0.291557 & -3.824335 & -3.218637 \\
\hline C & 0.012075 & -1.518386 & -4.442482 \\
\hline $\mathrm{H}$ & -0.405187 & -0.549093 & -4.723472 \\
\hline $\mathrm{H}$ & -0.519601 & -2.316029 & -4.969602 \\
\hline $\mathrm{H}$ & 1.075416 & -1.557032 & -4.686766 \\
\hline $\mathrm{Pd}$ & -0.570488 & -0.099173 & 1.287748 \\
\hline $\mathrm{H}$ & -1.205536 & -1.673642 & -2.829369 \\
\hline $\mathrm{P}$ & 1.515827 & 1.022248 & 0.554131 \\
\hline C & -1.945543 & -1.113242 & 2.343840 \\
\hline C & -3.165792 & -0.836773 & 2.782314 \\
\hline $\mathrm{H}$ & -3.771283 & -0.009457 & 2.408638 \\
\hline C & -1.085858 & -2.212187 & 2.888741 \\
\hline $\mathrm{H}$ & -1.051393 & -3.079403 & 2.210790 \\
\hline $\mathrm{H}$ & -0.034961 & -1.894694 & 3.051698 \\
\hline $\mathrm{C}$ & -1.676982 & -2.620514 & 4.255814 \\
\hline $\mathrm{H}$ & -1.482344 & -1.822857 & 4.994423 \\
\hline $\mathrm{H}$ & -1.218161 & -3.550402 & 4.608072 \\
\hline 0 & -3.045686 & -2.854151 & 4.113372 \\
\hline C & -3.766952 & -1.676023 & 3.889279 \\
\hline $\mathrm{H}$ & -4.789623 & -1.975433 & 3.628059 \\
\hline $\mathrm{H}$ & -3.824636 & -1.066268 & 4.810972 \\
\hline
\end{tabular}

SCF energy: -2918.234232650

M06-D3 (Gas Phase) /6-31G(d) +SDD (Pd, Fe) opt. No imaginary frequency 


\begin{tabular}{|c|c|c|c|}
\hline \multicolumn{4}{|c|}{ Int-4STania_conf 2} \\
\hline $\mathrm{P}$ & 1.478683 & 1.166895 & 0.294483 \\
\hline $\mathrm{P}$ & -2.130255 & 0.088964 & -0.318756 \\
\hline $\mathrm{C}$ & 2.674627 & 1.549858 & 1.616674 \\
\hline C & 4.471889 & 2.069467 & 3.687729 \\
\hline C & 2.469792 & 1.004932 & 2.887393 \\
\hline C & 3.766629 & 2.398815 & 1.404187 \\
\hline $\mathrm{C}$ & 4.666360 & 2.647029 & 2.434094 \\
\hline C & 3.365789 & 1.257617 & 3.920850 \\
\hline $\mathrm{H}$ & 1.587979 & 0.382837 & 3.077879 \\
\hline $\mathrm{H}$ & 3.902273 & 2.884714 & 0.436819 \\
\hline $\mathrm{H}$ & 5.516013 & 3.305006 & 2.263943 \\
\hline $\mathrm{H}$ & 3.197701 & 0.830502 & 4.907250 \\
\hline $\mathrm{H}$ & 5.175972 & 2.270685 & 4.492366 \\
\hline $\mathrm{C}$ & -2.926286 & -1.466020 & -0.833974 \\
\hline C & -4.145503 & -3.833559 & -1.668968 \\
\hline C & -3.892699 & -1.440735 & -1.850054 \\
\hline C & -2.573770 & -2.683508 & -0.247418 \\
\hline C & -3.178120 & -3.866396 & -0.668164 \\
\hline $\mathrm{C}$ & -4.504817 & -2.620775 & -2.257428 \\
\hline $\mathrm{H}$ & -1.828689 & -2.697811 & 0.548719 \\
\hline $\mathrm{H}$ & -2.909075 & -4.810788 & -0.198904 \\
\hline $\mathrm{H}$ & -5.266266 & -2.595261 & -3.034231 \\
\hline $\mathrm{H}$ & -4.628832 & -4.754716 & -1.987848 \\
\hline $\mathrm{C}$ & -3.450456 & 1.203474 & 0.230721 \\
\hline $\mathrm{C}$ & -5.363910 & 2.914061 & 1. 321554 \\
\hline C & -4.763474 & 0.759137 & 0.415970 \\
\hline C & -3.096850 & 2.505256 & 0.615123 \\
\hline C & -4.053248 & 3.357864 & 1.148182 \\
\hline C & -5.715622 & 1.618614 & 0.957336 \\
\hline $\mathrm{H}$ & -5.046741 & -0.259548 & 0.156525 \\
\hline $\mathrm{H}$ & -2.071094 & 2.854181 & 0.483354 \\
\hline $\mathrm{H}$ & -3.775396 & 4.370070 & 1.434866 \\
\hline $\mathrm{H}$ & -6.736137 & 1.269212 & 1.098030 \\
\hline $\mathrm{H}$ & -6.111222 & 3.580844 & 1.746320 \\
\hline $\mathrm{C}$ & -1.461768 & 0.789365 & -1.894553 \\
\hline $\mathrm{C}$ & -0.472170 & 2.002341 & -4.232609 \\
\hline $\mathrm{C}$ & -2.072443 & 1.926142 & -2.437134 \\
\hline $\mathrm{C}$ & -0.332037 & 0.254593 & -2.550691 \\
\hline $\mathrm{C}$ & 0.154297 & 0.883213 & -3.698789 \\
\hline $\mathrm{C}$ & -1.594184 & 2.521329 & -3.599170 \\
\hline $\mathrm{H}$ & -2.942160 & 2.357768 & -1.946292 \\
\hline $\mathrm{H}$ & 1.056800 & 0.498069 & -4.179184 \\
\hline $\mathrm{H}$ & -2.095421 & 3.399930 & -3.998969 \\
\hline $\mathrm{H}$ & -0.077401 & 2.471468 & -5.131174 \\
\hline $\mathrm{C}$ & 1.161951 & 2.792111 & -0.470678 \\
\hline $\mathrm{C}$ & 0.587045 & 5.317105 & -1.508291 \\
\hline $\mathrm{C}$ & 0.393473 & 3.701031 & 0.267137 \\
\hline $\mathrm{C}$ & 1.657714 & 3.164305 & -1.720252 \\
\hline $\mathrm{C}$ & 1.368728 & 4.425981 & -2.235500 \\
\hline $\mathrm{C}$ & 0.104048 & 4.956673 & -0.250956 \\
\hline $\mathrm{H}$ & 0.038713 & 3.426829 & 1.264373 \\
\hline $\mathrm{H}$ & 2.277532 & 2.473247 & -2.292918 \\
\hline $\mathrm{H}$ & 1.763153 & 4.714856 & -3.208303 \\
\hline $\mathrm{H}$ & -0.487726 & 5.661769 & 0.330159 \\
\hline $\mathrm{H}$ & 0.365589 & 6.302447 & -1.913160 \\
\hline $\mathrm{H}$ & -4.170322 & -0.496034 & -2.320588 \\
\hline $\mathrm{H}$ & 4.530847 & 0.851711 & -0.581649 \\
\hline $\mathrm{C}$ & 3.825701 & 0.266107 & -1.162535 \\
\hline $\mathrm{C}$ & 2.410191 & 0.225432 & -0.948095 \\
\hline $\mathrm{H}$ & 5.158818 & -0.893292 & -2.532072 \\
\hline $\mathrm{C}$ & 1.863211 & -0.751344 & -1.865123 \\
\hline $\mathrm{C}$ & 2.960306 & -1.279304 & -2.625811 \\
\hline $\mathrm{H}$ & 2.937116 & -2.066863 & -3.376617 \\
\hline $\mathrm{C}$ & 4.159025 & -0.656628 & -2.184272 \\
\hline $\mathrm{Fe}$ & 3.217960 & -1.597565 & -0.626930 \\
\hline $\mathrm{H}$ & 2.346859 & -1.483614 & 2.045368 \\
\hline $\mathrm{C}$ & 2.910820 & -2.082756 & 1.338862 \\
\hline $\mathrm{C}$ & 4.300679 & -1.943063 & 1.069298 \\
\hline $\mathrm{H}$ & 1.356769 & -3.433303 & 0.467577 \\
\hline $\mathrm{H}$ & 4.963171 & -1.210125 & 1.520257 \\
\hline $\mathrm{C}$ & 4.645878 & -2.885805 & 0.060269 \\
\hline $\mathrm{H}$ & 5.624650 & -3.007813 & -0.391078 \\
\hline
\end{tabular}

\begin{tabular}{|c|c|c|c|}
\hline C & 3.468571 & -3.603020 & -0.295944 \\
\hline $\mathrm{H}$ & 3.413537 & -4.387838 & -1.044561 \\
\hline $\mathrm{C}$ & 2.392393 & -3.102422 & 0.495299 \\
\hline C & 0.386793 & -0.989760 & -2.078218 \\
\hline $\mathrm{H}$ & -0.076403 & -1.378854 & -1.159400 \\
\hline $\mathrm{N}$ & 0.210761 & -2.131677 & -3.105669 \\
\hline C & -1.124990 & -2.205686 & -3.777203 \\
\hline $\mathrm{H}$ & -1.341250 & -1.263548 & -4.283578 \\
\hline $\mathrm{H}$ & -1.884637 & -2.409997 & -3.019254 \\
\hline $\mathrm{H}$ & -1.088149 & -3.024019 & -4.501370 \\
\hline $\mathrm{C}$ & 0.531164 & -3.463134 & -2.501604 \\
\hline $\mathrm{H}$ & 1.484215 & -3.394129 & -1.971837 \\
\hline $\mathrm{H}$ & 0.577001 & -4.211842 & -3.297215 \\
\hline $\mathrm{H}$ & -0.278626 & -3.710142 & -1.806169 \\
\hline $\mathrm{Pd}$ & -0.447426 & -0.004336 & 1.253165 \\
\hline $\mathrm{C}$ & -1.719927 & -0.909930 & 2.516316 \\
\hline $\mathrm{C}$ & -2.809524 & -0.398772 & 3.075763 \\
\hline $\mathrm{H}$ & -3.349373 & 0.458125 & 2.669308 \\
\hline $\mathrm{C}$ & -0.940368 & -2.048804 & 3.099125 \\
\hline $\mathrm{H}$ & -1.141018 & -2.993352 & 2.570763 \\
\hline $\mathrm{H}$ & 0.154572 & -1.886048 & 3.054365 \\
\hline $\mathrm{C}$ & -1.351882 & -2.174380 & 4.579573 \\
\hline $\mathrm{H}$ & -0.938277 & -1.323239 & 5.150850 \\
\hline $\mathrm{H}$ & -0.966578 & -3.106193 & 5.007370 \\
\hline 0 & -2.744522 & -2.214517 & 4.674300 \\
\hline $\mathrm{C}$ & -3.335445 & -0.984003 & 4.367046 \\
\hline $\mathrm{H}$ & -4.415793 & -1.160940 & 4.292419 \\
\hline $\mathrm{H}$ & -3.174809 & -0.253458 & 5.183130 \\
\hline $\mathrm{H}$ & 0.903577 & -1.944776 & -3.840706 \\
\hline
\end{tabular}

SCF energy: -2918.232913480

M06-D3 (Gas Phase) /6-31G(d) +SDD (Pd, Fe) opt. No imaginary frequency 


\begin{tabular}{|c|c|c|c|}
\hline \multicolumn{4}{|c|}{ Int-4STania_conf 3} \\
\hline $\mathrm{P}$ & 1.504869 & 1.195702 & 0.304562 \\
\hline $\mathrm{P}$ & -2.099542 & 0.131492 & -0.330374 \\
\hline $\mathrm{C}$ & 2.661978 & 1.517058 & 1.675720 \\
\hline C & 4.378160 & 1.934930 & 3.835904 \\
\hline C & 2.374018 & 0.966791 & 2.928295 \\
\hline C & 3.798175 & 2.319729 & 1.525560 \\
\hline $\mathrm{C}$ & 4.657103 & 2.517363 & 2.600398 \\
\hline C & 3.228655 & 1.168677 & 4.006584 \\
\hline $\mathrm{H}$ & 1.457715 & 0.381088 & 3.072438 \\
\hline $\mathrm{H}$ & 4.000274 & 2.809216 & 0.571587 \\
\hline $\mathrm{H}$ & 5.541318 & 3.139730 & 2.479655 \\
\hline $\mathrm{H}$ & 2.994758 & 0.738690 & 4.978142 \\
\hline $\mathrm{H}$ & 5.050778 & 2.097031 & 4.675543 \\
\hline $\mathrm{C}$ & -2.927396 & -1.387997 & -0.900903 \\
\hline C & -4.203453 & -3.693697 & -1.821354 \\
\hline C & -3.864930 & -1.307605 & -1.940826 \\
\hline C & -2.634276 & -2.629123 & -0.331450 \\
\hline C & -3.266373 & -3.781025 & -0.795326 \\
\hline $\mathrm{C}$ & -4.505556 & -2.456666 & -2.390991 \\
\hline $\mathrm{H}$ & -1.913078 & -2.688073 & 0.483096 \\
\hline $\mathrm{H}$ & -3.040933 & -4.744282 & -0.341472 \\
\hline $\mathrm{H}$ & -5.244497 & -2.387798 & -3.186706 \\
\hline $\mathrm{H}$ & -4.707846 & -4.590657 & -2.174696 \\
\hline $\mathrm{C}$ & -3.401121 & 1.254892 & 0.244133 \\
\hline $\mathrm{C}$ & -5.292547 & 2.963514 & 1.375351 \\
\hline C & -4.724903 & 0.831279 & 0.398857 \\
\hline C & -3.026017 & 2.535180 & 0.678343 \\
\hline C & -3.971510 & 3.386855 & 1.231866 \\
\hline C & -5.666061 & 1.689709 & 0.960409 \\
\hline $\mathrm{H}$ & -5.025496 & -0.171151 & 0.098854 \\
\hline $\mathrm{H}$ & -1.991934 & 2.868020 & 0.570662 \\
\hline $\mathrm{H}$ & -3.677714 & 4.382010 & 1.558950 \\
\hline $\mathrm{H}$ & -6.694918 & 1.356419 & 1.077290 \\
\hline $\mathrm{H}$ & -6.030942 & 3.629452 & 1.816687 \\
\hline $\mathrm{C}$ & -1.403311 & 0.868035 & -1.877331 \\
\hline $\mathrm{C}$ & -0.351633 & 2.146187 & -4.152459 \\
\hline $\mathrm{C}$ & -1.982758 & 2.036158 & -2.386288 \\
\hline $\mathrm{C}$ & -0.274574 & 0.334744 & -2.536139 \\
\hline $\mathrm{C}$ & 0.242971 & 0.994478 & -3.652251 \\
\hline $\mathrm{C}$ & -1.472965 & 2.664391 & -3.517320 \\
\hline $\mathrm{H}$ & -2.851102 & 2.467587 & -1.892789 \\
\hline $\mathrm{H}$ & 1.144898 & 0.607910 & -4.132880 \\
\hline $\mathrm{H}$ & -1.948879 & 3.568201 & -3.890982 \\
\hline $\mathrm{H}$ & 0.068035 & 2.641567 & -5.025271 \\
\hline $\mathrm{C}$ & 1.278025 & 2.832845 & -0.463735 \\
\hline $\mathrm{C}$ & 0.844665 & 5.384887 & -1.502193 \\
\hline $\mathrm{C}$ & 0.509742 & 3.763571 & 0.246629 \\
\hline $\mathrm{C}$ & 1.842632 & 3.195067 & -1.686955 \\
\hline $\mathrm{C}$ & 1.624454 & 4.470694 & -2.202443 \\
\hline $\mathrm{C}$ & 0.291158 & 5.033142 & -0.271749 \\
\hline $\mathrm{H}$ & 0.103391 & 3.495938 & 1.225739 \\
\hline $\mathrm{H}$ & 2.461773 & 2.485452 & -2.237366 \\
\hline $\mathrm{H}$ & 2.073556 & 4.752818 & -3.153269 \\
\hline $\mathrm{H}$ & -0.299170 & 5.755799 & 0.288520 \\
\hline $\mathrm{H}$ & 0.679526 & 6.381310 & -1.906877 \\
\hline $\mathrm{H}$ & -4.098185 & -0.343686 & -2.396241 \\
\hline $\mathrm{H}$ & 4.567522 & 0.744681 & -0.449492 \\
\hline $\mathrm{C}$ & 3.862176 & 0.189535 & -1.059451 \\
\hline $\mathrm{C}$ & 2.438845 & 0.214311 & -0.905405 \\
\hline $\mathrm{H}$ & 5.198189 & -1.033106 & -2.369927 \\
\hline $\mathrm{C}$ & 1.887230 & -0.742781 & -1.840894 \\
\hline $\mathrm{C}$ & 2.990797 & -1.321361 & -2.554885 \\
\hline $\mathrm{H}$ & 2.964732 & -2.107811 & -3.306541 \\
\hline $\mathrm{C}$ & 4.196435 & -0.750995 & -2.064268 \\
\hline $\mathrm{Fe}$ & 3.147276 & -1.644036 & -0.547052 \\
\hline $\mathrm{H}$ & 2.009756 & -1.566297 & 2.028259 \\
\hline $\mathrm{C}$ & 2.649230 & -2.150065 & 1.373063 \\
\hline $\mathrm{C}$ & 4.059521 & -2.006079 & 1.243230 \\
\hline $\mathrm{H}$ & 1.194267 & -3.496975 & 0.341540 \\
\hline $\mathrm{H}$ & 4.675560 & -1.279660 & 1.764754 \\
\hline $\mathrm{C}$ & 4.503903 & -2.938751 & 0.263884 \\
\hline $\mathrm{H}$ & 5.522625 & -3.054034 & -0.090447 \\
\hline
\end{tabular}

\begin{tabular}{|c|c|c|c|}
\hline $\mathrm{C}$ & 3.369540 & -3.653523 & -0.214654 \\
\hline $\mathrm{H}$ & 3.387514 & -4.426629 & -0.977029 \\
\hline $\mathrm{C}$ & 2.220850 & -3.163049 & 0.472978 \\
\hline $\mathrm{C}$ & 0.410419 & -0.938806 & -2.095078 \\
\hline $\mathrm{H}$ & -0.081321 & -1.350184 & -1.201072 \\
\hline $\mathrm{N}$ & 0.233469 & -2.042100 & -3.164718 \\
\hline C & -1.082952 & -2.055493 & -3.876928 \\
\hline $\mathrm{H}$ & -1.253879 & -1.094487 & -4.364675 \\
\hline $\mathrm{H}$ & -1.871423 & -2.253714 & -3.146981 \\
\hline $\mathrm{H}$ & -1.050867 & -2.856386 & -4.620571 \\
\hline $\mathrm{C}$ & 0.495354 & -3.401311 & -2.594537 \\
\hline $\mathrm{H}$ & 1.429227 & -3.377889 & -2.027884 \\
\hline $\mathrm{H}$ & 0.550254 & -4.124951 & -3.412483 \\
\hline $\mathrm{H}$ & -0.346460 & -3.647393 & -1.937619 \\
\hline $\mathrm{Pd}$ & -0.424127 & 0.019079 & 1.243541 \\
\hline $\mathrm{C}$ & -1.689607 & -0.970261 & 2.455334 \\
\hline $\mathrm{C}$ & -2.775867 & -0.462414 & 3.028507 \\
\hline $\mathrm{H}$ & -3.308744 & 0.407151 & 2.639188 \\
\hline $\mathrm{C}$ & -1.003132 & -2.207117 & 2.966287 \\
\hline $\mathrm{H}$ & -0.678857 & -2.884135 & 2.159727 \\
\hline $\mathrm{H}$ & -0.102568 & -1.956516 & 3.548995 \\
\hline $\mathrm{C}$ & -2.003586 & -2.943976 & 3.876417 \\
\hline $\mathrm{H}$ & -1.511296 & -3.761491 & 4.413730 \\
\hline $\mathrm{H}$ & -2.813621 & -3.365201 & 3.249721 \\
\hline 0 & -2.515912 & -2.066886 & 4.833409 \\
\hline $\mathrm{C}$ & -3.345932 & -1.087471 & 4.278605 \\
\hline $\mathrm{H}$ & -4.339590 & -1.511027 & 4.029387 \\
\hline $\mathrm{H}$ & -3.502625 & -0.326908 & 5.053300 \\
\hline $\mathrm{H}$ & 0.954048 & -1.853160 & -3.872233 \\
\hline
\end{tabular}

SCF energy: -2918.231521790

M06-D3 (Gas Phase) /6-31G(d)+SDD (Pd, Fe) opt. No imaginary frequency 


\begin{tabular}{|c|c|c|c|}
\hline \multicolumn{4}{|c|}{ Int-4RTania_conf2 } \\
\hline $\mathrm{P}$ & -2.204026 & 0.046095 & 0.624733 \\
\hline $\mathrm{C}$ & 2.406948 & -1.990676 & -1.316502 \\
\hline $\mathrm{C}$ & 4.041420 & -3.058541 & -3.311639 \\
\hline C & 2.104425 & -1.786793 & -2.665550 \\
\hline C & 3.512869 & -2.777177 & -0.974643 \\
\hline C & 4.331256 & -3.298800 & -1.969691 \\
\hline $\mathrm{C}$ & 2.919940 & -2.312777 & -3.662117 \\
\hline $\mathrm{H}$ & 1.212824 & -1.215544 & -2.943773 \\
\hline $\mathrm{H}$ & 3.724424 & -3.000311 & 0.071960 \\
\hline $\mathrm{H}$ & 5.191801 & -3.906967 & -1.698805 \\
\hline $\mathrm{H}$ & 2.676667 & -2.149177 & -4.709884 \\
\hline $\mathrm{H}$ & 4.682231 & -3.472419 & -4.087347 \\
\hline C & -2.727523 & 1.793227 & 0.781005 \\
\hline $\mathrm{C}$ & -3.486687 & 4.467459 & 1.085662 \\
\hline C & -3.194283 & 2.260770 & 2.023021 \\
\hline C & -2.655677 & 2.674529 & -0.302238 \\
\hline C & -3.041169 & 4.005250 & -0.149673 \\
\hline $\mathrm{C}$ & -3.565392 & 3.595044 & 2.172260 \\
\hline $\mathrm{H}$ & -2.296075 & 2.314614 & -1.268659 \\
\hline $\mathrm{H}$ & -2.992331 & 4.681900 & -1.000451 \\
\hline $\mathrm{H}$ & -3.935057 & 3.949349 & 3.132727 \\
\hline $\mathrm{H}$ & -3.785641 & 5.507140 & 1.202466 \\
\hline $\mathrm{C}$ & -3.707221 & -0.956714 & 0.563242 \\
\hline $\mathrm{C}$ & -5.967653 & -2.554127 & 0.269873 \\
\hline $\mathrm{C}$ & -4.979039 & -0.378375 & 0.596981 \\
\hline C & -3.570327 & -2.339652 & 0.374727 \\
\hline C & -4.699741 & -3.135011 & 0.239444 \\
\hline C & -6.105886 & -1.181585 & 0.448470 \\
\hline $\mathrm{H}$ & -5.097729 & 0.696966 & 0.724410 \\
\hline $\mathrm{H}$ & -2.575919 & -2.789627 & 0.338476 \\
\hline $\mathrm{H}$ & -4.593779 & -4.208666 & 0.099913 \\
\hline $\mathrm{H}$ & -7.095459 & -0.730540 & 0.471024 \\
\hline $\mathrm{H}$ & -6.851487 & -3.177230 & 0.151249 \\
\hline C & -1.388958 & -0.280672 & 2.236311 \\
\hline $\mathrm{C}$ & -0.093872 & -0.931981 & 4.633381 \\
\hline C & -1.922134 & -1.242249 & 3.098871 \\
\hline C & -0.188462 & 0.373006 & 2.589188 \\
\hline C & 0.451731 & 0.017839 & 3.776962 \\
\hline $\mathrm{C}$ & -1.286089 & -1.558626 & 4.295290 \\
\hline $\mathrm{H}$ & -2.845170 & -1.755181 & 2.835258 \\
\hline $\mathrm{H}$ & 1.410278 & 0.476413 & 4.024467 \\
\hline $\mathrm{H}$ & -1.718817 & -2.309243 & 4.952839 \\
\hline $\mathrm{H}$ & 0.422452 & -1.191493 & 5.555047 \\
\hline C & 1.098267 & -2.601475 & 1.156253 \\
\hline $\mathrm{C}$ & 0.694704 & -4.768910 & 2.865966 \\
\hline C & 0.181546 & -3.605141 & 0.820602 \\
\hline $\mathrm{C}$ & 1.816802 & -2.696113 & 2.348631 \\
\hline C & 1.613595 & -3.779525 & 3.199872 \\
\hline C & -0.020394 & -4.683787 & 1.672322 \\
\hline $\mathrm{H}$ & -0.357595 & -3.549825 & -0.129312 \\
\hline $\mathrm{H}$ & 2.544225 & -1.927443 & 2.613261 \\
\hline $\mathrm{H}$ & 2.182671 & -3.853665 & 4.125005 \\
\hline $\mathrm{H}$ & -0.726119 & -5.466816 & 1.401352 \\
\hline $\mathrm{H}$ & 0.542496 & -5.616711 & 3.530805 \\
\hline $\mathrm{H}$ & -3.273123 & 1.577562 & 2.870934 \\
\hline $\mathrm{H}$ & 4.456290 & -0.774060 & 0.451478 \\
\hline C & 3.802287 & -0.037782 & 0.907625 \\
\hline C & 2.373332 & -0.020270 & 0.804285 \\
\hline $\mathrm{H}$ & 5.247001 & 1.414470 & 1.802136 \\
\hline C & 1.912175 & 1.180256 & 1.476512 \\
\hline C & 3.064842 & 1.865311 & 1.972815 \\
\hline $\mathrm{H}$ & 3.094720 & 2.818948 & 2.491533 \\
\hline C & 4.220541 & 1.117934 & 1.614444 \\
\hline $\mathrm{Fe}$ & 3.162184 & 1.624141 & -0.059145 \\
\hline $\mathrm{H}$ & 2.167885 & 0.789566 & -2.560485 \\
\hline C & 2.758708 & 1.554342 & -2.068857 \\
\hline C & 4.159896 & 1.484677 & -1.835597 \\
\hline $\mathrm{H}$ & 1.242045 & 3.106339 & -1.543319 \\
\hline $\mathrm{H}$ & 4.804440 & 0.650895 & -2.098726 \\
\hline C & 4.545339 & 2.665367 & -1.139603 \\
\hline $\mathrm{H}$ & 5.543023 & 2.898637 & -0.783270 \\
\hline C & 3.380850 & 3.459310 & -0.936050 \\
\hline
\end{tabular}

\begin{tabular}{|c|c|c|c|}
\hline $\mathrm{H}$ & 3.353950 & 4.418776 & -0.428536 \\
\hline C & 2.274377 & 2.768363 & -1.511495 \\
\hline $\mathrm{C}$ & 0.445090 & 1.409685 & 1.683642 \\
\hline $\mathrm{H}$ & -0.024553 & 1.351513 & 0.689393 \\
\hline $\mathrm{N}$ & 0.035208 & 2.821677 & 2.145102 \\
\hline C & 0.460555 & 3.894471 & 1.199471 \\
\hline $\mathrm{H}$ & 0.078722 & 3.646654 & 0.205847 \\
\hline $\mathrm{H}$ & 1.551133 & 3.947340 & 1.176524 \\
\hline $\mathrm{H}$ & 0.029162 & 4.841534 & 1.535716 \\
\hline C & 0.339906 & 3.189403 & 3.560213 \\
\hline $\mathrm{H}$ & -0.104566 & 2.449106 & 4.228308 \\
\hline $\mathrm{H}$ & -0.100111 & 4.172150 & 3.752608 \\
\hline $\mathrm{H}$ & 1.420641 & 3.231666 & 3.709149 \\
\hline $\mathrm{Pd}$ & -0.739994 & -0.411222 & -1.100419 \\
\hline $\mathrm{H}$ & -0.996513 & 2.786276 & 2.086200 \\
\hline $\mathrm{P}$ & 1.328382 & -1.247838 & -0.046013 \\
\hline C & -2.125750 & 0.121110 & -2.463969 \\
\hline C & -1.387371 & 0.732497 & -3.391688 \\
\hline $\mathrm{H}$ & -0.371371 & 1.107220 & -3.207842 \\
\hline C & -3.536397 & -0.292055 & -2.666844 \\
\hline $\mathrm{H}$ & -4.220968 & 0.439948 & -2.208611 \\
\hline $\mathrm{H}$ & -3.759348 & -1.268559 & -2.212507 \\
\hline $\mathrm{C}$ & -3.785905 & -0.354160 & -4.185540 \\
\hline $\mathrm{H}$ & -3.278034 & -1.239526 & -4.608075 \\
\hline $\mathrm{H}$ & -4.857577 & -0.434713 & -4.394077 \\
\hline 0 & -3.330288 & 0.818605 & -4.804127 \\
\hline $\mathrm{C}$ & -1.940272 & 0.917515 & -4.797908 \\
\hline $\mathrm{H}$ & -1.685108 & 1.914373 & -5.177688 \\
\hline $\mathrm{H}$ & -1.479666 & 0.171586 & -5.473408 \\
\hline
\end{tabular}

SCF energy: -2918.234572480

M06-D3 (Gas Phase) /6-31G(d) +SDD (Pd, Fe) opt. No imaginary frequency 


\begin{tabular}{|c|c|c|c|}
\hline \multicolumn{4}{|c|}{ Int-4STania_conf 4} \\
\hline $\mathrm{P}$ & -2.159316 & 0.240462 & -0.374684 \\
\hline $\mathrm{C}$ & 2.632985 & 1.201111 & 1.942897 \\
\hline $\mathrm{C}$ & 4.306830 & 1.237798 & 4.175052 \\
\hline C & 2.299602 & 0.479489 & 3.092706 \\
\hline C & 3.793668 & 1.981981 & 1.937535 \\
\hline C & 4.631252 & 1.989847 & 3.046944 \\
\hline $\mathrm{C}$ & 3.132696 & 0.490634 & 4.205990 \\
\hline $\mathrm{H}$ & 1.366230 & -0.096035 & 3.124141 \\
\hline $\mathrm{H}$ & 4.032386 & 2.602589 & 1.072701 \\
\hline $\mathrm{H}$ & 5.534819 & 2.595887 & 3.038794 \\
\hline $\mathrm{H}$ & 2.864249 & -0.073049 & 5.096907 \\
\hline $\mathrm{H}$ & 4.963405 & 1.251294 & 5.042463 \\
\hline C & -2.814514 & -1.232206 & -1.243042 \\
\hline $\mathrm{C}$ & -3.765600 & -3.466996 & -2.632939 \\
\hline C & -3.254296 & -1.106973 & -2.573513 \\
\hline C & -2.882842 & -2.478911 & -0.614876 \\
\hline C & -3.358571 & -3.590425 & -1.307274 \\
\hline $\mathrm{C}$ & -3.720702 & -2.223471 & -3.264111 \\
\hline $\mathrm{H}$ & -2.576499 & -2.569655 & 0.426160 \\
\hline $\mathrm{H}$ & -3.413224 & -4.555535 & -0.807636 \\
\hline $\mathrm{H}$ & -4.068914 & -2.117594 & -4.289951 \\
\hline $\mathrm{H}$ & -4.135304 & -4.336544 & -3.172438 \\
\hline $\mathrm{C}$ & -3.580595 & 1.200796 & 0.190282 \\
\hline $\mathrm{C}$ & -5.710086 & 2.646161 & 1.248100 \\
\hline $\mathrm{C}$ & -4.889674 & 0.744553 & 0.010754 \\
\hline C & -3.339489 & 2.378406 & 0.913492 \\
\hline C & -4.404703 & 3.102175 & 1.430973 \\
\hline C & -5.950913 & 1.472226 & 0.541062 \\
\hline $\mathrm{H}$ & -5.086742 & -0.179005 & -0.531996 \\
\hline $\mathrm{H}$ & -2.315585 & 2.726308 & 1.066241 \\
\hline $\mathrm{H}$ & -4.218665 & 4.018459 & 1.986912 \\
\hline $\mathrm{H}$ & -6.969820 & 1.117617 & 0.402214 \\
\hline $\mathrm{H}$ & -6.543130 & 3.208994 & 1.663739 \\
\hline $\mathrm{C}$ & -1.366297 & 1.180524 & -1.740163 \\
\hline $\mathrm{C}$ & -0.121321 & 2.720571 & -3.723821 \\
\hline C & -1.877833 & 2.429174 & -2.104343 \\
\hline C & -0.212490 & 0.692205 & -2.392449 \\
\hline $\mathrm{C}$ & 0.402752 & 1.484281 & -3.362622 \\
\hline $\mathrm{C}$ & -1.266794 & 3.190885 & -3.095746 \\
\hline $\mathrm{H}$ & -2.765286 & 2.817241 & -1.608315 \\
\hline $\mathrm{H}$ & 1.327841 & 1.137467 & -3.825608 \\
\hline $\mathrm{H}$ & -1.681744 & 4.160451 & -3.361805 \\
\hline $\mathrm{H}$ & 0.376434 & 3.319979 & -4.482947 \\
\hline C & 1.330569 & 2.845066 & -0.022654 \\
\hline C & 0.989553 & 5.532632 & -0.686980 \\
\hline C & 0.472880 & 3.655817 & 0.730376 \\
\hline $\mathrm{C}$ & 2.021584 & 3.391327 & -1.105025 \\
\hline C & 1.849297 & 4.733811 & -1.433809 \\
\hline $\mathrm{C}$ & 0.302607 & 4.994026 & 0.399922 \\
\hline $\mathrm{H}$ & -0.045113 & 3.236324 & 1.597366 \\
\hline $\mathrm{H}$ & 2.700855 & 2.771765 & -1.692156 \\
\hline $\mathrm{H}$ & 2.396464 & 5.158608 & -2.273606 \\
\hline $\mathrm{H}$ & -0.357037 & 5.622543 & 0.995171 \\
\hline $\mathrm{H}$ & 0.861684 & 6.582161 & -0.944065 \\
\hline $\mathrm{H}$ & -3.237714 & -0.133416 & -3.066895 \\
\hline $\mathrm{H}$ & 4.584358 & 0.771926 & -0.321183 \\
\hline C & 3.870407 & 0.286440 & -0.978993 \\
\hline C & 2.449515 & 0.277638 & -0.800746 \\
\hline $\mathrm{H}$ & 5.191424 & -0.764172 & -2.444626 \\
\hline $\mathrm{C}$ & 1.892197 & -0.561698 & -1.844621 \\
\hline C & 2.982326 & -1.045337 & -2.634283 \\
\hline $\mathrm{H}$ & 2.937808 & -1.732831 & -3.473655 \\
\hline C & 4.192349 & -0.527785 & -2.094247 \\
\hline $\mathrm{Fe}$ & 3.186720 & -1.597858 & -0.673810 \\
\hline $\mathrm{H}$ & 2.124965 & -1.881142 & 1.925706 \\
\hline C & 2.762385 & -2.353019 & 1.185663 \\
\hline C & 4.160758 & -2.134774 & 1.036824 \\
\hline $\mathrm{H}$ & 1.337060 & -3.640548 & 0.046308 \\
\hline $\mathrm{H}$ & 4.762270 & -1.449627 & 1.627060 \\
\hline C & 4.610450 & -2.923761 & -0.059984 \\
\hline $\mathrm{H}$ & 5.621108 & -2.950503 & -0.453342 \\
\hline C & 3.489779 & -3.621216 & -0.593658 \\
\hline
\end{tabular}

\begin{tabular}{rrrr}
$\mathrm{H}$ & 3.510536 & -4.293329 & -1.446102 \\
$\mathrm{C}$ & 2.347401 & -3.266982 & 0.180653 \\
$\mathrm{C}$ & 0.404796 & -0.642864 & -2.026736 \\
$\mathrm{H}$ & -0.014305 & -0.978520 & -1.064333 \\
$\mathrm{~N}$ & -0.103663 & -1.731114 & -2.989719 \\
$\mathrm{C}$ & 0.268433 & -3.109422 & -2.557174 \\
$\mathrm{H}$ & -0.119755 & -3.262918 & -1.545846 \\
$\mathrm{H}$ & 1.356229 & -3.211529 & -2.559166 \\
$\mathrm{H}$ & -0.193880 & -3.825349 & -3.242632 \\
$\mathrm{C}$ & 0.145143 & -1.520573 & -4.446901 \\
$\mathrm{H}$ & -0.248985 & -0.545288 & -4.740316 \\
$\mathrm{H}$ & -0.379231 & -2.310135 & -4.993125 \\
$\mathrm{H}$ & 1.215341 & -1.573156 & -4.654955 \\
$\mathrm{Pd}$ & -0.549640 & -0.043522 & 1.254712 \\
$\mathrm{H}$ & -1.130138 & -1.669417 & -2.877872 \\
$\mathrm{P}$ & 1.506021 & 1.111210 & 0.514519 \\
$\mathrm{C}$ & -1.888364 & -1.120557 & 2.299890 \\
$\mathrm{C}$ & -3.029117 & -0.717790 & 2.849365 \\
$\mathrm{H}$ & -3.578652 & 0.164789 & 2.516397 \\
$\mathrm{C}$ & -1.180300 & -2.384154 & 2.704072 \\
$\mathrm{H}$ & -0.826311 & -2.962718 & 1.833885 \\
$\mathrm{H}$ & -0.298846 & -2.174973 & 3.330196 \\
$\mathrm{C}$ & -2.180654 & -3.237884 & 3.509977 \\
$\mathrm{H}$ & -1.669431 & -4.073762 & 3.999020 \\
$\mathrm{H}$ & -2.945296 & -3.645670 & 2.820478 \\
$\mathrm{O}$ & -2.774206 & -2.468826 & 4.510505 \\
$\mathrm{C}$ & -3.630803 & -1.487955 & 4.000384 \\
$\mathrm{H}$ & -4.583914 & -1.938579 & 3.658172 \\
$\mathrm{H}$ & -3.870513 & -0.812082 & 4.830195 \\
\hline & -1060
\end{tabular}

SCF energy: -2918.232419140

M06-D3 (Gas Phase) /6-31G(d) +SDD (Pd, Fe) opt. No imaginary frequency 


\begin{tabular}{|c|c|c|c|}
\hline \multicolumn{4}{|c|}{ Int-4STania_conf 5} \\
\hline $\mathrm{P}$ & -2.159066 & 0.076861 & 0.401914 \\
\hline $\mathrm{C}$ & 2.525199 & -2.111445 & -1.189845 \\
\hline $\mathrm{C}$ & 4.134732 & -3.219616 & -3.182348 \\
\hline C & 2.196260 & -1.953404 & -2.539284 \\
\hline C & 3.647934 & -2.870834 & -0.844583 \\
\hline C & 4.453671 & -3.413012 & -1.839303 \\
\hline $\mathrm{C}$ & 2.997262 & -2.499409 & -3.536152 \\
\hline $\mathrm{H}$ & 1.289908 & -1.401619 & -2.819251 \\
\hline $\mathrm{H}$ & 3.881241 & -3.058308 & 0.204413 \\
\hline $\mathrm{H}$ & 5.327520 & -4.001298 & -1.567134 \\
\hline $\mathrm{H}$ & 2.731622 & -2.372467 & -4.583468 \\
\hline $\mathrm{H}$ & 4.765894 & -3.650374 & -3.956764 \\
\hline C & -2.677986 & 1.826274 & 0.532958 \\
\hline $\mathrm{C}$ & -3.458146 & 4.501735 & 0.778658 \\
\hline C & -3.111602 & 2.327685 & 1.773503 \\
\hline C & -2.665115 & 2.670832 & -0.580141 \\
\hline C & -3.056270 & 4.002397 & -0.457113 \\
\hline $\mathrm{C}$ & -3.492235 & 3.662946 & 1.893106 \\
\hline $\mathrm{H}$ & -2.364573 & 2.272905 & -1.548239 \\
\hline $\mathrm{H}$ & -3.049604 & 4.650616 & -1.331088 \\
\hline $\mathrm{H}$ & -3.837748 & 4.042595 & 2.852997 \\
\hline $\mathrm{H}$ & -3.762663 & 5.542009 & 0.872958 \\
\hline $\mathrm{C}$ & -3.669308 & -0.906062 & 0.269590 \\
\hline $\mathrm{C}$ & -5.940785 & -2.468907 & -0.111769 \\
\hline $\mathrm{C}$ & -4.931042 & -0.305223 & 0.233051 \\
\hline C & -3.547710 & -2.293031 & 0.099165 \\
\hline C & -4.683020 & -3.071006 & -0.080286 \\
\hline C & -6.063329 & -1.091706 & 0.043356 \\
\hline $\mathrm{H}$ & -5.036570 & 0.773190 & 0.342869 \\
\hline $\mathrm{H}$ & -2.560263 & -2.758821 & 0.105855 \\
\hline $\mathrm{H}$ & -4.588667 & -4.147231 & -0.207165 \\
\hline $\mathrm{H}$ & -7.045054 & -0.624188 & 0.014599 \\
\hline $\mathrm{H}$ & -6.828941 & -3.078617 & -0.263182 \\
\hline C & -1.421896 & -0.263158 & 2.050307 \\
\hline $\mathrm{C}$ & -0.233496 & -0.992849 & 4.482121 \\
\hline C & -2.010003 & -1.225243 & 2.875368 \\
\hline C & -0.220080 & 0.355890 & 2.463236 \\
\hline $\mathrm{C}$ & 0.362120 & -0.033961 & 3.669399 \\
\hline $\mathrm{C}$ & -1.423594 & -1.586134 & 4.084581 \\
\hline $\mathrm{H}$ & -2.935314 & -1.709450 & 2.569833 \\
\hline $\mathrm{H}$ & 1.319360 & 0.399563 & 3.963391 \\
\hline $\mathrm{H}$ & -1.895645 & -2.342968 & 4.707009 \\
\hline $\mathrm{H}$ & 0.244013 & -1.285807 & 5.414557 \\
\hline C & 1.210341 & -2.652146 & 1.307102 \\
\hline $\mathrm{C}$ & 0.815777 & -4.760025 & 3.091528 \\
\hline C & 0.268407 & -3.648421 & 1.026506 \\
\hline $\mathrm{C}$ & 1.955874 & -2.721682 & 2.485609 \\
\hline C & 1.757529 & -3.775316 & 3.373725 \\
\hline $\mathrm{C}$ & 0.072183 & -4.698983 & 1.914310 \\
\hline $\mathrm{H}$ & -0.295771 & -3.609932 & 0.090721 \\
\hline $\mathrm{H}$ & 2.698555 & -1.955200 & 2.711291 \\
\hline $\mathrm{H}$ & 2.347847 & -3.830967 & 4.286658 \\
\hline $\mathrm{H}$ & -0.653692 & -5.476870 & 1.685782 \\
\hline $\mathrm{H}$ & 0.667501 & -5.585155 & 3.785183 \\
\hline $\mathrm{H}$ & -3.163330 & 1.668865 & 2.642800 \\
\hline $\mathrm{H}$ & 4.552040 & -0.810819 & 0.607405 \\
\hline C & 3.876356 & -0.064419 & 1.012928 \\
\hline C & 2.451212 & -0.069829 & 0.869508 \\
\hline $\mathrm{H}$ & 5.276814 & 1.442637 & 1.887972 \\
\hline C & 1.955009 & 1.151350 & 1.474790 \\
\hline C & 3.085581 & 1.872510 & 1.973760 \\
\hline $\mathrm{H}$ & 3.092374 & 2.848956 & 2.448529 \\
\hline C & 4.260176 & 1.125079 & 1.682165 \\
\hline $\mathrm{Fe}$ & 3.245009 & 1.549321 & -0.039103 \\
\hline $\mathrm{H}$ & 2.239663 & 0.667308 & -2.523308 \\
\hline C & 2.847584 & 1.429455 & -2.047939 \\
\hline C & 4.246473 & 1.337278 & -1.806093 \\
\hline $\mathrm{H}$ & 1.363698 & 3.027910 & -1.568693 \\
\hline $\mathrm{H}$ & 4.877698 & 0.486435 & -2.044986 \\
\hline C & 4.654362 & 2.529234 & -1.142850 \\
\hline $\mathrm{H}$ & 5.655659 & 2.751162 & -0.789434 \\
\hline C & 3.506584 & 3.353283 & -0.967239 \\
\hline
\end{tabular}

\begin{tabular}{|c|c|c|c|}
\hline $\mathrm{H}$ & 3.496859 & 4.326080 & -0.484962 \\
\hline $\mathrm{C}$ & 2.388489 & 2.669464 & -1.527846 \\
\hline $\mathrm{C}$ & 0.477880 & 1.389366 & 1.602102 \\
\hline $\mathrm{H}$ & 0.060353 & 1.344238 & 0.583408 \\
\hline $\mathrm{N}$ & 0.062518 & 2.800983 & 2.055463 \\
\hline $\mathrm{C}$ & 0.490912 & 3.870777 & 1.107135 \\
\hline $\mathrm{H}$ & 0.070567 & 3.641133 & 0.123599 \\
\hline $\mathrm{H}$ & 1.581525 & 3.894210 & 1.051440 \\
\hline $\mathrm{H}$ & 0.096033 & 4.826888 & 1.462098 \\
\hline $\mathrm{C}$ & 0.348862 & 3.168619 & 3.474397 \\
\hline $\mathrm{H}$ & -0.089960 & 2.417355 & 4.134233 \\
\hline $\mathrm{H}$ & -0.110369 & 4.142482 & 3.667174 \\
\hline $\mathrm{H}$ & 1.426587 & 3.228354 & 3.635646 \\
\hline $\mathrm{Pd}$ & -0.551758 & -0.499581 & -1.148943 \\
\hline $\mathrm{H}$ & -0.968642 & 2.767167 & 1.984480 \\
\hline $\mathrm{P}$ & 1.437397 & -1.344545 & 0.055145 \\
\hline $\mathrm{C}$ & -1.815591 & 0.102282 & -2.594384 \\
\hline $\mathrm{C}$ & -2.986221 & -0.422390 & -2.940595 \\
\hline $\mathrm{H}$ & -3.595224 & -1.031054 & -2.269734 \\
\hline $\mathrm{C}$ & -1.024597 & 1.012457 & -3.493277 \\
\hline $\mathrm{H}$ & -0.164184 & 0.493542 & -3.943797 \\
\hline $\mathrm{H}$ & -0.625394 & 1.888979 & -2.955722 \\
\hline $\mathrm{C}$ & -1.968156 & 1.491347 & -4.615361 \\
\hline $\mathrm{H}$ & -2.698121 & 2.208787 & -4.192505 \\
\hline $\mathrm{H}$ & -1.402835 & 1.993642 & -5.407389 \\
\hline O & -2.620306 & 0.402829 & -5.193815 \\
\hline $\mathrm{C}$ & -3.539932 & -0.196003 & -4.327063 \\
\hline $\mathrm{H}$ & -3.832832 & -1.149548 & -4.782963 \\
\hline $\mathrm{H}$ & -4.456449 & 0.421196 & -4.239536 \\
\hline
\end{tabular}

SCF energy: -2918.232301120

M06-D3 (Gas Phase) /6-31G(d) +SDD (Pd, Fe) opt. No imaginary frequency 


\begin{tabular}{|c|c|c|c|}
\hline \multicolumn{4}{|c|}{ Int-4RTania_conf1 } \\
\hline $\mathrm{P}$ & -2.201987 & -0.320736 & 0.627643 \\
\hline $\mathrm{C}$ & 2.465515 & -1.010922 & -1.959045 \\
\hline $\mathrm{C}$ & 4.097773 & -0.996646 & -4.223420 \\
\hline C & 2.113478 & -0.257316 & -3.082302 \\
\hline C & 3.622594 & -1.797376 & -1.997775 \\
\hline C & 4.439558 & -1.779642 & -3.122055 \\
\hline $\mathrm{C}$ & 2.927151 & -0.244266 & -4.210251 \\
\hline $\mathrm{H}$ & 1.183909 & 0.321154 & -3.082303 \\
\hline $\mathrm{H}$ & 3.875734 & -2.442561 & -1.155400 \\
\hline $\mathrm{H}$ & 5.339893 & -2.390063 & -3.146261 \\
\hline $\mathrm{H}$ & 2.643872 & 0.343593 & -5.080815 \\
\hline $\mathrm{H}$ & 4.737317 & -0.990180 & -5.103548 \\
\hline C & -2.824793 & 1.151746 & 1.518030 \\
\hline $\mathrm{C}$ & -3.744179 & 3.385701 & 2.927298 \\
\hline C & -3.286761 & 1.015748 & 2.839508 \\
\hline C & -2.839628 & 2.411310 & 0.911365 \\
\hline C & -3.304758 & 3.521967 & 1.613291 \\
\hline $\mathrm{C}$ & -3.737866 & 2.132079 & 3.540384 \\
\hline $\mathrm{H}$ & -2.486658 & 2.518550 & -0.116748 \\
\hline $\mathrm{H}$ & -3.321688 & 4.497421 & 1.131276 \\
\hline $\mathrm{H}$ & -4.103739 & 2.019651 & 4.559325 \\
\hline $\mathrm{H}$ & -4.104549 & 4.254807 & 3.473825 \\
\hline $\mathrm{C}$ & -3.648707 & -1.276021 & 0.111135 \\
\hline $\mathrm{C}$ & -5.823577 & -2.703622 & -0.879487 \\
\hline $\mathrm{C}$ & -4.950082 & -0.861407 & 0.409956 \\
\hline C & -3.440314 & -2.407908 & -0.689668 \\
\hline C & -4.526435 & -3.122915 & -1.174156 \\
\hline C & -6.033983 & -1.578533 & -0.089127 \\
\hline $\mathrm{H}$ & -5.125722 & 0.024391 & 1.019138 \\
\hline $\mathrm{H}$ & -2.424115 & -2.728289 & -0.929343 \\
\hline $\mathrm{H}$ & -4.364117 & -4.003838 & -1.791461 \\
\hline $\mathrm{H}$ & -7.046388 & -1.253343 & 0.140052 \\
\hline $\mathrm{H}$ & -6.673878 & -3.258056 & -1.270733 \\
\hline C & -1.355856 & -1.277316 & 1.945628 \\
\hline $\mathrm{C}$ & 0.001307 & -2.858290 & 3.817964 \\
\hline C & -1.827480 & -2.548495 & 2.284167 \\
\hline C & -0.186833 & -0.787039 & 2.566675 \\
\hline $\mathrm{C}$ & 0.486727 & -1.599928 & 3.479153 \\
\hline $\mathrm{C}$ & -1.161636 & -3.329872 & 3.223242 \\
\hline $\mathrm{H}$ & -2.724374 & -2.939035 & 1.807050 \\
\hline $\mathrm{H}$ & 1.424208 & -1.250677 & 3.914496 \\
\hline $\mathrm{H}$ & -1.546458 & -4.315972 & 3.473304 \\
\hline $\mathrm{H}$ & 0.542427 & -3.473566 & 4.533497 \\
\hline C & 1.229508 & -2.732942 & -0.033604 \\
\hline $\mathrm{C}$ & 0.939725 & -5.454806 & 0.504924 \\
\hline C & 0.351685 & -3.514525 & -0.794196 \\
\hline $\mathrm{C}$ & 1.967883 & -3.325922 & 0.991274 \\
\hline C & 1.821253 & -4.685335 & 1.257093 \\
\hline C & 0.205675 & -4.869396 & -0.525561 \\
\hline $\mathrm{H}$ & -0.200463 & -3.057816 & -1.620464 \\
\hline $\mathrm{H}$ & 2.666414 & -2.730252 & 1.580571 \\
\hline $\mathrm{H}$ & 2.405605 & -5.146154 & 2.051657 \\
\hline $\mathrm{H}$ & -0.470404 & -5.474318 & -1.126920 \\
\hline $\mathrm{H}$ & 0.831980 & -6.517431 & 0.712681 \\
\hline $\mathrm{H}$ & -3.301547 & 0.033196 & 3.315591 \\
\hline $\mathrm{H}$ & 4.482101 & -0.589690 & 0.185271 \\
\hline C & 3.796394 & -0.172828 & 0.915943 \\
\hline C & 2.366952 & -0.200199 & 0.822760 \\
\hline $\mathrm{H}$ & 5.173200 & 0.822440 & 2.368515 \\
\hline $\mathrm{C}$ & 1.850952 & 0.549579 & 1.952943 \\
\hline C & 2.972827 & 1.016418 & 2.707018 \\
\hline $\mathrm{H}$ & 2.959820 & 1.644502 & 3.592798 \\
\hline C & 4.161907 & 0.576702 & 2.062480 \\
\hline $\mathrm{Fe}$ & 3.038448 & 1.702376 & 0.778433 \\
\hline $\mathrm{H}$ & 2.004567 & 2.000961 & -1.825369 \\
\hline C & 2.573745 & 2.503230 & -1.050844 \\
\hline C & 3.983746 & 2.420975 & -0.882707 \\
\hline $\mathrm{H}$ & 1.000151 & 3.568906 & 0.121654 \\
\hline $\mathrm{H}$ & 4.661313 & 1.830535 & -1.492662 \\
\hline C & 4.332518 & 3.193420 & 0.261260 \\
\hline $\mathrm{H}$ & 5.328669 & 3.303861 & 0.676256 \\
\hline C & 3.137413 & 3.745542 & 0.803969 \\
\hline
\end{tabular}

\begin{tabular}{rrrr}
$\mathrm{H}$ & 3.079273 & 4.378444 & 1.684298 \\
$\mathrm{C}$ & 2.048586 & 3.315468 & -0.009843 \\
$\mathrm{C}$ & 0.376520 & 0.578020 & 2.227052 \\
$\mathrm{H}$ & -0.106998 & 0.946039 & 1.308546 \\
$\mathrm{~N}$ & -0.100525 & 1.606629 & 3.271156 \\
$\mathrm{C}$ & 0.236346 & 3.013290 & 2.904100 \\
$\mathrm{H}$ & -0.182823 & 3.218998 & 1.915818 \\
$\mathrm{H}$ & 1.321793 & 3.132073 & 2.880547 \\
$\mathrm{H}$ & -0.215050 & 3.681215 & 3.643067 \\
$\mathrm{C}$ & 0.224525 & 1.323361 & 4.701347 \\
$\mathrm{H}$ & -0.154577 & 0.335054 & 4.968164 \\
$\mathrm{H}$ & -0.266857 & 2.084996 & 5.313451 \\
$\mathrm{H}$ & 1.304697 & 1.366744 & 4.852940 \\
$\mathrm{Pd}$ & -0.744990 & 0.122380 & -1.105749 \\
$\mathrm{H}$ & -1.130255 & 1.536100 & 3.202944 \\
$\mathrm{P}$ & 1.376160 & -0.974220 & -0.495792 \\
$\mathrm{C}$ & -2.160233 & 1.082306 & -2.162737 \\
$\mathrm{C}$ & -1.482368 & 2.102633 & -2.696334 \\
$\mathrm{H}$ & -0.585010 & 2.541921 & -2.243350 \\
$\mathrm{C}$ & -3.484936 & 0.623168 & -2.648731 \\
$\mathrm{H}$ & -4.217685 & 0.570137 & -1.826388 \\
$\mathrm{H}$ & -3.414793 & -0.393391 & -3.063828 \\
$\mathrm{C}$ & -3.978691 & 1.624864 & -3.703718 \\
$\mathrm{H}$ & -4.802524 & 1.202531 & -4.287400 \\
$\mathrm{H}$ & -4.337861 & 2.542144 & -3.201417 \\
$\mathrm{O}$ & -2.949653 & 1.936114 & -4.606833 \\
$\mathrm{C}$ & -1.940708 & 2.693325 & -4.017415 \\
$\mathrm{H}$ & -2.278218 & 3.733091 & -3.832961 \\
$\mathrm{H}$ & -1.108484 & 2.738443 & -4.730767 \\
\hline & - & $-100-1906$
\end{tabular}

SCF energy: -2918.234164380

M06-D3 (Gas Phase) /6-31G(d)+SDD (Pd, Fe) opt. No imaginary frequency 


\begin{tabular}{|c|c|c|c|}
\hline \multicolumn{4}{|c|}{ Int-5STania_conf 7} \\
\hline $\mathrm{P}$ & 0.296914 & -1.534691 & 0.730011 \\
\hline $\mathrm{P}$ & 0.332365 & 2.213773 & 0.292400 \\
\hline $\mathrm{C}$ & -0.565912 & -3.148467 & 0.532231 \\
\hline C & -1.955543 & -5.561833 & 0.208679 \\
\hline C & -0.928494 & -3.600027 & -0.741674 \\
\hline $\mathrm{C}$ & -0.914788 & -3.921188 & 1.644026 \\
\hline $\mathrm{C}$ & -1.606296 & -5.119487 & 1.479930 \\
\hline $\mathrm{C}$ & -1.610199 & -4.799877 & -0.906039 \\
\hline $\mathrm{H}$ & -0.688024 & -3.007101 & -1.622123 \\
\hline $\mathrm{H}$ & -0.656276 & -3.592061 & 2.649227 \\
\hline $\mathrm{H}$ & -1.871644 & -5.708748 & 2.355409 \\
\hline $\mathrm{H}$ & -1.884246 & -5.133769 & -1.905574 \\
\hline $\mathrm{H}$ & -2.496993 & -6.497359 & 0.084356 \\
\hline $\mathrm{C}$ & 1.287441 & 3.277756 & -0.839130 \\
\hline C & 2.811910 & 4.859644 & -2.563507 \\
\hline C & 1.813869 & 4.498088 & -0.395067 \\
\hline C & 1.543708 & 2.852956 & -2.145345 \\
\hline C & 2.306188 & 3.639780 & -3.005953 \\
\hline $\mathrm{C}$ & 2.565236 & 5.288210 & -1.259121 \\
\hline $\mathrm{H}$ & 1.133478 & 1.900587 & -2.486717 \\
\hline $\mathrm{H}$ & 2.486729 & 3.310273 & -4.027994 \\
\hline $\mathrm{H}$ & 2.957842 & 6.243103 & -0.915575 \\
\hline $\mathrm{H}$ & 3.394235 & 5.483904 & -3.238068 \\
\hline $\mathrm{C}$ & -0.971519 & 3.244717 & 1.031343 \\
\hline $\mathrm{C}$ & -3.199301 & 4.614318 & 2.013050 \\
\hline C & -1.312205 & 4.496136 & 0.511373 \\
\hline C & -1.771014 & 2.673182 & 2.031241 \\
\hline C & -2.872244 & 3.360234 & 2.525746 \\
\hline C & -2.420376 & 5.177661 & 1.007362 \\
\hline $\mathrm{H}$ & -0.733830 & 4.935758 & -0.299458 \\
\hline $\mathrm{H}$ & -1.528614 & 1.686470 & 2.429280 \\
\hline $\mathrm{H}$ & -3.479057 & 2.913324 & 3.311577 \\
\hline $\mathrm{H}$ & -2.677207 & 6.152604 & 0.598530 \\
\hline $\mathrm{H}$ & -4.064718 & 5.150811 & 2.396171 \\
\hline $\mathrm{C}$ & 1.525895 & 1.859704 & 1.670140 \\
\hline $\mathrm{C}$ & 3.169649 & 1.230282 & 3.872225 \\
\hline $\mathrm{C}$ & 1.293738 & 2.412771 & 2.935202 \\
\hline $\mathrm{C}$ & 2.630437 & 0.997083 & 1.516169 \\
\hline $\mathrm{C}$ & 3.417018 & 0.674972 & 2.624012 \\
\hline $\mathrm{C}$ & 2.109479 & 2.115294 & 4.021331 \\
\hline $\mathrm{H}$ & 0.457091 & 3.091546 & 3.082165 \\
\hline $\mathrm{H}$ & 4.238470 & -0.036749 & 2.510490 \\
\hline $\mathrm{H}$ & 1.902217 & 2.568348 & 4.988255 \\
\hline $\mathrm{H}$ & 3.797169 & 0.968722 & 4.721420 \\
\hline $\mathrm{C}$ & 0.152094 & -1.249605 & 2.528281 \\
\hline $\mathrm{C}$ & -0.280274 & -0.899637 & 5.264515 \\
\hline $\mathrm{C}$ & -1.070570 & -0.750300 & 2.993170 \\
\hline $\mathrm{C}$ & 1.148168 & -1.590644 & 3.443773 \\
\hline $\mathrm{C}$ & 0.930090 & -1.412737 & 4.808230 \\
\hline $\mathrm{C}$ & -1.284014 & -0.572594 & 4.354523 \\
\hline $\mathrm{H}$ & -1.865251 & -0.538653 & 2.275610 \\
\hline $\mathrm{H}$ & 2.092571 & -2.011607 & 3.095771 \\
\hline $\mathrm{H}$ & 1.708237 & -1.686002 & 5.519001 \\
\hline $\mathrm{H}$ & -2.241534 & -0.189315 & 4.707087 \\
\hline $\mathrm{H}$ & -0.447501 & -0.768173 & 6.331656 \\
\hline $\mathrm{H}$ & 1.634070 & 4.832894 & 0.627865 \\
\hline $\mathrm{H}$ & 1.969413 & -4.187443 & 0.612938 \\
\hline $\mathrm{C}$ & 2.567606 & -3.290565 & 0.482021 \\
\hline $\mathrm{C}$ & 2.047076 & -1.957685 & 0.461477 \\
\hline $\mathrm{H}$ & 4.624187 & -4.094014 & 0.132077 \\
\hline $\mathrm{C}$ & 3.153272 & -1.067315 & 0.178835 \\
\hline $\mathrm{C}$ & 4.330397 & -1.880759 & 0.056654 \\
\hline $\mathrm{H}$ & 5.341332 & -1.560649 & -0.187262 \\
\hline $\mathrm{C}$ & 3.960081 & -3.241989 & 0.229681 \\
\hline $\mathrm{Fe}$ & 2.918950 & -2.393944 & -1.320812 \\
\hline $\mathrm{H}$ & 0.535440 & -1.759700 & -2.682181 \\
\hline $\mathrm{C}$ & 1.527667 & -2.184524 & -2.809024 \\
\hline $\mathrm{C}$ & 1.901793 & -3.551500 & -2.659577 \\
\hline $\mathrm{H}$ & 2.723203 & -0.368896 & -3.308362 \\
\hline $\mathrm{H}$ & 1.247869 & -4.368306 & -2.370975 \\
\hline $\mathrm{C}$ & 3.302749 & -3.647780 & -2.889068 \\
\hline $\mathrm{H}$ & 3.899798 & -4.550130 & -2.812636 \\
\hline
\end{tabular}

\begin{tabular}{|c|c|c|c|}
\hline C & 3.795028 & -2.343711 & -3.175001 \\
\hline $\mathrm{H}$ & 4.831420 & -2.092879 & -3.380893 \\
\hline C & 2.693877 & -1.439851 & -3.127176 \\
\hline C & 3.059638 & 0.438579 & 0.180345 \\
\hline $\mathrm{H}$ & 2.392347 & 0.781492 & -0.622358 \\
\hline $\mathrm{N}$ & 4.438750 & 1.039875 & -0.191933 \\
\hline C & 4.622658 & 2.487060 & 0.151359 \\
\hline $\mathrm{H}$ & 4.481714 & 2.640380 & 1.222254 \\
\hline $\mathrm{H}$ & 3.893919 & 3.073981 & -0.413443 \\
\hline $\mathrm{H}$ & 5.637683 & 2.770315 & -0.139925 \\
\hline C & 4.773442 & 0.843136 & -1.636781 \\
\hline $\mathrm{H}$ & 4.593298 & -0.198241 & -1.909698 \\
\hline $\mathrm{H}$ & 5.820382 & 1.115802 & -1.795880 \\
\hline $\mathrm{H}$ & 4.125065 & 1.510059 & -2.215378 \\
\hline $\mathrm{Pd}$ & -0.638862 & 0.317960 & -0.632857 \\
\hline C & -1.676886 & 1.620806 & -1.837427 \\
\hline C & -2.744472 & 2.264766 & -1.375587 \\
\hline $\mathrm{H}$ & -2.970152 & 2.341850 & -0.310215 \\
\hline $\mathrm{C}$ & -1.436458 & 1.438963 & -3.307183 \\
\hline $\mathrm{H}$ & -0.692356 & 2.160171 & -3.678418 \\
\hline $\mathrm{H}$ & -1.052854 & 0.432308 & -3.544811 \\
\hline $\mathrm{C}$ & -2.767499 & 1.666294 & -4.032977 \\
\hline $\mathrm{H}$ & -3.464375 & 0.838881 & -3.804046 \\
\hline $\mathrm{H}$ & -2.620898 & 1.709465 & -5.117693 \\
\hline 0 & -3.313497 & 2.899202 & -3.642257 \\
\hline $\mathrm{C}$ & -3.745911 & 2.886736 & -2.313633 \\
\hline $\mathrm{H}$ & -3.945079 & 3.929176 & -2.029601 \\
\hline $\mathrm{H}$ & -4.702862 & 2.331688 & -2.215550 \\
\hline 0 & -3.002942 & -1.147357 & 0.342221 \\
\hline B & -2.865011 & -1.018608 & -0.998285 \\
\hline O & -3.995272 & -1.082584 & -1.723529 \\
\hline $\mathrm{C}$ & -5.020075 & -1.579790 & -0.797677 \\
\hline C & -4.441499 & -1.175677 & 0.599120 \\
\hline C & -4.687294 & -2.172869 & 1.710082 \\
\hline $\mathrm{H}$ & -4.231054 & -3.144809 & 1.488861 \\
\hline $\mathrm{H}$ & -5.763215 & -2.315472 & 1.877162 \\
\hline $\mathrm{H}$ & -4.248442 & -1.801950 & 2.645940 \\
\hline C & -4.826118 & 0.226644 & 1.036536 \\
\hline $\mathrm{H}$ & -4.655040 & 0.962115 & 0.239006 \\
\hline $\mathrm{H}$ & -4.211411 & 0.514786 & 1.901564 \\
\hline $\mathrm{H}$ & -5.879927 & 0.279710 & 1.335736 \\
\hline $\mathrm{C}$ & -5.080309 & -3.081825 & -1.009621 \\
\hline $\mathrm{H}$ & -4.124216 & -3.563831 & -0.761561 \\
\hline $\mathrm{H}$ & -5.300742 & -3.284220 & -2.064112 \\
\hline $\mathrm{H}$ & -5.869083 & -3.541309 & -0.401724 \\
\hline $\mathrm{C}$ & -6.341119 & -0.936472 & -1.150155 \\
\hline $\mathrm{H}$ & -6.280293 & 0.156940 & -1.159894 \\
\hline $\mathrm{H}$ & -7.116240 & -1.236833 & -0.432160 \\
\hline $\mathrm{H}$ & -6.660294 & -1.266566 & -2.145367 \\
\hline $\mathrm{H}$ & -1.766776 & -0.972908 & -1.550574 \\
\hline $\mathrm{H}$ & 5.121808 & 0.511782 & 0.364777 \\
\hline
\end{tabular}

SCF energy: -3329.876874400

M06-D3 (Gas Phase) /6-31G(d)+SDD (Pd, Fe) opt. No imaginary frequency 


\begin{tabular}{|c|c|c|c|}
\hline \multicolumn{4}{|c|}{ Int-5RTania_conf3 } \\
\hline $\mathrm{P}$ & 0.267653 & 2.307807 & 0.439088 \\
\hline $\mathrm{C}$ & -0.524480 & -3.121861 & 0.274501 \\
\hline $\mathrm{C}$ & -1.866335 & -5.517340 & -0.307254 \\
\hline $\mathrm{C}$ & -0.941365 & -3.413541 & -1.030212 \\
\hline $\mathrm{C}$ & -0.790372 & -4.053862 & 1.285010 \\
\hline $\mathrm{C}$ & -1.451114 & -5.243733 & 0.992176 \\
\hline $\mathrm{C}$ & -1.608965 & -4.598081 & -1.321113 \\
\hline $\mathrm{H}$ & -0.755020 & -2.709000 & -1.837533 \\
\hline $\mathrm{H}$ & -0.495008 & -3.853251 & 2.313574 \\
\hline $\mathrm{H}$ & -1.649185 & -5.956086 & 1.790524 \\
\hline $\mathrm{H}$ & -1.934715 & -4.796856 & -2.340321 \\
\hline $\mathrm{H}$ & -2.391379 & -6.443911 & -0.529939 \\
\hline $\mathrm{C}$ & 1.414963 & 3.447629 & -0.425576 \\
\hline C & 3.216326 & 5.169094 & -1.701840 \\
\hline $\mathrm{C}$ & 2.316317 & 4.220380 & 0.329133 \\
\hline C & 1.416546 & 3.561679 & -1.818417 \\
\hline C & 2. 311856 & 4.422948 & -2.451099 \\
\hline $\mathrm{C}$ & 3.215682 & 5.071191 & -0.309746 \\
\hline $\mathrm{H}$ & 0.699692 & 2.986017 & -2.402825 \\
\hline $\mathrm{H}$ & 2.297476 & 4.512500 & -3.535554 \\
\hline $\mathrm{H}$ & 3.901834 & 5.674908 & 0.281564 \\
\hline $\mathrm{H}$ & 3.913070 & 5.841263 & -2.198516 \\
\hline C & -1.062623 & 3.334721 & 1.121035 \\
\hline $\mathrm{C}$ & -3.218058 & 4.820433 & 2.073071 \\
\hline C & -1.078301 & 4.722988 & 0.962565 \\
\hline $\mathrm{C}$ & -2.135593 & 2.691514 & 1.753799 \\
\hline C & -3.202219 & 3.436320 & 2.238020 \\
\hline $\mathrm{C}$ & -2.159836 & 5.460598 & 1.436125 \\
\hline $\mathrm{H}$ & -0.259814 & 5.233890 & 0.457490 \\
\hline $\mathrm{H}$ & -2.143699 & 1.603781 & 1.852047 \\
\hline $\mathrm{H}$ & -4.030274 & 2.935926 & 2.736697 \\
\hline $\mathrm{H}$ & -2.173852 & 6.540308 & 1.304626 \\
\hline $\mathrm{H}$ & -4.061154 & 5.401471 & 2.440815 \\
\hline C & 1.289199 & 1.773998 & 1.874629 \\
\hline $\mathrm{C}$ & 2.721760 & 0.843049 & 4.096598 \\
\hline C & 0.906162 & 2.125274 & 3.170637 \\
\hline $\mathrm{C}$ & 2.420879 & 0.952338 & 1.692477 \\
\hline $\mathrm{C}$ & 3.104510 & 0.476031 & 2.811128 \\
\hline $\mathrm{C}$ & 1.627235 & 1.678876 & 4.274418 \\
\hline $\mathrm{H}$ & 0.031858 & 2.754989 & 3.325495 \\
\hline $\mathrm{H}$ & 3.936111 & -0.217199 & 2.674671 \\
\hline $\mathrm{H}$ & 1.313718 & 1.967923 & 5.275170 \\
\hline $\mathrm{H}$ & 3.269043 & 0.461596 & 4.955883 \\
\hline C & 0.149104 & -1.451515 & 2.470198 \\
\hline $\mathrm{C}$ & -0.341435 & -1.541363 & 5.217962 \\
\hline $\mathrm{C}$ & -1.033746 & -0.919536 & 2.994856 \\
\hline $\mathrm{C}$ & 1.084523 & -2.028494 & 3.332521 \\
\hline $\mathrm{C}$ & 0.837565 & -2.072415 & 4.701931 \\
\hline C & -1.277783 & -0.964575 & 4.363020 \\
\hline $\mathrm{H}$ & -1.783208 & -0.508960 & 2.315448 \\
\hline $\mathrm{H}$ & 2.001746 & -2.464529 & 2.933134 \\
\hline $\mathrm{H}$ & 1.564938 & -2.533932 & 5.367548 \\
\hline $\mathrm{H}$ & -2.205662 & -0.557229 & 4.762315 \\
\hline $\mathrm{H}$ & -0.535129 & -1.586498 & 6.287840 \\
\hline $\mathrm{H}$ & 2.305925 & 4.165409 & 1.419485 \\
\hline $\mathrm{H}$ & 2.078890 & -4.103304 & 0.248748 \\
\hline C & 2.638171 & -3.173189 & 0.208324 \\
\hline $\mathrm{C}$ & 2.067342 & -1.867067 & 0.336663 \\
\hline $\mathrm{H}$ & 4.717788 & -3.848203 & -0.258714 \\
\hline $\mathrm{C}$ & 3.139857 & -0.908340 & 0.146192 \\
\hline C & 4.340030 & -1.652099 & -0.088710 \\
\hline $\mathrm{H}$ & 5.328376 & -1.261396 & -0.311421 \\
\hline C & 4.023545 & -3.038905 & -0.059068 \\
\hline $\mathrm{Fe}$ & 2.920021 & -2.082909 & -1.490186 \\
\hline $\mathrm{H}$ & 0.489494 & -1.431370 & -2.774394 \\
\hline $\mathrm{C}$ & 1.499915 & -1.796153 & -2.941044 \\
\hline $\mathrm{C}$ & 1.935275 & -3.152658 & -2.922653 \\
\hline $\mathrm{H}$ & 2.607067 & 0.108379 & -3.280809 \\
\hline $\mathrm{H}$ & 1.320048 & -4.020902 & -2.707843 \\
\hline C & 3.336633 & -3.162889 & -3.171940 \\
\hline $\mathrm{H}$ & 3.973690 & -4.040797 & -3.184237 \\
\hline C & 3.768541 & -1.816621 & -3.335731 \\
\hline
\end{tabular}

\begin{tabular}{|c|c|c|c|}
\hline $\mathrm{H}$ & 4.789909 & -1.499047 & -3.522259 \\
\hline $\mathrm{C}$ & 2.628850 & -0.973662 & -3.194459 \\
\hline $\mathrm{C}$ & 2.889617 & 0.563757 & 0.306844 \\
\hline $\mathrm{H}$ & 2.110383 & 0.833056 & -0.422968 \\
\hline $\mathrm{N}$ & 4.048878 & 1.497380 & -0.104308 \\
\hline $\mathrm{C}$ & 4.405895 & 1.401298 & -1.550251 \\
\hline $\mathrm{H}$ & 3.510846 & 1.625423 & -2.135999 \\
\hline $\mathrm{H}$ & 4.761463 & 0.393059 & -1.773377 \\
\hline $\mathrm{H}$ & 5.178506 & 2.144716 & -1.765485 \\
\hline $\mathrm{C}$ & 5.268070 & 1.488465 & 0.759254 \\
\hline $\mathrm{H}$ & 4.983953 & 1.723044 & 1.786399 \\
\hline $\mathrm{H}$ & 5.950596 & 2.255178 & 0.382412 \\
\hline $\mathrm{H}$ & 5.748880 & 0.509689 & 0.714932 \\
\hline $\mathrm{Pd}$ & -0.630787 & 0.444217 & -0.624914 \\
\hline $\mathrm{H}$ & 3.630456 & 2.436538 & 0.013815 \\
\hline $\mathrm{P}$ & 0.303961 & -1.517527 & 0.650156 \\
\hline $\mathrm{C}$ & -1.543784 & 1.744275 & -1.922104 \\
\hline C & -1.149095 & 1.615772 & -3.187413 \\
\hline $\mathrm{H}$ & -0.262114 & 1.043988 & -3.478586 \\
\hline $\mathrm{C}$ & -2.798808 & 2.457987 & -1.540216 \\
\hline $\mathrm{H}$ & -3.336482 & 1.928798 & -0.735706 \\
\hline $\mathrm{H}$ & -2.585315 & 3.467915 & -1.156109 \\
\hline C & -3.680214 & 2.548940 & -2.790097 \\
\hline $\mathrm{H}$ & -4.538174 & 3.208776 & -2.619142 \\
\hline $\mathrm{H}$ & -4.059839 & 1.541671 & -3.050891 \\
\hline $\mathrm{O}$ & -2.946551 & 3.089588 & -3.856764 \\
\hline $\mathrm{C}$ & -1.964051 & 2.209800 & -4.316718 \\
\hline $\mathrm{H}$ & -2.414920 & 1.383800 & -4.901705 \\
\hline $\mathrm{H}$ & -1.322462 & 2.779471 & -5.001801 \\
\hline O & -3.160865 & -0.646789 & 0.397934 \\
\hline B & -2.939925 & -0.984677 & -0.897499 \\
\hline O & -3.979103 & -1.515709 & -1.559880 \\
\hline C & -5.132487 & -1.397261 & -0.664494 \\
\hline C & -4.460025 & -1.234645 & 0.739650 \\
\hline C & -4.162439 & -2.555432 & 1.426445 \\
\hline $\mathrm{H}$ & -3.633588 & -3.248913 & 0.759575 \\
\hline $\mathrm{H}$ & -5.082697 & -3.040816 & 1.773035 \\
\hline $\mathrm{H}$ & -3.522288 & -2.376903 & 2.300780 \\
\hline C & -5.160709 & -0.285388 & 1.685287 \\
\hline $\mathrm{H}$ & -5.240135 & 0.725033 & 1.267223 \\
\hline $\mathrm{H}$ & -4.599253 & -0.225752 & 2.628246 \\
\hline $\mathrm{H}$ & -6.170775 & -0.644619 & 1.922704 \\
\hline $\mathrm{C}$ & -5.980132 & -2.639336 & -0.820916 \\
\hline $\mathrm{H}$ & -5.393958 & -3.552926 & -0.674705 \\
\hline $\mathrm{H}$ & -6.408878 & -2.670069 & -1.829175 \\
\hline $\mathrm{H}$ & -6.810311 & -2.633376 & -0.101798 \\
\hline $\mathrm{C}$ & -5.897527 & -0.167029 & -1.117890 \\
\hline $\mathrm{H}$ & -5.298734 & 0.748787 & -1.012119 \\
\hline $\mathrm{H}$ & -6.826125 & -0.039097 & -0.548343 \\
\hline $\mathrm{H}$ & -6.160143 & -0.279225 & -2.176479 \\
\hline $\mathrm{H}$ & -1.871228 & -0.832198 & -1.465338 \\
\hline
\end{tabular}

SCF energy: -3329.873892000

M06-D3 (Gas Phase) /6-31G(d)+SDD (Pd, Fe) opt. No imaginary frequency 


\begin{tabular}{|c|c|c|c|}
\hline \multicolumn{4}{|c|}{ Int-5RTania_conf2 } \\
\hline $\mathrm{P}$ & 0.275545 & -1.495219 & 0.719195 \\
\hline $\mathrm{P}$ & 0.401867 & 2.257193 & 0.284121 \\
\hline $\mathrm{C}$ & -0.594029 & -3.099419 & 0.471096 \\
\hline $\mathrm{C}$ & -1.985448 & -5.498381 & 0.061901 \\
\hline $\mathrm{C}$ & -0.952415 & -3.507268 & -0.818827 \\
\hline $\mathrm{C}$ & -0.949572 & -3.906445 & 1.555336 \\
\hline $\mathrm{C}$ & -1.641192 & -5.098341 & 1.348442 \\
\hline $\mathrm{C}$ & -1.636313 & -4.699553 & -1.025558 \\
\hline $\mathrm{H}$ & -0.705309 & -2.886381 & -1.678173 \\
\hline $\mathrm{H}$ & -0.697806 & -3.609482 & 2.572263 \\
\hline $\mathrm{H}$ & -1.911236 & -5.715701 & 2.202940 \\
\hline $\mathrm{H}$ & -1.907931 & -4.999029 & -2.036519 \\
\hline $\mathrm{H}$ & -2.526969 & -6.428867 & -0.095465 \\
\hline $\mathrm{C}$ & 1.444354 & 3.378599 & -0.715003 \\
\hline $\mathrm{C}$ & 3.102931 & 5.080717 & -2.186213 \\
\hline C & 1.944967 & 4.552946 & -0.135504 \\
\hline $\mathrm{C}$ & 1.795999 & 3.060164 & -2.028230 \\
\hline C & 2.624506 & 3.906730 & -2.762267 \\
\hline $\mathrm{C}$ & 2.763344 & 5.402551 & -0.872444 \\
\hline $\mathrm{H}$ & 1.408082 & 2.148033 & -2.478951 \\
\hline $\mathrm{H}$ & 2.879519 & 3.658638 & -3.791339 \\
\hline $\mathrm{H}$ & 3.135663 & 6.320073 & -0.421383 \\
\hline $\mathrm{H}$ & 3.737509 & 5.750751 & -2.762565 \\
\hline $\mathrm{C}$ & -0.925079 & 3.289944 & 0.981254 \\
\hline $\mathrm{C}$ & -3.165349 & 4.693444 & 1.885894 \\
\hline C & -1.216118 & 4.557061 & 0.468575 \\
\hline C & -1.784428 & 2.717342 & 1.930486 \\
\hline C & -2.888975 & 3.423094 & 2.389080 \\
\hline C & -2.333035 & 5.254282 & 0.923502 \\
\hline $\mathrm{H}$ & -0.587010 & 5.001339 & -0.301288 \\
\hline $\mathrm{H}$ & -1.587718 & 1.714203 & 2.314664 \\
\hline $\mathrm{H}$ & -3.541542 & 2.978182 & 3.138308 \\
\hline $\mathrm{H}$ & -2.551098 & 6.240468 & 0.519027 \\
\hline $\mathrm{H}$ & -4.034616 & 5.242627 & 2.241142 \\
\hline $\mathrm{C}$ & 1.519752 & 1.838729 & 1.704777 \\
\hline C & 3.060823 & 1.108073 & 3.946236 \\
\hline C & 1.244149 & 2.355284 & 2.975635 \\
\hline C & 2.615814 & 0.963990 & 1.564977 \\
\hline $\mathrm{C}$ & 3.351386 & 0.590608 & 2.691045 \\
\hline $\mathrm{C}$ & 2.010404 & 2.006912 & 4.082648 \\
\hline $\mathrm{H}$ & 0.411342 & 3.041911 & 3.110578 \\
\hline $\mathrm{H}$ & 4.166111 & -0.130213 & 2.585522 \\
\hline $\mathrm{H}$ & 1.771748 & 2.430259 & 5.055779 \\
\hline $\mathrm{H}$ & 3.647038 & 0.806193 & 4.811405 \\
\hline $\mathrm{C}$ & 0.179066 & -1.289488 & 2.533017 \\
\hline C & -0.190635 & -1.102938 & 5.295634 \\
\hline $\mathrm{C}$ & -1.004176 & -0.754309 & 3.054908 \\
\hline C & 1.169021 & -1.744469 & 3.405729 \\
\hline C & 0.982024 & -1.649503 & 4.782684 \\
\hline C & -1.186929 & -0.657438 & 4.429012 \\
\hline $\mathrm{H}$ & -1.798192 & -0.457478 & 2.369013 \\
\hline $\mathrm{H}$ & 2.084251 & -2.190104 & 3.013118 \\
\hline $\mathrm{H}$ & 1.753733 & -2.014412 & 5.458411 \\
\hline $\mathrm{H}$ & -2.115160 & -0.245635 & 4.824516 \\
\hline $\mathrm{H}$ & -0.334482 & -1.037145 & 6.372235 \\
\hline $\mathrm{H}$ & 1.692076 & 4.807349 & 0.894819 \\
\hline $\mathrm{H}$ & 1.884367 & -4.167467 & 0.416991 \\
\hline C & 2.503331 & -3.279172 & 0.333174 \\
\hline C & 2.015625 & -1.934796 & 0.398505 \\
\hline $\mathrm{H}$ & 4.535721 & -4.111545 & -0.083020 \\
\hline C & 3.142107 & -1.057760 & 0.159364 \\
\hline C & 4.297577 & -1.891344 & -0.023860 \\
\hline $\mathrm{H}$ & 5.314282 & -1.582895 & -0.258077 \\
\hline C & 3.894249 & -3.250562 & 0.071018 \\
\hline $\mathrm{Fe}$ & 2.862551 & -2.282907 & -1.415092 \\
\hline $\mathrm{H}$ & 0.476786 & -1.514460 & -2.711148 \\
\hline C & 1.461746 & -1.947403 & -2.872581 \\
\hline C & 1.812792 & -3.327880 & -2.821645 \\
\hline $\mathrm{H}$ & 2.690758 & -0.122872 & -3.250237 \\
\hline $\mathrm{H}$ & 1.147358 & -4.152000 & -2.584996 \\
\hline C & 3.209879 & -3.433502 & -3.069000 \\
\hline $\mathrm{H}$ & 3.790074 & -4.349911 & -3.060831 \\
\hline
\end{tabular}

\begin{tabular}{|c|c|c|c|}
\hline C & 3.724224 & -2.122058 & -3.268607 \\
\hline $\mathrm{H}$ & 4.763419 & -1.876589 & -3.466745 \\
\hline C & 2.640432 & -1.203880 & -3.147417 \\
\hline C & 3.084643 & 0.448541 & 0.226341 \\
\hline $\mathrm{H}$ & 2.456986 & 0.841672 & -0.584816 \\
\hline $\mathrm{N}$ & 4.490675 & 1.026283 & -0.076716 \\
\hline C & 4.716714 & 2.441816 & 0.361468 \\
\hline $\mathrm{H}$ & 4.577759 & 2.527549 & 1.440005 \\
\hline $\mathrm{H}$ & 4.007073 & 3.087091 & -0.162202 \\
\hline $\mathrm{H}$ & 5.740592 & 2.712734 & 0.090041 \\
\hline $\mathrm{C}$ & 4.847886 & 0.906824 & -1.525344 \\
\hline $\mathrm{H}$ & 4.620786 & -0.103829 & -1.870141 \\
\hline $\mathrm{H}$ & 5.910845 & 1.133351 & -1.646140 \\
\hline $\mathrm{H}$ & 4.247881 & 1.643055 & -2.071201 \\
\hline $\mathrm{Pd}$ & -0.645398 & 0.387838 & -0.644436 \\
\hline C & -1.565329 & 1.720484 & -1.919104 \\
\hline $\mathrm{C}$ & -1.109478 & 1.815851 & -3.165537 \\
\hline $\mathrm{H}$ & -0.167803 & 1.372078 & -3.496701 \\
\hline $\mathrm{C}$ & -2.884300 & 2.306082 & -1.525115 \\
\hline $\mathrm{H}$ & -3.658482 & 1.520945 & -1.554077 \\
\hline $\mathrm{H}$ & -2.880925 & 2.721956 & -0.508542 \\
\hline C & -3.243487 & 3.398405 & -2.532803 \\
\hline $\mathrm{H}$ & -2.549618 & 4.254318 & -2.417118 \\
\hline $\mathrm{H}$ & -4.265991 & 3.758617 & -2.374748 \\
\hline $\mathrm{O}$ & -3.186248 & 2.886060 & -3.836789 \\
\hline $\mathrm{C}$ & -1.886166 & 2.560406 & -4.228910 \\
\hline $\mathrm{H}$ & -1.971561 & 1.952739 & -5.138725 \\
\hline $\mathrm{H}$ & -1.317157 & 3.474700 & -4.497446 \\
\hline $\mathrm{O}$ & -2.962369 & -1.130078 & 0.498956 \\
\hline B & -2.904464 & -0.974035 & -0.844451 \\
\hline $\mathrm{O}$ & -4.075262 & -1.010374 & -1.499593 \\
\hline $\mathrm{C}$ & -5.055244 & -1.502106 & -0.525412 \\
\hline C & -4.383675 & -1.148621 & 0.843497 \\
\hline C & -4.582895 & -2.176574 & 1.935356 \\
\hline $\mathrm{H}$ & -4.184599 & -3.155317 & 1.646269 \\
\hline $\mathrm{H}$ & -5.648726 & -2.285752 & 2.176300 \\
\hline $\mathrm{H}$ & -4.062105 & -1.858253 & 2.848012 \\
\hline C & -4.710702 & 0.245062 & 1.351936 \\
\hline $\mathrm{H}$ & -4.522466 & 1.014773 & 0.591070 \\
\hline $\mathrm{H}$ & -4.072446 & 0.465958 & 2.219539 \\
\hline $\mathrm{H}$ & -5.755763 & 0.322088 & 1.675282 \\
\hline C & -5.169818 & -2.995653 & -0.770736 \\
\hline $\mathrm{H}$ & -4.213184 & -3.508355 & -0.595768 \\
\hline $\mathrm{H}$ & -5.459502 & -3.163041 & -1.814451 \\
\hline $\mathrm{H}$ & -5.932034 & -3.451132 & -0.127053 \\
\hline C & -6.373298 & -0.809818 & -0.784016 \\
\hline $\mathrm{H}$ & -6.274823 & 0.281258 & -0.773471 \\
\hline $\mathrm{H}$ & -7.114599 & -1.103119 & -0.028349 \\
\hline $\mathrm{H}$ & -6.759698 & -1.104283 & -1.766342 \\
\hline $\mathrm{H}$ & -1.852409 & -0.863257 & -1.470881 \\
\hline $\mathrm{H}$ & 5.143538 & 0.440147 & 0.457683 \\
\hline
\end{tabular}

SCF energy: -3329.871685250

M06-D3 (Gas Phase) /6-31G(d)+SDD (Pd, Fe) opt. No imaginary frequency 


\begin{tabular}{|c|c|c|c|}
\hline \multicolumn{4}{|c|}{ Int-5STania_conf 6} \\
\hline $\mathrm{P}$ & 0.290826 & -1.564881 & 0.752661 \\
\hline $\mathrm{P}$ & 0.315510 & 2.194284 & 0.327154 \\
\hline $\mathrm{C}$ & -0.567880 & -3.181169 & 0.541017 \\
\hline C & -1.947323 & -5.600395 & 0.203772 \\
\hline C & -0.930057 & -3.628130 & -0.735150 \\
\hline C & -0.910993 & -3.964138 & 1.647631 \\
\hline $\mathrm{C}$ & -1.597425 & -5.164417 & 1.476677 \\
\hline $\mathrm{C}$ & -1.607252 & -4.829480 & -0.906181 \\
\hline $\mathrm{H}$ & -0.689805 & -3.033305 & -1.613593 \\
\hline $\mathrm{H}$ & -0.651491 & -3.642853 & 2.654879 \\
\hline $\mathrm{H}$ & -1.858057 & -5.760236 & 2.349096 \\
\hline $\mathrm{H}$ & -1.881342 & -5.157335 & -1.907655 \\
\hline $\mathrm{H}$ & -2.485011 & -6.537382 & 0.074475 \\
\hline $\mathrm{C}$ & 1.264611 & 3.317228 & -0.754058 \\
\hline C & 2.752191 & 5.019390 & -2.395012 \\
\hline C & 1.820112 & 4.493361 & -0.231406 \\
\hline C & 1.467904 & 3.000604 & -2.098857 \\
\hline C & 2. 213259 & 3.846679 & -2.917731 \\
\hline $\mathrm{C}$ & 2.553197 & 5.343393 & -1.052834 \\
\hline $\mathrm{H}$ & 1.031908 & 2.087083 & -2.502861 \\
\hline $\mathrm{H}$ & 2.355573 & 3.599487 & -3.968411 \\
\hline $\mathrm{H}$ & 2.969197 & 6.263008 & -0.646214 \\
\hline $\mathrm{H}$ & 3. 322295 & 5.689053 & -3.035628 \\
\hline $\mathrm{C}$ & -0.991335 & 3.206596 & 1.086851 \\
\hline $\mathrm{C}$ & -3.203487 & 4.568919 & 2.111941 \\
\hline C & -1.302860 & 4.488885 & 0.625923 \\
\hline C & -1.810372 & 2.603456 & 2.051060 \\
\hline C & -2.903184 & 3.286677 & 2.568440 \\
\hline C & -2.404890 & 5.165447 & 1.141594 \\
\hline $\mathrm{H}$ & -0.705756 & 4.957870 & -0.154243 \\
\hline $\mathrm{H}$ & -1.585970 & 1.594926 & 2.401831 \\
\hline $\mathrm{H}$ & -3.525118 & 2.815969 & 3.327987 \\
\hline $\mathrm{H}$ & -2.641032 & 6.162890 & 0.777094 \\
\hline $\mathrm{H}$ & -4.063682 & 5.101596 & 2.511727 \\
\hline $\mathrm{C}$ & 1.507178 & 1.812726 & 1.697699 \\
\hline $\mathrm{C}$ & 3.162741 & 1.133437 & 3.873938 \\
\hline $\mathrm{C}$ & 1.269301 & 2.322135 & 2.979570 \\
\hline $\mathrm{C}$ & 2.617806 & 0.964492 & 1.514832 \\
\hline $\mathrm{C}$ & 3.412477 & 0.617952 & 2.609187 \\
\hline $\mathrm{C}$ & 2.091453 & 1.999414 & 4.053769 \\
\hline $\mathrm{H}$ & 0.423797 & 2.985046 & 3.148707 \\
\hline $\mathrm{H}$ & 4.241125 & -0.081078 & 2.471273 \\
\hline $\mathrm{H}$ & 1.880653 & 2.417218 & 5.035691 \\
\hline $\mathrm{H}$ & 3.795731 & 0.853749 & 4.713247 \\
\hline $\mathrm{C}$ & 0.186527 & -1.324261 & 2.558797 \\
\hline $\mathrm{C}$ & -0.184134 & -1.062577 & 5.312477 \\
\hline $\mathrm{C}$ & -1.027712 & -0.843475 & 3.065337 \\
\hline $\mathrm{C}$ & 1.203595 & -1.692031 & 3.439970 \\
\hline $\mathrm{C}$ & 1.016523 & -1.558347 & 4.813905 \\
\hline $\mathrm{C}$ & -1.209118 & -0.709188 & 4.436297 \\
\hline $\mathrm{H}$ & -1.842196 & -0.615227 & 2.374249 \\
\hline $\mathrm{H}$ & 2.139609 & -2.101078 & 3.056737 \\
\hline $\mathrm{H}$ & 1.810343 & -1.853307 & 5.498169 \\
\hline $\mathrm{H}$ & -2.158414 & -0.341334 & 4.824187 \\
\hline $\mathrm{H}$ & -0.328103 & -0.965681 & 6.386705 \\
\hline $\mathrm{H}$ & 1.676817 & 4.747370 & 0.820196 \\
\hline $\mathrm{H}$ & 1.967941 & -4.202173 & 0.493748 \\
\hline $\mathrm{C}$ & 2.558702 & -3.299412 & 0.370055 \\
\hline $\mathrm{C}$ & 2.034381 & -1.968742 & 0.414514 \\
\hline $\mathrm{H}$ & 4.603063 & -4.083660 & -0.080334 \\
\hline $\mathrm{C}$ & 3.127107 & -1.065256 & 0.119245 \\
\hline $\mathrm{C}$ & 4.301136 & -1.870259 & -0.072487 \\
\hline $\mathrm{H}$ & 5.301694 & -1.539948 & -0.344275 \\
\hline $\mathrm{C}$ & 3.940728 & -3.237729 & 0.068824 \\
\hline $\mathrm{Fe}$ & 2.844998 & -2.340848 & -1.414959 \\
\hline $\mathrm{H}$ & 0.408460 & -1.697871 & -2.690483 \\
\hline $\mathrm{C}$ & 1.405209 & -2.099778 & -2.855175 \\
\hline $\mathrm{C}$ & 1.807759 & -3.464222 & -2.770069 \\
\hline $\mathrm{H}$ & 2.553316 & -0.243090 & -3.311113 \\
\hline $\mathrm{H}$ & 1.177663 & -4.304684 & -2.497103 \\
\hline $\mathrm{C}$ & 3.202841 & -3.525341 & -3.042104 \\
\hline $\mathrm{H}$ & 3.817319 & -4.418886 & -3.019037 \\
\hline
\end{tabular}

\begin{tabular}{|c|c|c|c|}
\hline $\mathrm{C}$ & 3.663739 & -2.202165 & -3.289859 \\
\hline $\mathrm{H}$ & 4.689329 & -1.925044 & -3.515376 \\
\hline $\mathrm{C}$ & 2.548735 & -1.321456 & -3.174533 \\
\hline C & 3.032437 & 0.440543 & 0.161464 \\
\hline $\mathrm{H}$ & 2.352516 & 0.802047 & -0.622758 \\
\hline $\mathrm{N}$ & 4.406121 & 1.048298 & -0.221790 \\
\hline $\mathrm{C}$ & 4.620537 & 2.474229 & 0.184747 \\
\hline $\mathrm{H}$ & 4.513038 & 2.577587 & 1.265366 \\
\hline $\mathrm{H}$ & 3.884089 & 3.097218 & -0.329004 \\
\hline $\mathrm{H}$ & 5.630627 & 2.757968 & -0.122875 \\
\hline $\mathrm{C}$ & 4.688863 & 0.918125 & -1.685173 \\
\hline $\mathrm{H}$ & 4.492969 & -0.108966 & -1.998440 \\
\hline $\mathrm{H}$ & 5.731200 & 1.192713 & -1.869164 \\
\hline $\mathrm{H}$ & 4.023927 & 1.613759 & -2.209426 \\
\hline $\mathrm{Pd}$ & -0.654171 & 0.289689 & -0.602126 \\
\hline C & -1.651280 & 1.547130 & -1.882473 \\
\hline $\mathrm{C}$ & -2.649502 & 2.337829 & -1.500413 \\
\hline $\mathrm{H}$ & -2.855196 & 2.568031 & -0.454155 \\
\hline $\mathrm{C}$ & -1.422893 & 1.224845 & -3.330802 \\
\hline $\mathrm{H}$ & -0.357459 & 1.139910 & -3.596819 \\
\hline $\mathrm{H}$ & -1.880840 & 0.252287 & -3.576712 \\
\hline C & -2.080920 & 2.322942 & -4.171418 \\
\hline $\mathrm{H}$ & -2.072072 & 2.061397 & -5.235183 \\
\hline $\mathrm{H}$ & -1.528832 & 3.274391 & -4.039836 \\
\hline O & -3.422786 & 2.470877 & -3.797845 \\
\hline $\mathrm{C}$ & -3.569137 & 2.982963 & -2.505029 \\
\hline $\mathrm{H}$ & -3.396034 & 4.079408 & -2.491172 \\
\hline $\mathrm{H}$ & -4.617284 & 2.820705 & -2.217193 \\
\hline $\mathrm{O}$ & -3.015072 & -1.131319 & 0.388085 \\
\hline B & -2.829964 & -1.054876 & -0.951387 \\
\hline $\mathrm{O}$ & -3.927890 & -1.135231 & -1.717718 \\
\hline C & -5.000726 & -1.555650 & -0.809756 \\
\hline $\mathrm{C}$ & -4.465123 & -1.091779 & 0.584293 \\
\hline C & -4.800431 & -2.009739 & 1.738365 \\
\hline $\mathrm{H}$ & -4.378997 & -3.010775 & 1.593900 \\
\hline $\mathrm{H}$ & -5.887922 & -2.098547 & 1.862451 \\
\hline $\mathrm{H}$ & -4.389637 & -1.601786 & 2.671421 \\
\hline C & -4.805937 & 0.348864 & 0.921026 \\
\hline $\mathrm{H}$ & -4.552173 & 1.029152 & 0.096386 \\
\hline $\mathrm{H}$ & -4.224931 & 0.655025 & 1.802174 \\
\hline $\mathrm{H}$ & -5.870560 & 0.467095 & 1.156122 \\
\hline $\mathrm{C}$ & -5.094298 & -3.065143 & -0.942156 \\
\hline $\mathrm{H}$ & -4.163965 & -3.558282 & -0.625508 \\
\hline $\mathrm{H}$ & -5.274405 & -3.319700 & -1.992992 \\
\hline $\mathrm{H}$ & -5.922058 & -3.468936 & -0.346559 \\
\hline $\mathrm{C}$ & -6.285111 & -0.892805 & -1.250004 \\
\hline $\mathrm{H}$ & -6.178941 & 0.194515 & -1.328570 \\
\hline $\mathrm{H}$ & -7.095473 & -1.118454 & -0.543620 \\
\hline $\mathrm{H}$ & -6.580494 & -1.273996 & -2.234117 \\
\hline $\mathrm{H}$ & -1.715695 & -1.035128 & -1.480989 \\
\hline $\mathrm{H}$ & 5.100491 & 0.485672 & 0.284816 \\
\hline
\end{tabular}

SCF energy: -3329.875180110

M06-D3 (Gas Phase) /6-31G(d)+SDD (Pd, Fe) opt. No imaginary frequency 


\begin{tabular}{|c|c|c|c|}
\hline \multicolumn{4}{|c|}{ Int-5STania_conf 4} \\
\hline P & 0.080867 & 2.157839 & 0.513140 \\
\hline $\mathrm{C}$ & -0.397641 & -3.302007 & 0.322439 \\
\hline C & -1.551636 & -5.797692 & -0.236175 \\
\hline $\mathrm{C}$ & -0.765880 & -3.645734 & -0.983579 \\
\hline $\mathrm{C}$ & -0.621276 & -4.229450 & 1.346795 \\
\hline C & -1.191724 & -5.468167 & 1.065982 \\
\hline C & -1.335305 & -4.882814 & -1.263802 \\
\hline $\mathrm{H}$ & -0.608768 & -2.945562 & -1.801317 \\
\hline $\mathrm{H}$ & -0.357451 & -3.989539 & 2.375708 \\
\hline $\mathrm{H}$ & -1.358017 & -6.176722 & 1.874856 \\
\hline $\mathrm{H}$ & -1.615304 & -5.128905 & -2.286542 \\
\hline $\mathrm{H}$ & -2.001907 & -6.764492 & -0.451292 \\
\hline $\mathrm{C}$ & 1.126893 & 3.437392 & -0.294492 \\
\hline C & 2.796227 & 5.351416 & -1.485399 \\
\hline C & 2.070146 & 4.143686 & 0.476069 \\
\hline C & 1.005431 & 3.737030 & -1.656050 \\
\hline C & 1.837076 & 4.687536 & -2.244430 \\
\hline C & 2.904715 & 5.087181 & -0.120510 \\
\hline $\mathrm{H}$ & 0.239195 & 3.250212 & -2.254963 \\
\hline $\mathrm{H}$ & 1.725966 & 4.912119 & -3.303254 \\
\hline $\mathrm{H}$ & 3.623799 & 5.632662 & 0.488269 \\
\hline $\mathrm{H}$ & 3.444475 & 6.091681 & -1.949785 \\
\hline C & -1.342410 & 3.027241 & 1.223752 \\
\hline C & -3.608584 & 4.288795 & 2.238291 \\
\hline C & -1.428697 & 4.421680 & 1.213890 \\
\hline C & -2.399998 & 2.263290 & 1.737136 \\
\hline C & -3.523678 & 2.897211 & 2.251419 \\
\hline C & -2.564299 & 5.047831 & 1.719806 \\
\hline $\mathrm{H}$ & -0.620016 & 5.024356 & 0.802273 \\
\hline $\mathrm{H}$ & -2.351494 & 1.172464 & 1.714865 \\
\hline $\mathrm{H}$ & -4.340197 & 2.304889 & 2.661309 \\
\hline $\mathrm{H}$ & -2.632338 & 6.133423 & 1.706150 \\
\hline $\mathrm{H}$ & -4.494730 & 4.782322 & 2.631720 \\
\hline $\mathrm{C}$ & 1.141212 & 1.663628 & 1.930985 \\
\hline $\mathrm{C}$ & 2.703140 & 0.877295 & 4.118474 \\
\hline $\mathrm{C}$ & 0.749104 & 1.972551 & 3.235420 \\
\hline $\mathrm{C}$ & 2.335529 & 0.944210 & 1.722778 \\
\hline $\mathrm{C}$ & 3.090408 & 0.543512 & 2.825712 \\
\hline $\mathrm{C}$ & 1.529864 & 1.592183 & 4.322577 \\
\hline $\mathrm{H}$ & -0.177228 & 2.517701 & 3.408494 \\
\hline $\mathrm{H}$ & 3.980742 & -0.068444 & 2.672173 \\
\hline $\mathrm{H}$ & 1.207806 & 1.842714 & 5.330960 \\
\hline $\mathrm{H}$ & 3.307342 & 0.558453 & 4.965156 \\
\hline $\mathrm{C}$ & 0.112514 & -1.554974 & 2.501272 \\
\hline $\mathrm{C}$ & -0.447230 & -1.566863 & 5.235948 \\
\hline $\mathrm{C}$ & -1.148046 & -1.160756 & 2.966131 \\
\hline $\mathrm{C}$ & 1.083934 & -1.969389 & 3.413868 \\
\hline $\mathrm{C}$ & 0.801740 & -1.974862 & 4.777815 \\
\hline $\mathrm{C}$ & -1.423727 & -1.161925 & 4. 327911 \\
\hline $\mathrm{H}$ & -1.925211 & -0.896825 & 2.246560 \\
\hline $\mathrm{H}$ & 2.059777 & -2.306298 & 3.060834 \\
\hline $\mathrm{H}$ & 1.559811 & -2.308419 & 5.484546 \\
\hline $\mathrm{H}$ & -2.407714 & -0.858770 & 4. 682512 \\
\hline $\mathrm{H}$ & -0.665428 & -1.578152 & 6.302011 \\
\hline $\mathrm{H}$ & 2.148474 & 3.961136 & 1.549454 \\
\hline $\mathrm{H}$ & 2.290982 & -4.139848 & 0.503341 \\
\hline $\mathrm{C}$ & 2.787509 & -3.177310 & 0.418813 \\
\hline $\mathrm{C}$ & 2.126110 & -1.908610 & 0.437980 \\
\hline $\mathrm{H}$ & 4.923167 & -3.735827 & 0.063684 \\
\hline $\mathrm{C}$ & 3.138927 & -0.892879 & 0.214155 \\
\hline $\mathrm{C}$ & 4.394097 & -1.564095 & 0.071368 \\
\hline $\mathrm{H}$ & 5.359453 & -1.118942 & -0.150390 \\
\hline C & 4.170179 & -2.964683 & 0.187370 \\
\hline $\mathrm{Fe}$ & 3.059800 & -2.191322 & -1.342326 \\
\hline $\mathrm{H}$ & 0.675755 & -1.747787 & -2.789761 \\
\hline $\mathrm{C}$ & 1.702565 & -2.090436 & -2.876996 \\
\hline $\mathrm{C}$ & 2.178148 & -3.422770 & -2.708270 \\
\hline $\mathrm{H}$ & 2.764871 & -0.192906 & -3.364265 \\
\hline $\mathrm{H}$ & 1.580228 & -4.287008 & -2.435582 \\
\hline C & 3.589369 & -3.406442 & -2.894114 \\
\hline $\mathrm{H}$ & 4.253738 & -4.257838 & -2.791447 \\
\hline $\mathrm{C}$ & 3.986002 & -2.067356 & -3.168291 \\
\hline
\end{tabular}

\begin{tabular}{|c|c|c|c|}
\hline $\mathrm{H}$ & 5.004336 & -1.730027 & -3.335864 \\
\hline $\mathrm{C}$ & 2.815326 & -1.257022 & -3.161904 \\
\hline $\mathrm{C}$ & 2.780432 & 0.558696 & 0.327570 \\
\hline $\mathrm{H}$ & 1.946044 & 0.727133 & -0.372659 \\
\hline $\mathrm{N}$ & 3.822376 & 1.574914 & -0.174442 \\
\hline $\mathrm{C}$ & 4.098740 & 1.461786 & -1.634215 \\
\hline $\mathrm{H}$ & 3.147769 & 1.563406 & -2.166841 \\
\hline $\mathrm{H}$ & 4.548764 & 0.488619 & -1.845263 \\
\hline $\mathrm{H}$ & 4.770182 & 2.275347 & -1.923603 \\
\hline $\mathrm{C}$ & 5.079260 & 1.718653 & 0.617006 \\
\hline $\mathrm{H}$ & 4.825006 & 1.919155 & 1.659855 \\
\hline $\mathrm{H}$ & 5.637197 & 2.565060 & 0.205650 \\
\hline $\mathrm{H}$ & 5.677161 & 0.808749 & 0.540467 \\
\hline $\mathrm{Pd}$ & -0.632149 & 0.259812 & -0.634565 \\
\hline $\mathrm{H}$ & 3.325738 & 2.475901 & -0.058539 \\
\hline $\mathrm{P}$ & 0.336557 & -1.652878 & 0.690123 \\
\hline $\mathrm{C}$ & -1.442703 & 1.543182 & -2.003327 \\
\hline C & -2.362432 & 2.488363 & -1.829676 \\
\hline $\mathrm{H}$ & -2.766858 & 2.753035 & -0.850990 \\
\hline $\mathrm{C}$ & -0.942983 & 1.142777 & -3.362392 \\
\hline $\mathrm{H}$ & 0.100630 & 1.468533 & -3.518026 \\
\hline $\mathrm{H}$ & -0.944305 & 0.048306 & -3.489483 \\
\hline C & -1.864692 & 1.758921 & -4.422928 \\
\hline $\mathrm{H}$ & -2.823522 & 1.208066 & -4.434037 \\
\hline $\mathrm{H}$ & -1.415865 & 1.695721 & -5.420041 \\
\hline $\mathrm{O}$ & -2.080558 & 3.116519 & -4.143714 \\
\hline $\mathrm{C}$ & -2.880811 & 3.279442 & -3.005144 \\
\hline $\mathrm{H}$ & -2.879049 & 4.351154 & -2.766228 \\
\hline $\mathrm{H}$ & -3.929868 & 2.993607 & -3.217769 \\
\hline O & -3.087126 & -1.057742 & 0.225538 \\
\hline B & -2.796655 & -1.038507 & -1.098264 \\
\hline $\mathrm{O}$ & -3.837633 & -1.103583 & -1.943446 \\
\hline $\mathrm{C}$ & -5.030144 & -0.973542 & -1.097999 \\
\hline C & -4.500867 & -1.439693 & 0.294953 \\
\hline C & -4.507434 & -2.946198 & 0.487187 \\
\hline $\mathrm{H}$ & -4.060588 & -3.467050 & -0.370531 \\
\hline $\mathrm{H}$ & -5.523708 & -3.329952 & 0.637599 \\
\hline $\mathrm{H}$ & -3.909893 & -3.192206 & 1.375466 \\
\hline C & -5.124363 & -0.742818 & 1.482230 \\
\hline $\mathrm{H}$ & -4.975066 & 0.342452 & 1.439099 \\
\hline $\mathrm{H}$ & -4.678943 & -1.121256 & 2.412063 \\
\hline $\mathrm{H}$ & -6.203219 & -0.942264 & 1.526517 \\
\hline $\mathrm{C}$ & -6.129650 & -1.832036 & -1.677026 \\
\hline $\mathrm{H}$ & -5.809690 & -2.869931 & -1.813782 \\
\hline $\mathrm{H}$ & -6.434643 & -1.439227 & -2.653875 \\
\hline $\mathrm{H}$ & -7.009997 & -1.820521 & -1.020236 \\
\hline $\mathrm{C}$ & -5.411210 & 0.496254 & -1.135627 \\
\hline $\mathrm{H}$ & -4.627667 & 1.130824 & -0.696216 \\
\hline $\mathrm{H}$ & -6.351298 & 0.680825 & -0.601439 \\
\hline $\mathrm{H}$ & -5.548004 & 0.799307 & -2.181087 \\
\hline $\mathrm{H}$ & -1.651201 & -1.085062 & -1.543598 \\
\hline
\end{tabular}

SCF energy: -3329.877656300

M06-D3 (Gas Phase)/6-31G(d)+SDD (Pd, Fe) opt. No imaginary frequency 


\begin{tabular}{|c|c|c|c|}
\hline \multicolumn{4}{|c|}{ Int-5RTania_conf1 } \\
\hline P & 0.284326 & -1.589414 & 0.689955 \\
\hline P & 0.239329 & 2.180285 & 0.466088 \\
\hline C & -0.488231 & -3.225595 & 0.344738 \\
\hline $\mathrm{C}$ & -1.674730 & -5.711262 & -0.183514 \\
\hline C & -0.907765 & -3.558476 & -0.947753 \\
\hline C & -0.676920 & -4.156755 & 1.371906 \\
\hline $\mathrm{C}$ & -1.265146 & -5.390844 & 1.106389 \\
\hline $\mathrm{C}$ & -1.492680 & -4.791338 & -1.213188 \\
\hline $\mathrm{H}$ & -0.786880 & -2.849793 & -1.764810 \\
\hline $\mathrm{H}$ & -0.368094 & -3.925318 & 2.390307 \\
\hline $\mathrm{H}$ & -1.403840 & -6.103623 & 1.916816 \\
\hline $\mathrm{H}$ & -1.814269 & -5.030034 & -2.225517 \\
\hline $\mathrm{H}$ & -2.137365 & -6.674726 & -0.387029 \\
\hline C & 1.182540 & 3.493975 & -0.383075 \\
\hline C & 2.673403 & 5.502064 & -1.626452 \\
\hline C & 1.683253 & 4.569679 & 0.365903 \\
\hline C & 1.441805 & 3.430071 & -1.755065 \\
\hline C & 2.189758 & 4.431234 & -2.372627 \\
\hline C & 2.416876 & 5.572850 & -0.257011 \\
\hline $\mathrm{H}$ & 1.027313 & 2.610647 & -2.343010 \\
\hline $\mathrm{H}$ & 2.374331 & 4.384523 & -3.444248 \\
\hline $\mathrm{H}$ & 2.789095 & 6.412851 & 0.326042 \\
\hline $\mathrm{H}$ & 3.244915 & 6.290192 & -2.112421 \\
\hline C & -1.201711 & 2.983768 & 1.238687 \\
\hline C & -3.544391 & 4.058425 & 2.313809 \\
\hline C & -1.503187 & 4.335704 & 1.055510 \\
\hline C & -2.088015 & 2.171158 & 1.958761 \\
\hline C & -3.244802 & 2.710828 & 2.505337 \\
\hline C & -2.674719 & 4.866076 & 1.588980 \\
\hline $\mathrm{H}$ & -0.840067 & 4.979698 & 0.480060 \\
\hline $\mathrm{H}$ & -1.867280 & 1.111813 & 2.095784 \\
\hline $\mathrm{H}$ & -3.918366 & 2.076453 & 3.080668 \\
\hline $\mathrm{H}$ & -2.905806 & 5.917945 & 1.435523 \\
\hline $\mathrm{H}$ & -4.456614 & 4.478747 & 2.731794 \\
\hline $\mathrm{C}$ & 1.361675 & 1.718396 & 1.861499 \\
\hline $\mathrm{C}$ & 2.979366 & 0.982068 & 4.039342 \\
\hline C & 1.025862 & 2.088195 & 3.168612 \\
\hline $\mathrm{C}$ & 2.534129 & 0.966067 & 1.654805 \\
\hline $\mathrm{C}$ & 3.314842 & 0.591518 & 2.749704 \\
\hline $\mathrm{C}$ & 1.830831 & 1.736616 & 4.246490 \\
\hline $\mathrm{H}$ & 0.118402 & 2.660728 & 3.351419 \\
\hline $\mathrm{H}$ & 4.197308 & -0.034118 & 2.593882 \\
\hline $\mathrm{H}$ & 1.548100 & 2.042104 & 5.251442 \\
\hline $\mathrm{H}$ & 3.603656 & 0.684690 & 4.879151 \\
\hline $\mathrm{C}$ & 0.153308 & -1.500692 & 2.511670 \\
\hline $\mathrm{C}$ & -0.235973 & -1.481069 & 5.277815 \\
\hline $\mathrm{C}$ & -1.096634 & -1.168749 & 3.048828 \\
\hline $\mathrm{C}$ & 1.198584 & -1.845173 & 3.369806 \\
\hline $\mathrm{C}$ & 1.001363 & -1.834129 & 4.748803 \\
\hline $\mathrm{C}$ & -1.287660 & -1.151821 & 4.424559 \\
\hline $\mathrm{H}$ & -1.930043 & -0.968483 & 2.374333 \\
\hline $\mathrm{H}$ & 2.166815 & -2.141973 & 2.965131 \\
\hline $\mathrm{H}$ & 1.818222 & -2.113406 & 5.412403 \\
\hline $\mathrm{H}$ & -2.264900 & -0.895768 & 4.832147 \\
\hline $\mathrm{H}$ & -0.386391 & -1.478535 & 6.355528 \\
\hline $\mathrm{H}$ & 1.500733 & 4.622697 & 1.440531 \\
\hline $\mathrm{H}$ & 2.063144 & -4.142887 & 0.336738 \\
\hline $\mathrm{C}$ & 2.624993 & -3.215756 & 0.275232 \\
\hline C & 2.054184 & -1.906274 & 0.372628 \\
\hline $\mathrm{H}$ & 4.702005 & -3.911452 & -0.170781 \\
\hline $\mathrm{C}$ & 3.124771 & -0.955002 & 0.150323 \\
\hline $\mathrm{C}$ & 4.327440 & -1.712230 & -0.057917 \\
\hline $\mathrm{H}$ & 5.321328 & -1.336879 & -0.293619 \\
\hline $\mathrm{C}$ & 4.009947 & -3.095490 & 0.007544 \\
\hline $\mathrm{Fe}$ & 2.910704 & -2.162424 & -1.450542 \\
\hline $\mathrm{H}$ & 0.465161 & -1.559438 & -2.710085 \\
\hline C & 1.477866 & -1.910824 & -2.890445 \\
\hline $\mathrm{C}$ & 1.927829 & -3.262185 & -2.862022 \\
\hline $\mathrm{H}$ & 2.561553 & 0.006839 & -3.251030 \\
\hline $\mathrm{H}$ & 1.323083 & -4.132889 & -2.628278 \\
\hline C & 3.326480 & -3.260024 & -3.123778 \\
\hline $\mathrm{H}$ & 3.973496 & -4.130565 & -3.134307 \\
\hline
\end{tabular}

\begin{tabular}{|c|c|c|c|}
\hline $\mathrm{C}$ & 3.740876 & -1.911094 & -3.309020 \\
\hline $\mathrm{H}$ & 4.757207 & -1.587183 & -3.512901 \\
\hline $\mathrm{C}$ & 2.594314 & -1.076428 & -3.164252 \\
\hline C & 2.993167 & 0.544115 & 0.279129 \\
\hline $\mathrm{H}$ & 2.314797 & 0.935707 & -0.492717 \\
\hline $\mathrm{N}$ & 4.352610 & 1.205569 & -0.049140 \\
\hline $\mathrm{C}$ & 4.526590 & 2.608958 & 0.445059 \\
\hline $\mathrm{H}$ & 4.397930 & 2.644114 & 1.527786 \\
\hline $\mathrm{H}$ & 3.783368 & 3.243588 & -0.043387 \\
\hline $\mathrm{H}$ & 5.534480 & 2.933745 & 0.173032 \\
\hline $\mathrm{C}$ & 4.645720 & 1.172642 & -1.516260 \\
\hline $\mathrm{H}$ & 4.477945 & 0.161606 & -1.892673 \\
\hline $\mathrm{H}$ & 5.681149 & 1.485159 & -1.677126 \\
\hline $\mathrm{H}$ & 3.964907 & 1.881510 & -2.000832 \\
\hline $\mathrm{Pd}$ & -0.591573 & 0.325763 & -0.673259 \\
\hline $\mathrm{C}$ & -1.341808 & 1.614267 & -2.094414 \\
\hline $\mathrm{C}$ & -0.960718 & 1.285161 & -3.326339 \\
\hline $\mathrm{H}$ & -0.221449 & 0.505472 & -3.537291 \\
\hline $\mathrm{C}$ & -2.389863 & 2.639872 & -1.827753 \\
\hline $\mathrm{H}$ & -3.062913 & 2.336961 & -1.010094 \\
\hline $\mathrm{H}$ & -1.934090 & 3.594496 & -1.520696 \\
\hline C & -3.192569 & 2.845994 & -3.114875 \\
\hline $\mathrm{H}$ & -3.852026 & 3.716665 & -3.030181 \\
\hline $\mathrm{H}$ & -3.811539 & 1.951361 & -3.312082 \\
\hline O & -2.320186 & 3.091267 & -4.188231 \\
\hline $\mathrm{C}$ & -1.585453 & 1.954722 & -4.533134 \\
\hline $\mathrm{H}$ & -2.222045 & 1.216628 & -5.059808 \\
\hline $\mathrm{H}$ & -0.810284 & 2.278803 & -5.240063 \\
\hline $\mathrm{O}$ & -3.028125 & -1.216595 & 0.398201 \\
\hline B & -2.887750 & -0.863215 & -0.902511 \\
\hline $\mathrm{O}$ & -4.019884 & -0.568543 & -1.564710 \\
\hline $\mathrm{C}$ & -5.088258 & -0.603066 & -0.560354 \\
\hline $\mathrm{C}$ & -4.458390 & -1.483114 & 0.565832 \\
\hline C & -4.626305 & -2.976739 & 0.346256 \\
\hline $\mathrm{H}$ & -4.337525 & -3.273316 & -0.671029 \\
\hline $\mathrm{H}$ & -5.660781 & -3.295877 & 0.521727 \\
\hline $\mathrm{H}$ & -3.974696 & -3.512802 & 1.048863 \\
\hline C & -4.856537 & -1.106207 & 1.975004 \\
\hline $\mathrm{H}$ & -4.559771 & -0.079125 & 2.220910 \\
\hline $\mathrm{H}$ & -4.378413 & -1.787376 & 2.690982 \\
\hline $\mathrm{H}$ & -5.942964 & -1.193892 & 2.107685 \\
\hline $\mathrm{C}$ & -6.329757 & -1.182094 & -1.197756 \\
\hline $\mathrm{H}$ & -6.138457 & -2.157064 & -1.657042 \\
\hline $\mathrm{H}$ & -6.697816 & -0.506087 & -1.978272 \\
\hline $\mathrm{H}$ & -7.125958 & -1.297492 & -0.449940 \\
\hline $\mathrm{C}$ & -5.323094 & 0.835781 & -0.135421 \\
\hline $\mathrm{H}$ & -4.433882 & 1.269188 & 0.344915 \\
\hline $\mathrm{H}$ & -6.164289 & 0.913315 & 0.564695 \\
\hline $\mathrm{H}$ & -5.565732 & 1.437953 & -1.020078 \\
\hline $\mathrm{H}$ & -1.814351 & -0.929373 & -1.496052 \\
\hline $\mathrm{H}$ & 5.058403 & 0.630405 & 0.426464 \\
\hline
\end{tabular}

SCF energy: -3329.873690910

M06-D3 (Gas Phase)/6-31G(d)+SDD (Pd, Fe) opt. No imaginary frequency 


\begin{tabular}{|c|c|c|c|}
\hline \multicolumn{4}{|c|}{ Int-5STania_conf5 } \\
\hline $\mathrm{P}$ & 0.174410 & 2.233859 & 0.362250 \\
\hline $\mathrm{C}$ & -0.391102 & -3.226629 & 0.632280 \\
\hline $\mathrm{C}$ & -1.590481 & -5.749148 & 0.388227 \\
\hline $\mathrm{C}$ & -0.842450 & -3.687281 & -0.609260 \\
\hline C & -0.553459 & -4.048484 & 1.752760 \\
\hline $\mathrm{C}$ & -1.145916 & -5.302259 & 1.627752 \\
\hline $\mathrm{C}$ & -1.434803 & -4.938736 & -0.734055 \\
\hline $\mathrm{H}$ & -0.734964 & -3.067610 & -1.497349 \\
\hline $\mathrm{H}$ & -0.223355 & -3.712898 & 2.734813 \\
\hline $\mathrm{H}$ & -1.264244 & -5.929089 & 2.509243 \\
\hline $\mathrm{H}$ & -1.781485 & -5.277387 & -1.709079 \\
\hline $\mathrm{H}$ & -2.058829 & -6.726762 & 0.295222 \\
\hline $\mathrm{C}$ & 1.183534 & 3.355797 & -0.683759 \\
\hline C & 2.799421 & 5.024772 & -2.248538 \\
\hline $\mathrm{C}$ & 2.104098 & 4.228795 & -0.076158 \\
\hline C & 1.071637 & 3.347965 & -2.076629 \\
\hline C & 1.874073 & 4.179993 & -2.854819 \\
\hline $\mathrm{C}$ & 2.910598 & 5.052741 & -0.857805 \\
\hline $\mathrm{H}$ & 0.348102 & 2.687884 & -2.549092 \\
\hline $\mathrm{H}$ & 1.772357 & 4.167840 & -3.938403 \\
\hline $\mathrm{H}$ & 3.614296 & 5.731045 & -0.378826 \\
\hline $\mathrm{H}$ & 3.425949 & 5.674077 & -2.856671 \\
\hline C & -1.123989 & 3.259654 & 1.101038 \\
\hline $\mathrm{C}$ & -3.258811 & 4.717745 & 2.137877 \\
\hline C & -1.281680 & 4.605167 & 0.759676 \\
\hline $\mathrm{C}$ & -2.046847 & 2.642424 & 1.956410 \\
\hline C & -3.103867 & 3.375216 & 2.480191 \\
\hline $\mathrm{C}$ & -2.350511 & 5.329529 & 1.279798 \\
\hline $\mathrm{H}$ & -0.584499 & 5.089587 & 0.077306 \\
\hline $\mathrm{H}$ & -1.934306 & 1.586349 & 2.211564 \\
\hline $\mathrm{H}$ & -3.814301 & 2.897560 & 3.152440 \\
\hline $\mathrm{H}$ & -2.473619 & 6.376214 & 1.010316 \\
\hline $\mathrm{H}$ & -4.093176 & 5.287698 & 2.541056 \\
\hline C & 1.365604 & 1.866402 & 1.718029 \\
\hline $\mathrm{C}$ & 3.135595 & 1.274998 & 3.809788 \\
\hline C & 1.128282 & 2.338930 & 3.011349 \\
\hline $\mathrm{C}$ & 2.516761 & 1.091465 & 1.471876 \\
\hline $\mathrm{C}$ & 3.373974 & 0.786992 & 2.530622 \\
\hline $\mathrm{C}$ & 2.011085 & 2.054728 & 4.048475 \\
\hline $\mathrm{H}$ & 0.242070 & 2.936348 & 3.216612 \\
\hline $\mathrm{H}$ & 4.230909 & 0.135069 & 2.354326 \\
\hline $\mathrm{H}$ & 1.807170 & 2.433987 & 5.047446 \\
\hline $\mathrm{H}$ & 3.818819 & 1.030646 & 4.620388 \\
\hline C & 0.206950 & -1.253122 & 2.589895 \\
\hline $\mathrm{C}$ & -0.255969 & -0.885948 & 5.317963 \\
\hline $\mathrm{C}$ & -1.045107 & -0.816675 & 3.042016 \\
\hline C & 1.217231 & -1.522441 & 3.513477 \\
\hline C & 0.983551 & -1.336255 & 4.874385 \\
\hline C & -1.273219 & -0.630866 & 4.399822 \\
\hline $\mathrm{H}$ & -1.851838 & -0.664671 & 2.320885 \\
\hline $\mathrm{H}$ & 2.186063 & -1.892488 & 3.174847 \\
\hline $\mathrm{H}$ & 1.773265 & -1.553142 & 5.591893 \\
\hline $\mathrm{H}$ & -2.251765 & -0.298802 & 4.744869 \\
\hline $\mathrm{H}$ & -0.435187 & -0.747916 & 6.382360 \\
\hline $\mathrm{H}$ & 2.188651 & 4.265197 & 1.011267 \\
\hline $\mathrm{H}$ & 2.281081 & -4.079899 & 0.817060 \\
\hline C & 2.783725 & -3.143171 & 0.594284 \\
\hline $\mathrm{C}$ & 2.139823 & -1.869253 & 0.495450 \\
\hline $\mathrm{H}$ & 4.890298 & -3.779136 & 0.199741 \\
\hline $\mathrm{C}$ & 3.151662 & -0.907106 & 0.100087 \\
\hline C & 4.389590 & -1.612129 & -0.024077 \\
\hline $\mathrm{H}$ & 5.347876 & -1.212199 & -0.342632 \\
\hline C & 4.154737 & -2.984662 & 0.268938 \\
\hline $\mathrm{Fe}$ & 2.974521 & -2.380768 & -1.285049 \\
\hline $\mathrm{H}$ & 0.543234 & -2.030902 & -2.697771 \\
\hline $\mathrm{C}$ & 1.552910 & -2.425865 & -2.767907 \\
\hline $\mathrm{C}$ & 1.985464 & -3.742803 & -2.437944 \\
\hline $\mathrm{H}$ & 2. 665517 & -0.664362 & -3.558450 \\
\hline $\mathrm{H}$ & 1.368425 & -4.535765 & -2.026533 \\
\hline C & 3.386756 & -3.813663 & -2.677807 \\
\hline $\mathrm{H}$ & 4.021836 & -4.671694 & -2.484406 \\
\hline C & 3.821846 & -2.542335 & -3.146417 \\
\hline
\end{tabular}

\begin{tabular}{|c|c|c|c|}
\hline $\mathrm{H}$ & 4.843758 & -2.273727 & -3.395946 \\
\hline $\mathrm{C}$ & 2.683852 & -1.689672 & -3.206981 \\
\hline $\mathrm{C}$ & 2.817990 & 0.553009 & 0.089423 \\
\hline $\mathrm{H}$ & 1.920760 & 0.659891 & -0.541567 \\
\hline $\mathrm{N}$ & 3.815298 & 1.492348 & -0.617213 \\
\hline $\mathrm{C}$ & 4.001034 & 1.172352 & -2.060002 \\
\hline $\mathrm{H}$ & 3.014286 & 1.149393 & -2.531457 \\
\hline $\mathrm{H}$ & 4.486206 & 0.198130 & -2.156587 \\
\hline $\mathrm{H}$ & 4.609215 & 1.960374 & -2.513470 \\
\hline $\mathrm{C}$ & 5.122982 & 1.739403 & 0.058987 \\
\hline $\mathrm{H}$ & 4.941370 & 2.098447 & 1.073962 \\
\hline $\mathrm{H}$ & 5.654471 & 2.505334 & -0.513444 \\
\hline $\mathrm{H}$ & 5.710046 & 0.819183 & 0.081521 \\
\hline $\mathrm{Pd}$ & -0.695072 & 0.276465 & -0.568948 \\
\hline $\mathrm{H}$ & 3.319097 & 2.399153 & -0.591017 \\
\hline $\mathrm{P}$ & 0.367422 & -1.557121 & 0.795423 \\
\hline $\mathrm{C}$ & -1.708299 & 1.475612 & -1.889289 \\
\hline C & -2.683296 & 2.329836 & -1.592349 \\
\hline $\mathrm{H}$ & -2.895293 & 2.660072 & -0.573917 \\
\hline $\mathrm{C}$ & -1.439091 & 1.046317 & -3.304813 \\
\hline $\mathrm{H}$ & -0.360735 & 0.957105 & -3.523952 \\
\hline $\mathrm{H}$ & -1.873609 & 0.050657 & -3.488749 \\
\hline C & -2.085795 & 2.063780 & -4.250689 \\
\hline $\mathrm{H}$ & -2.068839 & 1.702409 & -5.284688 \\
\hline $\mathrm{H}$ & -1.535338 & 3.025142 & -4.210230 \\
\hline $\mathrm{O}$ & -3.428194 & 2.250440 & -3.901662 \\
\hline $\mathrm{C}$ & -3.576155 & 2.893889 & -2.668569 \\
\hline $\mathrm{H}$ & -3.378934 & 3.981074 & -2.766159 \\
\hline $\mathrm{H}$ & -4.630204 & 2.784379 & -2.379269 \\
\hline O & -3.062631 & -1.224373 & 0.368555 \\
\hline B & -2.811264 & -1.136106 & -0.958258 \\
\hline O & -3.869265 & -1.197446 & -1.780776 \\
\hline $\mathrm{C}$ & -4.991036 & -1.613371 & -0.931552 \\
\hline C & -4.521058 & -1.164441 & 0.490302 \\
\hline C & -4.931329 & -2.078828 & 1.621915 \\
\hline $\mathrm{H}$ & -4.535918 & -3.091453 & 1.489025 \\
\hline $\mathrm{H}$ & -6.025319 & -2.134978 & 1.699117 \\
\hline $\mathrm{H}$ & -4.546676 & -1.688154 & 2.573210 \\
\hline C & -4.854124 & 0.280689 & 0.816476 \\
\hline $\mathrm{H}$ & -4.540417 & 0.961261 & 0.012484 \\
\hline $\mathrm{H}$ & -4.318913 & 0.566450 & 1.732842 \\
\hline $\mathrm{H}$ & -5.927707 & 0.418111 & 0.992727 \\
\hline $\mathrm{C}$ & -5.092708 & -3.120772 & -1.081278 \\
\hline $\mathrm{H}$ & -4.183818 & -3.625713 & -0.724521 \\
\hline $\mathrm{H}$ & -5.224576 & -3.364687 & -2.141720 \\
\hline $\mathrm{H}$ & -5.952161 & -3.521318 & -0.530159 \\
\hline $\mathrm{C}$ & -6.244740 & -0.933811 & -1.430564 \\
\hline $\mathrm{H}$ & -6.124380 & 0.153100 & -1.492271 \\
\hline $\mathrm{H}$ & -7.092389 & -1.158093 & -0.768941 \\
\hline $\mathrm{H}$ & -6.492800 & -1.302450 & -2.432345 \\
\hline $\mathrm{H}$ & -1.670738 & -1.122534 & -1.429048 \\
\hline
\end{tabular}

SCF energy: -3329.874390030

M06-D3 (Gas Phase)/6-31G(d)+SDD (Pd, Fe) opt. No imaginary frequency 


\begin{tabular}{|c|c|c|c|}
\hline \multicolumn{4}{|c|}{ Int-5STania_confl } \\
\hline $\mathrm{P}$ & 0.314631 & -1.634620 & 0.699860 \\
\hline $\mathrm{P}$ & 0.232215 & 2.125585 & 0.457024 \\
\hline C & -0.472214 & -3.271723 & 0.386061 \\
\hline $\mathrm{C}$ & -1.685286 & -5.757957 & -0.081735 \\
\hline $\mathrm{C}$ & -0.910872 & -3.625479 & -0.895302 \\
\hline C & -0.649419 & -4.186690 & 1.430743 \\
\hline C & -1.249953 & -5.420450 & 1.195362 \\
\hline C & -1.511768 & -4.857552 & -1.129893 \\
\hline $\mathrm{H}$ & -0.786492 & -2.939111 & -1.730399 \\
\hline $\mathrm{H}$ & -0.323532 & -3.940368 & 2.440308 \\
\hline $\mathrm{H}$ & -1.380585 & -6.118597 & 2.019644 \\
\hline $\mathrm{H}$ & -1.849857 & -5.110301 & -2.133237 \\
\hline $\mathrm{H}$ & -2.160028 & -6.720299 & -0.261156 \\
\hline C & 1.158547 & 3.392227 & -0.474429 \\
\hline C & 2.598013 & 5.329045 & -1.882314 \\
\hline $\mathrm{C}$ & 1.689978 & 4.503210 & 0.195445 \\
\hline C & 1.364868 & 3.256760 & -1.849409 \\
\hline C & 2.087449 & 4.219439 & -2.551053 \\
\hline C & 2.397165 & 5.471665 & -0.509507 \\
\hline $\mathrm{H}$ & 0.948506 & 2.395329 & -2.370841 \\
\hline $\mathrm{H}$ & 2.233891 & 4.112146 & -3.624481 \\
\hline $\mathrm{H}$ & 2.792793 & 6.340440 & 0.012775 \\
\hline $\mathrm{H}$ & 3.149334 & 6.089398 & -2.431677 \\
\hline $\mathrm{C}$ & -1.173047 & 2.971828 & 1.246198 \\
\hline C & -3.483972 & 4.106125 & 2.328004 \\
\hline C & -1.445130 & 4.329180 & 1.055046 \\
\hline C & -2.078530 & 2.181376 & 1.967988 \\
\hline C & -3.220551 & 2.750188 & 2.515794 \\
\hline C & -2.598421 & 4.890721 & 1.596724 \\
\hline $\mathrm{H}$ & -0.777595 & 4.951707 & 0.462329 \\
\hline $\mathrm{H}$ & -1.890127 & 1.115357 & 2.097982 \\
\hline $\mathrm{H}$ & -3.908196 & 2.131408 & 3.090237 \\
\hline $\mathrm{H}$ & -2.805778 & 5.947015 & 1.439672 \\
\hline $\mathrm{H}$ & -4.383457 & 4.549856 & 2.749507 \\
\hline C & 1.389677 & 1.696492 & 1.835994 \\
\hline C & 3.033498 & 0.989431 & 4.006144 \\
\hline C & 1.072973 & 2.087683 & 3.141941 \\
\hline C & 2.558393 & 0.937701 & 1.626982 \\
\hline C & 3.351422 & 0.578450 & 2.718420 \\
\hline C & 1.889877 & 1.750455 & 4.215475 \\
\hline $\mathrm{H}$ & 0.169611 & 2.665561 & 3.327305 \\
\hline $\mathrm{H}$ & 4.230592 & -0.051545 & 2.561511 \\
\hline $\mathrm{H}$ & 1.620344 & 2.072939 & 5.218744 \\
\hline $\mathrm{H}$ & 3.667602 & 0.703110 & 4.842440 \\
\hline C & 0.166647 & -1.509848 & 2.516315 \\
\hline C & -0.256229 & -1.426915 & 5.274359 \\
\hline C & -1.091647 & -1.169666 & 3.028709 \\
\hline C & 1.202878 & -1.831017 & 3.393585 \\
\hline C & 0.988938 & -1.787603 & 4.769479 \\
\hline C & -1.299103 & -1.121750 & 4.401333 \\
\hline $\mathrm{H}$ & -1.915664 & -0.979700 & 2.338010 \\
\hline $\mathrm{H}$ & 2.176422 & -2.134124 & 3.006346 \\
\hline $\mathrm{H}$ & 1.798502 & -2.047395 & 5.449709 \\
\hline $\mathrm{H}$ & -2.281259 & -0.859741 & 4.792417 \\
\hline $\mathrm{H}$ & -0.419866 & -1.399036 & 6.349771 \\
\hline $\mathrm{H}$ & 1.551788 & 4.611468 & 1.272644 \\
\hline $\mathrm{H}$ & 2.095495 & -4.194745 & 0.387467 \\
\hline C & 2.654312 & -3.267062 & 0.308687 \\
\hline C & 2.080997 & -1.957919 & 0.390521 \\
\hline $\mathrm{H}$ & 4.730749 & -3.961851 & -0.141149 \\
\hline C & 3.145646 & -1.005860 & 0.146842 \\
\hline C & 4.349515 & -1.762497 & -0.057945 \\
\hline $\mathrm{H}$ & 5.341121 & -1.388067 & -0.304203 \\
\hline $\mathrm{C}$ & 4.036966 & -3.145783 & 0.029799 \\
\hline $\mathrm{Fe}$ & 2.924232 & -2.237222 & -1.434084 \\
\hline $\mathrm{H}$ & 0.478049 & -1.618054 & -2.691712 \\
\hline $\mathrm{C}$ & 1.483572 & -1.990893 & -2.869804 \\
\hline $\mathrm{C}$ & 1.915765 & -3.347620 & -2.818959 \\
\hline $\mathrm{H}$ & 2.591942 & -0.097575 & -3.275572 \\
\hline $\mathrm{H}$ & 1.301598 & -4.206489 & -2.566751 \\
\hline C & 3.312623 & -3.370464 & -3.089835 \\
\hline $\mathrm{H}$ & 3.947071 & -4.250260 & -3.088238 \\
\hline
\end{tabular}

\begin{tabular}{|c|c|c|c|}
\hline $\mathrm{C}$ & 3.744587 & -2.031246 & -3.302282 \\
\hline $\mathrm{H}$ & 4.763867 & -1.724638 & -3.517774 \\
\hline $\mathrm{C}$ & 2.610108 & -1.178869 & -3.166516 \\
\hline $\mathrm{C}$ & 3.006715 & 0.494544 & 0.253953 \\
\hline $\mathrm{H}$ & 2.321556 & 0.871459 & -0.519098 \\
\hline $\mathrm{N}$ & 4.362874 & 1.155004 & -0.093805 \\
\hline $\mathrm{C}$ & 4.534156 & 2.568361 & 0.370709 \\
\hline $\mathrm{H}$ & 4.400753 & 2.627083 & 1.451866 \\
\hline $\mathrm{H}$ & 3.793200 & 3.192304 & -0.135000 \\
\hline $\mathrm{H}$ & 5.543109 & 2.887925 & 0.096323 \\
\hline $\mathrm{C}$ & 4.653043 & 1.092973 & -1.560427 \\
\hline $\mathrm{H}$ & 4.491691 & 0.073275 & -1.915662 \\
\hline $\mathrm{H}$ & 5.685783 & 1.409770 & -1.730085 \\
\hline $\mathrm{H}$ & 3.966145 & 1.786441 & -2.058407 \\
\hline $\mathrm{Pd}$ & -0.617506 & 0.256400 & -0.627766 \\
\hline $\mathrm{C}$ & -1.512852 & 1.550429 & -1.948707 \\
\hline $\mathrm{C}$ & -2.465782 & 2.435536 & -1.671509 \\
\hline $\mathrm{H}$ & -2.739733 & 2.713872 & -0.652837 \\
\hline $\mathrm{C}$ & -1.188053 & 1.165760 & -3.364764 \\
\hline $\mathrm{H}$ & -0.108491 & 1.016849 & -3.534640 \\
\hline $\mathrm{H}$ & -1.674288 & 0.207925 & -3.614126 \\
\hline C & -1.710758 & 2.260326 & -4.300515 \\
\hline $\mathrm{H}$ & -1.653538 & 1.940919 & -5.346874 \\
\hline $\mathrm{H}$ & -1.100577 & 3.177679 & -4.181149 \\
\hline O & -3.057094 & 2.525347 & -4.022939 \\
\hline $\mathrm{C}$ & -3.229778 & 3.128337 & -2.772492 \\
\hline $\mathrm{H}$ & -2.920233 & 4.192999 & -2.805863 \\
\hline $\mathrm{H}$ & -4.306460 & 3.114407 & -2.558780 \\
\hline $\mathrm{O}$ & -3.080365 & -0.928856 & 0.333126 \\
\hline B & -2.828119 & -0.982277 & -0.997829 \\
\hline $\mathrm{O}$ & -3.886638 & -1.133597 & -1.806704 \\
\hline $\mathrm{C}$ & -5.060065 & -0.972753 & -0.940669 \\
\hline $\mathrm{C}$ & -4.484770 & -1.333961 & 0.465173 \\
\hline C & -4.457102 & -2.824682 & 0.755245 \\
\hline $\mathrm{H}$ & -4.006546 & -3.391358 & -0.071446 \\
\hline $\mathrm{H}$ & -5.464244 & -3.217506 & 0.939741 \\
\hline $\mathrm{H}$ & -3.849820 & -3.003508 & 1.652800 \\
\hline C & -5.092241 & -0.569592 & 1.618818 \\
\hline $\mathrm{H}$ & -4.969273 & 0.512806 & 1.498490 \\
\hline $\mathrm{H}$ & -4.612355 & -0.872171 & 2.559974 \\
\hline $\mathrm{H}$ & -6.164073 & -0.790984 & 1.708140 \\
\hline $\mathrm{C}$ & -6.152355 & -1.891766 & -1.434234 \\
\hline $\mathrm{H}$ & -5.810260 & -2.928582 & -1.513996 \\
\hline $\mathrm{H}$ & -6.490420 & -1.568309 & -2.425445 \\
\hline $\mathrm{H}$ & -7.015858 & -1.858288 & -0.756156 \\
\hline $\mathrm{C}$ & -5.477218 & 0.482026 & -1.065585 \\
\hline $\mathrm{H}$ & -4.702446 & 1.160172 & -0.678352 \\
\hline $\mathrm{H}$ & -6.413220 & 0.677387 & -0.527987 \\
\hline $\mathrm{H}$ & -5.634819 & 0.712962 & -2.126316 \\
\hline $\mathrm{H}$ & -1.698514 & -1.028687 & -1.494145 \\
\hline $\mathrm{H}$ & 5.071739 & 0.592046 & 0.391819 \\
\hline
\end{tabular}

SCF energy: -3329.877085940

M06-D3 (Gas Phase) /6-31G(d)+SDD (Pd, Fe) opt. No imaginary frequency 


\begin{tabular}{|c|c|c|c|}
\hline \multicolumn{4}{|c|}{ TS-2STania_conf8 } \\
\hline $\mathrm{P}$ & -0.711791 & -0.023104 & 1.897277 \\
\hline $\mathrm{C}$ & 4.697375 & 0.202292 & 1.418075 \\
\hline $\mathrm{C}$ & 7.374221 & -0.622904 & 1.529780 \\
\hline $\mathrm{C}$ & 5.105242 & -0.986765 & 0.803868 \\
\hline $\mathrm{C}$ & 5.653263 & 0.971354 & 2.093782 \\
\hline $\mathrm{C}$ & 6.981305 & 0.560506 & 2.146742 \\
\hline $\mathrm{C}$ & 6.433247 & -1.395920 & 0.855029 \\
\hline $\mathrm{H}$ & 4.387398 & -1.619024 & 0.284092 \\
\hline $\mathrm{H}$ & 5.361960 & 1.893159 & 2.596348 \\
\hline $\mathrm{H}$ & 7.709565 & 1.166783 & 2.681329 \\
\hline $\mathrm{H}$ & 6.728158 & -2.327845 & 0.376294 \\
\hline $\mathrm{H}$ & 8.411776 & -0.946362 & 1.579811 \\
\hline $\mathrm{C}$ & -2.094727 & -0.136186 & 0.696838 \\
\hline C & -4.218645 & -0.312492 & -1.121164 \\
\hline $\mathrm{C}$ & -3.044008 & 0.896856 & 0.608787 \\
\hline C & -2.247871 & -1.271939 & -0.104900 \\
\hline C & -3.307188 & -1.358424 & -1.005859 \\
\hline C & -4.092219 & 0.812347 & -0.306808 \\
\hline $\mathrm{H}$ & -1.560556 & -2.109673 & -0.003350 \\
\hline $\mathrm{H}$ & -3.420395 & -2.254320 & -1.613007 \\
\hline $\mathrm{H}$ & -4.827648 & 1.613229 & -0.362124 \\
\hline $\mathrm{H}$ & -5.043100 & -0.382263 & -1.827670 \\
\hline $\mathrm{C}$ & -1.374269 & -0.695310 & 3.449723 \\
\hline $\mathrm{C}$ & -2.247017 & -1.941287 & 5.785625 \\
\hline C & -2.675723 & -1.199828 & 3.529802 \\
\hline $\mathrm{C}$ & -0.510003 & -0.820013 & 4.545879 \\
\hline C & -0.950407 & -1.434400 & 5.711000 \\
\hline C & -3.106945 & -1.821780 & 4.698152 \\
\hline $\mathrm{H}$ & -3.354470 & -1.123451 & 2.681198 \\
\hline $\mathrm{H}$ & 0.513651 & -0.449565 & 4.482800 \\
\hline $\mathrm{H}$ & -0.276647 & -1.526339 & 6.560733 \\
\hline $\mathrm{H}$ & -4.119937 & -2.214231 & 4.756958 \\
\hline $\mathrm{H}$ & -2.587406 & -2.431183 & 6.695509 \\
\hline C & -0.538923 & 1.791892 & 2.138043 \\
\hline $\mathrm{C}$ & -0.101340 & 4.523321 & 2.589545 \\
\hline C & -0.856016 & 2.372141 & 3.368305 \\
\hline $\mathrm{C}$ & -0.007519 & 2.602214 & 1.113905 \\
\hline $\mathrm{C}$ & 0.229028 & 3.954772 & 1.364580 \\
\hline $\mathrm{C}$ & -0.650181 & 3.730587 & 3.589841 \\
\hline $\mathrm{H}$ & -1.262397 & 1.755590 & 4.168255 \\
\hline $\mathrm{H}$ & 0.702826 & 4.567551 & 0.595748 \\
\hline $\mathrm{H}$ & -0.905528 & 4.163401 & 4.554642 \\
\hline $\mathrm{H}$ & 0.085342 & 5.580626 & 2.765358 \\
\hline $\mathrm{C}$ & 2.838589 & 1.709764 & 2.942649 \\
\hline $\mathrm{C}$ & 2.807611 & 2.985598 & 5.420720 \\
\hline $\mathrm{C}$ & 2.712832 & 0.959495 & 4.118989 \\
\hline C & 2.967366 & 3.096638 & 3.014391 \\
\hline $\mathrm{C}$ & 2.949827 & 3.731139 & 4.254577 \\
\hline C & 2.693157 & 1.597858 & 5.352634 \\
\hline $\mathrm{H}$ & 2.651180 & -0.132185 & 4.065641 \\
\hline $\mathrm{H}$ & 3.095824 & 3.683017 & 2.103200 \\
\hline $\mathrm{H}$ & 3.055837 & 4.813351 & 4.308880 \\
\hline $\mathrm{H}$ & 2.598982 & 1.010516 & 6.265098 \\
\hline $\mathrm{H}$ & 2.798969 & 3.485541 & 6.387152 \\
\hline $\mathrm{H}$ & -2.977500 & 1.759734 & 1.275396 \\
\hline $\mathrm{H}$ & 5.043583 & 2.374592 & -0.329474 \\
\hline C & 4.010279 & 2.548655 & -0.613957 \\
\hline C & 2.870640 & 1.945517 & 0.009459 \\
\hline $\mathrm{H}$ & 4.195811 & 3.868090 & -2.407733 \\
\hline $\mathrm{C}$ & 1.696311 & 2.377301 & -0.727603 \\
\hline C & 2.146390 & 3.238114 & -1.777295 \\
\hline $\mathrm{H}$ & 1.545163 & 3.707982 & -2.549485 \\
\hline C & 3.564288 & 3.331264 & -1.707930 \\
\hline $\mathrm{Fe}$ & 2.987819 & 1.382309 & -1.931613 \\
\hline $\mathrm{H}$ & 3.032046 & -1.349136 & -1.212887 \\
\hline C & 3.153653 & -0.661062 & -2.044281 \\
\hline $\mathrm{C}$ & 4.359336 & -0.031921 & -2.467263 \\
\hline $\mathrm{H}$ & 1.068857 & -0.536051 & -2.813901 \\
\hline $\mathrm{H}$ & 5.332980 & -0.133859 & -1.997818 \\
\hline C & 4.046909 & 0.803465 & -3.577018 \\
\hline $\mathrm{H}$ & 4.742440 & 1.448401 & -4.103419 \\
\hline C & 2.650497 & 0.697763 & -3.833125 \\
\hline
\end{tabular}

\begin{tabular}{|c|c|c|c|}
\hline $\mathrm{H}$ & 2.108137 & 1.230158 & -4.608596 \\
\hline $\mathrm{C}$ & 2.102115 & -0.212555 & -2.884547 \\
\hline C & 0.324725 & 2.015727 & -0.241183 \\
\hline $\mathrm{H}$ & 0.298636 & 0.917408 & -0.168068 \\
\hline $\mathrm{N}$ & -0.846466 & 2.292422 & -1.203511 \\
\hline C & -0.786638 & 1.486602 & -2.456363 \\
\hline $\mathrm{H}$ & -0.739346 & 0.429477 & -2.177567 \\
\hline $\mathrm{H}$ & 0.100669 & 1.767604 & -3.028727 \\
\hline $\mathrm{H}$ & -1.698330 & 1.672937 & -3.031296 \\
\hline $\mathrm{C}$ & -1.185677 & 3.719059 & -1.482875 \\
\hline $\mathrm{H}$ & -1.339083 & 4.244521 & -0.538486 \\
\hline $\mathrm{H}$ & -2.110735 & 3.734087 & -2.066247 \\
\hline $\mathrm{H}$ & -0.383150 & 4.189961 & -2.052677 \\
\hline $\mathrm{Pd}$ & 1.361071 & -1.082624 & 1.387633 \\
\hline $\mathrm{H}$ & -1.654979 & 1.911509 & -0.679952 \\
\hline $\mathrm{P}$ & 2.944983 & 0.764007 & 1.388990 \\
\hline $\mathrm{C}$ & 0.450809 & -2.976720 & 1.287287 \\
\hline C & -0.397524 & -3.505205 & 2.177924 \\
\hline $\mathrm{H}$ & -0.465653 & -3.130672 & 3.201721 \\
\hline C & 0.626545 & -3.577866 & -0.083149 \\
\hline $\mathrm{H}$ & 0.208464 & -2.919981 & -0.865440 \\
\hline $\mathrm{H}$ & 1.694630 & -3.693227 & -0.322632 \\
\hline $\mathrm{C}$ & -0.050627 & -4.945835 & -0.118902 \\
\hline $\mathrm{H}$ & 0.557699 & -5.668795 & 0.455485 \\
\hline $\mathrm{H}$ & -0.152739 & -5.320512 & -1.142675 \\
\hline 0 & -1.348637 & -4.858549 & 0.423630 \\
\hline C & -1.306036 & -4.639953 & 1.806449 \\
\hline $\mathrm{H}$ & -2.330506 & -4.399835 & 2.128380 \\
\hline $\mathrm{H}$ & -1.008231 & -5.561778 & 2.345491 \\
\hline 0 & 2.335818 & -2.545791 & 3.589584 \\
\hline B & 2.375235 & -2.939482 & 2.278257 \\
\hline 0 & 2.998516 & -4.123965 & 2.047635 \\
\hline C & 3.243694 & -4.702792 & 3.368472 \\
\hline $\mathrm{C}$ & 3.256807 & -3.438764 & 4.286096 \\
\hline C & 4.601118 & -2.727571 & 4.317939 \\
\hline $\mathrm{H}$ & 4.991911 & -2.546122 & 3.306485 \\
\hline $\mathrm{H}$ & 5.348413 & -3.300737 & 4.880234 \\
\hline $\mathrm{H}$ & 4.483046 & -1.753338 & 4.811026 \\
\hline C & 2.729976 & -3.652109 & 5.686964 \\
\hline $\mathrm{H}$ & 1.688814 & -3.994779 & 5.681709 \\
\hline $\mathrm{H}$ & 2.774887 & -2.705614 & 6.242502 \\
\hline $\mathrm{H}$ & 3.339683 & -4.386820 & 6.229435 \\
\hline $\mathrm{C}$ & 4.550395 & -5.460705 & 3.317333 \\
\hline $\mathrm{H}$ & 5.368017 & -4.836758 & 2.941004 \\
\hline $\mathrm{H}$ & 4.452585 & -6.328653 & 2.654820 \\
\hline $\mathrm{H}$ & 4.820894 & -5.829082 & 4.316158 \\
\hline $\mathrm{C}$ & 2.089277 & -5.646019 & 3.659354 \\
\hline $\mathrm{H}$ & 1.131876 & -5.112524 & 3.725263 \\
\hline $\mathrm{H}$ & 2.245419 & -6.188532 & 4.599833 \\
\hline $\mathrm{H}$ & 2.017710 & -6.383924 & 2.850787 \\
\hline $\mathrm{H}$ & 2.738504 & -2.104955 & 1.297072 \\
\hline
\end{tabular}

SCF energy: -3329.867816390

M06-D3 (Gas Phase) /6-31G(d)+SDD (Pd, Fe) opt. 1 imaginary frequency: $\quad 207 \mathrm{i} \mathrm{cm-1}$ 


\begin{tabular}{|c|c|c|c|}
\hline \multicolumn{4}{|c|}{ Int-5RTania_conf5 } \\
\hline $\mathrm{P}$ & 0.301484 & -1.498278 & 0.730755 \\
\hline $\mathrm{P}$ & 0.385619 & 2. 270267 & 0.315790 \\
\hline $\mathrm{C}$ & -0.568737 & -3.104598 & 0.487599 \\
\hline C & -2.044013 & -5.460445 & 0.112110 \\
\hline C & -0.966750 & -3.504058 & -0.793185 \\
\hline $\mathrm{C}$ & -0.917836 & -3.906125 & 1.578572 \\
\hline $\mathrm{C}$ & -1.649477 & -5.076322 & 1.388782 \\
\hline $\mathrm{C}$ & -1.694578 & -4.672639 & -0.983212 \\
\hline $\mathrm{H}$ & -0.721737 & -2.892687 & -1.659381 \\
\hline $\mathrm{H}$ & -0.632325 & -3.619955 & 2.589499 \\
\hline $\mathrm{H}$ & -1.914077 & -5.687343 & 2.249466 \\
\hline $\mathrm{H}$ & -1.996575 & -4.964280 & -1.987933 \\
\hline $\mathrm{H}$ & -2.620055 & -6.372197 & -0.032031 \\
\hline $\mathrm{C}$ & 1.393093 & 3.399684 & -0.705577 \\
\hline C & 2.984788 & 5.119625 & -2.225758 \\
\hline C & 1.973232 & 4.532154 & -0.115614 \\
\hline C & 1.623950 & 3.134445 & -2.057431 \\
\hline C & 2.423468 & 3.989486 & -2.813959 \\
\hline $\mathrm{C}$ & 2.756095 & 5.393000 & -0.877142 \\
\hline $\mathrm{H}$ & 1.151736 & 2.269173 & -2.521096 \\
\hline $\mathrm{H}$ & 2.586955 & 3.785079 & -3.870605 \\
\hline $\mathrm{H}$ & 3.188829 & 6.279836 & -0.418470 \\
\hline $\mathrm{H}$ & 3.594600 & 5.796662 & -2.820444 \\
\hline $\mathrm{C}$ & -0.934369 & 3.283612 & 1.054801 \\
\hline $\mathrm{C}$ & -3.138069 & 4.672287 & 2.060660 \\
\hline C & -1.157072 & 4.612173 & 0.682953 \\
\hline C & -1.832595 & 2.651506 & 1.925506 \\
\hline C & -2.921445 & 3.347017 & 2.433724 \\
\hline C & -2.257133 & 5.300918 & 1.187403 \\
\hline $\mathrm{H}$ & -0.493324 & 5.111586 & -0.020423 \\
\hline $\mathrm{H}$ & -1.669083 & 1.611949 & 2.215623 \\
\hline $\mathrm{H}$ & -3.606460 & 2.853493 & 3.121017 \\
\hline $\mathrm{H}$ & -2.425837 & 6.333552 & 0.889780 \\
\hline $\mathrm{H}$ & -3.996133 & 5.214683 & 2.451869 \\
\hline $\mathrm{C}$ & 1.527454 & 1.842146 & 1.712397 \\
\hline C & 3.111344 & 1.107962 & 3.921055 \\
\hline C & 1.244305 & 2.316162 & 2.998250 \\
\hline C & 2.641030 & 0.994960 & 1.542723 \\
\hline $\mathrm{C}$ & 3.401222 & 0.622631 & 2.652836 \\
\hline $\mathrm{C}$ & 2.032153 & 1.966479 & 4.089515 \\
\hline $\mathrm{H}$ & 0.390358 & 2.971658 & 3.155857 \\
\hline $\mathrm{H}$ & 4.233309 & -0.074294 & 2.525066 \\
\hline $\mathrm{H}$ & 1.788230 & 2.356147 & 5.075322 \\
\hline $\mathrm{H}$ & 3.718092 & 0.808162 & 4.772729 \\
\hline $\mathrm{C}$ & 0.199693 & -1.298270 & 2.543209 \\
\hline C & -0.184745 & -1.119921 & 5.302837 \\
\hline $\mathrm{C}$ & -1.009617 & -0.818252 & 3.060021 \\
\hline C & 1.207184 & -1.705600 & 3.418181 \\
\hline C & 1.012850 & -1.613731 & 4.794667 \\
\hline C & -1.199916 & -0.725421 & 4.432794 \\
\hline $\mathrm{H}$ & -1.816579 & -0.563960 & 2.371252 \\
\hline $\mathrm{H}$ & 2.140764 & -2.113870 & 3.028436 \\
\hline $\mathrm{H}$ & 1.798774 & -1.940505 & 5.473577 \\
\hline $\mathrm{H}$ & -2.147344 & -0.357708 & 4.825785 \\
\hline $\mathrm{H}$ & -0.333727 & -1.056673 & 6.378889 \\
\hline $\mathrm{H}$ & 1.812429 & 4.742458 & 0.943152 \\
\hline $\mathrm{H}$ & 1.945175 & -4.146097 & 0.437727 \\
\hline C & 2.549794 & -3.250069 & 0.333932 \\
\hline C & 2.044029 & -1.912581 & 0.396753 \\
\hline $\mathrm{H}$ & 4.585352 & -4.056654 & -0.116474 \\
\hline C & 3.152024 & -1.020337 & 0.126422 \\
\hline C & 4.315298 & -1.839635 & -0.072902 \\
\hline $\mathrm{H}$ & 5.322264 & -1.520355 & -0.333992 \\
\hline C & 3.934395 & -3.203789 & 0.042868 \\
\hline $\mathrm{Fe}$ & 2.860054 & -2.265350 & -1.430961 \\
\hline $\mathrm{H}$ & 0.438193 & -1.555952 & -2.688406 \\
\hline C & 1.427391 & -1.971776 & -2.865287 \\
\hline C & 1.804213 & -3.344962 & -2.806113 \\
\hline $\mathrm{H}$ & 2.612897 & -0.128346 & -3.285433 \\
\hline $\mathrm{H}$ & 1.158050 & -4.177931 & -2.547895 \\
\hline C & 3.198166 & -3.426997 & -3.078929 \\
\hline $\mathrm{H}$ & 3.795758 & -4.332182 & -3.072088 \\
\hline
\end{tabular}

\begin{tabular}{|c|c|c|c|}
\hline C & 3.683989 & -2.108381 & -3.302164 \\
\hline $\mathrm{H}$ & 4.714657 & -1.846068 & -3.522034 \\
\hline C & 2.585936 & -1.208899 & -3.170220 \\
\hline C & 3.082171 & 0.486849 & 0.191303 \\
\hline $\mathrm{H}$ & 2.427921 & 0.874086 & -0.602402 \\
\hline $\mathrm{N}$ & 4.475775 & 1.070304 & -0.155207 \\
\hline C & 4.725936 & 2.476547 & 0.295582 \\
\hline $\mathrm{H}$ & 4.603997 & 2.551679 & 1.376938 \\
\hline $\mathrm{H}$ & 4.017519 & 3.136838 & -0.210562 \\
\hline $\mathrm{H}$ & 5.749092 & 2.737775 & 0.012013 \\
\hline C & 4.770545 & 0.973407 & -1.619121 \\
\hline $\mathrm{H}$ & 4.552071 & -0.039622 & -1.962468 \\
\hline $\mathrm{H}$ & 5.821440 & 1.225747 & -1.785479 \\
\hline $\mathrm{H}$ & 4.128664 & 1.700076 & -2.130112 \\
\hline $\mathrm{Pd}$ & -0.622184 & 0.390829 & -0.624256 \\
\hline C & -1.556519 & 1.673523 & -1.942505 \\
\hline $\mathrm{C}$ & -1.211140 & 1.517796 & -3.218172 \\
\hline $\mathrm{H}$ & -0.361755 & 0.905339 & -3.536974 \\
\hline $\mathrm{C}$ & -2.782774 & 2.424136 & -1.533000 \\
\hline $\mathrm{H}$ & -3.320421 & 1.910612 & -0.719733 \\
\hline $\mathrm{H}$ & -2.532518 & 3.426189 & -1.155443 \\
\hline C & -3.699328 & 2.539427 & -2.754581 \\
\hline $\mathrm{H}$ & -4.521796 & 3.236747 & -2.560386 \\
\hline $\mathrm{H}$ & -4.125489 & 1.546775 & -2.991275 \\
\hline O & -2.977881 & 3.047984 & -3.847040 \\
\hline C & -2.038911 & 2.132407 & -4.326102 \\
\hline $\mathrm{H}$ & -2.533468 & 1.317255 & -4.890960 \\
\hline $\mathrm{H}$ & -1.397424 & 2.675316 & -5.032837 \\
\hline $\mathrm{O}$ & -2.995482 & -1.240855 & 0.456426 \\
\hline B & -2.873241 & -0.929587 & -0.853224 \\
\hline $\mathrm{O}$ & -4.017707 & -0.758643 & -1.536303 \\
\hline $\mathrm{C}$ & -5.072412 & -1.290587 & -0.667119 \\
\hline C & -4.423695 & -1.204262 & 0.757920 \\
\hline C & -4.722637 & -2.371999 & 1.673162 \\
\hline $\mathrm{H}$ & -4.380011 & -3.320455 & 1.244226 \\
\hline $\mathrm{H}$ & -5.798590 & -2.442721 & 1.880871 \\
\hline $\mathrm{H}$ & -4.200516 & -2.231371 & 2.629069 \\
\hline C & -4.679128 & 0.110108 & 1.475471 \\
\hline $\mathrm{H}$ & -4.397582 & 0.978176 & 0.863019 \\
\hline $\mathrm{H}$ & -4.081287 & 0.136712 & 2.397577 \\
\hline $\mathrm{H}$ & -5.733124 & 0.214995 & 1.759733 \\
\hline $\mathrm{C}$ & -5.310707 & -2.715947 & -1.132815 \\
\hline $\mathrm{H}$ & -4.407834 & -3.333655 & -1.021785 \\
\hline $\mathrm{H}$ & -5.584508 & -2.703319 & -2.194035 \\
\hline $\mathrm{H}$ & -6.127015 & -3.190179 & -0.574791 \\
\hline $\mathrm{C}$ & -6.310761 & -0.445455 & -0.856162 \\
\hline $\mathrm{H}$ & -6.112431 & 0.618288 & -0.680800 \\
\hline $\mathrm{H}$ & -7.107713 & -0.770645 & -0.173966 \\
\hline $\mathrm{H}$ & -6.681097 & -0.556236 & -1.881955 \\
\hline $\mathrm{H}$ & -1.792400 & -0.903016 & -1.432848 \\
\hline $\mathrm{H}$ & 5.148844 & 0.474070 & 0.341368 \\
\hline
\end{tabular}

SCF energy: -3329.871970830

M06-D3 (Gas Phase) /6-31G(d)+SDD (Pd, Fe) opt. No imaginary frequency 


\begin{tabular}{|c|c|c|c|}
\hline \multicolumn{4}{|c|}{ Int-5RTania_conf4 } \\
\hline $\mathrm{P}$ & 0.246825 & 2.308527 & 0.278172 \\
\hline $\mathrm{C}$ & -0.464944 & -3.126472 & 0.540468 \\
\hline $\mathrm{C}$ & -1.943624 & -5.480411 & 0.155038 \\
\hline $\mathrm{C}$ & -0.782278 & -3.572134 & -0.748123 \\
\hline C & -0.900684 & -3.880136 & 1.634425 \\
\hline $\mathrm{C}$ & -1.632603 & -5.049240 & 1.439394 \\
\hline $\mathrm{C}$ & -1.510260 & -4.739475 & -0.942940 \\
\hline $\mathrm{H}$ & -0.470824 & -2.998371 & -1.617396 \\
\hline $\mathrm{H}$ & -0.688998 & -3.557041 & 2.651748 \\
\hline $\mathrm{H}$ & -1.963617 & -5.622289 & 2.303338 \\
\hline $\mathrm{H}$ & -1.746392 & -5.066890 & -1.954101 \\
\hline $\mathrm{H}$ & -2.519772 & -6.391512 & 0.006958 \\
\hline C & 1. 342291 & 3.356915 & -0.750993 \\
\hline C & 3.030424 & 4.978742 & -2.284298 \\
\hline $\mathrm{C}$ & 2.262043 & 4.217018 & -0.124748 \\
\hline C & 1.267900 & 3.331463 & -2.146299 \\
\hline C & 2.107588 & 4.143990 & -2.907202 \\
\hline C & 3.105485 & 5.018395 & -0.891380 \\
\hline $\mathrm{H}$ & 0.534367 & 2.688339 & -2.632574 \\
\hline $\mathrm{H}$ & 2.033729 & 4.126822 & -3.992794 \\
\hline $\mathrm{H}$ & 3.805298 & 5.692297 & -0.400300 \\
\hline $\mathrm{H}$ & 3.682158 & 5.613499 & -2.881154 \\
\hline $\mathrm{C}$ & -1.051669 & 3.409987 & 0.905853 \\
\hline C & -3.174402 & 4.991648 & 1.775475 \\
\hline C & -1.080431 & 4.772122 & 0.596389 \\
\hline $\mathrm{C}$ & -2.094154 & 2.842840 & 1.650764 \\
\hline C & -3.145038 & 3.634498 & 2.092890 \\
\hline C & -2.145079 & 5.557284 & 1.029936 \\
\hline $\mathrm{H}$ & -0.287377 & 5.224585 & 0.003034 \\
\hline $\mathrm{H}$ & -2.082197 & 1.774390 & 1.878801 \\
\hline $\mathrm{H}$ & -3.949671 & 3.192097 & 2.677639 \\
\hline $\mathrm{H}$ & -2.169186 & 6.615889 & 0.780881 \\
\hline $\mathrm{H}$ & -4.004875 & 5.609559 & 2.110178 \\
\hline $\mathrm{C}$ & 1.319178 & 1.916583 & 1.725013 \\
\hline C & 2.772926 & 1.136087 & 3.993710 \\
\hline C & 0.963062 & 2.372035 & 2.996076 \\
\hline $\mathrm{C}$ & 2.443320 & 1.074316 & 1.591515 \\
\hline $\mathrm{C}$ & 3.134978 & 0.672097 & 2.733666 \\
\hline $\mathrm{C}$ & 1.694741 & 2.001222 & 4.121037 \\
\hline $\mathrm{H}$ & 0.098690 & 3.022354 & 3.116445 \\
\hline $\mathrm{H}$ & 3.958401 & -0.037501 & 2.637576 \\
\hline $\mathrm{H}$ & 1.400716 & 2.372096 & 5.100470 \\
\hline $\mathrm{H}$ & 3.324874 & 0.809288 & 4.872247 \\
\hline C & 0.286069 & -1.313419 & 2.590157 \\
\hline $\mathrm{C}$ & -0.112016 & -1.247947 & 5.351609 \\
\hline C & -0.861760 & -0.720185 & 3.124559 \\
\hline C & 1. 233112 & -1.876623 & 3.448785 \\
\hline C & 1.033232 & -1.841084 & 4.825655 \\
\hline C & -1.061414 & -0.687878 & 4.499920 \\
\hline $\mathrm{H}$ & -1.621818 & -0.327429 & 2.447089 \\
\hline $\mathrm{H}$ & 2.120250 & -2.364218 & 3.040927 \\
\hline $\mathrm{H}$ & 1.768185 & -2.291036 & 5.490718 \\
\hline $\mathrm{H}$ & -1.963875 & -0.234361 & 4.907671 \\
\hline $\mathrm{H}$ & -0.269928 & -1.232952 & 6.428205 \\
\hline $\mathrm{H}$ & 2.302232 & 4.275231 & 0.965241 \\
\hline $\mathrm{H}$ & 2.151878 & -4.072215 & 0.385073 \\
\hline C & 2.700214 & -3.139146 & 0.294369 \\
\hline C & 2.119239 & -1.835870 & 0.400554 \\
\hline $\mathrm{H}$ & 4.773217 & -3.807713 & -0.210578 \\
\hline C & 3.173584 & -0.872326 & 0.144894 \\
\hline C & 4.375044 & -1.611522 & -0.101553 \\
\hline $\mathrm{H}$ & 5.351779 & -1.219580 & -0.368274 \\
\hline C & 4.075399 & -2.999825 & -0.017870 \\
\hline $\mathrm{Fe}$ & 2.926239 & -2.097867 & -1.448282 \\
\hline $\mathrm{H}$ & 0.438629 & -1.598949 & -2.694709 \\
\hline C & 1.464655 & -1.910170 & -2.875142 \\
\hline $\mathrm{C}$ & 1.976192 & -3.239906 & -2.850901 \\
\hline $\mathrm{H}$ & 2.452138 & 0.051047 & -3.251321 \\
\hline $\mathrm{H}$ & 1.417709 & -4.141027 & -2.618268 \\
\hline C & 3.370624 & -3.174233 & -3.126355 \\
\hline $\mathrm{H}$ & 4.055693 & -4.015096 & -3.142745 \\
\hline $\mathrm{C}$ & 3.722243 & -1.807924 & -3.313618 \\
\hline
\end{tabular}

\begin{tabular}{|c|c|c|c|}
\hline $\mathrm{H}$ & 4.720700 & -1.438412 & -3.527039 \\
\hline $\mathrm{C}$ & 2.540093 & -1.027710 & -3.158012 \\
\hline $\mathrm{C}$ & 2.909173 & 0.604248 & 0.231368 \\
\hline $\mathrm{H}$ & 2.130712 & 0.830522 & -0.512749 \\
\hline $\mathrm{N}$ & 4.065234 & 1.522385 & -0.226818 \\
\hline $\mathrm{C}$ & 4.405413 & 1.379414 & -1.673548 \\
\hline $\mathrm{H}$ & 3.505932 & 1.594168 & -2.256368 \\
\hline $\mathrm{H}$ & 4.751153 & 0.362435 & -1.868992 \\
\hline $\mathrm{H}$ & 5.181353 & 2.109600 & -1.919669 \\
\hline $\mathrm{C}$ & 5.294537 & 1.539772 & 0.623030 \\
\hline $\mathrm{H}$ & 5.025291 & 1.823086 & 1.641643 \\
\hline $\mathrm{H}$ & 5.980975 & 2.280803 & 0.204837 \\
\hline $\mathrm{H}$ & 5.764276 & 0.554932 & 0.617755 \\
\hline $\mathrm{Pd}$ & -0.679026 & 0.348580 & -0.597409 \\
\hline $\mathrm{H}$ & 3.647651 & 2.465326 & -0.141152 \\
\hline $\mathrm{P}$ & 0.365514 & -1.497681 & 0.775888 \\
\hline $\mathrm{C}$ & -1.673646 & 1.510199 & -1.981566 \\
\hline C & -1.347294 & 1.225605 & -3.240450 \\
\hline $\mathrm{H}$ & -0.512034 & 0.569082 & -3.506074 \\
\hline $\mathrm{C}$ & -2.860341 & 2.345519 & -1.630981 \\
\hline $\mathrm{H}$ & -3.413371 & 1.924683 & -0.776302 \\
\hline $\mathrm{H}$ & -2.558468 & 3.362991 & -1.336276 \\
\hline C & -3.782921 & 2.402374 & -2.852726 \\
\hline $\mathrm{H}$ & -4.563916 & 3.158215 & -2.716104 \\
\hline $\mathrm{H}$ & -4.264613 & 1.417534 & -2.996948 \\
\hline $\mathrm{O}$ & -3.049990 & 2.768439 & -3.993685 \\
\hline $\mathrm{C}$ & -2.170842 & 1.760183 & -4.393290 \\
\hline $\mathrm{H}$ & -2.719549 & 0.916892 & -4.857522 \\
\hline $\mathrm{H}$ & -1.521759 & 2.190942 & -5.167101 \\
\hline O & -2.947979 & -1.359416 & 0.572622 \\
\hline B & -2.840379 & -1.077721 & -0.745678 \\
\hline O & -3.993186 & -0.930981 & -1.422110 \\
\hline $\mathrm{C}$ & -5.030088 & -1.459427 & -0.529122 \\
\hline C & -4.373283 & -1.304354 & 0.883901 \\
\hline C & -4.672619 & -2.418528 & 1.862235 \\
\hline $\mathrm{H}$ & -4.350096 & -3.392694 & 1.478867 \\
\hline $\mathrm{H}$ & -5.746348 & -2.463346 & 2.088186 \\
\hline $\mathrm{H}$ & -4.134143 & -2.234089 & 2.801471 \\
\hline C & -4.621622 & 0.048202 & 1.529549 \\
\hline $\mathrm{H}$ & -4.358447 & 0.876838 & 0.856827 \\
\hline $\mathrm{H}$ & -4.005741 & 0.131301 & 2.436555 \\
\hline $\mathrm{H}$ & -5.670615 & 0.166074 & 1.826949 \\
\hline $\mathrm{C}$ & -5.232218 & -2.907718 & -0.938141 \\
\hline $\mathrm{H}$ & -4.314331 & -3.498381 & -0.802838 \\
\hline $\mathrm{H}$ & -5.505995 & -2.943983 & -1.998849 \\
\hline $\mathrm{H}$ & -6.037245 & -3.378802 & -0.361256 \\
\hline $\mathrm{C}$ & -6.291691 & -0.654922 & -0.739653 \\
\hline $\mathrm{H}$ & -6.118542 & 0.420028 & -0.612896 \\
\hline $\mathrm{H}$ & -7.072759 & -0.970063 & -0.034719 \\
\hline $\mathrm{H}$ & -6.670545 & -0.819029 & -1.755172 \\
\hline $\mathrm{H}$ & -1.762104 & -1.061895 & -1.332963 \\
\hline
\end{tabular}

SCF energy: -3329.873014710

M06-D3 (Gas Phase) /6-31G(d)+SDD (Pd, Fe) opt. No imaginary frequency 


\begin{tabular}{|c|c|c|c|}
\hline \multicolumn{4}{|c|}{ Int-5STania_conf 2} \\
\hline $\mathrm{P}$ & 0.338026 & -1.603937 & 0.643744 \\
\hline $\mathrm{P}$ & 0.243646 & 2.165632 & 0.505927 \\
\hline $\mathrm{C}$ & -0.435958 & -3.238225 & 0.289012 \\
\hline C & -1.624745 & -5.723609 & -0.238349 \\
\hline C & -0.870874 & -3.564844 & -1.000622 \\
\hline C & -0.605612 & -4.178969 & 1.312059 \\
\hline C & -1.192633 & -5.412815 & 1.047158 \\
\hline $\mathrm{C}$ & -1.461681 & -4.796344 & -1.264412 \\
\hline $\mathrm{H}$ & -0.753822 & -2.857524 & -1.819305 \\
\hline $\mathrm{H}$ & -0.285623 & -3.950974 & 2.328054 \\
\hline $\mathrm{H}$ & -1.317079 & -6.131814 & 1.854299 \\
\hline $\mathrm{H}$ & -1.799524 & -5.026954 & -2.273090 \\
\hline $\mathrm{H}$ & -2.089926 & -6.686084 & -0.440828 \\
\hline $\mathrm{C}$ & 1.197431 & 3.400719 & -0.438366 \\
\hline C & 2.711796 & 5.259496 & -1.871680 \\
\hline C & 1.652194 & 4.570671 & 0.185258 \\
\hline C & 1.522272 & 3.165172 & -1.776153 \\
\hline C & 2.278825 & 4.090533 & -2.492205 \\
\hline $\mathrm{C}$ & 2.398767 & 5.498563 & -0.533542 \\
\hline $\mathrm{H}$ & 1.170674 & 2.251080 & -2.256369 \\
\hline $\mathrm{H}$ & 2.512113 & 3.908034 & -3.539957 \\
\hline $\mathrm{H}$ & 2.735610 & 6.413090 & -0.049661 \\
\hline $\mathrm{H}$ & 3.289818 & 5.991161 & -2.432367 \\
\hline $\mathrm{C}$ & -1.152835 & 3.043688 & 1.272523 \\
\hline $\mathrm{C}$ & -3.493642 & 4.201024 & 2.262206 \\
\hline C & -1.504183 & 4.344754 & 0.902730 \\
\hline C & -1.996300 & 2.316953 & 2.124738 \\
\hline C & -3.154976 & 2.898034 & 2.623765 \\
\hline C & -2.669604 & 4.919697 & 1.402056 \\
\hline $\mathrm{H}$ & -0.888545 & 4.908379 & 0.203931 \\
\hline $\mathrm{H}$ & -1.745783 & 1.291334 & 2.399874 \\
\hline $\mathrm{H}$ & -3.797285 & 2.329422 & 3.294182 \\
\hline $\mathrm{H}$ & -2.935240 & 5.933649 & 1.110545 \\
\hline $\mathrm{H}$ & -4.403898 & 4.654386 & 2.648936 \\
\hline $\mathrm{C}$ & 1.386777 & 1.724275 & 1.896967 \\
\hline $\mathrm{C}$ & 2.990844 & 0.982520 & 4.089311 \\
\hline $\mathrm{C}$ & 1.070704 & 2.130007 & 3.198870 \\
\hline $\mathrm{C}$ & 2.545222 & 0.944873 & 1.703385 \\
\hline $\mathrm{C}$ & 3.314568 & 0.565540 & 2.805084 \\
\hline $\mathrm{C}$ & 1.867635 & 1.777205 & 4.282328 \\
\hline $\mathrm{H}$ & 0.184345 & 2.735541 & 3.374781 \\
\hline $\mathrm{H}$ & 4.182795 & -0.081562 & 2.657258 \\
\hline $\mathrm{H}$ & 1.597779 & 2.115071 & 5.280427 \\
\hline $\mathrm{H}$ & 3.607122 & 0.680710 & 4.933415 \\
\hline $\mathrm{C}$ & 0.120298 & -1.487283 & 2.453748 \\
\hline $\mathrm{C}$ & -0.416126 & -1.402494 & 5.192176 \\
\hline $\mathrm{C}$ & -1.149987 & -1.119087 & 2.913958 \\
\hline $\mathrm{C}$ & 1.112307 & -1.831347 & 3.372487 \\
\hline $\mathrm{C}$ & 0.841883 & -1.786431 & 4.738477 \\
\hline $\mathrm{C}$ & -1.414341 & -1.072188 & 4.277351 \\
\hline $\mathrm{H}$ & -1.935936 & -0.894558 & 2.189738 \\
\hline $\mathrm{H}$ & 2.095839 & -2.150734 & 3.024922 \\
\hline $\mathrm{H}$ & 1.617505 & -2.062089 & 5.451189 \\
\hline $\mathrm{H}$ & -2.405915 & -0.787511 & 4.627778 \\
\hline $\mathrm{H}$ & -0.623758 & -1.373682 & 6.259925 \\
\hline $\mathrm{H}$ & 1.420250 & 4.758811 & 1.234767 \\
\hline $\mathrm{H}$ & 2.132473 & -4.167502 & 0.360071 \\
\hline $\mathrm{C}$ & 2.690912 & -3.237430 & 0.315085 \\
\hline $\mathrm{C}$ & 2.112809 & -1.930373 & 0.400372 \\
\hline $\mathrm{H}$ & 4.781869 & -3.920642 & -0.082394 \\
\hline $\mathrm{C}$ & 3.182244 & -0.973529 & 0.206985 \\
\hline $\mathrm{C}$ & 4.393459 & -1.723501 & 0.025849 \\
\hline $\mathrm{H}$ & 5.390844 & -1.341741 & -0.182923 \\
\hline $\mathrm{C}$ & 4.081396 & -3.108704 & 0.080692 \\
\hline $\mathrm{Fe}$ & 3.009662 & -2.178924 & -1.400327 \\
\hline $\mathrm{H}$ & 0.588960 & -1.563326 & -2.693993 \\
\hline $\mathrm{C}$ & 1.601068 & -1.920649 & -2.862490 \\
\hline $\mathrm{C}$ & 2.046289 & -3.273535 & -2.827764 \\
\hline $\mathrm{H}$ & 2.695517 & -0.007663 & -3.208188 \\
\hline $\mathrm{H}$ & 1.435133 & -4.142712 & -2.604859 \\
\hline $\mathrm{C}$ & 3.449185 & -3.276106 & -3.067220 \\
\hline $\mathrm{H}$ & 4.093402 & -4.148779 & -3.067768 \\
\hline
\end{tabular}

\begin{tabular}{|c|c|c|c|}
\hline $\mathrm{C}$ & 3.870776 & -1.928355 & -3.244648 \\
\hline $\mathrm{H}$ & 4.891101 & -1.607046 & -3.431884 \\
\hline C & 2.724228 & -1.090536 & -3.118832 \\
\hline C & 3.029243 & 0.522465 & 0.336162 \\
\hline $\mathrm{H}$ & 2.363386 & 0.907128 & -0.448617 \\
\hline $\mathrm{N}$ & 4.389785 & 1.199915 & 0.040660 \\
\hline C & 4.532183 & 2.607460 & 0.534361 \\
\hline $\mathrm{H}$ & 4.397987 & 2.640354 & 1.616455 \\
\hline $\mathrm{H}$ & 3.779092 & 3.227586 & 0.042302 \\
\hline $\mathrm{H}$ & 5.534730 & 2.952406 & 0.267479 \\
\hline $\mathrm{C}$ & 4.729512 & 1.160555 & -1.416356 \\
\hline $\mathrm{H}$ & 4.575280 & 0.147467 & -1.793308 \\
\hline $\mathrm{H}$ & 5.769355 & 1.473491 & -1.544845 \\
\hline $\mathrm{H}$ & 4.064126 & 1.866378 & -1.925948 \\
\hline $\mathrm{Pd}$ & -0.599767 & 0.322508 & -0.626262 \\
\hline C & -1.583563 & 1.672961 & -1.825661 \\
\hline $\mathrm{C}$ & -2.662740 & 2.340555 & -1.425644 \\
\hline $\mathrm{H}$ & -2.986942 & 2.368547 & -0.382965 \\
\hline $\mathrm{C}$ & -1.243671 & 1.523746 & -3.280413 \\
\hline $\mathrm{H}$ & -0.440660 & 2.213305 & -3.581642 \\
\hline $\mathrm{H}$ & -0.897738 & 0.504667 & -3.522416 \\
\hline C & -2.506265 & 1.843551 & -4.086336 \\
\hline $\mathrm{H}$ & -3.264874 & 1.055745 & -3.914986 \\
\hline $\mathrm{H}$ & -2.290243 & 1.892880 & -5.159044 \\
\hline $\mathrm{O}$ & -2.994526 & 3.101939 & -3.704049 \\
\hline $\mathrm{C}$ & -3.524530 & 3.090063 & -2.409814 \\
\hline $\mathrm{H}$ & -3.630849 & 4.138293 & -2.096613 \\
\hline $\mathrm{H}$ & -4.541319 & 2.647780 & -2.407595 \\
\hline 0 & -3.100670 & -0.876295 & 0.201719 \\
\hline B & -2.818646 & -1.008453 & -1.116805 \\
\hline $\mathrm{O}$ & -3.837304 & -1.356838 & -1.918414 \\
\hline $\mathrm{C}$ & -5.039182 & -1.289986 & -1.080185 \\
\hline C & -4.452883 & -1.418520 & 0.364664 \\
\hline C & -4.275524 & -2.853830 & 0.828608 \\
\hline $\mathrm{H}$ & -3.729438 & -3.457063 & 0.089490 \\
\hline $\mathrm{H}$ & -5.241843 & -3.333455 & 1.025560 \\
\hline $\mathrm{H}$ & -3.696259 & -2.861640 & 1.761263 \\
\hline C & -5.159246 & -0.594619 & 1.417764 \\
\hline $\mathrm{H}$ & -5.133380 & 0.475662 & 1.182573 \\
\hline $\mathrm{H}$ & -4.672688 & -0.743199 & 2.391593 \\
\hline $\mathrm{H}$ & -6.207086 & -0.906868 & 1.519741 \\
\hline $\mathrm{C}$ & -5.968833 & -2.408835 & -1.488918 \\
\hline $\mathrm{H}$ & -5.474229 & -3.384975 & -1.456401 \\
\hline $\mathrm{H}$ & -6.323014 & -2.241384 & -2.512620 \\
\hline $\mathrm{H}$ & -6.846914 & -2.437764 & -0.829661 \\
\hline C & -5.669195 & 0.062270 & -1.356898 \\
\hline $\mathrm{H}$ & -5.001216 & 0.882172 & -1.059518 \\
\hline $\mathrm{H}$ & -6.622379 & 0.178648 & -0.827056 \\
\hline $\mathrm{H}$ & -5.862981 & 0.153268 & -2.432798 \\
\hline $\mathrm{H}$ & -1.692336 & -0.927202 & -1.600957 \\
\hline $\mathrm{H}$ & 5.087466 & 0.636266 & 0.541395 \\
\hline
\end{tabular}

SCF energy: -3329.877514510

M06-D3 (Gas Phase) /6-31G(d)+SDD (Pd, Fe) opt. No imaginary frequency 


\begin{tabular}{|c|c|c|c|}
\hline \multicolumn{4}{|c|}{ Int-5RTania_conf 6} \\
\hline $\mathrm{P}$ & 0.296551 & -1.514851 & 0.594910 \\
\hline $\mathrm{P}$ & 0.361824 & 2. 261951 & 0.470039 \\
\hline $\mathrm{C}$ & -0.519444 & -3.106091 & 0.156426 \\
\hline C & -1.787478 & -5.507765 & -0.536264 \\
\hline C & -0.941914 & -3.338889 & -1.157408 \\
\hline $\mathrm{C}$ & -0.738918 & -4.097324 & 1.120109 \\
\hline $\mathrm{C}$ & -1.363719 & -5.291237 & 0.772044 \\
\hline $\mathrm{C}$ & -1.575385 & -4.528090 & -1.502858 \\
\hline $\mathrm{H}$ & -0.785805 & -2.585711 & -1.928144 \\
\hline $\mathrm{H}$ & -0.433464 & -3.938175 & 2.153618 \\
\hline $\mathrm{H}$ & -1.528628 & -6.051879 & 1.532457 \\
\hline $\mathrm{H}$ & -1.911995 & -4.681981 & -2.526319 \\
\hline $\mathrm{H}$ & -2.285399 & -6.438029 & -0.801908 \\
\hline $\mathrm{C}$ & 1.437801 & 3.494491 & -0.345515 \\
\hline C & 3.139887 & 5.367891 & -1.531074 \\
\hline C & 1.864756 & 4.618219 & 0.376011 \\
\hline C & 1.885046 & 3.312487 & -1.655455 \\
\hline C & 2.734523 & 4.244936 & -2.247682 \\
\hline $\mathrm{C}$ & 2.705904 & 5.552761 & -0.218695 \\
\hline $\mathrm{H}$ & 1.558422 & 2.437282 & -2.214877 \\
\hline $\mathrm{H}$ & 3.063894 & 4.103560 & -3.275858 \\
\hline $\mathrm{H}$ & 3.021450 & 6.430047 & 0.342301 \\
\hline $\mathrm{H}$ & 3.791185 & 6.104843 & -1.996405 \\
\hline $\mathrm{C}$ & -1.036257 & 3.197613 & 1.165741 \\
\hline $\mathrm{C}$ & -3.372991 & 4.465286 & 2.026650 \\
\hline C & -1.333225 & 4.494280 & 0.736209 \\
\hline C & -1.938388 & 2.527740 & 2.005395 \\
\hline C & -3.091539 & 3.165186 & 2.443559 \\
\hline C & -2.497746 & 5.123494 & 1.169550 \\
\hline $\mathrm{H}$ & -0.669278 & 5.016452 & 0.048895 \\
\hline $\mathrm{H}$ & -1.740305 & 1.501345 & 2.316999 \\
\hline $\mathrm{H}$ & -3.776825 & 2.643278 & 3.108588 \\
\hline $\mathrm{H}$ & -2.718986 & 6.133772 & 0.831723 \\
\hline $\mathrm{H}$ & -4.279569 & 4.961885 & 2.365749 \\
\hline $\mathrm{C}$ & 1.399517 & 1.724243 & 1.909535 \\
\hline C & 2.826306 & 0.805704 & 4.156238 \\
\hline C & 1.027672 & 2.094520 & 3.206316 \\
\hline C & 2.528436 & 0.895373 & 1.749588 \\
\hline $\mathrm{C}$ & 3.209217 & 0.429854 & 2.875526 \\
\hline $\mathrm{C}$ & 1.736792 & 1.652300 & 4.317841 \\
\hline $\mathrm{H}$ & 0.164846 & 2.739551 & 3.357481 \\
\hline $\mathrm{H}$ & 4.051887 & -0.254523 & 2.748762 \\
\hline $\mathrm{H}$ & 1.423111 & 1.960493 & 5.312753 \\
\hline $\mathrm{H}$ & 3.370272 & 0.432568 & 5.021203 \\
\hline $\mathrm{C}$ & 0.110464 & -1.484223 & 2.413337 \\
\hline C & -0.398812 & -1.572980 & 5.158539 \\
\hline $\mathrm{C}$ & -1.099616 & -1.001101 & 2.924463 \\
\hline C & 1.059526 & -2.019393 & 3.286814 \\
\hline C & 0.802956 & -2.062307 & 4.654932 \\
\hline C & -1.352081 & -1.043784 & 4.291145 \\
\hline $\mathrm{H}$ & -1.858460 & -0.624501 & 2.235656 \\
\hline $\mathrm{H}$ & 1.996548 & -2.422073 & 2.898890 \\
\hline $\mathrm{H}$ & 1.542664 & -2.489857 & 5.329699 \\
\hline $\mathrm{H}$ & -2.299991 & -0.672599 & 4.679117 \\
\hline $\mathrm{H}$ & -0.597875 & -1.615390 & 6.227500 \\
\hline $\mathrm{H}$ & 1.535197 & 4.766695 & 1.405348 \\
\hline $\mathrm{H}$ & 1.975783 & -4.122855 & 0.120754 \\
\hline C & 2.575907 & -3.218167 & 0.141222 \\
\hline C & 2.057208 & -1.894960 & 0.316894 \\
\hline $\mathrm{H}$ & 4.636116 & -3.967686 & -0.294404 \\
\hline C & 3.169792 & -0.977888 & 0.193105 \\
\hline C & 4.347518 & -1.767986 & -0.036444 \\
\hline $\mathrm{H}$ & 5.363089 & -1.419800 & -0.212110 \\
\hline C & 3.972167 & -3.137637 & -0.078301 \\
\hline $\mathrm{Fe}$ & 2.953589 & -2.057181 & -1.494618 \\
\hline $\mathrm{H}$ & 0.590289 & -1.173829 & -2.759654 \\
\hline C & 1.579026 & -1.591514 & -2.941053 \\
\hline C & 1.927180 & -2.972098 & -3.003663 \\
\hline $\mathrm{H}$ & 2.819788 & 0.255419 & -3.136515 \\
\hline $\mathrm{H}$ & 1.254185 & -3.809892 & -2.850015 \\
\hline C & 3.328562 & -3.059755 & -3.235611 \\
\hline $\mathrm{H}$ & 3.907664 & -3.974873 & -3.296536 \\
\hline
\end{tabular}

\begin{tabular}{|c|c|c|c|}
\hline $\mathrm{C}$ & 3.848393 & -1.737336 & -3.310769 \\
\hline $\mathrm{H}$ & 4.891169 & -1.477269 & -3.466557 \\
\hline $\mathrm{C}$ & 2.763842 & -0.830254 & -3.128742 \\
\hline C & 3.077740 & 0.514834 & 0.395894 \\
\hline $\mathrm{H}$ & 2.483020 & 0.971180 & -0.406484 \\
\hline $\mathrm{N}$ & 4.485338 & 1.136185 & 0.216563 \\
\hline $\mathrm{C}$ & 4.668272 & 2.503949 & 0.801363 \\
\hline $\mathrm{H}$ & 4.492221 & 2.477489 & 1.877512 \\
\hline $\mathrm{H}$ & 3.962475 & 3.186191 & 0.321216 \\
\hline $\mathrm{H}$ & 5.694615 & 2.818450 & 0.593757 \\
\hline $\mathrm{C}$ & 4.906678 & 1.162608 & -1.220163 \\
\hline $\mathrm{H}$ & 4.694976 & 0.193175 & -1.676163 \\
\hline $\mathrm{H}$ & 5.974086 & 1.394917 & -1.270416 \\
\hline $\mathrm{H}$ & 4.332013 & 1.953319 & -1.714408 \\
\hline $\mathrm{Pd}$ & -0.581198 & 0.458360 & -0.655345 \\
\hline C & -1.458555 & 1.874010 & -1.856378 \\
\hline $\mathrm{C}$ & -0.942172 & 2.135700 & -3.054668 \\
\hline $\mathrm{H}$ & 0.048906 & 1.803679 & -3.369323 \\
\hline $\mathrm{C}$ & -2.837841 & 2.319424 & -1.489258 \\
\hline $\mathrm{H}$ & -3.543450 & 1.486568 & -1.649207 \\
\hline $\mathrm{H}$ & -2.928945 & 2.611379 & -0.433983 \\
\hline C & -3.229830 & 3.479813 & -2.401906 \\
\hline $\mathrm{H}$ & -2.614182 & 4.368615 & -2.162228 \\
\hline $\mathrm{H}$ & -4.284838 & 3.745425 & -2.272475 \\
\hline O & -3.062347 & 3.106644 & -3.743979 \\
\hline $\mathrm{C}$ & -1.720313 & 2.923956 & -4.085180 \\
\hline $\mathrm{H}$ & -1.705952 & 2.408825 & -5.054082 \\
\hline $\mathrm{H}$ & -1.215822 & 3.902038 & -4.226904 \\
\hline $\mathrm{O}$ & -3.172109 & -0.683316 & 0.330042 \\
\hline B & -2.925783 & -0.949293 & -0.975548 \\
\hline $\mathrm{O}$ & -3.909104 & -1.550996 & -1.660599 \\
\hline C & -5.072860 & -1.564963 & -0.770876 \\
\hline $\mathrm{C}$ & -4.425169 & -1.379719 & 0.643648 \\
\hline C & -4.038679 & -2.689950 & 1.306051 \\
\hline $\mathrm{H}$ & -3.448898 & -3.328086 & 0.635136 \\
\hline $\mathrm{H}$ & -4.926767 & -3.249201 & 1.624294 \\
\hline $\mathrm{H}$ & -3.429690 & -2.487222 & 2.197288 \\
\hline C & -5.206072 & -0.507512 & 1.600841 \\
\hline $\mathrm{H}$ & -5.340626 & 0.507994 & 1.211140 \\
\hline $\mathrm{H}$ & -4.668287 & -0.438939 & 2.556722 \\
\hline $\mathrm{H}$ & -6.194368 & -0.939926 & 1.805781 \\
\hline $\mathrm{C}$ & -5.799056 & -2.876615 & -0.969524 \\
\hline $\mathrm{H}$ & -5.133445 & -3.737007 & -0.840481 \\
\hline $\mathrm{H}$ & -6.213165 & -2.917775 & -1.983518 \\
\hline $\mathrm{H}$ & -6.633694 & -2.966619 & -0.261286 \\
\hline $\mathrm{C}$ & -5.951938 & -0.403238 & -1.197325 \\
\hline $\mathrm{H}$ & -5.456625 & 0.565708 & -1.051325 \\
\hline $\mathrm{H}$ & -6.896084 & -0.392678 & -0.638998 \\
\hline $\mathrm{H}$ & -6.186979 & -0.505148 & -2.262881 \\
\hline $\mathrm{H}$ & -1.879363 & -0.672892 & -1.550101 \\
\hline $\mathrm{H}$ & 5.123504 & 0.507400 & 0.719483 \\
\hline
\end{tabular}

SCF energy: -3329.872119010

M06-D3 (Gas Phase) /6-31G(d)+SDD (Pd, Fe) opt. No imaginary frequency 


\begin{tabular}{|c|c|c|c|}
\hline \multicolumn{4}{|c|}{ Int-5RTania_conf7 } \\
\hline P & 0.468269 & 2.255039 & 0.255186 \\
\hline $\mathrm{C}$ & -0.882114 & -3.075414 & 0.375746 \\
\hline C & -2.518328 & -5.298202 & -0.127873 \\
\hline $\mathrm{C}$ & -1.205946 & -3.445566 & -0.934865 \\
\hline $\mathrm{C}$ & -1.407183 & -3.824817 & 1.432592 \\
\hline C & -2.217894 & -4.929303 & 1.178730 \\
\hline C & -2.009610 & -4.550660 & -1.188229 \\
\hline $\mathrm{H}$ & -0.834131 & -2.862770 & -1.774326 \\
\hline $\mathrm{H}$ & -1.201988 & -3.547790 & 2.465068 \\
\hline $\mathrm{H}$ & -2.615783 & -5.503046 & 2.013639 \\
\hline $\mathrm{H}$ & -2.247890 & -4.821661 & -2.215327 \\
\hline $\mathrm{H}$ & -3.151737 & -6.161411 & -0.321651 \\
\hline $\mathrm{C}$ & 1.717719 & 3.196127 & -0.705312 \\
\hline C & 3.651072 & 4.652699 & -2.112407 \\
\hline C & 2.693589 & 3.935353 & -0.013642 \\
\hline C & 1.710560 & 3.214142 & -2.102167 \\
\hline C & 2.672547 & 3.942373 & -2.800944 \\
\hline C & 3.658358 & 4.653290 & -0.717016 \\
\hline $\mathrm{H}$ & 0.932658 & 2.674603 & -2.638796 \\
\hline $\mathrm{H}$ & 2.651538 & 3.957417 & -3.888981 \\
\hline $\mathrm{H}$ & 4.402651 & 5.233319 & -0.174304 \\
\hline $\mathrm{H}$ & 4.399216 & 5.222440 & -2.659743 \\
\hline C & -0.703011 & 3.500097 & 0.864090 \\
\hline C & -2.681426 & 5.304035 & 1.644589 \\
\hline C & -0.683572 & 4.811666 & 0.383196 \\
\hline C & -1.733914 & 3.089939 & 1.721958 \\
\hline C & -2.708512 & 3.993960 & 2.121824 \\
\hline C & -1.675328 & 5.708423 & 0.773897 \\
\hline $\mathrm{H}$ & 0.095485 & 5.139441 & -0.303961 \\
\hline $\mathrm{H}$ & -1.777658 & 2.054988 & 2.069865 \\
\hline $\mathrm{H}$ & -3.500266 & 3.674403 & 2.796901 \\
\hline $\mathrm{H}$ & -1.657290 & 6.728290 & 0.395871 \\
\hline $\mathrm{H}$ & -3.452015 & 6.008817 & 1.949435 \\
\hline $\mathrm{C}$ & 1.452874 & 1.707309 & 1.714928 \\
\hline C & 2.741648 & 0.683249 & 3.987822 \\
\hline $\mathrm{C}$ & 1.145382 & 2.185129 & 2.990277 \\
\hline $\mathrm{C}$ & 2.456785 & 0.725010 & 1.579212 \\
\hline C & 3.062179 & 0.203971 & 2.722352 \\
\hline $\mathrm{C}$ & 1.794553 & 1.689498 & 4.117850 \\
\hline $\mathrm{H}$ & 0.382543 & 2.951205 & 3.113537 \\
\hline $\mathrm{H}$ & 3.783779 & -0.608399 & 2.620978 \\
\hline $\mathrm{H}$ & 1.537648 & 2.078497 & 5.100712 \\
\hline $\mathrm{H}$ & 3.223676 & 0.261604 & 4.867034 \\
\hline $\mathrm{C}$ & 0.071270 & -1.437235 & 2.499943 \\
\hline $\mathrm{C}$ & -0.277485 & -1.407073 & 5.270769 \\
\hline $\mathrm{C}$ & -0.949273 & -0.675266 & 3.075832 \\
\hline $\mathrm{C}$ & 0.916936 & -2.185939 & 3.323500 \\
\hline $\mathrm{C}$ & 0.741453 & -2.169343 & 4.703674 \\
\hline $\mathrm{C}$ & -1.123330 & -0.659106 & 4.455705 \\
\hline $\mathrm{H}$ & -1.637777 & -0.133606 & 2.425518 \\
\hline $\mathrm{H}$ & 1.704531 & -2.800987 & 2.885069 \\
\hline $\mathrm{H}$ & 1.394961 & -2.764332 & 5.339081 \\
\hline $\mathrm{H}$ & -1.928034 & -0.069924 & 4.894255 \\
\hline $\mathrm{H}$ & -0.418099 & -1.405075 & 6.349872 \\
\hline $\mathrm{H}$ & 2.686879 & 3.964184 & 1.077823 \\
\hline $\mathrm{H}$ & 1.592054 & -4.315993 & 0.204264 \\
\hline $\mathrm{C}$ & 2.247745 & -3.451653 & 0.152869 \\
\hline $\mathrm{C}$ & 1.822009 & -2.092686 & 0.293011 \\
\hline $\mathrm{H}$ & 4.236975 & -4.345043 & -0.341035 \\
\hline $\mathrm{C}$ & 2.987250 & -1.253847 & 0.086565 \\
\hline $\mathrm{C}$ & 4.098297 & -2.121671 & -0.165654 \\
\hline $\mathrm{H}$ & 5.119896 & -1.840515 & -0.402097 \\
\hline C & 3.635715 & -3.466612 & -0.132158 \\
\hline $\mathrm{Fe}$ & 2.628177 & -2.391629 & -1.550009 \\
\hline $\mathrm{H}$ & 0.251968 & -1.534183 & -2.828271 \\
\hline $\mathrm{C}$ & 1.232656 & -1.973583 & -2.995866 \\
\hline $\mathrm{C}$ & 1.564624 & -3.359381 & -3.001821 \\
\hline $\mathrm{H}$ & 2.482094 & -0.152487 & -3.297895 \\
\hline $\mathrm{H}$ & 0.887616 & -4.184801 & -2.805825 \\
\hline C & 2.961002 & -3.472202 & -3.251006 \\
\hline $\mathrm{H}$ & 3.529048 & -4.395866 & -3.279259 \\
\hline $\mathrm{C}$ & 3.493947 & -2.160194 & -3.391485 \\
\hline
\end{tabular}

\begin{tabular}{|c|c|c|c|}
\hline $\mathrm{H}$ & 4.536756 & -1.919633 & -3.575040 \\
\hline $\mathrm{C}$ & 2.421732 & -1.235146 & -3.233766 \\
\hline $\mathrm{C}$ & 2.896241 & 0.240219 & 0.215574 \\
\hline $\mathrm{H}$ & 2.170612 & 0.582135 & -0.537867 \\
\hline $\mathrm{N}$ & 4.167655 & 1.025280 & -0.184743 \\
\hline $\mathrm{C}$ & 4.533925 & 0.877861 & -1.624874 \\
\hline $\mathrm{H}$ & 3.686381 & 1.213786 & -2.227514 \\
\hline $\mathrm{H}$ & 4.760292 & -0.168439 & -1.839097 \\
\hline $\mathrm{H}$ & 5.399858 & 1.514169 & -1.827721 \\
\hline $\mathrm{C}$ & 5.363705 & 0.869581 & 0.698072 \\
\hline $\mathrm{H}$ & 5.098552 & 1.151302 & 1.718151 \\
\hline $\mathrm{H}$ & 6.145901 & 1.535397 & 0.323832 \\
\hline $\mathrm{H}$ & 5.712457 & -0.164065 & 0.671686 \\
\hline $\mathrm{Pd}$ & -0.706345 & 0.434487 & -0.641884 \\
\hline $\mathrm{H}$ & 3.864522 & 2.009152 & -0.081205 \\
\hline $\mathrm{P}$ & 0.118760 & -1.557941 & 0.676881 \\
\hline $\mathrm{C}$ & -1.544850 & 1.755368 & -1.976383 \\
\hline C & -1.117496 & 1.649557 & -3.232871 \\
\hline $\mathrm{H}$ & -0.241163 & 1.059472 & -3.518355 \\
\hline $\mathrm{C}$ & -2.757439 & 2.552087 & -1.624112 \\
\hline $\mathrm{H}$ & -3.628785 & 1.879435 & -1.547415 \\
\hline $\mathrm{H}$ & -2.663790 & 3.071171 & -0.661103 \\
\hline C & -2.991612 & 3.570734 & -2.739944 \\
\hline $\mathrm{H}$ & -2.178405 & 4.324198 & -2.726234 \\
\hline $\mathrm{H}$ & -3.946647 & 4.090165 & -2.607392 \\
\hline $\mathrm{O}$ & -3.048060 & 2.920376 & -3.981265 \\
\hline $\mathrm{C}$ & -1.822289 & 2.372012 & -4.361027 \\
\hline $\mathrm{H}$ & -2.024125 & 1.684934 & -5.192516 \\
\hline $\mathrm{H}$ & -1.142871 & 3.161194 & -4.746646 \\
\hline O & -3.040422 & -0.918558 & 0.622389 \\
\hline B & -3.035139 & -0.762424 & -0.723047 \\
\hline O & -4.236124 & -0.668983 & -1.316265 \\
\hline $\mathrm{C}$ & -5.205521 & -1.079926 & -0.294713 \\
\hline C & -4.436360 & -0.790296 & 1.036280 \\
\hline C & -4.682722 & -1.782467 & 2.151370 \\
\hline $\mathrm{H}$ & -4.411813 & -2.801671 & 1.854501 \\
\hline $\mathrm{H}$ & -5.737393 & -1.771031 & 2.457070 \\
\hline $\mathrm{H}$ & -4.072925 & -1.516123 & 3.024990 \\
\hline C & -4.598794 & 0.634477 & 1.538482 \\
\hline $\mathrm{H}$ & -4.372526 & 1.371117 & 0.754394 \\
\hline $\mathrm{H}$ & -3.904712 & 0.799201 & 2.375482 \\
\hline $\mathrm{H}$ & -5.615425 & 0.819542 & 1.905789 \\
\hline $\mathrm{C}$ & -5.457605 & -2.558027 & -0.531290 \\
\hline $\mathrm{H}$ & -4.540748 & -3.151125 & -0.401742 \\
\hline $\mathrm{H}$ & -5.810036 & -2.698596 & -1.559539 \\
\hline $\mathrm{H}$ & -6.224155 & -2.947897 & 0.149390 \\
\hline $\mathrm{C}$ & -6.471190 & -0.277477 & -0.486995 \\
\hline $\mathrm{H}$ & -6.278937 & 0.801093 & -0.487653 \\
\hline $\mathrm{H}$ & -7.194089 & -0.505280 & 0.308030 \\
\hline $\mathrm{H}$ & -6.933082 & -0.538199 & -1.446019 \\
\hline $\mathrm{H}$ & -2.009012 & -0.772642 & -1.399740 \\
\hline
\end{tabular}

SCF energy: -3329.871454530

M06-D3 (Gas Phase)/6-31G(d)+SDD (Pd, Fe) opt. No imaginary frequency 


\begin{tabular}{|c|c|c|c|}
\hline \multicolumn{4}{|c|}{ Int-5STania_conf3 } \\
\hline $\mathrm{P}$ & 0.159360 & 2.178810 & 0.412471 \\
\hline $\mathrm{C}$ & -0.434981 & -3.248592 & 0.481071 \\
\hline $\mathrm{C}$ & -1.658696 & -5.743365 & 0.086436 \\
\hline $\mathrm{C}$ & -0.865581 & -3.643704 & -0.791118 \\
\hline C & -0.628516 & -4.126401 & 1.554432 \\
\hline $\mathrm{C}$ & -1.232850 & -5.364694 & 1.354984 \\
\hline $\mathrm{C}$ & -1.471820 & -4.879191 & -0.989656 \\
\hline $\mathrm{H}$ & -0.730413 & -2.986824 & -1.647419 \\
\hline $\mathrm{H}$ & -0.314821 & -3.847119 & 2.559019 \\
\hline $\mathrm{H}$ & -1.374825 & -6.033430 & 2.201485 \\
\hline $\mathrm{H}$ & -1.802601 & -5.163370 & -1.986971 \\
\hline $\mathrm{H}$ & -2.136913 & -6.708860 & -0.064616 \\
\hline $\mathrm{C}$ & 1.208221 & 3.354246 & -0.527101 \\
\hline $\mathrm{C}$ & 2.879499 & 5.096920 & -1.945897 \\
\hline $\mathrm{C}$ & 2.104849 & 4.194385 & 0.157193 \\
\hline $\mathrm{C}$ & 1.152358 & 3.410982 & -1.922606 \\
\hline $\mathrm{C}$ & 1.981158 & 4.280833 & -2.627704 \\
\hline $\mathrm{C}$ & 2.938775 & 5.056277 & -0.552533 \\
\hline $\mathrm{H}$ & 0.446851 & 2.774345 & -2.452553 \\
\hline $\mathrm{H}$ & 1.921841 & 4.320942 & -3.713812 \\
\hline $\mathrm{H}$ & 3.622148 & 5.711115 & -0.014946 \\
\hline $\mathrm{H}$ & 3.526395 & 5.776173 & -2.497294 \\
\hline C & -1.210180 & 3.127549 & 1.125714 \\
\hline $\mathrm{C}$ & -3.419226 & 4.483965 & 2.143492 \\
\hline $\mathrm{C}$ & -1.284610 & 4.518177 & 1.019342 \\
\hline $\mathrm{C}$ & -2.252200 & 2.414541 & 1.735963 \\
\hline C & -3.347072 & 3.095631 & 2.251299 \\
\hline $\mathrm{C}$ & -2.391686 & 5.191829 & 1.527961 \\
\hline $\mathrm{H}$ & -0.491346 & 5.080536 & 0.528512 \\
\hline $\mathrm{H}$ & -2.214438 & 1.324490 & 1.787717 \\
\hline $\mathrm{H}$ & -4.150960 & 2.541643 & 2.733252 \\
\hline $\mathrm{H}$ & -2.451313 & 6.274356 & 1.439148 \\
\hline $\mathrm{H}$ & -4.282968 & 5.015034 & 2.537863 \\
\hline $\mathrm{C}$ & 1.262600 & 1.751175 & 1.820645 \\
\hline $\mathrm{C}$ & 2.866483 & 1.029273 & 4.001126 \\
\hline $\mathrm{C}$ & 0.915283 & 2.130323 & 3.119392 \\
\hline C & 2.435434 & 0.997072 & 1.615228 \\
\hline $\mathrm{C}$ & 3.209737 & 0.627198 & 2.715546 \\
\hline $\mathrm{C}$ & 1.717835 & 1.783871 & 4.201887 \\
\hline $\mathrm{H}$ & 0.005723 & 2.702956 & 3.292107 \\
\hline $\mathrm{H}$ & 4.081078 & -0.012661 & 2.567392 \\
\hline $\mathrm{H}$ & 1.430627 & 2.090652 & 5.205248 \\
\hline $\mathrm{H}$ & 3.485207 & 0.733341 & 4.845624 \\
\hline $\mathrm{C}$ & 0.183640 & -1.418590 & 2.557732 \\
\hline $\mathrm{C}$ & -0.284447 & -1.317391 & 5.307951 \\
\hline C & -1.056285 & -0.991130 & 3.048251 \\
\hline $\mathrm{C}$ & 1.180314 & -1.809192 & 3.453416 \\
\hline $\mathrm{C}$ & 0.943870 & -1.758147 & 4.825101 \\
\hline $\mathrm{C}$ & -1.286283 & -0.935987 & 4.417432 \\
\hline $\mathrm{H}$ & -1.854375 & -0.746119 & 2.344906 \\
\hline $\mathrm{H}$ & 2.139671 & -2.172793 & 3.082191 \\
\hline $\mathrm{H}$ & 1.721320 & -2.073701 & 5.518956 \\
\hline $\mathrm{H}$ & -2.254666 & -0.607696 & 4.792083 \\
\hline $\mathrm{H}$ & -0.467094 & -1.284975 & 6.380235 \\
\hline $\mathrm{H}$ & 2.146241 & 4.178289 & 1.247997 \\
\hline $\mathrm{H}$ & 2.221032 & -4.134674 & 0.551437 \\
\hline $\mathrm{C}$ & 2.740127 & -3.189130 & 0.423261 \\
\hline C & 2.115679 & -1.901584 & 0.441238 \\
\hline $\mathrm{H}$ & 4.842092 & -3.817063 & -0.007328 \\
\hline $\mathrm{C}$ & 3.146780 & -0.920432 & 0.153726 \\
\hline C & 4.375012 & -1.631530 & -0.026315 \\
\hline $\mathrm{H}$ & 5.343520 & -1.219860 & -0.294581 \\
\hline C & 4.116803 & -3.022018 & 0.129438 \\
\hline $\mathrm{Fe}$ & 2.962832 & -2.246414 & -1.369118 \\
\hline $\mathrm{H}$ & 0.510625 & -1.800176 & -2.707519 \\
\hline $\mathrm{C}$ & 1.530117 & -2.153589 & -2.836307 \\
\hline
\end{tabular}

\begin{tabular}{|c|c|c|c|}
\hline $\mathrm{C}$ & 2.004747 & -3.487218 & -2.675585 \\
\hline $\mathrm{H}$ & 2.577881 & -0.268749 & -3.392155 \\
\hline $\mathrm{H}$ & 1.415575 & -4.345020 & -2.366395 \\
\hline C & 3.405135 & -3.483989 & -2.931017 \\
\hline $\mathrm{H}$ & 4.067411 & -4.339541 & -2.852440 \\
\hline C & 3.797443 & -2.151044 & -3.239181 \\
\hline $\mathrm{H}$ & 4.808539 & -1.824885 & -3.463158 \\
\hline C & 2.633834 & -1.331846 & -3.183104 \\
\hline C & 2.837841 & 0.545867 & 0.227653 \\
\hline $\mathrm{H}$ & 1.998555 & 0.722177 & -0.463527 \\
\hline $\mathrm{N}$ & 3.909823 & 1.507996 & -0.325166 \\
\hline C & 4.191736 & 1.309080 & -1.775798 \\
\hline $\mathrm{H}$ & 3.248374 & 1.401914 & -2.320349 \\
\hline $\mathrm{H}$ & 4.620296 & 0.316349 & -1.931164 \\
\hline $\mathrm{H}$ & 4.884113 & 2.089469 & -2.104006 \\
\hline C & 5.172711 & 1.646382 & 0.459998 \\
\hline $\mathrm{H}$ & 4.930034 & 1.936712 & 1.483732 \\
\hline $\mathrm{H}$ & 5.775733 & 2.427527 & -0.011483 \\
\hline $\mathrm{H}$ & 5.719555 & 0.701633 & 0.454077 \\
\hline $\mathrm{Pd}$ & -0.650472 & 0.264903 & -0.623309 \\
\hline $\mathrm{H}$ & 3.438625 & 2.426636 & -0.255480 \\
\hline $\mathrm{P}$ & 0.342176 & -1.598000 & 0.745864 \\
\hline $\mathrm{C}$ & -1.566665 & 1.484889 & -1.996898 \\
\hline $\mathrm{C}$ & -2.502694 & 2.406683 & -1.787620 \\
\hline $\mathrm{H}$ & -2.762003 & 2.780932 & -0.795884 \\
\hline $\mathrm{C}$ & -1.231021 & 0.999723 & -3.380608 \\
\hline $\mathrm{H}$ & -0.144197 & 0.872250 & -3.529292 \\
\hline $\mathrm{H}$ & -1.686170 & 0.011451 & -3.556876 \\
\hline $\mathrm{C}$ & -1.781004 & 2.000289 & -4.403694 \\
\hline $\mathrm{H}$ & -1.736385 & 1.585947 & -5.416780 \\
\hline $\mathrm{H}$ & -1.181224 & 2.932365 & -4.384859 \\
\hline 0 & -3.124447 & 2.278506 & -4.128179 \\
\hline $\mathrm{C}$ & -3.278137 & 2.998340 & -2.938455 \\
\hline $\mathrm{H}$ & -2.967587 & 4.054028 & -3.077507 \\
\hline $\mathrm{H}$ & -4.351497 & 3.007444 & -2.708468 \\
\hline 0 & -3.081202 & -0.918218 & 0.373392 \\
\hline B & -2.821091 & -1.044383 & -0.951214 \\
\hline 0 & -3.873431 & -1.251944 & -1.755404 \\
\hline $\mathrm{C}$ & -5.052784 & -1.055280 & -0.904413 \\
\hline $\mathrm{C}$ & -4.480772 & -1.334064 & 0.521183 \\
\hline $\mathrm{C}$ & -4.433948 & -2.806463 & 0.890948 \\
\hline $\mathrm{H}$ & -3.972768 & -3.410736 & 0.097221 \\
\hline $\mathrm{H}$ & -5.436299 & -3.202838 & 1.093423 \\
\hline $\mathrm{H}$ & -3.826160 & -2.926364 & 1.798016 \\
\hline $\mathrm{C}$ & -5.103249 & -0.517980 & 1.630532 \\
\hline $\mathrm{H}$ & -5.001425 & 0.558336 & 1.449399 \\
\hline $\mathrm{H}$ & -4.619415 & -0.759360 & 2.586908 \\
\hline $\mathrm{H}$ & -6.171198 & -0.752603 & 1.731300 \\
\hline $\mathrm{C}$ & -6.134357 & -2.010052 & -1.350934 \\
\hline $\mathrm{H}$ & -5.782194 & -3.046388 & -1.371123 \\
\hline $\mathrm{H}$ & -6.471033 & -1.745430 & -2.359896 \\
\hline $\mathrm{H}$ & -7.001090 & -1.947179 & -0.679096 \\
\hline $\mathrm{C}$ & -5.481050 & 0.387121 & -1.111351 \\
\hline $\mathrm{H}$ & -4.713933 & 1.092272 & -0.757910 \\
\hline $\mathrm{H}$ & -6.422212 & 0.604032 & -0.591248 \\
\hline $\mathrm{H}$ & -5.632984 & 0.558391 & -2.184257 \\
\hline $\mathrm{H}$ & -1.688050 & -1.096055 & -1.436633 \\
\hline
\end{tabular}

SCF energy: -3329.876729790

M06-D3 (Gas Phase)/6-31G(d)+SDD (Pd, Fe) opt. No imaginary frequency 


\section{References}

[1] Gaussian 16, Revision A.03, Frisch, M. J.; Trucks, G. W.; Schlegel, H. B.; Scuseria, G. E.; Robb, M. A.; Cheeseman, J. R.; Scalmani, G.; Barone, V.; Mennucci, B.; Petersson, G. A.; Nakatsuji, H.; Caricato, M.; Li, X.; Hratchian, H. P.; Izmaylov, A. F.; Bloino, J.; Zheng, G.; Sonnenberg, J. L.; Hada, M.; Ehara, M.; Toyota, K.; Fukuda, R.; Hasegawa, J.; Ishida, M.; Nakajima, T.; Honda, Y.; Kitao, O.; Nakai, H.; Vreven, T.; Montgomery, Jr., J. A.; Peralta, J. E.; Ogliaro, F.; Bearpark, M.; Heyd, J. J.; Brothers, E.; Kudin, K. N.; Staroverov, V. N.; Kobayashi, R.; Normand, J.; Raghavachari, K.; Rendell, A.; Burant, J. C.; Iyengar, S. S.; Tomasi, J.; Cossi, M.; Rega, N.; Millam, J. M.; Klene, M.; Knox, J. E.; Cross, J. B.; Bakken, V.; Adamo, C.; Jaramillo, J.; Gomperts, R.; Stratmann, R. E.; Yazyev, O.; Austin, A. J.; Cammi, R.; Pomelli, C.; Ochterski, J. W.; Martin, R. L.; Morokuma, K.; Zakrzewski, V. G.; Voth, G. A.; Salvador, P.; Dannenberg, J. J.; Dapprich, S.; Daniels, A. D.; Farkas, Ö.; Foresman, J. B.; Ortiz, J. V.; Cioslowski, J.; Fox, D. J. Gaussian, Inc., Wallingford CT, 2016.

[2] Zhao, Y.; Truhlar, D. G. The M06 suite of density functionals for main group thermochemistry, thermochemical kinetics, noncovalent interactions, excited states, and transition elements: two new functionals and systematic testing of four M06-class functionals and 12 other functionals. Theor. Chem. Acc. 2008, 120, 215-241.

[3] a) Hehre, J. W.; Ditchfield, R.; Pople, J. A. Self-Consistent Molecular Orbital Methods. XII. Further Extensions of Gaussian-Type Basis Sets for Use in Molecular Orbital Studies of Organic Molecules. J. Chem. Phys. 1972, 56, 2257-2261; b) Hariharan, P. C.; Pople, J. A. The influence of polarization functions on molecular orbital hydrogenation energies. Theor. Chim. Acta. 1973, 28, 213-222.

[4] a) Hay, P. J.; Wadt, W. R. Ab initio effective core potentials for molecular calculations potentials for main group elements Na to Bi. J. Chem. Phys. 1985, 82, 284-298; b) Check, C. E.; Faust, T. O.; Bailey, J. M.; Wright, B. J.; Gilbert, T. M.; Sunderlin, L. S. Addition of Polarization and Diffuse Functions to the LANL2DZ Basis Set for P-Block Elements. J. Phys. Chem. A 2001, $105,8111-8116$. 
[5] Grimme, S.; Antony, J.; Ehrlich, S.; Krieg, H.; A consistent and accurate ab initio parametrization of density functional dispersion correction (DFT-D) for the 94 elements H-Pu. $J$. Chem. Phys. 2010, 132, 154104.

[6] Dolg, M.; Wedig, U.; Stoll, H.; Preuß, H. Energy-adjusted ab initio pseudopotentials for the first row transition elements. J. Chem. Phys. 1987, 86, 866-872.

[7] Marenich, A. V.; Cramer, C. J.; Truhlar, D. G. Universal Solvation Model Based on Solute Electron Density and on a Continuum Model of the Solvent Defined by the Bulk Dielectric Constant and Atomic Surface Tensions. J. Phys. Chem. B 2009, 113, 6378-6396.

[8] Grimme, S. Supramolecular Binding Thermodynamics by Dispersion-Corrected Density Functional Theory. Chemistry - A European Journal 2012, 18 , 9955-9964. 